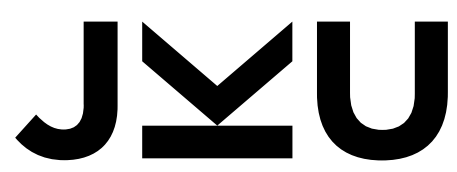

JOHANNES KEPLER

UNIVERSITÄT LINZ

Eingereicht von

Luca Benvenuti

Angefertigt am

Department of Particulate

Flow Modelling

Erstbeurteiler

Stefan Pirker

\title{
Identification of DEM
}

Zweitbeurteiler

Gerald G. Pereira

Simulation Parameters by

Mitbetreuung

Thomas Lichtenegger

März 2016

\section{Artificial Neural Networks and Bulk Experiments}

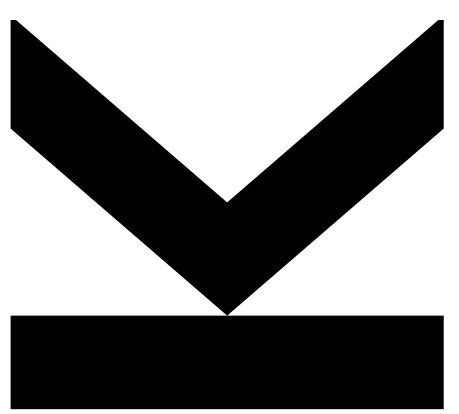

\section{Dissertation}

zur Erlangung des akademischen Grades

Doktor der Technischen Wissenschaften

im Doktoratsstudium der

Technischen Wissenschaften 



\section{Contents}

Abstract $\quad$ v

Zusammenfassung vi

Publications viii

$\begin{array}{ll}\text { List of Figures } & \text { ix }\end{array}$

List of Tables $\quad x i$

Acronyms xii

I Introduction 1

1 Prologue 2

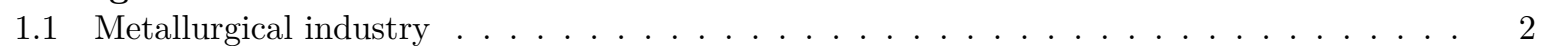

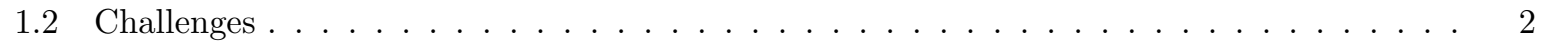

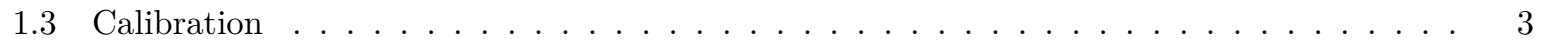

1.4 Neural Network Calibration . . . . . . . . . . . . . . . . . . . . . . . . . . . 4

1.5 Parameters Identification $\ldots \ldots \ldots \ldots \ldots \ldots \ldots \ldots$

2 Motivation and Insufficiency of the State of the Art $\quad 6$

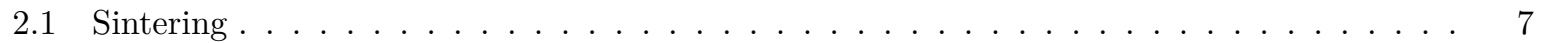

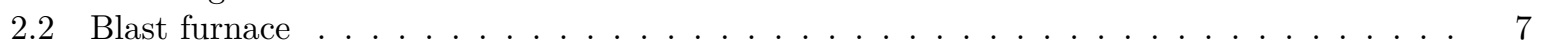

$2.2 .1 \quad$ Blast furnace structure $\ldots \ldots \ldots \ldots \ldots \ldots$

$2.2 .2 \quad$ Blast furnace chemistry $\ldots \ldots \ldots \ldots \ldots \ldots \ldots$

2.3 Materials characterization $\ldots \ldots \ldots \ldots \ldots \ldots \ldots \ldots$

2.4 DEM Simulations $\ldots \ldots \ldots \ldots \ldots$

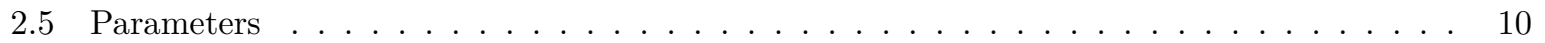

$2.5 .1 \quad$ Direct Determination $\ldots \ldots \ldots \ldots$

3 Aim of this Thesis 12

3.1 Aim for Neural Network . . . . . . . . . . . . . . . . . . . . . . . . . . 12

$3.1 .1 \quad$ Applicability and scope $\ldots \ldots \ldots \ldots \ldots$

\begin{tabular}{lll}
\hline II Theory & 16
\end{tabular}

$\begin{array}{lll}4 & \text { Discrete Element Method } & 17\end{array}$

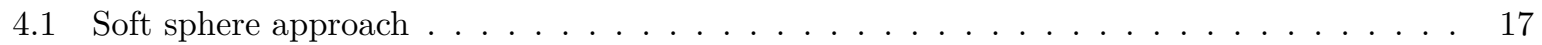

$4.1 .1 \quad$ Hertz $\ldots \ldots \ldots \ldots$ 
4.1 .2 Elasto-plastic-spring-dashpot 2 model . . . . . . . . . . . . . . . . . . . . . . . . 19

4.2 Literature Values $\ldots \ldots \ldots$. . . . . . . . . . . . . . . . . . . . . . . . . . . . . . . . 20

4.2 .1 Limitations . . . . . . . . . . . . . . . . . . . . . . . 20

4.3 Unresolved CFD-DEM . . . . . . . . . . . . . . . . . . . . . . . . . . . . . . . . . 20

4.3 .1 Governing Equations . . . . . . . . . . . . . . . . . . . . . 23

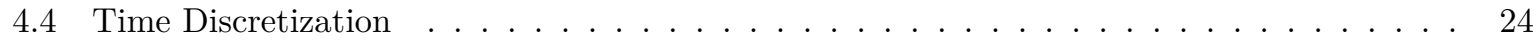

4.5 Coupled Simulations . . . . . . . . . . . . . . . . . . . . . . . . . . . . . . . . . . 24

$\begin{array}{llr}5 & \text { Artificial Neural Network } & \mathbf{2 6}\end{array}$

5.1 Probability theory . . . . . . . . . . . . . . . . . . . . . . . 26

5.1 .1 The Gaussian Distribution $\ldots \ldots \ldots$. . . . . . . . . . . . 26

5.2 Principal Component Analysis (PCA) . . . . . . . . . . . . . . . . . . . . . 27

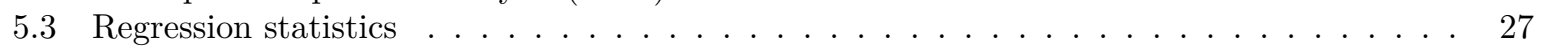

5.3 .1 Learning processes . . . . . . . . . . . . . . . . . . . . 27

5.3 .2 The Standard Bayesian Linear Model . . . . . . . . . . . . . . . . . . . . . . . . . 28

5.3 .3 Gaussian Process . . . . . . . . . . . . . . . . . . . . . . . . . 29

5.4 Introduction on Artificial Neural Networks . . . . . . . . . . . . . . . . . . . . . . . . . 30

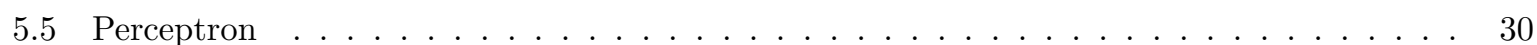

5.5 .1 Double Moon . . . . . . . . . . . . . . . . . . . . . . . 31

5.6 Multilayer . . . . . . . . . . . . . . . . . . . . . . . . . . . . . . 32

5.7 Batch Learning . . . . . . . . . . . . . . . . . . . . . . . . . . . . . . . . . . . . 33

5.8 Backpropagation algorithm . . . . . . . . . . . . . . . . . . . . . . . . . . 34

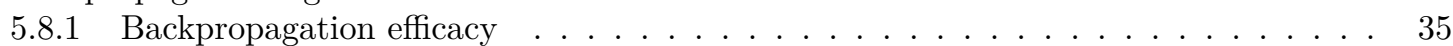

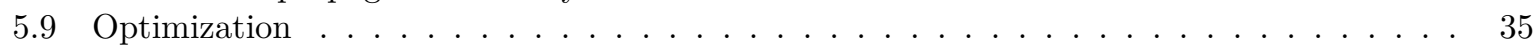

5.9 .1 Conjugate Gradient Algorithm . . . . . . . . . . . . . . . . . . . . . . . . . 35

5.10 Generalization . . . . . . . . . . . . . . . . . . . . . 36

5.11 Cross validation $\ldots \ldots \ldots \ldots \ldots \ldots$

5.12 ANNs training for DEM identification . . . . . . . . . . . . . . . . . . . . 37

5.13 Comparison of regression methods . . . . . . . . . . . . . . . . . . . . . . 38

III Identification of DEM parameters $\quad 39$

6 Experimental Characterization 40

6.1 Particle Distribution . . . . . . . . . . . . . . . . . . . . . . . . . . . 40

6.2 Drained angle of repose $(\mathrm{p}-\mathrm{p})-$ small scale . . . . . . . . . . . . . . . . . . . . . . . 42

6.3 Drained angle of repose $(\mathrm{p}-\mathrm{p})$ - large scale . . . . . . . . . . . . . . . . . . . . . . . . . . 42

6.4 Jenike Shear Cell tester . . . . . . . . . . . . . . . . . . . . . . . . . . . . . . . . 43

6.5 Schulze Ring Shear Cell tester $(\mathrm{p}-\mathrm{p})$. . . . . . . . . . . . . . . . . . . . . . . . . 43

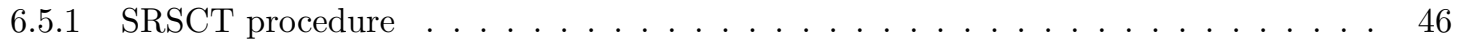

6.5 .2 SRSCT results . . . . . . . . . . . . . . . . . . . . . 47

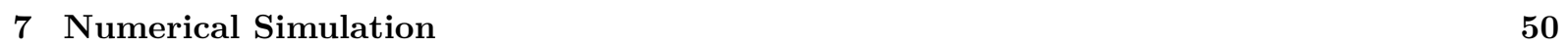

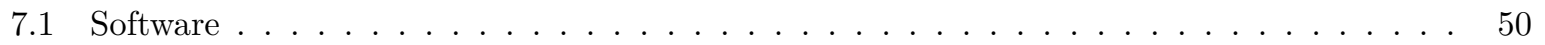

7.2 Microscopic parameters . . . . . . . . . . . . . . . . . . . . . . 50

7.3 Macroscopic parameters . . . . . . . . . . . . . . . . . . . . . . . . . . . 51

7.4 Drained Angle of Repose Simulation . . . . . . . . . . . . . . . . . . . . . . . . . 51

7.5 Shear Cell Simulation . . . . . . . . . . . . . . . . . . . . . . . . . 51

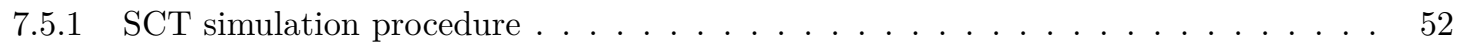

7.5 .2 SCT Simulation Input Parameters . . . . . . . . . . . . . . . . . . . . . . . . . . . . . . . . . . . . . . . .

7.5 .3 SCT Simulation Output Parameters . . . . . . . . . . . . . . . . . . . . . . . . . . . . . 52

7.6 Influence of variations of input parameters . . . . . . . . . . . . . . . . . . . . 53 
8 Artificial Neural Network Generalization

8.1 Principal Component Analysis (PCA) Results . . . . . . . . . . . . . . . . . . . 55

8.2 Regression statistic training concept $\ldots \ldots \ldots \ldots \ldots \ldots \ldots \ldots \ldots$

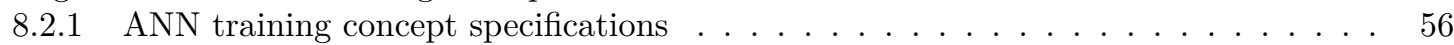

8.2 .2 Sinter fine ANN training . . . . . . . . . . . . . . . . . . . . . . 56

8.3 Statistical tools comparison $\ldots \ldots \ldots \ldots \ldots \ldots \ldots \ldots$

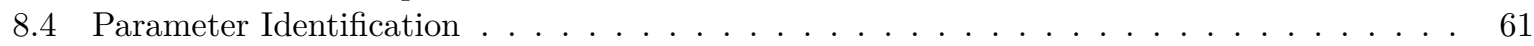

8.4 .1 Computational Condiderations . . . . . . . . . . . . . . . . . . . 61

$8.4 .2 \quad$ Decisional Limits . . . . . . . . . . . . . . . . . . . . . . . . . . . . . . . . 61

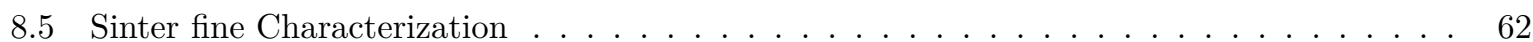

8.5 .1 Parameter space plot. . . . . . . . . . . . . . . . . . . . 63

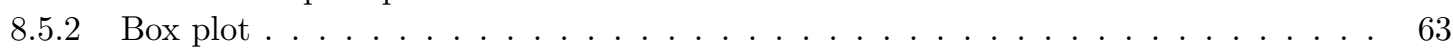

$8.5 .3 \quad$ Density plot . . . . . . . . . . . . . . . . . . . . . . . . . . . . 65

8.5 .4 Reliability Considerations . . . . . . . . . . . . . . . . . . . . . . 65

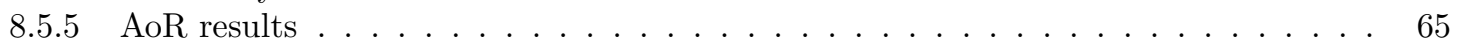

8.5 .6 Merge results . . . . . . . . . . . . . . . . . . . . . . . . 65

8.6 Remaining Materials Characterization $\ldots \ldots \ldots \ldots \ldots$

8.6 .1 Coke coarse Characterization . . . . . . . . . . . . . . . . . . . 69

8.6 .2 Coke fine Characterization . . . . . . . . . . . . . . . . . . . . 69

8.6 .3 Iron ore coarse Characterization . . . . . . . . . . . . . . . . . . . . . . 74

8.6 .4 Iron ore fine Characterization . . . . . . . . . . . . . . . . . . . . . . . . 74

8.6 .5 Limestone coarse Characterization . . . . . . . . . . . . . . . . . 74

8.6 .6 Limestone fine Characterization . . . . . . . . . . . . . . . . . . . . . 74

8.6 .7 Sinter coarse Characterization . . . . . . . . . . . . . . . . . . . . . . . 74

$8.7 \quad$ Discussion of Model Limitations $\ldots \ldots \ldots \ldots$. . . . . . . . . . . . . . . . 83

\begin{tabular}{lll}
\hline IV Applications & 84
\end{tabular}

\begin{tabular}{ll|l}
9 & Sinter Segregator & 85
\end{tabular}

$9.1 \quad$ Material properties for sinter chute simulation $\ldots \ldots \ldots \ldots \ldots \ldots \ldots$

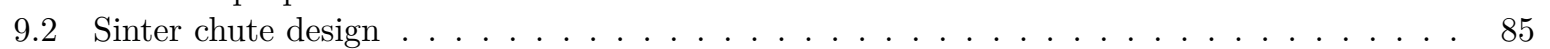

9.3 Numerical considerations for sinter chute simulation $\ldots \ldots \ldots \ldots \ldots \ldots \ldots$. . . . . . 85

9.4 Sinter chute simulation phases . . . . . . . . . . . . . . . . . . . . . . 85

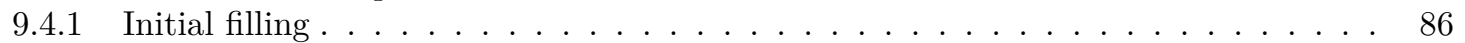

9.4 .2 Moving bottom box $\ldots \ldots \ldots \ldots \ldots \ldots$

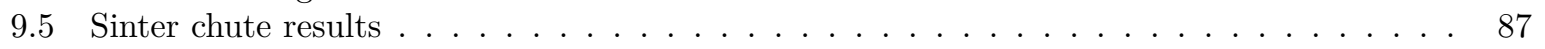

$\begin{array}{ll}10 \text { Blast Furnace } & 92\end{array}$

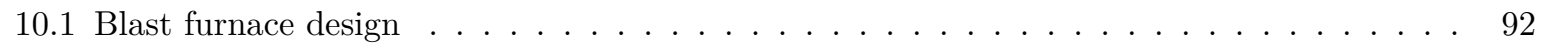

10.1 .1 Structure . . . . . . . . . . . . . . . . . . . . . . . . . . . 92

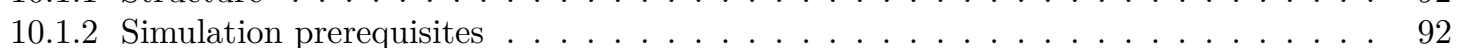

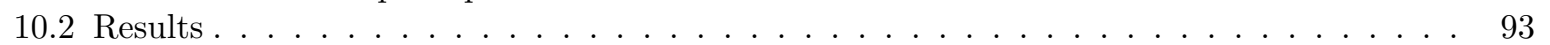

10.2 .1 Fluid velocity . . . . . . . . . . . . . . . . . . . . . . . . . 94

10.2 .2 Particle velocity $\ldots \ldots \ldots \ldots \ldots \ldots$

10.2 .3 Penetration depth $\ldots \ldots \ldots \ldots \ldots \ldots \ldots$

\begin{tabular}{lll}
\hline $\mathrm{V}$ Conclusion & 98 \\
\hline
\end{tabular}

\begin{tabular}{|lr}
\hline A Powder Technology journal paper & 100
\end{tabular} 
\begin{tabular}{ll}
\hline Bibliography & 111
\end{tabular}

\begin{tabular}{ll}
\hline About the author & 120
\end{tabular} 


\section{Abstract}

Numerous industries process particles. In this work, we focused on how to efficiently picture the behaviour of particles by means of numerical simulations, laboratory experiments, and Artificial Neural Networks (ANNs).

Particle-particle contact laws and particles size distributions determine the macroscopic results in Discrete Element Method (DEM) simulations. Commonly, contact laws depend on semi-empirical parameters which are difficult to obtain by direct microscopic measurements.

To clarify this aspect, we present the related elements of the DEM theory. The ANN theory is also introduced to demonstrate ANN effectiveness towards the solution of inverse problems with non linear regression.

Later, we describe the series of small scale DEM simulations with different sets of particle-based simulation parameters and particle distributions, which we performed. The macroscopic results of these simulations were used to train dedicated feed-forward ANNs by the backward propagation reinforcement algorithm. Concurrently, the bulk behaviours of raw particles were characterized by means of macroscopic laboratory experiments. These particles were those commonly used by metallurgical industries. At this point, the relationship between macroscopic results and microscopic DEM simulation parameters could be investigated.

We subsequently utilized this artificial neural network to predict the macroscopic ensemble behaviour in relation to additional sets of particle-based simulation parameters and particle distributions. By this method, a comprehensive database was established, relating particle-based simulation parameters to macroscopic ensemble output. If compared to an experiment of a specific granular material, this database identifies valid sets of DEM parameters which lead to the same macroscopic results as observed in the experiments. Finally, we applied the results of this method of DEM parameter identification to two industrial scale processes of steel production. 


\section{Zusammenfassung}

Viele Industriezweige verarbeiten granulare Medien. Diese Arbeit beschreibt wie das Verhalten von Partikeln über numerische Simulationen, experimentelle Laborversuche, und künstlichen neuronale Netze (KNN) abgebildet werden kann. Die makroskopischen Simulationsergebnisse der Diskreten Elemente Methode werden durch die gewählten Kontaktmodelle und die Größenverteilung der Partikel bestimmt. üblicherweise benötigen die verwendeten Modelle semi-empirischen Parameter, welche nur schwierig durch direkte mikroskopische Messungen ermittelt werden können.

Um diesen Aspekt zu verdeutlichen, werden die relevanten Bereiche der DEM Theorie erläutert. Die Theorie hinter KNN wird eingeführt um deren Effektivität zur Lösung von inversen Problemen mit nichtlinearer Regression zu demonstrieren. Anschließend werden kleinere DEM Simulationen beschrieben, welche mit unterschiedlichen Parametern und Größenverteilungen durchgeführt wurden.

Die makroskopischen Ergebnisse dieser Simulationen wurden dazu verwendet um feed-forward KNNs über Rückpropagierung zu trainieren.

Zeitgleich wurde das Verhalten von reinen Partikeln durch makroskopische Laborexperimente charakterisiert. Dafür wurden für die Metallindustrie übliche Partikel verwendet. Der Zusammenhang zwischen den makroskopischen Ergebnissen und mikroskopischen DEM Simulationen konnte darauf hin untersucht werden. Die künstlichen neuronalen Netze wurden anschließend verwendet um das makroskopische Verhalten.

Durch diese Methode wurde eine umfassend Datenbank erstellt, welche Parameter von Partikel Simulationen mit makroskopsichen Ausgaben verknüpft. Diese Datenbank kann nun für ein Experiment mit bestimmten granularen Material eine gültige Auswahl von DEM Parametern ermitteln werden. Die Methode wurde zum Abschluss dazu verwendet DEM parameter für zwei industrielle Prozesse bei der Stahlerzeugung zu identifizieren. 


\section{Acknowledgement}

This study was funded by the Christian Doppler Forschungsgesellschaft, Primetals Technologies, and Voestalpine Stahl. The author gratefully acknowledges their financial support.

Further, I would like to thank my family, Daniela, Fabrizio, and Nicolo', for the sentimental support over the last eight years of university.

A special thank to my supervisor Stefan Pirker, without whom this work would have not been possible. Many thanks also to Stefano Sandrone, who convinced me to start the $\mathrm{PhD}$ here in Linz. I would also like to thank Luca Flessati, who was struggling as me to obtain a $\mathrm{PhD}$, but he found the time to share some adventures with me.

Moreover, I feel grateful to my colleagues, especially Richard Berger, Bernhard König, Daniel Nasato, Daniel Queteschiner, Gerhard Holzinger, and Philip Hörmandinger, for their help during these years. I would also like to thank the employees of the Institut für Strömungslehre und Wärmeübertragung. Many thanks also for my first supervisor Christoph Kloss, who introduced me to the particles world.

I am also indebted to my friends Danilo Ansaloni and Fabio Molinari. Danilo not only walked me through the difficulties of advanced coding, but especially is a good friend and always believes in me. Fabio not only helped me founding a job after the $\mathrm{PhD}$, but he also remembered me that I should spend less time in office and more time meeting new people.

A last thank to everybody, sometimes including myself, who did not believe in me. My willingness to demonstrate I could do it nevertheless helped me to go through many difficult times. 


\section{Publications}

\section{Paper in SCI-Expanded-Zeitschrift}

Benvenuti L., Kloss C., Pirker S. (2016), Identification of DEM simulation parameters by Artificial Neural Networks and bulk experiments, Powder Technology.

\section{Paper in Tagungsband (referiert)}

Benvenuti L., Kloss C., Pirker S. (2015), Characterization of DEM particles by means of artificial neural networks and macroscopic experiments, Proceedings of the 16th International Conference on Engineering Applications of Neural Networks (EANN 2015), Rhodes, Greece.

Benvenuti L., Kloss C., Pirker S. (2015), Characterization of Particle Based Simulation Parameters by means of Artificial Neural Networks. Proceedings of Minisymposium Verfahrenstechnik, Wien, Austria.

Benvenuti L., Kloss C., Pirker S. (2015), DEM Parameter Identification by Means of Artificial Neural Network for Iron Ore Sintering, Proceedings of the 11th International Conference on CFD in the Oil \& Gas, Metallurgical and Process Industries (CFD2015), Melbourne, Australia.

Benvenuti L., Kloss C., Pirker S. (2015), DEM Particle Characterization by Artificial Neural Networks and Macroscopic Experiments, Proceedings of the 4th International Conference on Particles, Barcelona, Spain.

Benvenuti L., Aigner A., Queteschiner D., Pirker S., Kloss C. (2014), Establishing the predictive capabilities of DEM simulations: sliding and rolling friction coefficients of non-spherical particles, Proceedings of the 10th International Conference on CFD in the Oil \& Gas, Metallurgical and Process Industries (CFD2014), Trondheim, Norway.

Pirker S., Schiochet Nasato D., Benvenuti L., Kloss C., Schneiderbauer S. (2014), Simulation partikelbeladener Strömungen: Diskrete, kontinuierliche und hybride Modellierungsansätze, Proceedings of Danninger H., Kestler H., Kolaska H. (Eds.): Neue Horizonte in der Pulvermetallurgie - Werkzeuge, Produkte und Verfahren, Series Pulvermetallurgie in Wissenschaft und Praxis, Volume 30. 


\section{List of Figures}

1.1 Materials . . . . . . . . . . . . . . . . . . . . . . . . . . 3

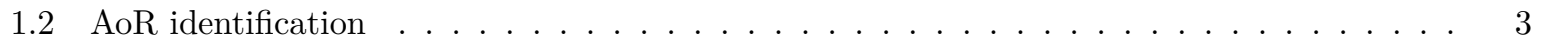

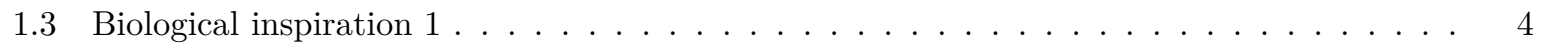

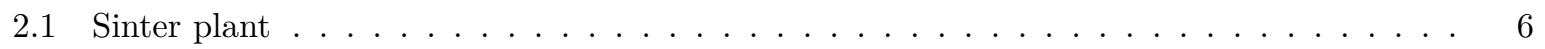

2.2 Blast furnace scheme $\ldots \ldots \ldots \ldots \ldots \ldots \ldots \ldots \ldots$

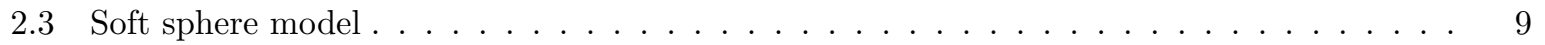

2.4 DEM simulation $\ldots \ldots \ldots \ldots \ldots$

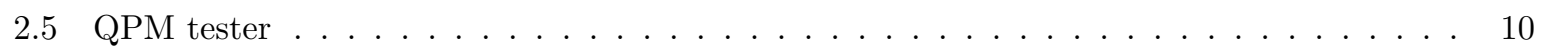

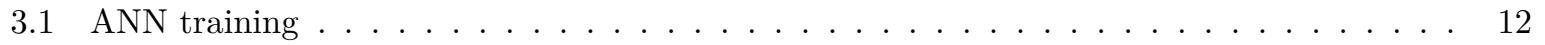

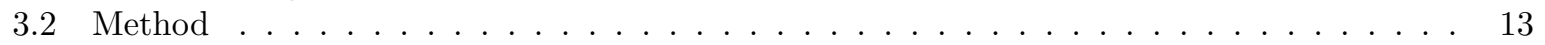

3.3 Materials investigated in this work. . . . . . . . . . . . . . . . . . . . 14

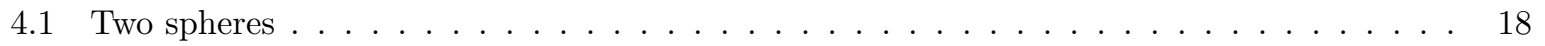

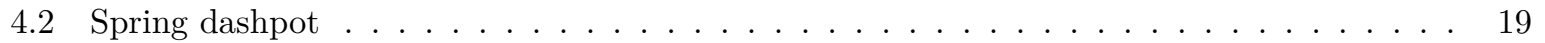

4.3 Unresolved CFD-DEM . . . . . . . . . . . . . . . . . . . . . . . . . . . . . . . . . . . . 21

4.4 Particle in a fluid . . . . . . . . . . . . . . . . . . . . . . . . . . . . . . . 22

4.5 Computational CFD-DEM steps $\ldots \ldots \ldots \ldots \ldots \ldots \ldots \ldots \ldots$

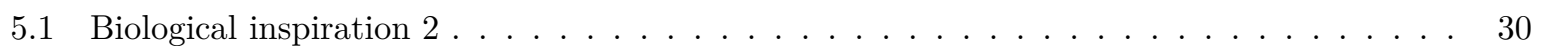

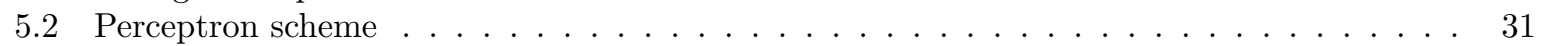

5.3 Double moon scheme $\ldots \ldots \ldots \ldots \ldots$. . . . . . . . . . . . . . . . . . . 31

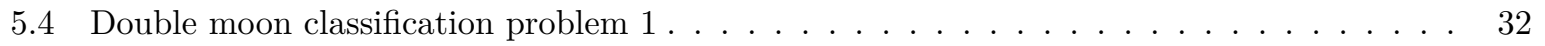

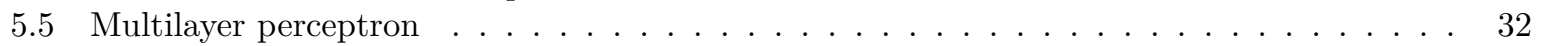

5.6 FF Backpropagation $\ldots \ldots \ldots \ldots \ldots \ldots \ldots \ldots \ldots \ldots \ldots$

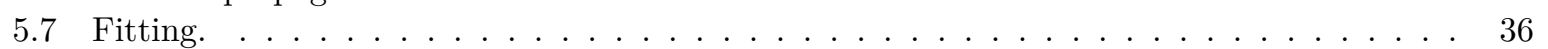

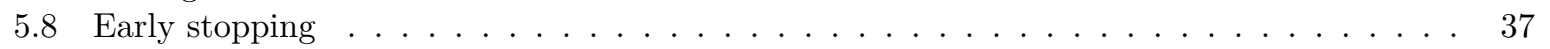

$6.1 \quad$ AoR experimental tester. . . . . . . . . . . . . . . . . . . . . . . . . 42

6.2 AoR large scale test $\ldots \ldots \ldots \ldots \ldots \ldots$. . . . . . . . . . . . . . . . . . . 43

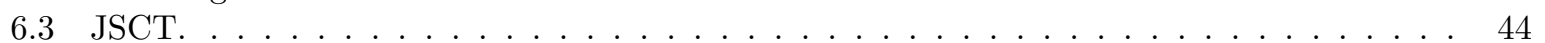

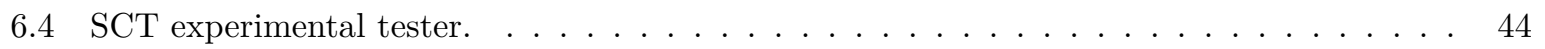

6.5 Experimental shear cell tester stress path $\ldots \ldots \ldots \ldots \ldots \ldots \ldots$

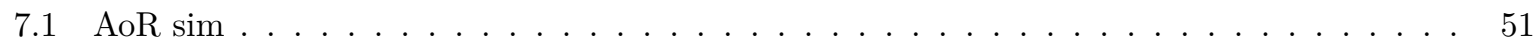

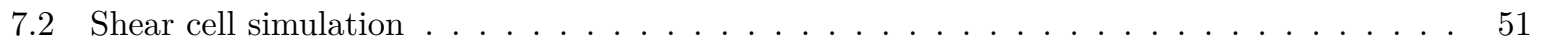

7.3 Numerical shear cell tester stress path $\ldots \ldots \ldots \ldots \ldots \ldots \ldots \ldots$. . . . . . . . . . . 53

7.4 Effect of the variation of the friction coefficients $\ldots \ldots \ldots \ldots \ldots \ldots$. . . . . . . . 54

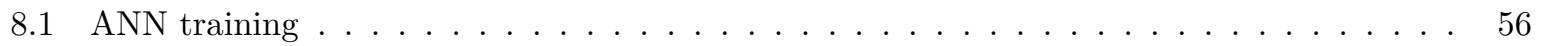


8.2 ANN Scheme . . . . . . . . . . . . . . . . . . . . . . . . . 57

8.3 Comparison between prediction of the trained ANN and full DEM simulation . . . . . . . 57

8.4 Regressions comparison for the $\mu_{p s h} \ldots \ldots \ldots \ldots \ldots \ldots$

8.5 Regressions comparison for the AoR . . . . . . . . . . . . . . . . . . . . . . . . 60

8.6 Method . . . . . . . . . . . . . . . . . . . . . . . . 62

8.7 SCT parameter space plots 2 . . . . . . . . . . . . . . . . . . . . . . . 64

8.8 SCT box plots for sinter fine . . . . . . . . . . . . . . . . . . . . . . . . 66

8.9 Density plot comparison of SCT results . . . . . . . . . . . . . . . . . . . . . 67

8.10 AoR valid values plots . . . . . . . . . . . . . . . . . . . . . . . 68

8.11 Merge parameter space plots of the sinter fine . . . . . . . . . . . . . . . . . . . . 70

8.12 Coke coarse . . . . . . . . . . . . . . . . . . . . . . . . . . 73

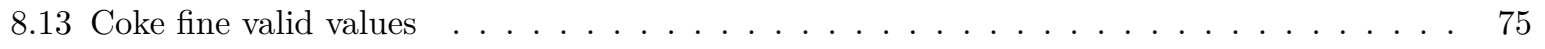

8.14 Iron ore coarse valid values . . . . . . . . . . . . . . . . . . . . . . . . . 77

8.15 Iron ore fine valid values . . . . . . . . . . . . . . . . . . . . . . . . . . . . . . . . . . . 78

8.16 Limestone coarse valid values . . . . . . . . . . . . . . . . . . . . . . . . . . . 80

8.17 Limestone fine valid values . . . . . . . . . . . . . . . . . . . . . . . . . . . . . . . . . . . 81

8.18 Sinter coarse valid values $\ldots \ldots \ldots \ldots$. . . . . . . . . . . . . . . . . 82

9.1 Sinter chute orto layout . . . . . . . . . . . . . . . . . . . . . . . . . 86

9.2 Sinter chute box $\ldots \ldots \ldots \ldots \ldots \ldots$

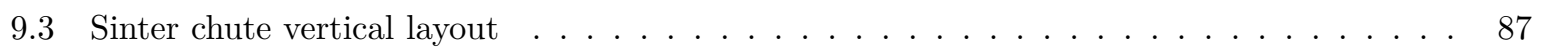

9.4 Sinter chute simulation layers . . . . . . . . . . . . . . . . . . . . . . . . . . 87

9.5 Sinter bar plot . . . . . . . . . . . . . . . . . . . . . . . . . . . . . . . . . . . . . . . 88

9.6 Simulation screenshots . . . . . . . . . . . . . . . . . . . . . . . . . . 89

9.7 Particle size distribution during the simulation 1 . . . . . . . . . . . . . . . . . . . . . . 90

9.8 Particle size distribution during the simulation 2 . . . . . . . . . . . . . . . . . . . . . . . 91

10.1 Schematics of the blast furnace investigation. . . . . . . . . . . . . . . . . . . . . . . 92

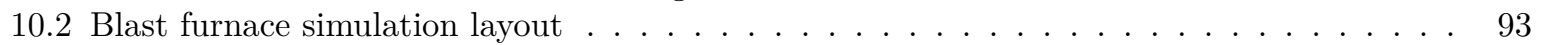

10.3 Vertical slice of fluid velocity . . . . . . . . . . . . . . . . . . . . . . . . . . . . . . . 9 94

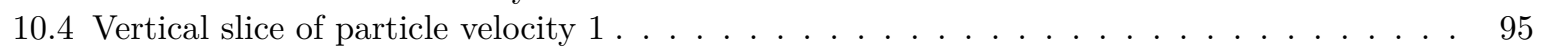

10.5 Vertical slice of particle velocity 2 . . . . . . . . . . . . . . . . . . . . . . . . . 96

10.6 Raceway penetration depth on the horizontal . . . . . . . . . . . . . . . . . . . . . . . . . 97 


\section{List of Tables}

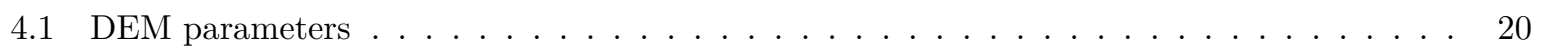

$5.1 \quad$ Regression methods comparison. $\ldots \ldots \ldots \ldots \ldots \ldots \ldots \ldots$

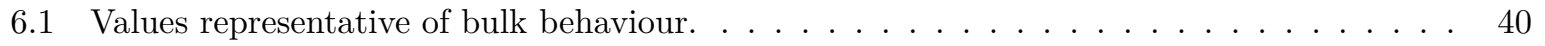

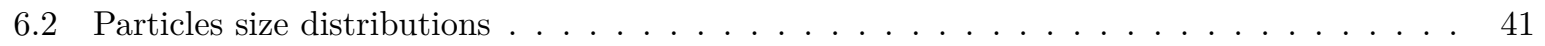

6.3 AOR experimental values. . . . . . . . . . . . . . . . . . . . . . . . . . . . 42

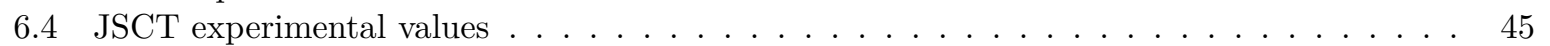

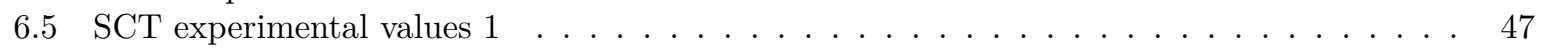

6.6 SCT experimental values $2 \ldots \ldots \ldots \ldots \ldots \ldots$

$6.7 \quad$ SCT experimental values $3 \ldots \ldots \ldots \ldots$

7.1 DEM fixed input values $\ldots \ldots \ldots \ldots \ldots \ldots \ldots \ldots \ldots \ldots$

7.2 DEM variable input values $\ldots \ldots \ldots \ldots \ldots \ldots \ldots \ldots \ldots \ldots$

8.1 Values of linear relationship between considered variables . . . . . . . . . . . . . 55

8.2 Weights and biases table for the coefficient of internal friction for sinter fine . . . . . . . 58

8.3 Weights and biases table for sinter fine AoR $\ldots \ldots \ldots \ldots \ldots \ldots \ldots$

8.4 Regression methods quantitative comparison. . . . . . . . . . . . . . . . . . . . . . 61

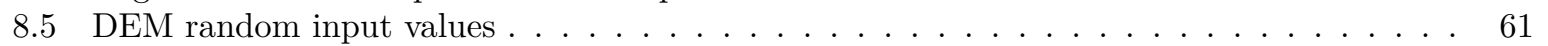

$8.6 \quad$ Valid DEM values for sinter fine $\ldots \ldots \ldots \ldots$

8.7 Weights and biases table for coefficient of internal friction . . . . . . . . . . . . . . 71

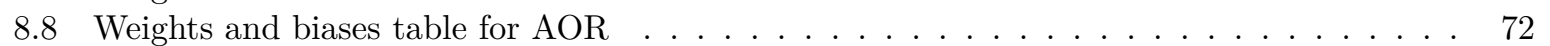

8.9 Valid DEM values for coke coarse $\ldots \ldots \ldots \ldots \ldots \ldots$. . . . . . . . . . . . . 72

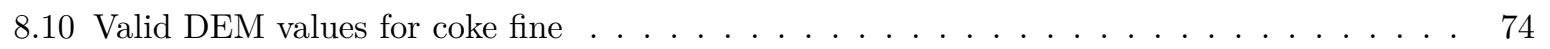

8.11 Valid DEM values for iron ore coarse $\ldots \ldots \ldots$. . . . . . . . . . . . . . 76

8.12 Valid DEM values for iron ore fine . . . . . . . . . . . . . . . . . . . . . 76

8.13 Valid DEM values for limestone coarse $\ldots \ldots \ldots$. . . . . . . . . . . . . . . . . . 79

8.14 Valid DEM values for limestone fine $\ldots \ldots \ldots$. . . . . . . . . . . . . . 79

8.15 Valid DEM values for sinter coarse $\ldots \ldots \ldots \ldots \ldots$. . . . . . . . . . . 83

9.1 Particles distribution at the inlet $\ldots \ldots \ldots \ldots \ldots \ldots \ldots \ldots$

10.1 DEM fixed input values for the blast furnace simulation. . . . . . . . . . . . . 93 


\section{Acronyms}

\begin{tabular}{|c|c|c|c|}
\hline ANN & artificial neural network & MLPNN & $\begin{array}{l}\text { FF Multilayer Perceptron Neural } \\
\text { Networks }\end{array}$ \\
\hline ANNs & artificial neural networks & & \\
\hline AoR & static angle of repose (-) & $\mu_{i e}$ & coefficient of internal friction (-) \\
\hline CFD & computational fluid dynamics & $\mu_{s}$ & coefficient of sliding friction (-) \\
\hline CoR & coefficient of restitution (-) & $\mu_{p s h}$ & $\begin{array}{l}\text { steady-state flow/pre-shear coefficient } \\
\text { of internal friction (-) }\end{array}$ \\
\hline $\begin{array}{l}\mathscr{E}(\mathrm{w}) \\
\text { dCylDp }\end{array}$ & $\begin{array}{l}\text { Cost function } \\
\text { geometry factor (-) }\end{array}$ & $\mu_{s h}$ & $\begin{array}{l}\text { incipient flow/shear coefficient of } \\
\text { internal friction (-) }\end{array}$ \\
\hline$\Delta t$ & time step $(\mathrm{s})$ & $\mu_{r}$ & coefficient of rolling friction (-) \\
\hline DEM & discrete element method & $\eta$ & learning-rate parameter \\
\hline DEMs & discrete element methodS & $n$ & normal \\
\hline DoE & Design of Experiments & $\nu$ & Poisson's ratio (-) \\
\hline $\mathbf{E}$ & Young's modulus $(\mathrm{Pa})$ & $\begin{array}{l}p-p \\
R^{2}\end{array}$ & $\begin{array}{l}\text { particle-particle } \\
\text { coefficient of determination }(-)\end{array}$ \\
\hline FF & Feedforward & $\mathbf{R B F}$ & Radial gaussian basis function \\
\hline$k$ & coefficient of elasticity (-) & $\rho_{b}$ & bulk density $\left(\mathrm{kg} / \mathrm{m}^{3}\right)$ \\
\hline$k_{n}$ & normal coefficient of elasticity (-) & $\rho_{p}$ & particle density $\left(\mathrm{kg} / \mathrm{m}^{3}\right)$ \\
\hline$\gamma$ & coefficient of viscosity (-) & SCT & Schulze shear cell tester \\
\hline$\gamma_{n}$ & normal coefficient of viscosity (-) & $\sigma_{n}$ & normal stress $(P a)$ \\
\hline$k_{t}$ & tangential coefficient of elasticity (-) & $t$ & tangential \\
\hline$\gamma_{t}$ & tangential coefficient of viscosity (-) & $\tau_{\%}$ & shear stress percentage $(\%)$ \\
\hline JSCT & Jenike shear cell tester & $R_{i}$ & radius $(\mathrm{m})$ \\
\hline \multicolumn{2}{|c|}{$\begin{array}{l}\text { LIGGGHTS LAMMPS improved for general } \\
\text { granular and granular heat transfer } \\
\text { simulations }\end{array}$} & $\begin{array}{l}\xi_{n} \\
\xi_{t}\end{array}$ & $\begin{array}{l}\text { normal overlap } \\
\text { tangential overlap }\end{array}$ \\
\hline
\end{tabular}





\section{Part I}

\section{Introduction}




\section{Chapter 1}

\section{Prologue}

Particles in various forms - ranging from raw materials to food grains and pharmaceutical powders - play a major role in a variety of industries, including process industry and metallurgy. In his book, Holdich [71] stated that "between 1 and $10 \%$ of all the energy is used in comminution, i.e. the processes of crushing, grinding, milling, micronising". Many methods have been developed to study particles. For instance, Discrete Element Methods (DEMs), "a special class of numerical schemes for simulating the behavior of discrete, interacting bodies", are widely used to simulate particle behaviour in these granular processes (Cleary and Sawley [37]).

\section{$1.1 \quad$ Metallurgical industry}

In this thesis, we focused on the granular materials the metallurgical industry handles, especially, in two processes:

- sinter cooling,

- pig ore smelting through a blast furnace.

In the first process we were interested about a sinter chute segregator, or more specifically, about the size distribution of the particles at its exit.

In the second process, we wanted to investigate the raceway zone of the blast furnace, both its formation and its evolution.

\subsection{Challenges}

In fact, gravels, Fig. 1.1a, or granular particles in general, are far from being a well-defined and easy to characterize material, like for instance a steel beam, Fig. 1.1b. For continuous materials simulation parameters are readily available. In case of a pile of particles the sum of discrete particle properties determines the pile's macroscopic behavior (e.g., angle of repose).

In discrete particle simulations particle based parameters (e.g., contact parameters) determine the macroscopic behavior of the ensemble. Unfortunately, particles are not uniform and particle based simulation parameters are difficult to obtain, and also depends on the numerical shape (polyhedral, multi-spheres, and simple spheres). 


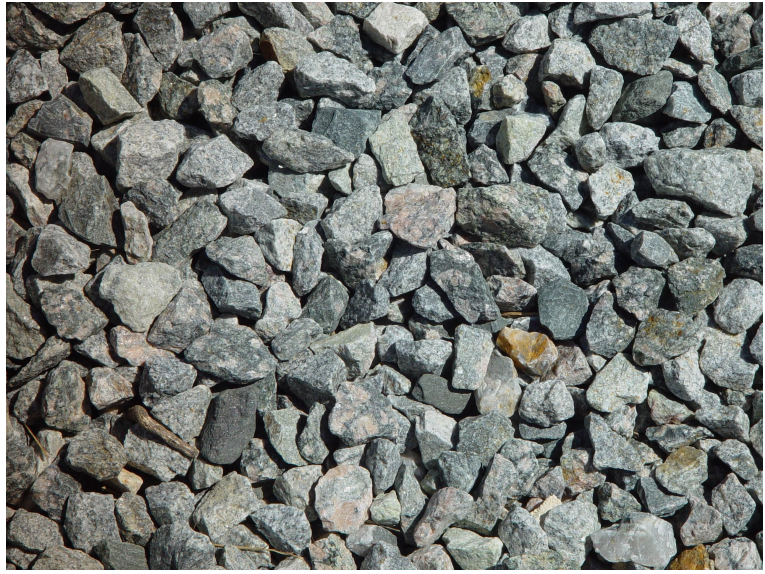

(a) Gravel, granular material.

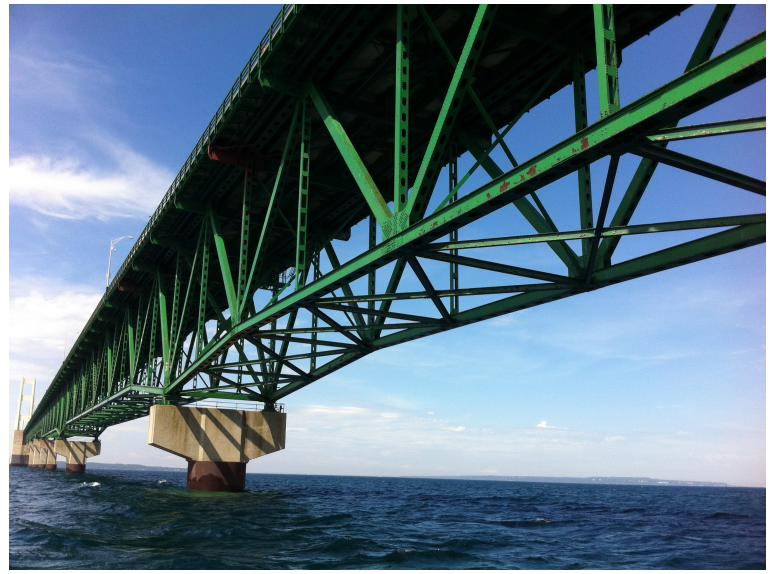

(b) Steel, continuous material.

Figure 1.1: Visual comparison between granular and continuos materials [140].

\subsection{Calibration}

A set of experimental and numerical solutions, together with artificial neural networks to regress the inverse problem, could improve the accuracy and the range of applicability of the characterization of particles properties, and reduce the computational costs. The Discrete Element Method requires parameters for the individual contact, but to characterize every particle is prohibitive.

We needed to find average contact parameters that could lead to the expected bulk effect. We could start with an example of piled particles, more specifically called the drained angle of repose. This angle of repose, see Fig. 1.2, characteristic of the bulk macroscopic behaviour of the ensemble, is originated from the microscopic characteristics of each particle. Once measured the bulk parameter value, through

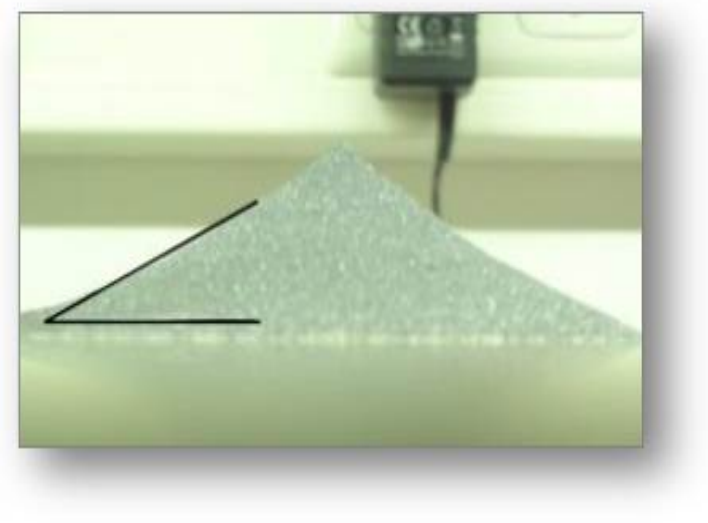

(a) Silibeads angle of repose.

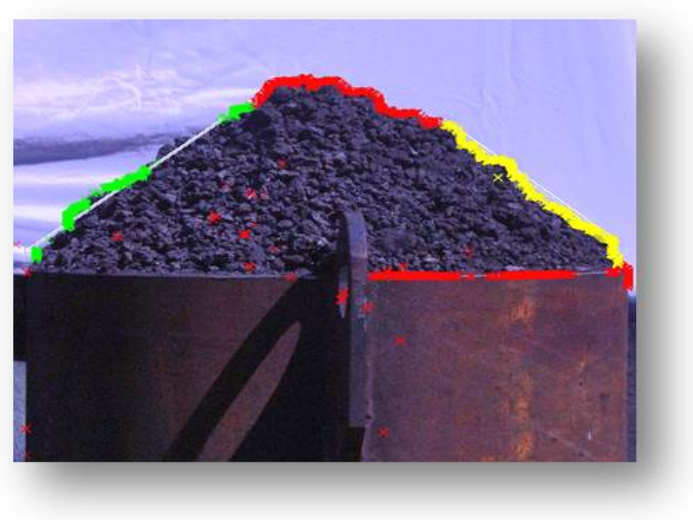

(b) Sinter pellets angle of repose.

Figure 1.2: Example of bulk solids material characterization: angle of repose identification.

calibration we could obtain the individual contact parameters:

1. we chose initial set of parameters;

2. we performed a DEM simulation; 
3. we compared macroscopic DEM simulation results with experiments;

4. if they matched we ended the loop, otherwise we started again from 1 with new parameters.

By our calibration procedure we could obtain valid sets of particle based simulation parameters in an extremely time consuming way, because in each control loop we would have to perform a complete DEM simulation. In fact, a feasibility study from Benvenuti et al. [18] required a total of 9.900 days on a 32 core machine to reliably complete a calibration procedure. However, the evaluation of a large number of parameter sets can not be considered essential, nor necessary. Our aim was rather to determine the sensitivity of the macroscopic bulk behavior with respect to individual particle based parameters. This could be realized efficiently by artificial neural networks, see Fig. 1.3

\subsection{Neural Network Calibration}

After the original work of y Cajal [143] we know that the human brain is composed of neurons and their connections. They receive inputs from receptive nerves and together elaborate an output response (e.g., to remove the hand from a hot surface). Similarly, in the artificial neural network we trained a

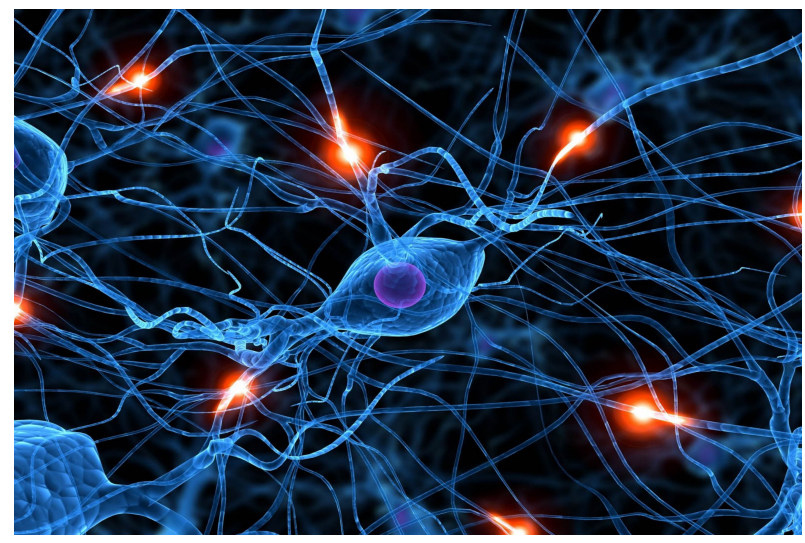

Figure 1.3: Biological inspiration 1 for the Artificial Neural Networks: a human neuron with the incoming electric signals [67].

series of neurons to solve a regression problem. The neurons had as inputs particle based parameters and as output the corresponding DEM simulation results. With both data the network was trained (i.e. individual neurons are weighted). Later, the trained neural network could be used to predict additional valid sets of particle based simulation parameters.

1. We trained a neural network by approximately 500 dedicated DEM simulations (time consuming);

2. we predicted another 6250000 combinations by the neural network (fast);

3. we checked the corrrectness of the neural network predictions (by comparing with experimental values).

\subsection{Parameters Identification}

In our experience, less than $1 \%$ of the predicted parameter sets lead to correct macroscopic results (i.e. 6.000 to 60.000 valid parameter sets). By this assessment of particle based simulation parameters we obtained valuable information about the dependence of bulk solid behavior on individual particle properties. First we could determine the validity range, mean and variance band of each input parameter. 
Next we could determine a probability density function for each input parameter. Then, we could investigate mutual dependencies. This calibration procedure was universal in a sense that the same artificial neural network could be harnessed for different macroscopic bulk behaviors. This effort was really necessary because the predictive capability of any DEM simulation strongly depends on the validity of the particle based simulation parameters.

Once we had reliable DEM parameters, we could use them for large scale DEM simulations. 


\section{Chapter 2}

\section{Motivation and Insufficiency of the State of the Art}

Biswas [24] states that the iron metal can be obtained by reduction of iron oxides, of which hematite and magnetite are the most common types. The most used apparatus to obtain pig iron is the blast furnace. Iron oxides are mined from earth crust. However, a blast furnace needs particles ranged between 7 and 25 millimiters [24], to grant a regular descent of the particle into its hearth. To reach the required specification, the extracted minerals are crushed and then sieved. Sieving divides the particles in two categories:

- lump ore, between 7 and 25 millimiters,

- fines, less than 7 millimiters.

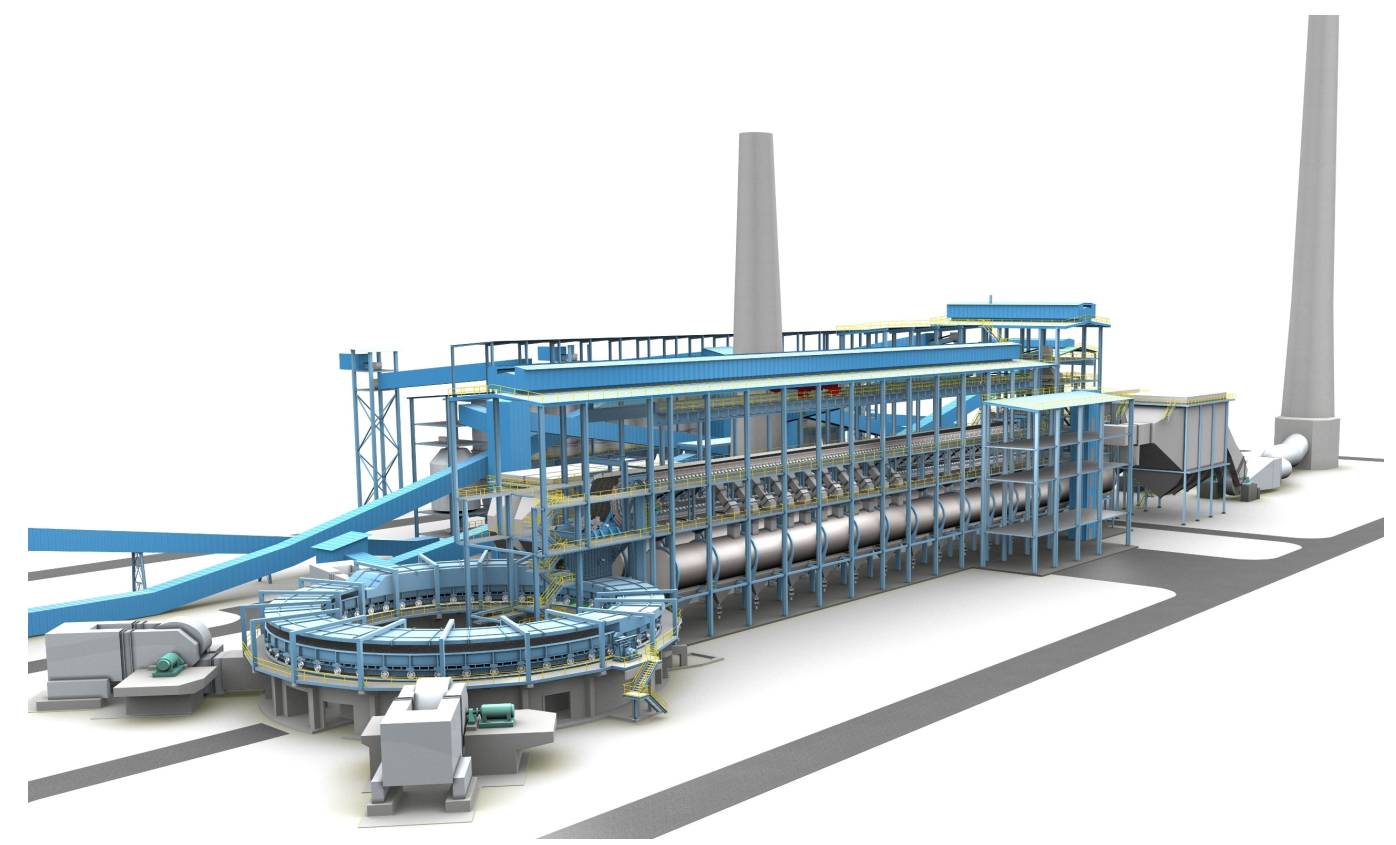

Figure 2.1: Sinter plant (Primetals GmbH). Fines are transformed into aggloramerates, ready for smelting.

Lump ore can be used directly for blast furnace smelting. Instead, fines need to be sintered (Fig. 2.1. 


\section{$2.1 \quad$ Sintering}

Sintering consists in partially melting the fines with limestone and coke.

The resulting granular material needs nonetheless to be cooled for transportation and storage. The metallurgical industry uses a sinter chute cooling system for this purpose, see Fig. 2.1, with many phases:

1. Hot particles are fed into a chute, which deposits the particles on a rotating system and segregates them, i.e. lays the large particles on the bottom and the small ones on top.

2. Air is injected from the bottom of the rotating system, cooling the particles.

3. The resulting particles larger than 25 millimiters are crushed, and reinserted in the flow.

4. The particles smaller than 7 millimiters are melted once more.

5. The particles between 7 and 25 millimiters are ready for blast furnace smelting.

In our thesis, we focused on (1), and the particles involved in this process.

\subsection{Blast furnace}

Well explained in the vast literature $([26])$, iron making consists in separating the iron from its chemical combination with oxygen. As of now, the blast furnace (Fig. 10.1a) is considered the most efficient process. It can be described as a countercurrent heat and oxygen exchanger. High temperature air, eventually in combination with pulverized coal, is inserted into the inferior section of the apparatus.

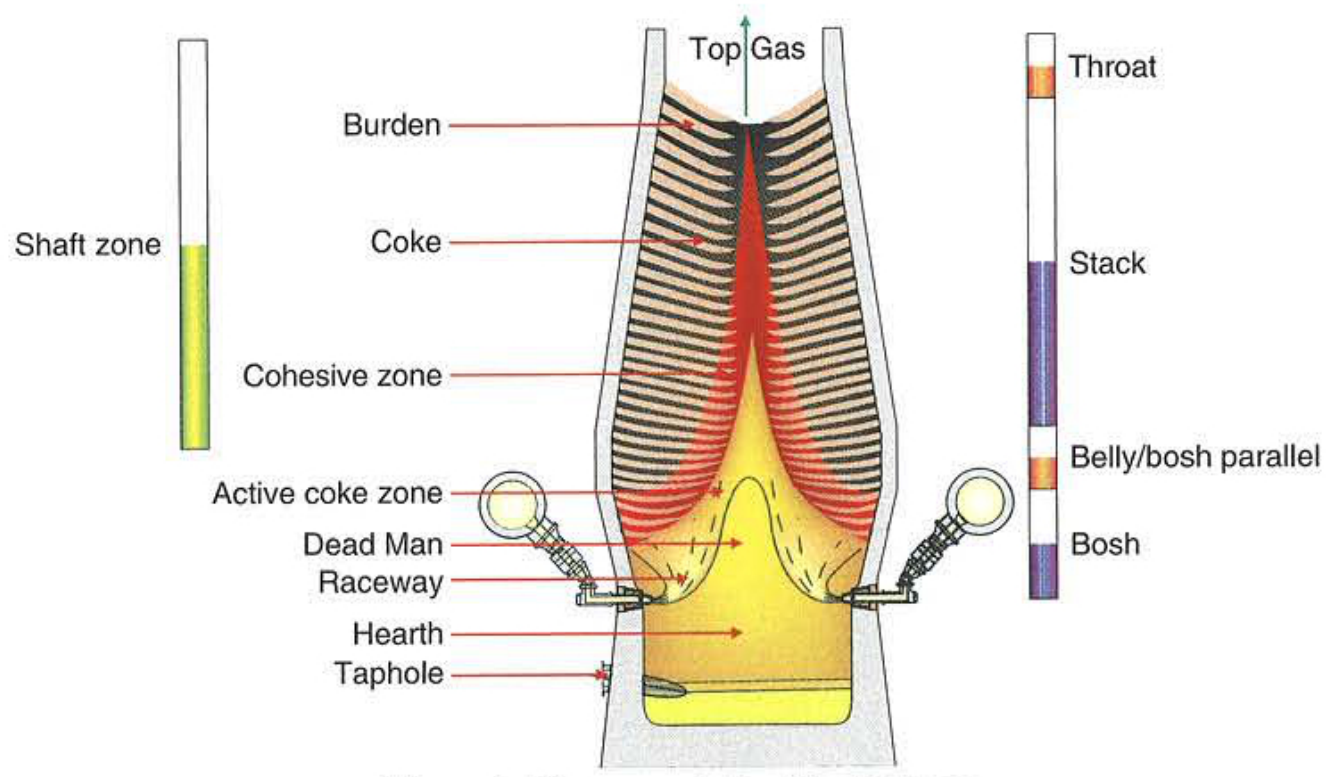

Figure 2.2: Blast furnace scheme [4]. Materials is inserted from the top and extracted at regular intervals from the taphole.

While rising, the gases provide the surronding particles with heat, and exits out the superior section of the furnace. Symmetrically, iron oxydes descend from the superior section and it is converted to metallic 
iron, which is collected into the inferior section.

\subsubsection{Blast furnace structure}

The blast furnace is a massive vertical apparatus, built with consistent amounts of refractory materials and a robust steel shell.

We can divide it in five sections from bottom to top:

1. hearth, where metal and slags are collected and tapped at regular intervals;

2. bosh, into which a special type of pipes, called tuyeres, insert the air at a temperature between $900^{\circ}$ and $1350^{\circ}$;

3. bosh parallel;

4. stack;

5. throat, where the solid charge of ore and coke is provided, and partially exhaust combustion gases exit;

The areas in front of each tuyere, which can be seen in Fig. 10.1a is called raceway and is the most active. We dedicated chapter 10 to the investigation of its behaviour.

\subsubsection{Blast furnace chemistry}

The most relevant chemical reactions inside the furnace are presented in Eq. 2.1

$$
\begin{aligned}
2 \mathrm{C}+\mathrm{O}_{2} & \rightarrow 2 \mathrm{CO}+\text { heat }, \\
\mathrm{Fe}_{2} \mathrm{O}_{3}+3 \mathrm{CO} & \rightarrow 2 \mathrm{Fe}+3 \mathrm{CO}_{2} .
\end{aligned}
$$

However, not only the combustion of coke inside the charge provides the heat necessary for iron melting. The air provided by the tuyeres is pre-heated through a combination of recycled exit combustion gases and methane.

\subsection{Materials characterization}

A univocal method to characterize the mentioned particles has so far not been established. From the experimental point of view, the main issues are the difficult setups and the general reliability and reproducibility of the tests. From the numerical point of view, no general procedure is available, and the existence of a mathematically unique solution describing macro/micro particle contact has yet to be proved.

Moreover, in a recent study, Krantz et al. [86 implied "that the dynamic properties of a powder cannot be applied to universally predict the static properties of a powder, and, likewise, the static properties cannot be used to predict dynamic properties".

\subsection{DEM Simulations}

Many authors usedDEM the discrete element method used in this work, to understand sintering processes [94, 97] and smelting [100, 105].

In their original formulation of DEM Cundall and Strack [42] allowed two particles to slightly overlap upon contact, see Fig. 2.3, and consequently they proposed repulsive forces in relation to this overlap distance. The force that particle $i$ exerts on particle $j$ is defined as: 


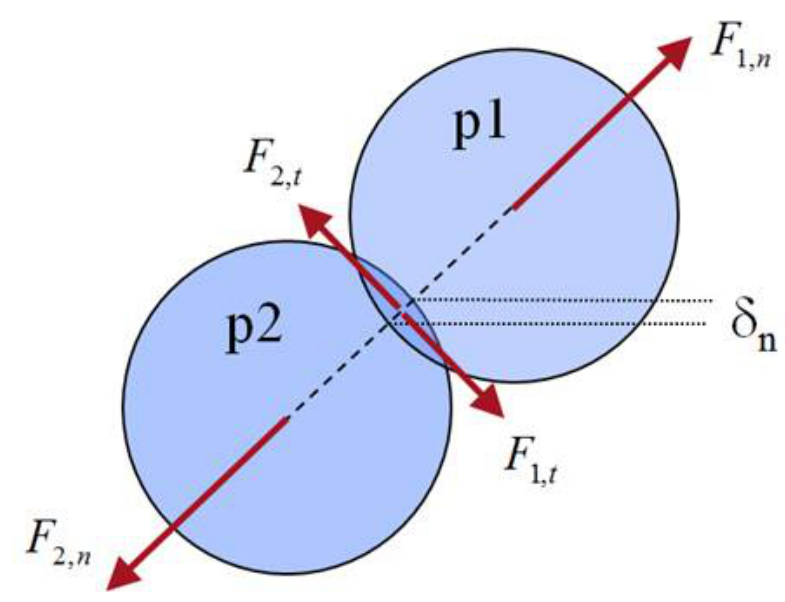

Figure 2.3: Soft sphere model schematics, as described by Holdich [71].

$$
m \ddot{x}_{i j}+c \dot{x}_{i j}+k x_{i j}=F_{i} .
$$

Their fundamental modelling concept of DEM has since been widely accepted in the literature and their soft sphere contact law has been developed further by numerous researchers (Renzo and Maio [116], VuQuoc and Zhang 138). With increasing computational resources, DEM simulations have become very popular giving rise to the development of commercial (e.g., $P F C 3 D$, used by Wensrich and Katterfeld [139]) and open-source softwares (e.g., LIGGGHTS Aigner et al. [3, Goniva et al. [51, Kloss and Goniva [82, Kloss et al. [83]). Soft-sphere DEM simulations of thousands of particles have been proven to faithfully model particle bulk behaviour, as in Fig. 2.4 (Hohner et al. [70]).

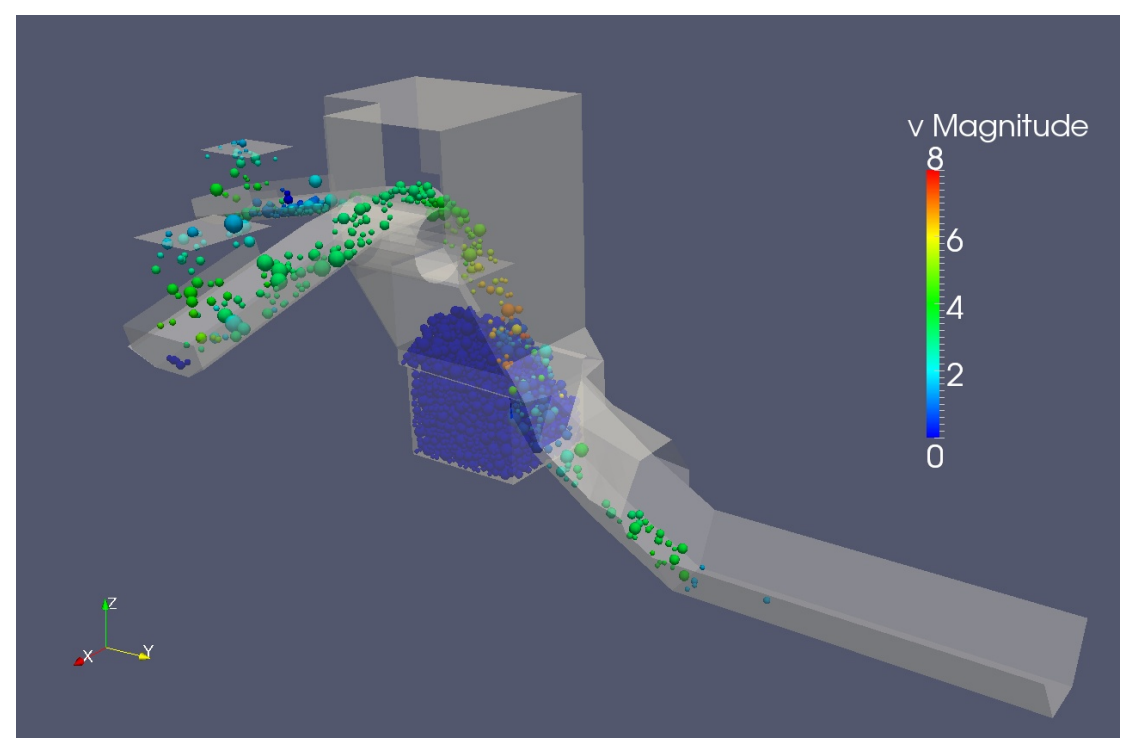

Figure 2.4: Example of a DEM simulation (www.cfdem.com). 


\subsection{Parameters}

In these macroscopic DEM simulations, the contact law kernel between a pair of particles determines the global bulk behaviour of the granular material (Ai et al. [1]). As a consequence, defining a correct contact law is of crucial importance for the predictive capability of DEM simulations. Since DEM contact laws are based on a set of semi-empirical parameters, correct contact law parameters must be defined for a given granular material or DEM simulations will fail (Combarros et al. [40]).

Identifying DEM contact law parameters is not a trivial task. Due to the huge number of particles in a granular material, it may be impractical to identify valid parameter sets by performing bilateral particle collision experiments. Furthermore, some contact law parameters such as the coefficient of rolling friction are purely empirical and cannot be determined by direct particle-to-particle measurements (Wensrich and Katterfeld [139]). Therefore, DEM contact law parameters (Table 4.1) are commonly determined by comparing the macroscopic outcome of large-scale DEM simulations with bulk experiments (Alenzi et al. [4). If DEM]simulation results disagree with bulk measurements, the set of contact law parameters must be adjusted until reasonable agreement is achieved, as explained in Section 1.5 .

However, this purely forward methodology of parameter identification is limited by the multi-dimensionality of the parameter space and the associated computational costs of the required DEM test simulations. Moreover, one parameter set which is valid for one bulk behaviour (e.g., angle of repose) might fail for another (e.g., shear tester).

\subsubsection{Direct Determination}

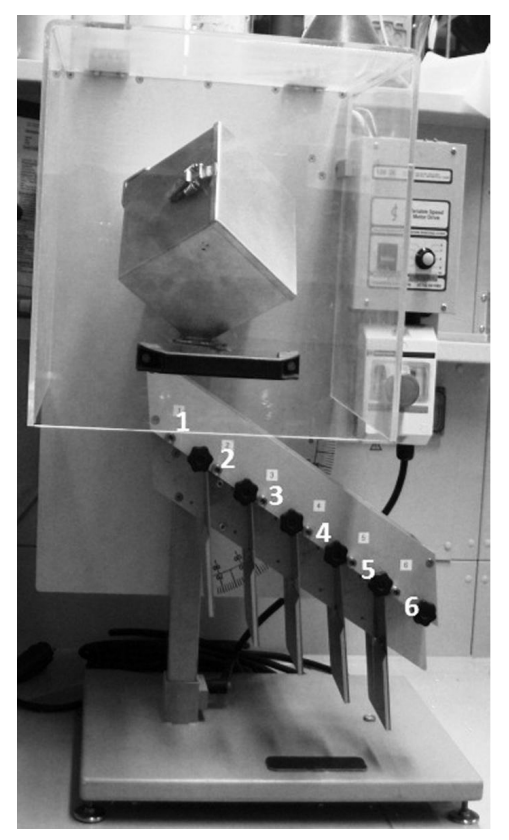

Figure 2.5: Quality in particulate manufacturing (QPM) segregation tester 40.

There are yet ways to determine contact parameters directly by measuring material properties or by performing particle based experiments, see e.g. Combarros et al. [40, Lommen et al. [92, Paulick et al. [107], as in Fig. 2.5

However, these methodologies are laborious, since they have to be performed for every new granular material prior to a DEM simulations. Especially for the already cited rolling friction parameter, it is arduous to link the rolling friction parameter to the non-sphericity of the particle. In literature (e.g., 
Combarros et al. [40]) is usually desumed from the angle of repose (AoR). However, in section 8.1 will be shown how this parameter accounts only for a portion of the final value.

Clearly, there is a need for an efficient method for identifying DEM contact law parameters, given a specific particle behaviour. 


\section{Chapter 3}

\section{Aim of this Thesis}

In our study, we harnessed Artificial Neural Networks ANNS in order to reduce the number of DEM test simulations required to characterize bulk materials for large scale simulations.

As the following chapters will show, we divided this task in two sections:

1. identification of DEM parameters, in part III.

2. applications, in part IV

\subsection{Aim for Neural Network}

ANNS have proven to be a versatile tool in analysing complex, non-linear systems of multi-dimensional input streams (Vaferi et al. [135], Witten et al. [141] and Haykin [67]). In our case, we fed an ANN with DEM contact law parameters as input and compared the output with the bulk behaviour predicted by a corresponding DEM simulation. The difference between ANN prediction and DEM prediction was used to train our specific ANN with a backward-propagation algorithm, comprising a limited number of DEM test simulations.

This ANN based regression was compared with two standardized regression methods, namely the

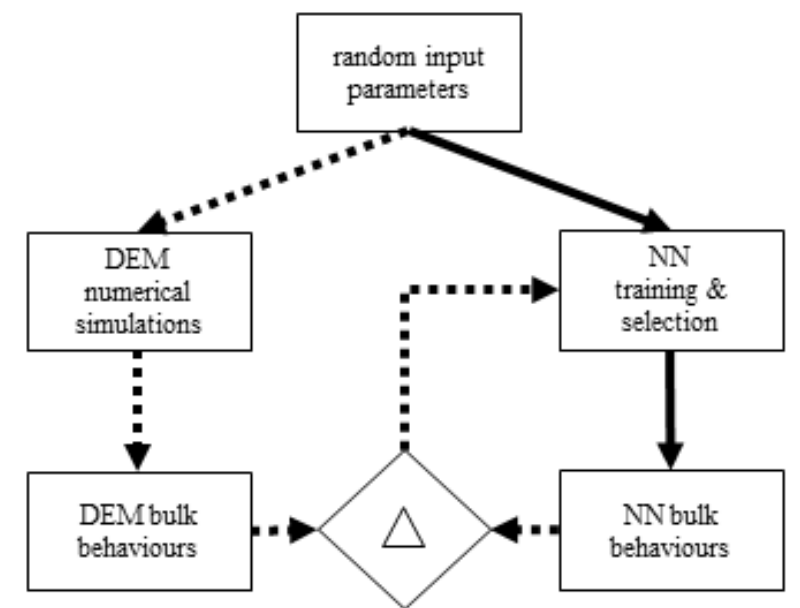

Figure 3.1: ANN training. Numerical simulations inputs and outputs are used to train the networks.

Bayesian linear and the Gaussian non linear. By comparing the fitting errors or each method, we were able to demonstrate that the ANN was the most effective. After the training phase, the ANN could then 
be used as a stand-alone prediction tool for the bulk behaviour of a granular material in relation to DEM contact law parameters, see Fig. 8.1.

In this study, we applied this parameter identification method to two different granular bulk behaviours, namely the angle of repose (AoR test and the Schulze shear cell (SCT) test. In both cases, we first trained a specific ANN using a number of DEM test simulations before we identified valid sets of DEM contact law parameters by comparing the stand-alone ANN predictions with corresponding bulk experiments. For both cases we obtained valid sets of contact law parameters, which we then compared to formulate a reliable contact law for a given granular material. We further showed that the same ANN could be used to characterize different granular materials, which have the same particle behaviour and could be modelled with the same contact law.

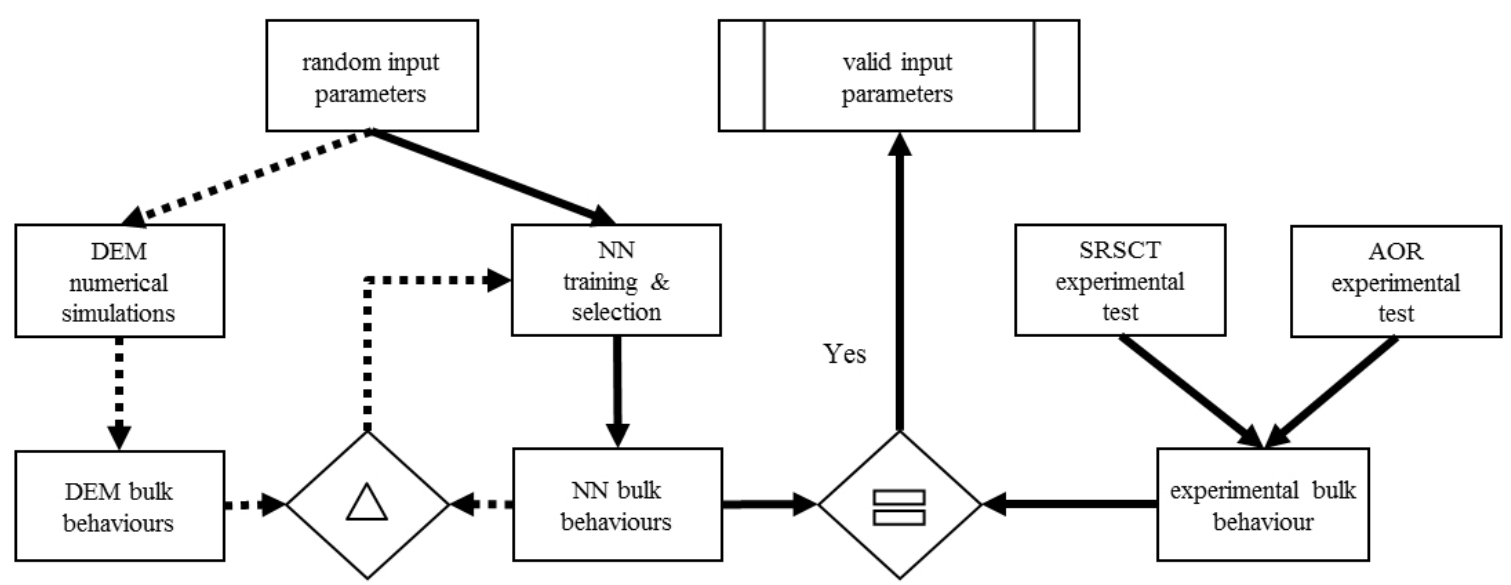

Figure 3.2: Method. In the training phase (dashed lines) $D E M$ simulations are performed with random initial input parameters. The behaviours obtained are used to train the Artificial Neural Networks $(A N N s)$ in a loop that continues until the difference between the outputs of each $A N N$ and its simulations is below the limit $(\Delta)$. In the parameters identification phase (solid lines) we identify valid input parameters by comparing $(=) A N N s$ and experimental behaviours.

\subsubsection{Applicability and scope}

The materials used for this work were coke, iron ore, limestone, and sinter fine, see Fig. 3.3. Their particles are cohesionless, see Chapter 6. Their amounts of open and closed pores are not negligible (see Yang [144), and thus the use of an Archimedean procedure to determine the correct particle density by direct measurement was not applicable. Possibly, a tomography for each particle would lead to the particle density (e.g., Gibson et al. [50]), but this analysis was infeasible: the necessary time for the the amount of material analysed was unreasonable. Further, a three-dimensional scan of each particle would have surely physically identified our particles, and made us able to confront them with other studies. The high shape dispersity made also this analysis impossible. We followed a different procedure, see Chapter 8 .

To avoid the macroscopic plastic deformations analysed by Harthong et al. 65. we checked for each simulation for its total duration that the packing density was lower than a defined limit. This threshold was given by the packing density of the close random packing with the same size distribution, as suggested and with the software provided by Baranau, Tallarek et al. [9, 10]. The experimental characterization was performed at environmental temperature and thus temperature was not considered in the numerical simulations. Chemical reactions and aging were also not part of the scope of this work. Further, due to the limited number of experiments realized, a restricted number of flow regimes were tested, and only one size distribution was considered. The authors are confident that these two limitations have 


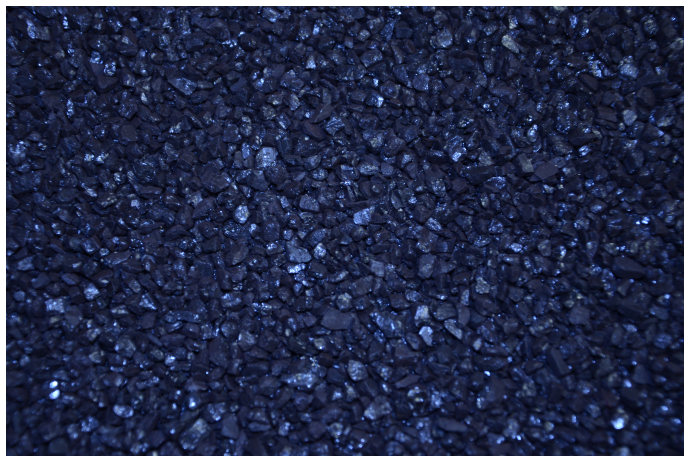

(a) Coke coarse.

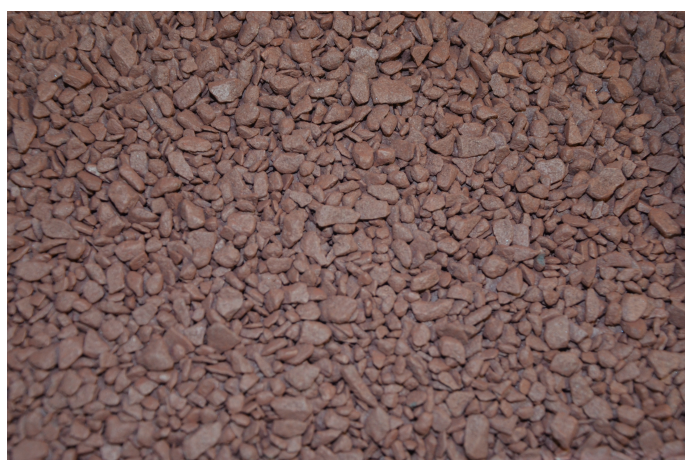

(c) Iron ore coarse.

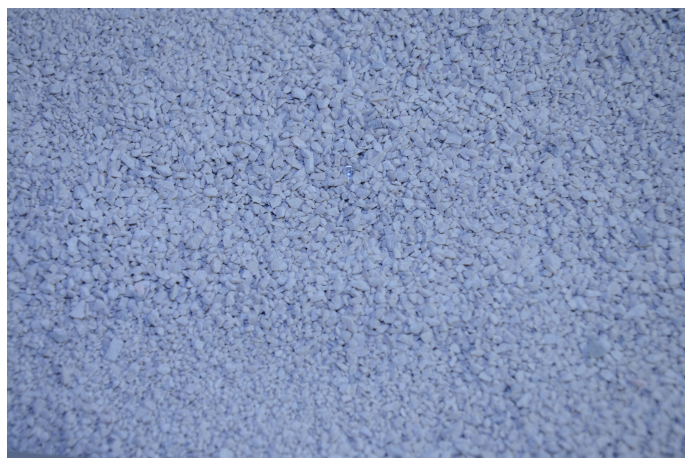

(e) Limestone coarse.

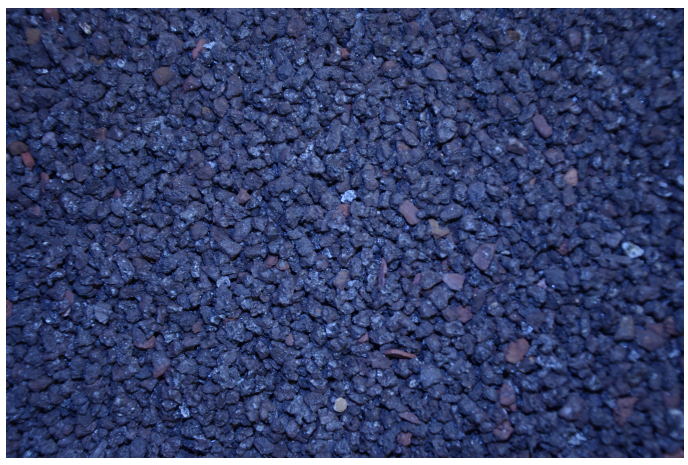

(g) Sinter coarse.

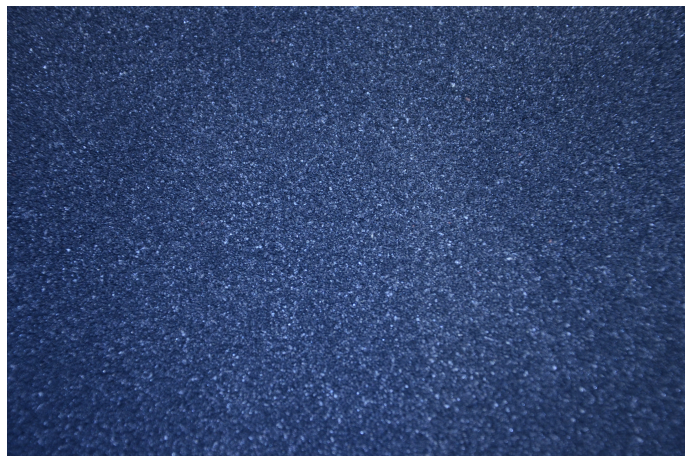

(b) Coke fine.

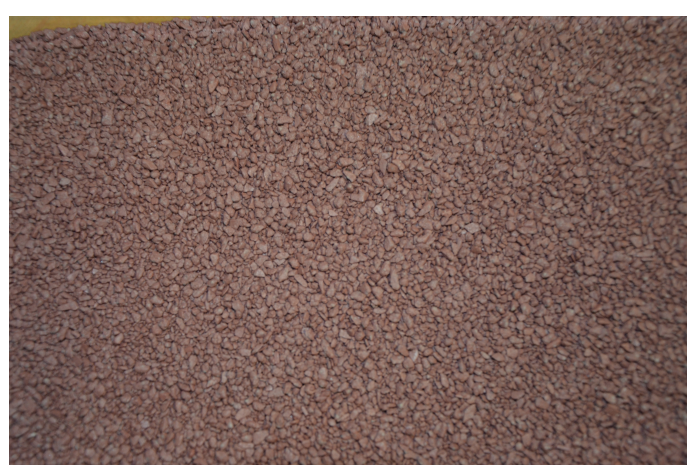

(d) Iron ore fine.

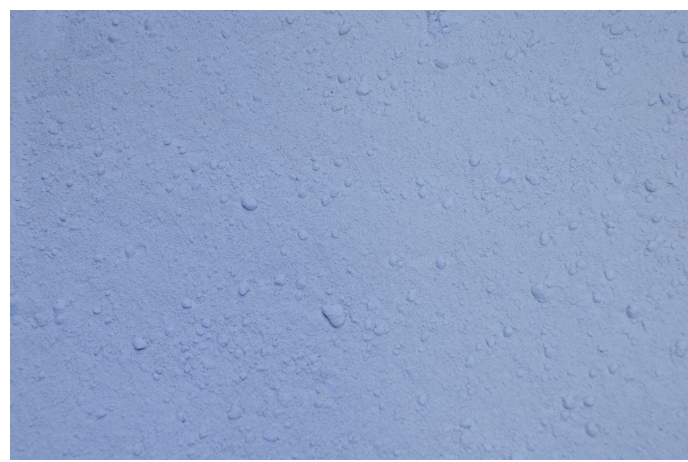

(f) Limestone fine.

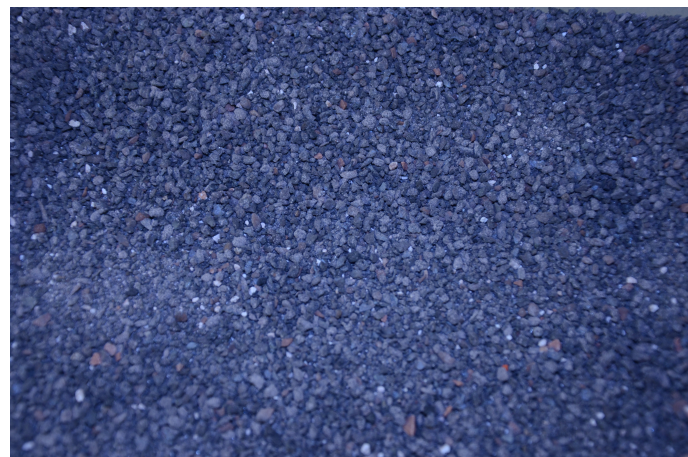

(h) Sinter fine.

Figure 3.3: Materials investigated in this work. 
not compromised the validity of the method. Rather, further investigations on these two aspects could again demonstrate its effectiveness. Notably, we chose an elastic model to picture the particle behaviour. If we try to identify parameters for an elastic contact model in a system of non-elastic particles, this $A N N$ approach will fail. So choosing appropriate contact models is an essential pre-requisite for contact parameter identification by means of $A N N$. Moreover, $A N N s$ can give indications about the correctness of the chosen model, see Chapter 8 


\section{Part II}

\section{Theory}




\section{Chapter 4}

\section{Discrete Element Method}

The discrete element method (DEM) is derived from Molecular Dynamics [112, 113].

Being a Lagrangian method, for each particle $i$ inside the domain, a DEM code follows the trajectory and calculates the force that particle $i$ exerts on particle $j$. The main forces involved are: gravity, contact forces due to collisions, and further interactions such as electrostatic, Van der Waals, cohesive forces and fluid-solid interactions in multiphase flows.

Two approaches can be implemented for this method:

- hard spheres,

- soft spheres.

The hard-sphere approach is well-suited for binary collisions, easy to calculate, but cannot predict multiparticle interactions. It is mainly used for dilute flows (e.g., Chialvo et al. [35], Feng and Musong [45], Kim and Lee [81, Sun and Sundaresan [129]), rather then in the dense flows we investigated.

For the simulations performed in a coupled particle-fluid environment, see Chapter 10, we used an unresolved CFD DEM approach, see 4.3

\subsection{Soft sphere approach}

The soft sphere approach considers the deformation of particles when they collide with each other. From the vector in Fig. 4.1 we can define after time $t$ the normal and tangential overlaps $\xi_{n}$ and $\xi_{t}$

$$
\begin{gathered}
\xi_{n}=R_{i}+R_{j}-\mathbf{n} \cdot\left(\mathbf{r}_{j}-\mathbf{r}_{i}\right), \\
\xi_{t}=\int_{0}^{t}\left|\mathbf{v}_{t}\left(t^{\prime}\right)\right| d t^{\prime},
\end{gathered}
$$

where the subscript 0 stands for normal and $t$ for tangential. At this point we can divide the contact models into:

- linear, e.g. Hooke,

- non-linear, e.g. Hertz.

The soft sphere approach is a well established method for granular material characterization (e.g., Aole et al. [6, Barrios et al. [13, Behera et al. [15, Frankowski et al. [46], Gremaud et al. [54, 55], Gustafsson et al. [61], Mio et al. [101], Pereira and Cleary [108, Pereira et al. [109, 110, 111], Torralba et al. [132]). 


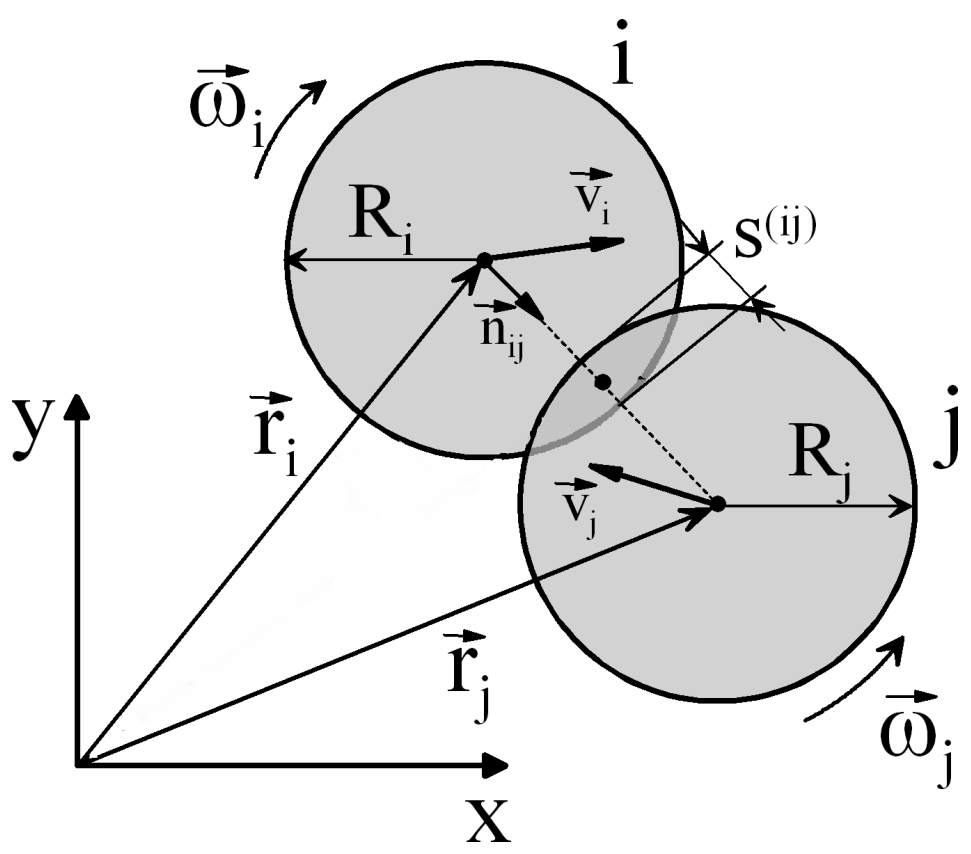

Figure 4.1: Two colliding spheres with the soft sphere approach [91]. The forces involved are shown.

\subsubsection{Hertz}

For the raw material used in this work Di Renzo and Di Maio [116] suggested using the non-linear Hertzian model without cohesion for the particle-particle and particle-wall contacts. For an elasto-perfectly-plasticviscous model the contact forces, normal and tangential, are:

$$
\begin{gathered}
\mathbf{F}_{n}=k_{n} \xi_{n} \mathbf{n}+\gamma_{n} \mathbf{v}_{n}, \\
\mathbf{F}_{t}= \begin{cases}k_{t} \xi_{t} \mathbf{t}+\gamma_{t} \mathbf{v}_{t} & \text { if }\left|k_{t} \xi_{t} \mathbf{t}+\gamma_{t} \mathbf{v}_{t}\right| \leq \mu_{s}\left|\mathbf{F}_{n}\right|, \\
\mu_{s}\left|\mathbf{F}_{n}\right| \mathbf{t} & \text { else. }\end{cases}
\end{gathered}
$$

The Coulomb's law of friction is the condition in the tangential force formulation.

Here, $k$ and $\gamma$ are respectively the stiffness and damping coefficients, while $\mathbf{v}$ is the velocity. Both the normal and the tangential force comprise two terms, a spring force and a damping force. The tangential (or shear) force is a "history" effect that accounts for the tangential displacement ("tangential overlap") between the particles for the duration of contact. The $k_{n}, k_{t}, \gamma_{n}$ and $\gamma_{t}$ coefficients are calculated from the material properties as follows:

$$
\begin{aligned}
k_{n} & =\frac{4}{3} E_{e q} \sqrt{R_{e q} \xi_{n}}, \\
\gamma_{n} & =2 \sqrt{\frac{5}{6}} \beta \sqrt{S_{n} m_{e q}} \\
k_{t} & =8 G_{e q} \sqrt{R_{e q}} \xi_{n}, \\
\gamma_{t} & =2 \sqrt{\frac{5}{6}} \beta \sqrt{S_{t} m_{e q}} .
\end{aligned}
$$


In addition to the equations 4.5 the following relations (Eqns. 4.6) are required:

$$
\begin{aligned}
\frac{1}{E_{e q}} & =\frac{1-\nu_{i}^{2}}{E_{i}}+\frac{1-\nu_{j}^{2}}{E_{j}} \\
\frac{1}{G_{e q}} & =\frac{2\left(2+\nu_{i}\right)\left(1-\nu_{i}\right)}{E_{i}}+\frac{2\left(2+\nu_{j}\right)\left(1-\nu_{j}\right)}{E_{j}} \\
\frac{1}{R_{e q}} & =\frac{1}{R_{i}}+\frac{1}{R_{j}} \\
\frac{1}{m_{e q}} & =\frac{1}{m_{i}}+\frac{1}{m_{j}} \\
\beta & =\frac{\ln (e)}{\sqrt{\ln n^{2}(e)+\pi^{2}}} \\
S_{n} & =2 E_{e q} \sqrt{R_{e q} \xi_{n}} \\
S_{t} & =8 G_{e q} \sqrt{R_{e q} \xi_{n}} \\
k_{r} & =k_{t} R_{e q}^{2} .
\end{aligned}
$$

The coefficient of sliding friction $\mu_{s}$ is one of the particle-based DEM parameter we investigated, another being the coefficient of rolling friction $\left(\underline{\mu_{r}}\right)$, which can be simulated with these models:

- constant direct torque (CDT),

- elasto-plastic-spring-dashpot.

In the papers by Harthong et al. [65], Hartl and Ooi [66], Iwashita and Oda [74, Wensrich and Katterfeld [139], further details on the methods can be found.

\subsubsection{Elasto-plastic-spring-dashpot 2 model}

For coarse non-spherical particles, $\mu_{r}$ is a critical parameter and describes inter-particle friction in medium to dense granular flow simulations. It is proportional to the torque counteracting the rotation of the particle. The $\mu_{r}$ parameter enters the equations according to the elasto-rolling resistance model presented by Ai et al. [1], Wensrich and Katterfeld [139] based on the work of Jiang et al. [76]. A scheme of the particles interaction can be seen in Fig. 4.2 .

The model is called EPSD2 in LIGGGHTS and is appropriate for both one-way and cyclical rolling

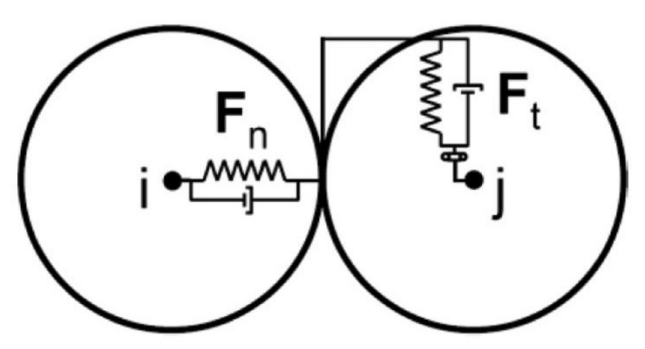

Figure 4.2: Spring dashpot model [91]. Newton's second law is applied in both directions, tangential and normal to the contact.

cases. The maximum magnitude of rolling resistance torque is (Eq. 4.7):

$$
T_{r, \max }=\mu_{r} R_{r}\left|F_{n}\right|
$$


where $R_{r}$ is the equivalent radius and $F_{n}$ the normal force. The last two particle-based DEM parameters we investigated were the particle density $\left(\overline{\rho_{p}}\right)$ and the coefficient of restitution $\left(C_{o R}\right)$ as defined by Ai et al. [1. Together with the others key parameters they are listed in Table 4.1

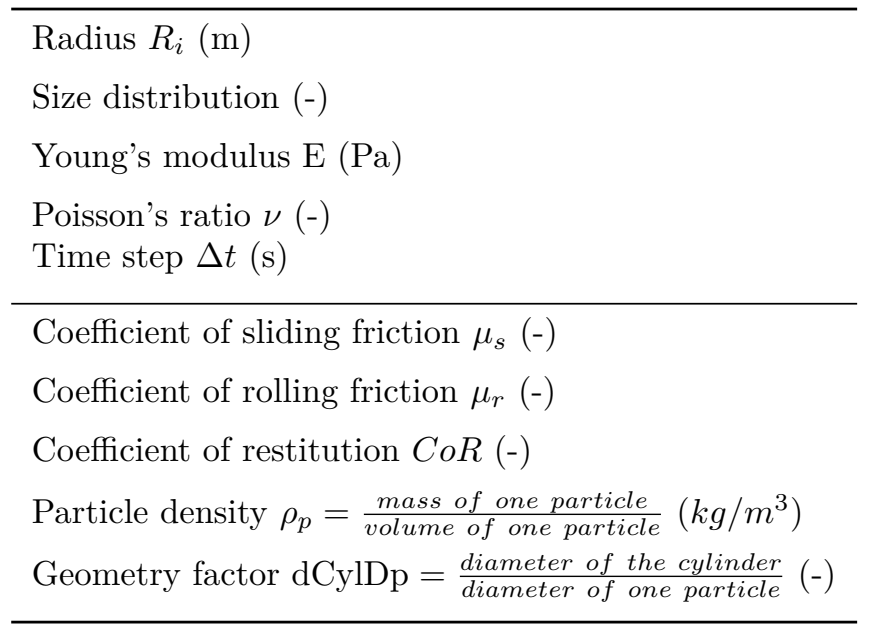

Table 4.1: DEM parameters. The upper parameters were identical in all simulations.

\subsection{Literature Values}

There are yet ways to determine contact parameters directly by measuring material properties or by performing particle based experiments ( [11, 21, 22, 27, 30, 31, 38, 40, 44, 49, 56, 58, 59, 64, 72, 73, 75, ,78. 80, 84, 90, 92, 98, 99, 102, 104, 107, 115, 127, 130, 131, 142]). Kruggel-Emden et al. 87, Langroudi et al. [88, Luding 93], M. et al. [96, Vidyapati [137] are some of the most prominent examples of parameters identification by theoretical analysis.

\subsubsection{Limitations}

The available literature data, however, focused on glass spheres, while non-sphericity was one of the fields we wanted to investigate. Further, it is related to all the microscopic parameters we analysed. Also the procedure of Combarros et al. 40, although on industrial samples (aluminium oxide), still had mostly spherical particles. In addition, these methodologies are laborious, since they have to be performed for every new granular material prior to a $D E M$ simulations. Especially for the already cited rolling friction parameter, it is arduous to link the rolling friction parameter to the non-sphericity of the particle.

\subsection{Unresolved CFD-DEM}

The existence of particles immersed in a fluid influences its behaviour. Keeping aside the short-scale flow field, the local averages inside the Navier-Stokes fluid equations could account for the particles. This is called the unresolved $C F D D E M$ approach, and can be seen in Fig. 4.3

On the hypothesis of having $g(r)$ a convenient averaging function, which is non-negative, smooth, asymptotically zero for large $r$, normalized, Lichtenegger [91] defines the local void fraction $(\varepsilon(\mathbf{r}, t))$, the volume of the fluid $\left(V_{f}(t)\right)$ over the total volume, as:

$$
\varepsilon(\mathbf{r}, t)=\int_{V_{f}(t)} g\left(\left|\mathbf{r}-\mathbf{r}^{\prime}\right|\right) \mathrm{d}^{3} r^{\prime},
$$




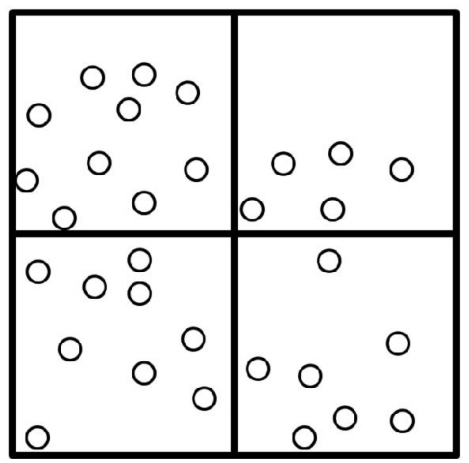

Figure 4.3: Unresolved CFD-DEM [62]. For systems like ours, where particles are considerably smaller than cell sizes, unresolved CFD-DEM is preferable to resolved CFD-DEM.

where $\mathbf{r}$ is the vector containing the position of a single particle and $\mathbf{r}^{\prime}$ the position of the its closest neighbour particle. We define local mean values of fluid point properties as:

$$
\bar{a}(\mathbf{r}, t)=\frac{1}{\varepsilon(\mathbf{r}, t)} \int_{V_{f}(t)} g\left(\left|\mathbf{r}-\mathbf{r}^{\prime}\right|\right) a\left(\mathbf{r}^{\prime}, t\right) \mathrm{d}^{3} r^{\prime},
$$

which came from a slowly varying a rapidly fluctuating part:

$$
a(\mathbf{r}, t)=\bar{a}\left(\mathbf{r}^{\prime}, t\right)+a^{\prime}(\mathbf{r}, t) .
$$

Particle properties $(p)$, on the solid volume $V_{s}(t)$ and the solid fraction $\phi(\mathbf{r}, t)$, are expressed as field quantities as:

$$
\begin{aligned}
\bar{b}(\mathbf{r}, t) & =\frac{1}{\phi(\mathbf{r}, t)} \int_{V_{f}(t)} g\left(\left|\mathbf{r}-\mathbf{r}^{\prime}\right|\right) b\left(\mathbf{r}^{\prime}, t\right) \mathrm{d}^{3} r^{\prime} \\
& \approx \frac{1}{\phi(\mathbf{r}, t)} \sum_{p} g\left(\mathbf{r}-\mathbf{r}_{p}\right) V_{p} b_{p}(t) .
\end{aligned}
$$

The approximation is valid when $g(r)$ varies slowly over the particles length scale and its surface $S_{p}$, and the averages of the derivatives can be written as:

$$
\begin{aligned}
\nabla_{\mathbf{r}} \varepsilon(\mathbf{r}, t) \bar{a}(\mathbf{r}, t) & =\int_{V_{f}(t)} a\left(\mathbf{r}^{\prime}, t\right) \nabla_{\mathbf{r}} g\left(\left|\mathbf{r}-\mathbf{r}^{\prime}\right|\right) \mathrm{d}^{3} r^{\prime} \\
& =-\int_{S_{f}(t)} a(\mathbf{S}, t) g(|\mathbf{r}-\mathbf{S}|) \mathrm{d} \mathbf{S}+\varepsilon(\mathbf{r}, t) \overline{\nabla a}(\mathbf{r}, t) \\
& \approx \sum_{p} \int_{S_{p}(t)} a(\mathbf{S}, t) g(|\mathbf{r}-\mathbf{S}|) \mathrm{d} \mathbf{S}+\varepsilon(\mathbf{r}, t) \overline{\nabla a}(\mathbf{r}, t) .
\end{aligned}
$$

Further, for time derivatives we can write:

$$
\begin{aligned}
\frac{\partial}{\partial t} \varepsilon(\mathbf{r}, t) \bar{a}(\mathbf{r}, t) & =\int_{V_{f}(t)} \frac{\partial a\left(\mathbf{r}^{\prime}, t\right)}{\partial t} g\left(\left|\mathbf{r}-\mathbf{r}^{\prime}\right|\right) \mathrm{d}^{3} r^{\prime} \\
& +\lim \frac{1}{\Delta t}\left[\int_{V_{f}(t+\Delta t)} a\left(\mathbf{r}^{\prime}, t\right) g(|\mathbf{r}-\mathbf{S}|) \mathrm{d}^{3} r^{\prime}-\int_{V_{f}(t)} a\left(\mathbf{r}^{\prime}, t\right) g(|\mathbf{r}-\mathbf{S}|) \mathrm{d}^{3} r^{\prime}\right] \\
& \approx \varepsilon(\mathbf{r}, t) \overline{\dot{a}}(\mathbf{r}, t)-\sum_{p} \int_{S_{p}(t)} \mathbf{v}_{\mathbf{s}} a(\mathbf{S}, t) g(|\mathbf{r}-\mathbf{S}|) \mathrm{d} \mathbf{S} .
\end{aligned}
$$




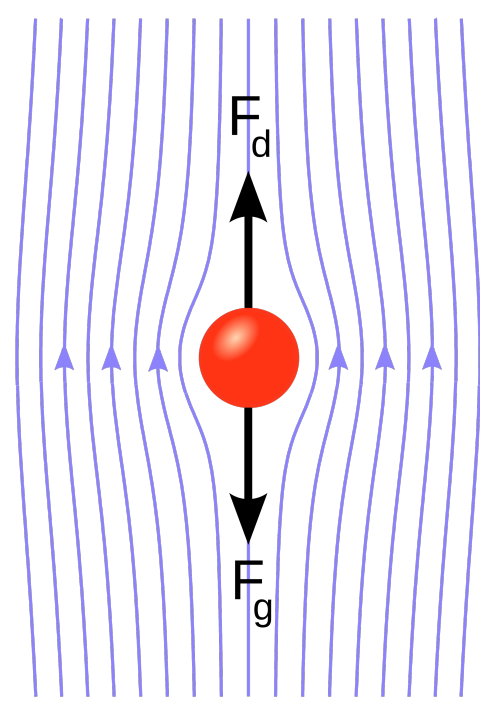

Figure 4.4: Particle in a fluid [14], with fluid flows, drag and gravity forces.

We could now write in Eq. 4.12 that $a=\rho \mathbf{u}$, respectively the density and the velocity of the fluid surronding the particle, as can be seen in Fig. 4.4 So, it becomes:

$$
\nabla_{\mathbf{r}} \varepsilon(\mathbf{r}, t) \overline{\rho \mathbf{u}}(\mathbf{r}, t) \approx \sum_{p} \int_{S_{p}(t)} \rho \mathbf{u}(\mathbf{S}, t) g(|\mathbf{r}-\mathbf{S}|) \mathrm{d} \mathbf{S}+\varepsilon(\mathbf{r}, t) \overline{\nabla \rho \mathbf{u}}(\mathbf{r}, t) .
$$

Similarly, for Eq. 4.13, we consider $a=\rho$, and we write:

$$
\frac{\partial}{\partial t}(\varepsilon(\mathbf{r}, t) \bar{\rho}(\mathbf{r}, t)) \approx \varepsilon(\mathbf{r}, t) \bar{\rho}(\mathbf{r}, t)-\sum_{p} \int_{S_{p}(t)} \mathbf{v}_{\mathbf{s}} \rho(\mathbf{S}, t) g(|\mathbf{r}-\mathbf{S}|) \mathrm{d} \mathbf{S} .
$$

At this point, it is easy to demonstrate that by merging Eq. 4.14 and Eq. 4.15 we obtain the volumeaveraged continuity equation:

$$
\frac{\partial}{\partial t}(\varepsilon(\mathbf{r}, t) \bar{\rho}(\mathbf{r}, t))+\nabla \cdot \varepsilon(\mathbf{r}, t) \bar{\rho} \mathbf{u}(\mathbf{r}, t)=0 .
$$

In case of incompressible flow $\frac{\partial \rho}{\partial t}=0$. Thus, Eq. 4.16 becomes:

$$
\frac{\partial}{\partial t} \varepsilon(\mathbf{r}, t)+\nabla \cdot \varepsilon(\mathbf{r}, t) \overline{\mathbf{u}}(\mathbf{r}, t)=0
$$

In the same way, for the Navier-Stokes momentum equation, including the stress tensor $\tau$ [63]:

$$
\int_{V_{f}(t)}\left[g\left(\left|\mathbf{r}-\mathbf{r}^{\prime}\right|\right) \rho_{f}\left(\frac{\partial \mathbf{u}}{\partial t}+(\mathbf{u} \cdot \nabla) \mathbf{u}\right)\right] \mathrm{d}^{3} r^{\prime}=\int_{V_{f}(t)}\left[g\left(\left|\mathbf{r}-\mathbf{r}^{\prime}\right|\right) \nabla \cdot \tau+\rho_{f} \mathbf{g}\right] \mathrm{d}^{3} r^{\prime},
$$

which can be written:

$$
\rho_{f} \varepsilon(\mathbf{r}, t)\left(\frac{\partial \overline{\mathbf{u}}}{\partial t}+(\overline{\mathbf{u}} \cdot \nabla) \overline{\mathbf{u}}\right)=\nabla \cdot(\bar{\tau}-\bar{R})-\sum_{p} g\left(\left|\mathbf{r}-\mathbf{r}_{\mathbf{p}}\right|\right) \mathbf{F}_{p-f}\left(\overline{\mathbf{u}}, \varepsilon, \mathbf{v}_{p}, d_{p}\right)+\rho_{f} \varepsilon(\mathbf{r}, t) \mathbf{g}
$$

with $\mathbf{F}_{p-f}$ as the interaction force between the particle and the fluid, or viceversa, considering Newton's third law. This force is dependant on the particle velocity $\mathbf{v}_{p}$ and the diameter of the particle $d_{p}$. Further:

$$
\bar{R}(\mathbf{r})=\rho_{f} \int_{V_{f}(t)} g\left(\left|\mathbf{r}-\mathbf{r}^{\prime}\right|\right) \mathbf{u}^{\prime}\left(\mathbf{r}^{\prime}, t\right) \circ \mathbf{u}^{\prime}\left(\mathbf{r}^{\prime}, t\right) \mathrm{d}^{3} r^{\prime} .
$$


To complete the system, we add the particles equation of motion:

$$
m_{p} \frac{\partial \mathbf{v}_{p}}{\partial t}=\mathbf{F}_{p-f}\left(\overline{\mathbf{u}}, \varepsilon, \mathbf{v}_{p}, d_{p}\right)+m_{p} \mathbf{g}+\Phi_{p-p}\left(\mathbf{r}_{p},\left\{\mathbf{r}_{k}\right\}\right),
$$

where $m_{p}$ is the mass of the particle, $\mathrm{g}$ the acceleration due to gravity, and $\Phi_{p-p}$ is a force field, which acts on particle $p$ because of the remaining particles. This unresolved $C F D D E M$ approach works as long as the exact trajectories of each particle can be negliged.

For completeness, we could say that also the equation of motion can be averaged over space, obtaining:

$$
\frac{\partial}{\partial t} \phi(\mathbf{r}, t) \rho_{p}(\mathbf{r}, t)+\nabla \cdot \phi(\mathbf{r}, t) \overline{\rho_{p} \mathbf{v}_{p}}(\mathbf{r}, t)=0 .
$$

If the spheres have the same density, we can write:

Finally:

$$
\frac{\partial}{\partial t} \phi(\mathbf{r}, t)+\nabla \cdot \phi(\mathbf{r}, t) \overline{\mathbf{v}_{p}}(\mathbf{r}, t)=0
$$

$$
\begin{aligned}
\rho_{p} \phi(\mathbf{r}, t)\left(\frac{\partial \overline{\mathbf{v}}_{p}}{\partial t}+\left(\overline{\mathbf{v}}_{p} \cdot \nabla\right) \overline{\mathbf{v}}_{p}\right) & =\sum_{p} g\left(\left|\mathbf{r}-\mathbf{r}_{\mathbf{p}}\right|\right) \Phi_{p-p}-\nabla \cdot S \\
& +\sum_{p} g\left(\left|\mathbf{r}-\mathbf{r}_{\mathbf{p}}\right|\right) \mathbf{F}_{p-f}+\phi(\mathbf{r}, t) \rho_{p} \mathbf{g}
\end{aligned}
$$

where:

$$
S(\mathbf{r})=\sum_{p} m_{p} g\left(\left|\mathbf{r}-\mathbf{r}_{p}\right|\right) \mathbf{v}_{p}^{\prime} \circ \mathbf{v}_{p}^{\prime}
$$

The main advantage of this model is to allow a greater number of particles to be considered, since they are treated as an additional averaged fluid: this is called the two-fluid model. Hovewer, this model work poorly in a dense system like our, see Hager [63], and was thus not considered further.

\subsubsection{Governing Equations}

From the equations derived in the previous paragraph, the software uses the following simplified governing equations 63., which can be divided into fluid and solid phase, given for both the same gravitational acceleration $g$. First, in the fluid phase the continuity equation can be written as:

$$
\frac{\partial \alpha_{f}}{\partial t}+\nabla \cdot\left(\alpha_{f} u\right)=0
$$

where $\alpha_{f}$ is the fluid fraction, and $u$ is the fluid velocity, while the momentum equation is:

$$
\frac{\partial \alpha_{f} \rho_{f} u}{\partial t}+\nabla \cdot\left(\alpha_{f} \rho_{f} u u\right)-\nabla \cdot\left(\alpha_{f} \tau\right)=-\alpha_{f} \nabla p+R_{s l}+\alpha_{f} \rho_{f} g .
$$

Here, $\rho_{f}$ is the fluid density, $\tau$ the shear stresses, $p$ the fluid pressure, and with $R_{s l}$, the force exchange term, given by:

$$
R_{s l}=\frac{1}{\Delta V} \sum f_{d, i}
$$

where $f_{d, i}$ are the drag forces for every particle in the computational cell, of which $\Delta V$ is the volume. Then, for the solid phase, we can write the equation of motion for each of the particles involved as:

$$
m_{p} \frac{\mathrm{d} u_{p}}{\mathrm{~d} t}=m_{p} g+f_{p, f}+\sum_{N_{p}} f_{p, p}+\sum_{N_{w}} f_{p, w},
$$

where $u_{p}$ is the velocity of the particle, $f_{p, p}$ the particle-particle interaction forces, while $f_{p, w}$ are the particle-wall interaction forces. The interaction force between the particle and the fluid is:

$$
f_{p, f}=f_{d}+f_{\nabla p}+f_{\nabla \tau}
$$

where $f_{d}$ is the drag force, $f_{\nabla p}$ the pressure gradient force, and $f_{\nabla \tau}$ the viscous force.

Closure of the system can be obtained with initial and boundary conditions for both phases. 


\subsection{Time Discretization}

According to the original article by Cundall and Strack [42, which defined first the DEM] it is necessary to computationally resolve the equations for particle contacts in discrete time steps, the length of which should be carefully considered. These time steps are small fractions of the total simulation time.

For pure DEM simulations, and for for the DEM portions of the coupled simulations, Cundall and Strack [42. suggest to fix a time step smaller than the $20 \%$ of the Rayleigh time, defined as:

$$
t_{r}=\pi r \cdot \frac{\sqrt{\frac{\rho_{p}}{G}}}{0.1631 \cdot \nu+0.8766},
$$

where $r$ is the radius of the smallest particle, $\rho_{p}$ its density, $G$ its shear modulus, and $\nu$ its Poisson ratio. For the CFD portions of the coupled simulations, Lichtenegger [91] states that the time step restrictions can be more easily complied. Thus, we used:

$$
\Delta t_{C F D}=10 \cdot \Delta t_{D E M}
$$

\subsection{Coupled Simulations}

The work of the unresolved CFD DEM software is illustrated in Fig. 4.5, and can be found in details in Lichtenegger [91. In our simulations, the whole diagram represents only one CFD step and 10 DEM steps. In fact, the Calculation of forces block should be read as 10 DEM time steps for the calculation of the particle forces. This is necessary because of the severe time discretization requirements inherent the method. 


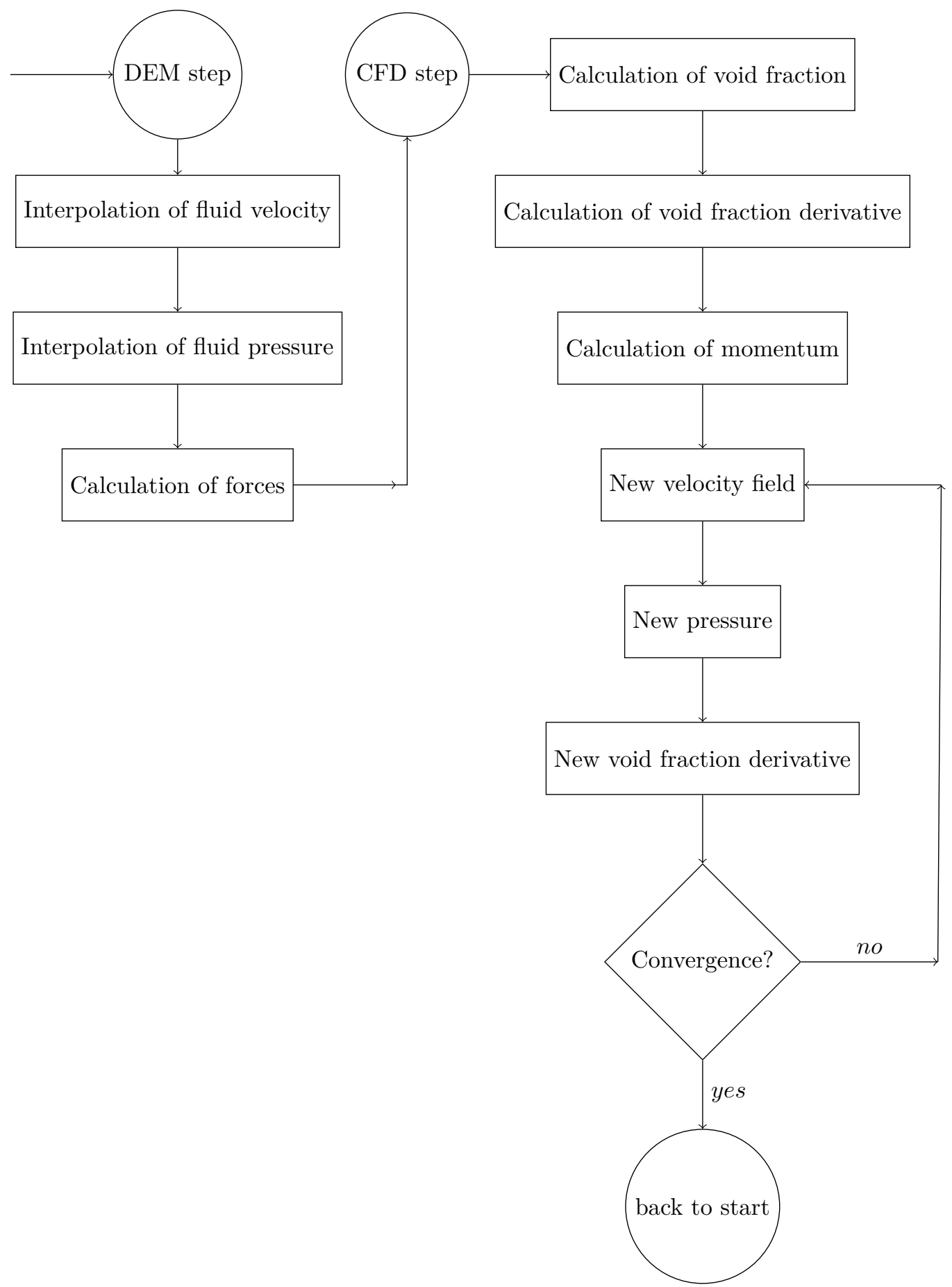

Figure 4.5: Computational CFD-DEM steps in a simulation. These steps are iterated many times until convergence and duration are both achieved. 


\section{Chapter 5}

\section{Artificial Neural Network}

\subsection{Probability theory}

Bishop 23 defines $X$ a random variable with a fixed domain $\operatorname{Val}(X)$, showing aspects of the system's world. In this world, a fixed assignment of values to variables (from one to all) is called an event. Given a probability distribution $P$, this assigns to the specific event $\alpha$ a value: $P(\alpha)$.

Provided a random variable $X$, whose $\operatorname{Val}(X)=\left\{x^{1}, \ldots, x^{k}\right\}$, a Probability Distribution over $X$ is $P(X)=\left\{P\left(x^{1}\right), \ldots, P\left(x^{k}\right)\right\}$.

He further defines, given a second random variable $Y$ :

- probability of conjunction of events, $P(X=x, Y=y)$ or $P(x, y)$, or $P((X=x) \cap(Y=y))$;

- a set of variables, $\mathbf{X}=\left\{X_{1}, X_{2}, \ldots, X_{k}\right\}$;

- joint distribution over one set of variables, $P(\mathbf{X})=P\left(X_{1}, X_{2}, \ldots, X_{k}\right)$;

- joint distribution over two sets of variables, $P(\mathbf{X}, \mathbf{Y})=P\left(X_{1}, X_{2}, \ldots, X_{k}, Y_{1}, Y_{2}, \ldots, Y_{l}\right)$;

- conditional distribution, $P(\mathbf{X} \mid \mathbf{Y})=P\left(X_{1}, X_{2}, \ldots, X_{k} \mid Y_{1}, Y_{2}, \ldots, Y_{l}\right)$.

For later reference, we write two fundamental rules of the probability theory, the sum rule (Eq. 5.1) and the product rule (Eq. 5.2), from which Bayes derived his theorem (Eq. 5.3):

$$
\begin{gathered}
P(X)=\sum_{Y} P(X, Y), \\
P(X, Y)=P(Y \mid X) \cdot P(X), \\
P(Y \mid X)=\frac{P(X \mid Y) \cdot P(Y)}{P(X)} .
\end{gathered}
$$

\subsubsection{The Gaussian Distribution}

Provided a single real valued variable $x$, a Gaussian distribution is:

$$
\mathcal{N}\left(x \mid \mu, \sigma^{2}\right)=\frac{1}{\left(2 \pi \sigma^{2}\right)^{0.5}} \exp \left\{-\frac{1}{2 \sigma^{2}}(x-\mu)^{2}\right\},
$$

where $\mu$ is the mean and $\sigma^{2}$ is the variance, of which the square root is the standard deviation. Further, $\beta=1 / \sigma^{2}$ is the deviation. Given a set of observations $\mathbf{x}=\left(x_{1}, \ldots, x_{N}\right)^{T}$, assumed indipendent and identically distributed (i.i.d.), the probability of the data set becomes:

$$
p\left(\mathbf{x} \mid \mu, \sigma^{2}\right)=\prod_{n=1}^{N} \mathcal{N}\left(x \mid \mu, \sigma^{2}\right)
$$


which can be more easily computed if written in a logaritmic form:

$$
\ln p\left(\mathbf{x} \mid \mu, \sigma^{2}\right)=-\frac{1}{2 \sigma^{2}} \sum_{n=1}^{N}\left(x_{n}-\mu\right)^{2}-\frac{N}{2} \ln \left(\sigma^{2}\right)-\frac{N}{2} \ln (2 \pi) .
$$

To obtain the expectation of this likelihood Equation 5.6 can be maximized over $\mu$ and $\sigma^{2}$, giving respectively Eq. 5.7 and Eq. 5.8

$$
\begin{gathered}
\mathbb{E}\left[\mu_{M L}\right]=\mathbb{E}\left[\frac{1}{N} \sum_{n=1}^{N}\left(x_{n}\right)\right]=\mu, \\
\mathbb{E}\left[\sigma_{M L}^{2}\right]=\mathbb{E}\left[\frac{1}{N} \sum_{n=1}^{N}\left(x_{n}-\mu_{M L}\right)^{2}\right]=\left(\frac{N-1}{N}\right) \sigma^{2} .
\end{gathered}
$$

In case of a D-dimensional vector $\mathbf{x}$, we write the multivariate Gaussian distribution as:

$$
\mathcal{N}(\mathbf{x} \mid \boldsymbol{\mu}, \boldsymbol{\Sigma})=\frac{1}{(2 \pi)^{D / 2}|\boldsymbol{\Sigma}|^{0.5}} \exp \left\{-\frac{1}{2}(\mathbf{x}-\boldsymbol{\mu})^{T} \boldsymbol{\Sigma}^{-1}(\mathbf{x}-\boldsymbol{\mu})\right\}
$$

where $\boldsymbol{\Sigma}$ is the covariance matrix.

\subsection{Principal Component Analysis (PCA)}

One of the most used statistical tecniques is the Principal Component Analysis (PCA). Bishop [23] defines it as a linear projection, which minimizes the average projection cost. This cost corresponds to the mean squared distance between the data points investigated and their projections. In our case we aimed to:

- investigate the linear relationships between contact law parameters and bulk values;

- ascertain the necessity for more precise statistical tools, i.e. regression.

\subsection{Regression statistics}

Statistics finds relationships between random variables. Once one of them (response) is chosen, it infers its dependance from the others (regressors), also including an additive error term (Bishop [23, Brown and Nelson [29], Christopoulos and Lew [36], Costa et al. [41]). We call this a regression model, which can be linear or nonlinear. In this work we exploited the following regression models:

- Bayesian linear,

- Gaussian non linear,

- Artificial Neural Network (ANN).

We later explained, Section 8.3 , why we focused on the last one over the other two.

\subsubsection{Learning processes}

The learning processes available for a regression model are:

- unsupervised learning,

- semi-supervised learning, or reinforcement learning, 
- supervised learning, or learning with a teacher.

Unsupervised learning does not count on the presence of a teacher. It focuses on a task-indipendent measure of the quality of the representation, which the network must learn. This learning model is required to optimize its parameters confronting this measure.

A further step is semi-supervised learning, where an online interaction between the network and its environment provides the training.

\subsubsection{Supervised learning}

The easiest regression model consists in fitting target data points (exptected response) with a polynomial curve. The cofficients of the curve are obtained by minimizing a cost function, which is proportional to the distance of said data points to the curve. The expected response represents the teacher of the system. The network is trained in batch mode, all the examples are known before starting the training. The examples represent the teacher of the system. Further, these examples are assumed to be independent and identically distributed. We focused on supervised learning: the materials we investigated were examined in batch, not online.

Bishop [23] defines supervised learning an application, where all the training examples comprises the input vectors and their corresponding target vectors.

\subsubsection{The Standard Bayesian Linear Model}

Boccignone [25], Rasmussen and Nickisch [114] define the standard linear regression model with Gaussian noise as:

$$
\begin{aligned}
y(\mathbf{x}, \mathbf{w}) & =w_{0}+w_{1} x_{1}+\ldots+w_{n} x_{n} \\
& =w_{0}+\sum_{j=1}^{M-1} w_{j} \mathbf{x}_{\mathbf{j}} \\
& =\sum_{j=0}^{M-1} w_{j} \mathbf{x}_{\mathbf{j}} \\
& =\mathbf{w}^{T} \mathbf{x} .
\end{aligned}
$$

where $\mathbf{x}$ is the input vector of the regressors, $\mathbf{w}$ are the weights of the linear model, and $y$ is the target value, the response. The noise $\varepsilon$ adopts an i.i.d. Gaussian distribution:

$$
\varepsilon=\mathcal{N}\left(0, \sigma_{n}^{2}\right) .
$$

Extending to the n-dimensional case, we have a set of $n$ observations:

$$
\mathcal{D}=\left\{\left(\mathbf{x}_{i}, y_{i}\right) \mid i=1, \ldots, n\right\}=(X, \mathbf{y}) .
$$

Consequently, the likelihood, or probability density of the observations given the parameters, becomes:

$$
\begin{aligned}
p(\mathbf{y} \mid X, \mathbf{w}) & =\prod_{i=1}^{n} p\left(y_{i} \mid \mathbf{x}_{i}, \mathbf{w}\right)=\prod_{i=1}^{n} \frac{1}{\sqrt{2 \pi} \sigma_{n}} \exp \left(-\frac{\left(y_{i}-\mathbf{x}_{i}^{T} \mathbf{w}\right)^{2}}{2 \sigma_{n}^{2}}\right) \\
& =\frac{1}{\left(2 \pi \sigma_{n}^{2}\right)^{n / 2}} \exp \left(-\frac{1}{2 \sigma_{n}^{2}}\left|\mathbf{y}-X^{T} \mathbf{w}\right|^{2}\right)=\mathcal{N}\left(X^{T} \mathbf{w}, \sigma_{n}^{2} I\right),
\end{aligned}
$$

where the Euclidean length of vector $\mathbf{v}$ is indicated by $|\mathbf{v}|$. We also consider the weights $\mathbf{w}$ proportional to the Gaussian distribution with zero mean and variance equal to the coviarance matrix $\Sigma_{p}$. Remembering the theorem of Bayes, Eq. 5.3, which states that:

$$
\text { posterior }=\frac{\text { likelihood } \times \text { prior }}{\text { marginal likelihood }},
$$


we can define the continuous posterior distribution as:

$$
p(\mathbf{w} \mid \mathbf{y}, X)=\frac{p(\mathbf{y} \mid X, \mathbf{w}) \cdot p(\mathbf{w})}{p(\mathbf{y} \mid X)},
$$

where the marginal likelihood is:

$$
p(\mathbf{y} \mid X)=\int p(\mathbf{y} \mid X, \mathbf{w} p(\mathbf{w}) d \mathbf{w} .
$$

A first estimation of the posterior can be determined as follow:

$$
\begin{aligned}
p(\mathbf{w} \mid X, \mathbf{y}) & \propto \exp \left(-\frac{1}{2 \sigma_{n}^{2}}\left(\mathbf{y}-X^{T} \mathbf{w}\right)^{T}\left(\mathbf{y}-X^{T} \mathbf{w}\right)\right) \exp \left(-\frac{1}{2} \mathbf{w}^{T} \Sigma_{p}^{-1} \mathbf{w}\right) \\
& \propto \exp \left(-\frac{1}{2}(\mathbf{w}-\overline{\mathbf{w}})^{T}\left(\frac{1}{\sigma_{n}^{2}} X X^{T}+\Sigma_{p}^{-1}\right)(\mathbf{w}-\overline{\mathbf{w}})\right) \\
& \sim \mathcal{N}\left(\overline{\mathbf{w}}=\frac{1}{\sigma_{n}^{2}} A^{-1} X \mathbf{y}, A^{-1}\right),
\end{aligned}
$$

where $A=\sigma_{n}^{-2} X X^{T}+\Sigma_{p}^{-1}$.

An effective tool for the posterior predictive density of a variational Bayesian multiple linear regression model is provided by Brodersen et al. 28.

\subsubsection{Gaussian Process}

Rasmussen and Nickisch [114] define a Gaussian process as a set of random variables. Any finite random of these variables have a joint Gaussian distribution. The process $f(\mathbf{x})$ can be defined by its mean function $m(\mathbf{x})$ and its covariance function $k\left(\mathbf{x}, \mathbf{x}^{\prime}\right)$, which are, respectively:

$$
\begin{gathered}
m(\mathbf{x})=\mathbb{E}[f(\mathbf{x})], \\
k\left(\mathbf{x}, \mathbf{x}^{\prime}\right)=\mathbb{E}\left[(f(\mathbf{x})-m(\mathbf{x}))\left(f\left(\mathbf{x}^{\prime}\right)-m\left(\mathbf{x}^{\prime}\right)\right)\right],
\end{gathered}
$$

and it can be written in a compact form as:

$$
f(\mathbf{x}) \sim \mathcal{G P}\left(m(\mathbf{x}), k\left(\mathbf{x}, \mathbf{x}^{\prime}\right)\right)
$$

In fact, the Bayesian linear regression model is a subcase of the Gaussian process model, with the following conditions:

$$
\begin{aligned}
f(\mathbf{x}) & =\phi(\mathbf{x})^{T} \mathbf{w}, \\
\mathbf{w} & \sim \mathcal{N}\left(\mathbf{0}, \Sigma_{p}\right), \\
\mathbb{E}[f(\mathbf{x})] & =\phi(\mathbf{x})^{T} \mathbb{E}[\mathbf{w}]=0, \\
\mathbb{E}\left[f(\mathbf{x}) f\left(\mathbf{x}^{\prime}\right)\right] & =\phi(\mathbf{x})^{T} \mathbb{E}\left[\mathbf{w} \mathbf{w}^{T}\right] \phi\left(\mathbf{x}^{\prime}\right)=\phi(\mathbf{x})^{T} \Sigma_{p} \phi\left(\mathbf{x}^{\prime}\right) .
\end{aligned}
$$

We used the squared exponential (SE), also called Radial Basis Function (RBF) or Gaussian Function, as covariance function. It defines the covariance between a pair of random variables as:

$$
\operatorname{cov}\left(f\left(\mathbf{x}_{p}\right), f\left(\mathbf{x}_{q}\right)\right)=k\left(\mathbf{x}_{p}, \mathbf{x}_{q}\right)=\exp \left(-\frac{1}{2}\left|\mathbf{x}_{p}-\mathbf{x}_{q}\right|^{2}\right) .
$$

Specifically, we handled the software developed by Rasmussen and Nickisch [114 for Gaussian processes inference and prediction, although we constrained ourselves to the case of exact inference (regression with Gaussian likelihood). The complete description of the Gaussian non linear regression can be found in Bishop [23]. 


\subsection{Introduction on Artificial Neural Networks}

An Artificial Neural Network (ANN) is a powerful regression model, based on non-linear functions (Gurney [60], Haykin [67]). In fact, we can see a neural network as a huge and parallel computer, composed of many processing units. It stores experienced knowledge and translates it in an usable form. The biological inspiration for artificial neural networks can be seen in Fig. 5.1. It resembles the human brain especially because it uses a learning process to acquire knowledge, which is stored through the synaptic weights.

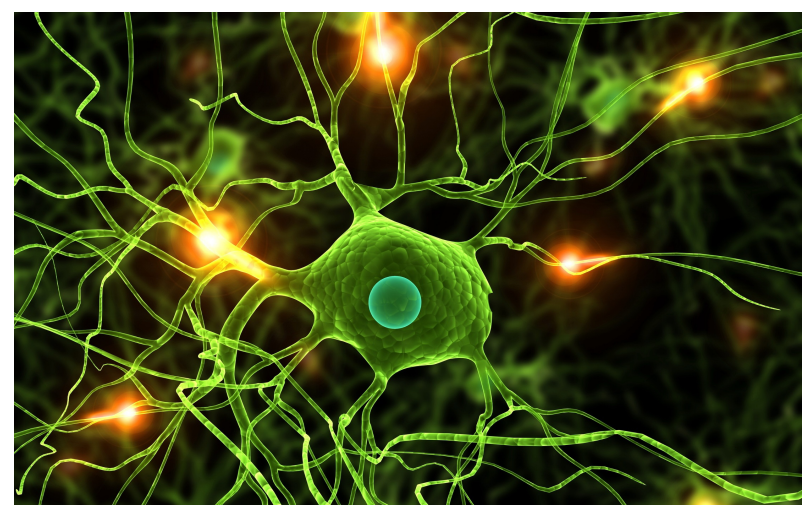

Figure 5.1: Biological inspiration 2 for the Artificial Neural Networks: a human neuron with the incoming electric signals [67].

In this work, we first used the ANN to fit the DEM numerical simulation data, and then to process a vast number of parameters combinations. ANNs map combinations of input data to convenient outputs (fitting). Amongst the available ANNs were important for our context:

- the Feedforward (FF) $\mathrm{ANN}$

- the Radial basis function (RBF) ANN

We did not investigate the latter, since its algorithm is a sub-case of the Gaussian Process we already implemented. For FF ANN numerous training algorithms are available. The most common are based on backpropagation, e.g., Levenberg-Marquardt, Bayesian regulation and the scaled conjugate gradient.

\subsection{Perceptron}

The basic element of an artificial neural network is the perceptron, a single neuron, see Fig. 5.2. It is composed by inputs $\left(x_{1}, x_{2}, \ldots, x_{m}\right)$, weights for these inputs $\left(w_{1}, w_{2}, \ldots, w_{m}\right)$ and a bias $b$. A linear combination of these elements, and the incorporation of the bias, forms the induced local field [67]:

$$
v=b+\sum_{j=1}^{m} w_{j} \cdot x_{j} .
$$

Then, we can obtain the output $y$ thanks to the activation function $\varphi(\cdot)$ :

$$
y=\varphi(v) .
$$

In the simplest case the activation function $\varphi(\cdot)$ is a signum function, and divides the inputs in two classes. Consequently, $y$ can be +1 , for positive inputs, or -1 , for negative inputs. Inputs of the first class belong to class $\mathscr{C}_{1}$, of the second to class $\mathscr{C}_{2}$. Thus, the patterns $(y)$ are hypothesised to be linearly separable. In this case the training, as identification of the correct weights and bias, can be precisely mathematically described. 


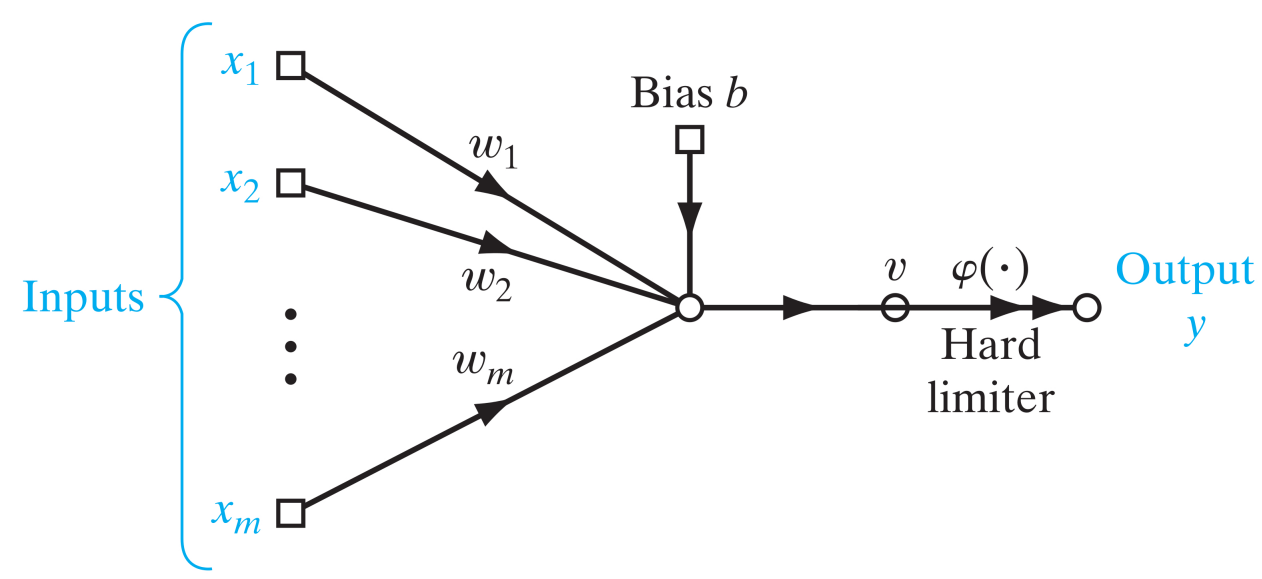

Figure 5.2: Perceptron scheme [67]. There can be many regressors, but only one response.

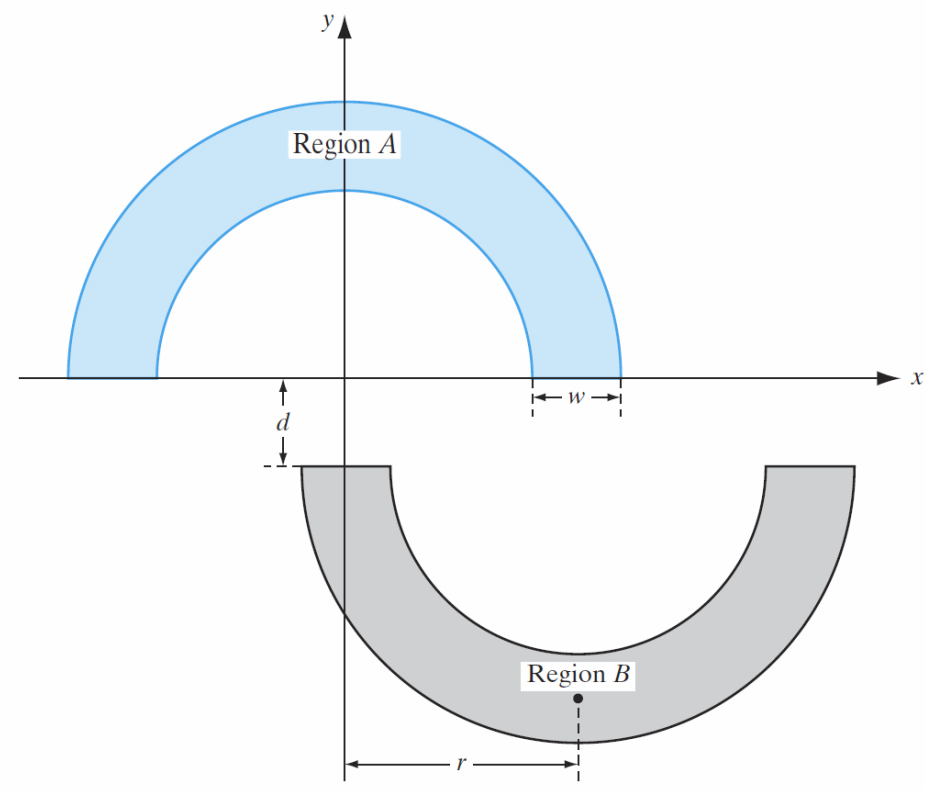

Figure 5.3: Double moon scheme [67]. Literature challenge to distinguish between linear and non linear separation algorithms.

\subsubsection{Double Moon}

The classical example of the double moon, see Fig. 5.3. clarifies the limits of the single perceptron approach, especially why a single neuron is not capable to handle a nonlinear problem. We drew two moons, both with an inner radius $r$. Their minimum distance is $d$. We could consider the two moons as composed by a finite number of two-dimensional points. We could use a portion of these points to train the perceptron, i.e. to teach the network which points belong to a region and which belong to the other. Goal of the perceptron is to divide the two-dimensional into two separate regions. As long as $d$ is positive, the investigation space can easily be linearly separated in two regions. Once trained, the perceptron can assign the remaining points to one moon or the other according to the training. 


\subsection{Multilayer}

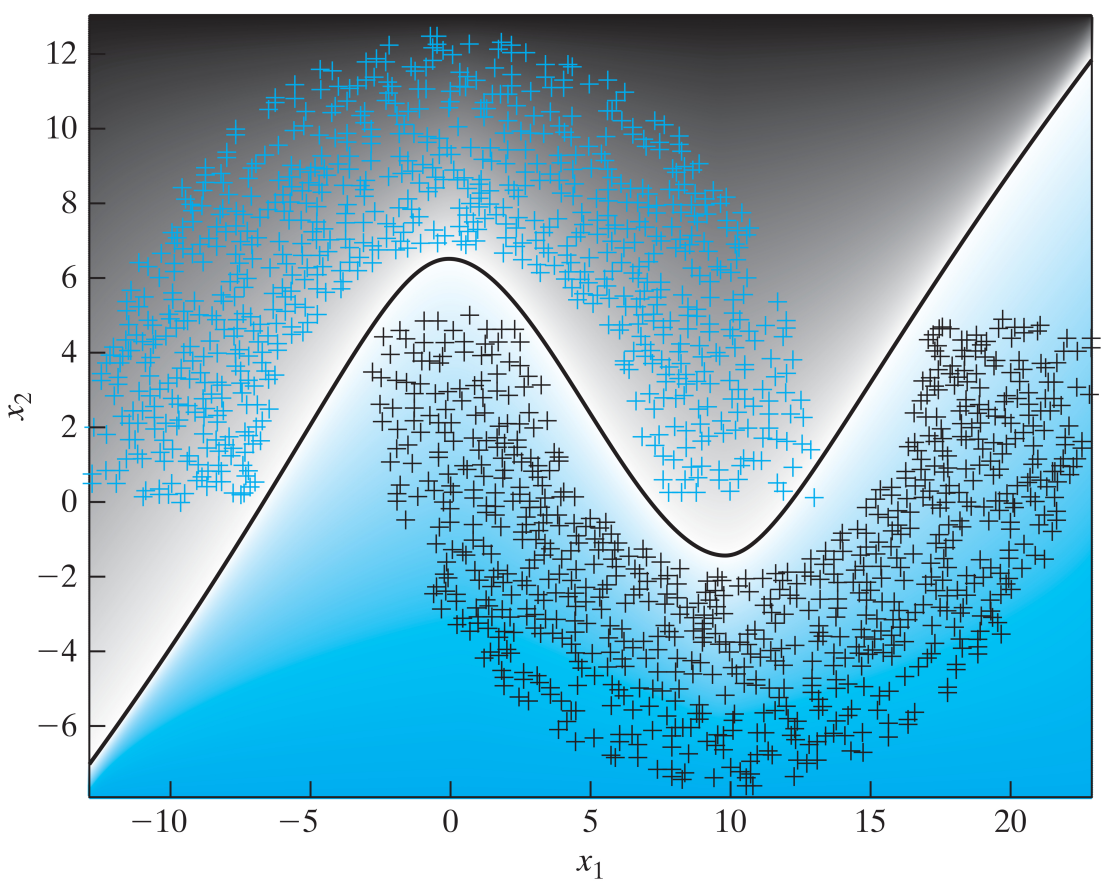

Figure 5.4: Double moon classification problem [67]. Literature challenge to distinguish between linear and non linear separation algorithms.

The handling of multiple outputs or, in our example, when $d$ becomes negative, see Fig. 5.4 makes linear separation not possible, and a multineuron approach necessary. Many neurons are combined to

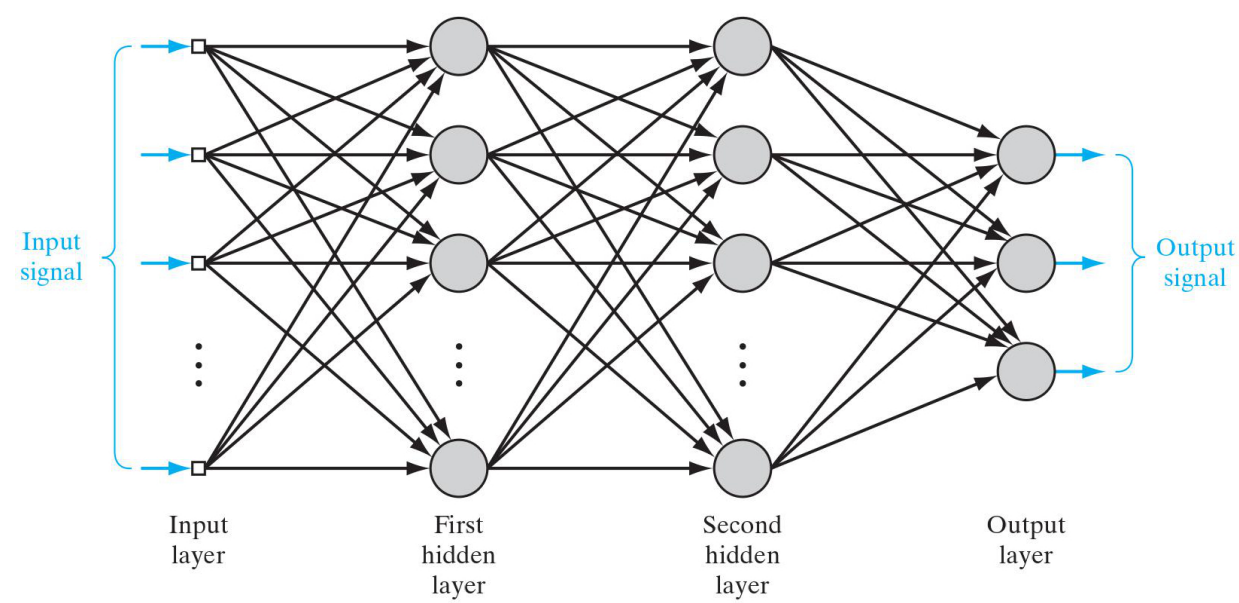

Figure 5.5: Multilayer perceptron architecture, with emphasys over the componing layers [67].

form a network, as in the architecture presented in Fig. 5.5. With more neurons, we can obtain a nonlinear solution of our separation problem (a nonlinear function, the black continuous line), and correctly classify the input, as shown in Fig. 5.4.

The first requirement of a multilayer neural network is that each neuron of the network must have dif- 
ferentiable nonlinear activation function, such as the strictly increasing hyperbolic tangent. Further, the network must have at least one layer hidden from both the input and the output nodes, see Fig. 8.2 to compute higher-order statistics.

An high degree of connectivity is another salient characteristic, dependent on the synaptic weights of the network. Usually, each neuron of one layer is linked with all the neurons in the previous layer. A network is called feedforward $\mathrm{FF}$ if the inputs of the neurons in one layer are exclusively the output signals of the neurons in the previous layer.

\subsection{Batch Learning}

A Multilayer ANN can be trained in a supervised fashion with a training sample

$$
\mathscr{T}=\{\mathbf{x}(n), \mathbf{d}(n)\}_{n=1}^{N},
$$

where $\mathbf{x}(n)$ is the stimulus applied to the input layer. The error is calculated as the difference at the $\mathrm{j}$-th node between the output of the neuron $\left(y_{j}(n)\right)$ and the expected response $\left(d_{j}(n)\right)$ :

$$
e_{j}(n)=d_{j}(n)-y_{j}(n)
$$

Thus, $\mathbf{d}(n)$ is the desired response vector, and $d_{j}(n)$ is its j-th element. As in the Least Mean Square algorithm (see Haykin [67]), we define the instanteneous error energy for the $\mathrm{j}$-th neuron as:

$$
\mathscr{E}_{j}(n)=\frac{1}{2} e_{j}^{2}(n)
$$

If we sum over all the neurons in the output layer, we obtain the total instanteneous error energy as:

$$
\begin{aligned}
\mathscr{E}(n) & =\sum_{j \in C} \mathscr{E}_{j}(n) \\
& =\frac{1}{2} \sum_{j \in C} e_{j}^{2}(n)
\end{aligned}
$$

One epoch of training consists in the calibration of the synaptic weights after the presentation of all the $N$ training samples in $\mathscr{T}$ Thus, the cost function $[\mathscr{E}(\mathbf{w})$ to be minimized can be expressed as the error energy averaged over the training sample:

$$
\begin{aligned}
\mathscr{E}_{a v}(N) & =\frac{1}{N} \sum_{n=1}^{N} \mathscr{E}(n) \\
& =\frac{1}{2 N} \sum_{n=1}^{N} \sum_{j \in C} e_{j}^{2}(n) .
\end{aligned}
$$

In the following epoch, the training sample $\mathscr{T}$ is randomly shuffled, and the cost function is once more computed. We define the gradient vector $\mathbf{g}(n)$ as:

$$
\mathbf{g}(n)=\left.\frac{\partial \mathscr{E}_{a v}(\mathbf{w})}{\partial \mathbf{w}}\right|_{\mathbf{w}=\mathbf{w}(n)},
$$

or the derivative of the cost function over the weight vector. The batch learning method grants an accurate prediction of the gradient vector, and consequently, given defined prerequisites, the convergence to a local minimum of the method of the steepest descent.

Finally, the curvature of the error performance surface is the Hessian matrix, written as:

$$
\mathbf{H}(n)=\left.\frac{\partial^{2} \mathscr{E}_{a v}(\mathbf{w})}{\partial \mathbf{w}^{2}}\right|_{\mathbf{w}=\mathbf{w}(n)}
$$




\subsection{Backpropagation algorithm}

Haykin [67] defines the induced local field for a multilayer ANN as:

$$
v_{j}(n)=\sum_{i=0}^{m} w_{j} i(n) \cdot y_{i}(n),
$$

for the $\mathrm{j}$-th neuron and the $\mathrm{n}$-th example, given $m$ inputs. The case of $i$ equal to zero refers to the bias. Consequently, the function signal $y_{j}(n)$ for neuron $j$ becomes:

$$
y_{j}(n)=\varphi\left(v_{j}(n)\right) \text {. }
$$

Thus, the weight $w_{j i}(n)$ of the $i$-th input for the $j$-th neuron with the $n$-th example is corrected by the back-propagation algorithm as follow:

$$
\Delta w_{j i}(n)=-\eta \frac{\partial \mathscr{E}(n)}{\partial w_{j i}(n)},
$$

where the fraction represents the sensitivity factor and $\eta$ is the learning-rate parameter, which could be a user tuned parameter. We can also write it as proportional to the local gradient $\delta_{j}(n)$ :

$$
\Delta w_{j i}(n)=-\eta \delta_{j}(n) y_{i}(n) .
$$

A multilayer perceptron network can be trained with the supervised back-propagation algorithm, composed of two phases, forward and backward, as shown in Fig. 5.6. In the first phase the weights do not

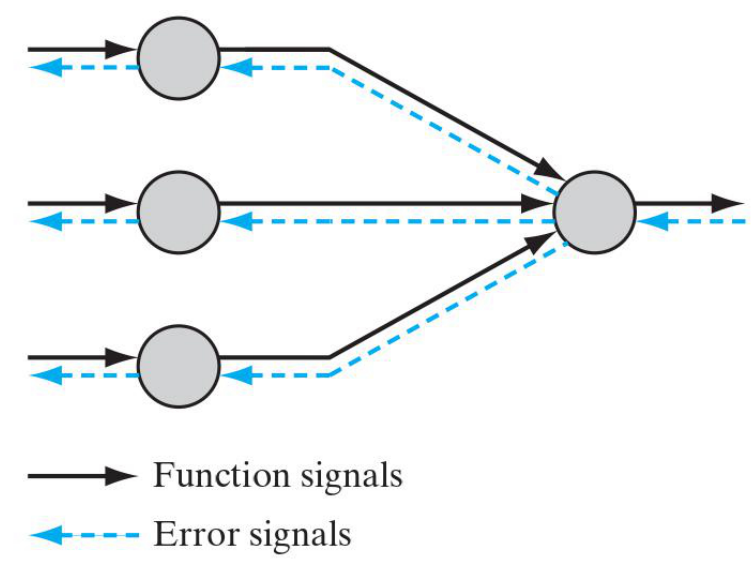

Figure 5.6: FF Backpropagation [67]. Direction of the learning is shown by the arrows.

change. From the inputs through the hidden and output layers the output is computed. The backward pass moves in the opposite direction, from right to left. The local gradient $\delta$ is computed for each neuron, layer by layer. Thus, the weights can be tuned accordingly to the delta rule of Eq. 5.35 .

The variations of the weights in each loop can be more or less consistent, accordingly to the learning-rate parameter $\eta$. A large $\eta$ makes the algorithm fast, while a small one prevents divergence. Converged is reached when the absolute rate of change in the average squared error per epoch is sufficiently small [67]. In our case, when $\Delta w<10^{-4}$. 


\subsubsection{Backpropagation efficacy}

The validity of the FF Multilayer Perceptron Neural Networks (MLPNN), with a backpropagation reinforcement learning training algorithm, has been demonstrated in the literature, see Haykin [67]. Several scientists [5, 12, 32, 43, 89, 125, 134, 136, have employed ANNs to model the mechanical properties of materials.

\subsection{Optimization}

The quadratic approximation of the backpropagation algorithm can be performed with the following methods:

- conjugate gradient,

- Newton and quasi-Newton,

- Levenberg-Marquardt.

Compared to a linear approximation the conjugate gradient method is more precise. Further, it is faster and less computationally demanding than the remaining quadratic methods.

\subsubsection{Conjugate Gradient Algorithm}

First, the weight vector $\mathbf{w}(0)$ is randomly initialized. Haykin [67] defines residual as the steepest-descent direction:

$$
\mathbf{r}(n)=\mathbf{g}(n)-\mathbf{H}(n) \mathbf{w}(n) .
$$

Further, the conjugate gradient vectors $\mathbf{s}(n)$, which name the method, are:

$$
\mathbf{s}(n)=\mathbf{r}(n)+\beta(n) \mathbf{s}(n-1),
$$

where $\beta(n)$ is calculated with the Fletcher - Reeves formula as:

$$
\beta(n)=\frac{\mathbf{r}^{T}(n) \mathbf{r}(n)}{\mathbf{r}^{T}(n-1) \mathbf{r}(n-1)} .
$$

Consequently, the algorithm is composed by the following steps:

1. From $\mathbf{w}(0)$, we calculate $\mathbf{g}(0)$, the gradient vector, through backpropagation.

2. Fix $\mathbf{s}(0)=\mathbf{r}(0)=-\mathbf{g}(0)$.

3. To find the $\eta(n)$, which maximize the $\mathscr{E}_{a v}(\eta)$, compute a line search at the time step $n$.

4. Check the Euclidean norm of the residual $\Delta w$. If it is under a small fraction of the initial number, then the method stops.

5. The weight vector is updated:

$$
\mathbf{w}(n+1)=\mathbf{w}(n)+\eta(n) \mathbf{s}(n) .
$$

6. The gradient vector $\mathbf{g}(n+1)$ is updated through back propagation for $\mathbf{w}(n+1)$.

7. Fix $\mathbf{r}(n+1)=-\mathbf{g}(n+1)$.

8. Through the Fletcher - Reeves formula compute $\beta$ :

$$
\beta(n+1)=\max \left\{\frac{\mathbf{r}^{T}(n+1) \mathbf{r}(n+1)}{\mathbf{r}^{T}(n) \mathbf{r}(n)}, 0\right\} .
$$


9. The direction vector is updated:

$$
\mathbf{s}(n+1)=\mathbf{r}(n+1)+\beta(n+1) \mathbf{s}(n) .
$$

10. Fix $n=n+1$, and start again from step 3 .

The gradient methods stops when the residuals at the n-th step are a small fraction of their initial values.

\subsection{Generalization}

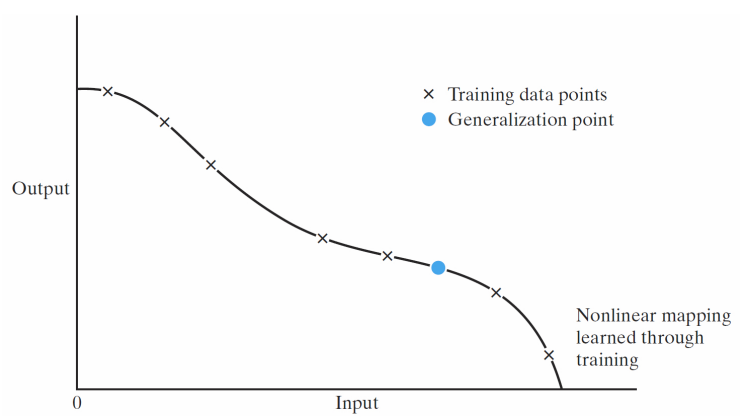

(a) Good fitting.

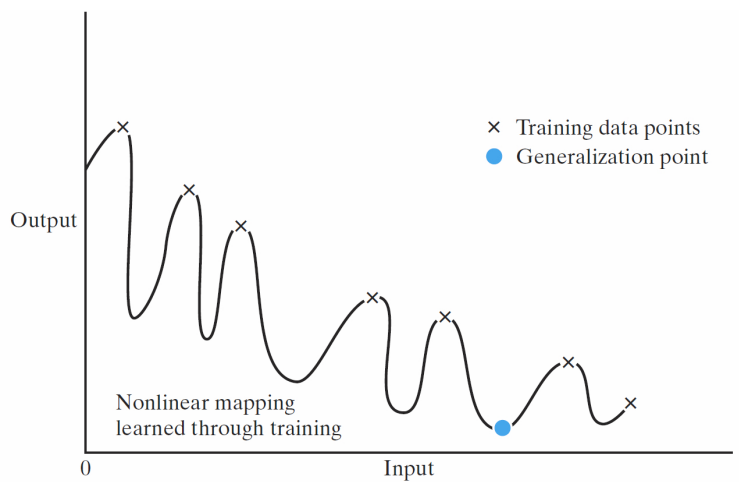

(b) Bad fitting.

Figure 5.7: Fitting, or agreement, between data point and regression, or interpolation, functions [67].

Haykin [67] suggests to carefully analyze two extreme situations:

- underfitting (or undertraining),

- overfitting (or overtraining),

when training a network.

Correctly computed input-output mapping is a synonym of a well generalized network, and of good nonlinear interpolation of the inputs, as in Fig. 5.7a. On one hand, underfitting means that there were not enough neurons to characterize the given function.

On the other hand, an excessive amount of neurons, known as overfitting, leads to poor generalization, see Fig. 5.7b. Also the architecture of the neural network and physical complexity of the problem influence the generalization. A good rule of thumb requires the size of the training sample to be of the same order of magnitude of the ratio between:

(a) the total number of free parameters (i.e., synaptic weights and biases) in the network,

(b) the fraction of classification errors permitted on test data (e.g., the mean square value of the estimation error).

\subsection{Cross validation}

A computationally efficient training and the evaluation of good generalization can be performed with the statistical tool of cross-validation. The available data are randomly divided in the following subsets:

1. training samples subset, 
2. generalization (or validation, or early stopping) samples subset,

3. test samples subset.

For instance, every ten epochs the training is halted and the validation error (or the cost function) is evaluated with the partially trained network over the validation set. Then the training is resumed.

This continues until the learning curve of the error for the validation set reaches a local minimum, a

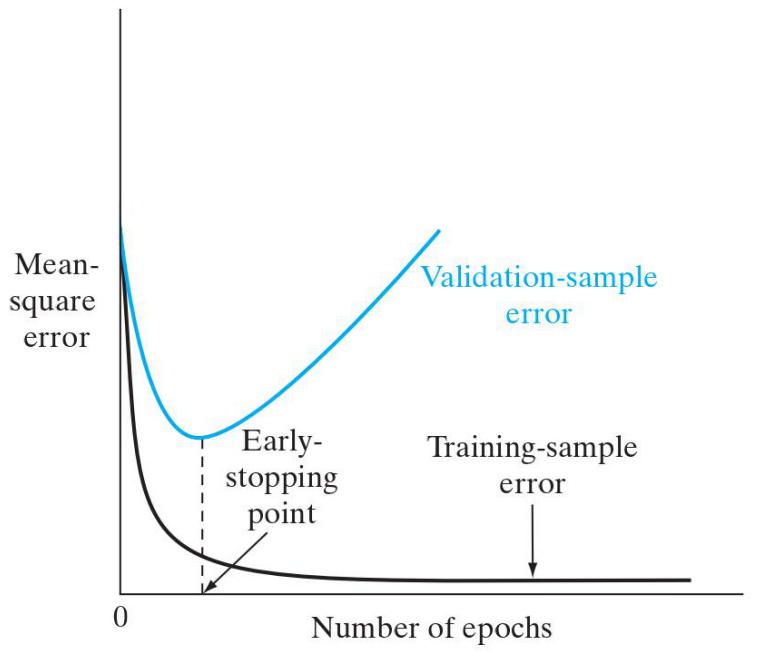

Figure 5.8: Early stopping [67]. The advantage in computational time is evident from the image.

condition that should happen sooner (i.e., in a minor number of loops, see Fig. 5.8) than the falling of the learning curve for the training set under the prescribed limit. At this point the output of the trained network is evaluated over the test subset to evaluate its generalization performances.

Also the standard regression models (i.e., Bayesian and Gaussian) are similarly evaluated.

\subsection{ANNs training for DEM identification}

In their work, Vaferi et al. [135] suggest an effective algorithm for MLPNN regression. We followed their instructions, see Chapter 8

Further, the quality of the ANN data had to be examined critically. Haykin [67] suggested considering the quality of:

(a) ANN training process,

(b) the subsequent data generation based on the inputs provided.

Task (a) is particularly important when dealing with experimental training data, and usually addressed by noise-corrupted pattern calibration and by comparison with standard statistical methods. However, our training pool was numerical and extensive, and the particles in our simulations were inserted using a random seed value. For vast amounts of training data, the effect of noise-corrupted patterns is negligible, see Benyahia and Galvin [19], Haykin 67].

Thus, in our work task (b) was more challenging. Once trained, the ANN were fed combinations of DEM parameters. We tried different methods to generate these combinations. Our first attempt consisted of assigning parameters to the investigated variables in even increments from the minimum to the maximum values. For example, the $C O R$ ranged from 0.5 to 0.9 , and thus the first value was 0.5 , the second 0.508163 , and so on.

To increase generalization, we decided to follow a different approach: random value generators created the 
number of required values in the defined ranges for each parameter investigated. These were combined and used as input.

\subsection{Comparison of regression methods}

A comparison of the regression methods, based on their cost functions, is provided in 5.1 . In Section 8.3

\begin{tabular}{|c|c|c|c|}
\hline & Bayesian & Gaussian & $\mathrm{ANN}$ \\
\hline \multirow[t]{2}{*}{ Cost function } & maximum & maximum & scaled \\
\hline & $\begin{array}{l}\text { likelihood } \\
\text { estimation }\end{array}$ & $\begin{array}{l}\text { likelihood } \\
\text { estimation }\end{array}$ & $\begin{array}{l}\text { conjugated } \\
\text { gradient }\end{array}$ \\
\hline
\end{tabular}

Table 5.1: Regression methods comparison.

we compared the methods considering their average classification errors, given the test samples subsets. 


\section{Part III}

\section{Identification of DEM parameters}




\section{Chapter 6}

\section{Experimental Characterization}

In the industrial processes we presented in Chapter 2 and in Part IV the following materials:

- coke,

- iron ore,

- limestone,

- sinter,

are involved, which can be furtherly divided according to the diameter of the particles in two categories:

- fine, until 3.15 millimiters;

- coarse, larger than 3.15 millimiters.

We followed a series of standardized procedures to characterize these particles, as explained by various authors (Carson and Wilms [33, Das et al. [43], Ganesan et al. [47, Lumay et al. [95], Moysey et al. [103], Simsek et al. [126, Snider [128]). These experimental data were later used to determine DEM] numerical parameters, as explained in Chapters 7 and 8 .

For our investigations we focused on particle-particle $(p-p)$ interactions, and the bulk behaviour representative values summarized in Table 6.1. According to Kloss et al. [83], they are the most relevant for the large, dense conditions we researched.

\begin{tabular}{lcc}
\hline & acronym & formula \\
\hline bulk density & $\rho_{b}$ & $\frac{\text { mass of the bulk }}{\text { volume of the bulk }}$ \\
steady-state flow/pre-shear coefficient of internal friction & $\mu_{p s h}$ & $\frac{\tau_{p s h}}{\sigma_{n, p s h}}$ \\
incipient flow/shear coefficient of internal friction & $\mu_{s h}$ & $\frac{\tau_{s h}}{\sigma_{n, s h}}$ \\
static angle of repose & AoR & slope's angle \\
\hline
\end{tabular}

Table 6.1: Values representative of bulk behaviour.

\subsection{Particle Distribution}

Determine the particles distribution is the first task in bulk materials characterization, see Chen et al. [34], Johanson [77], Schwedes [123], Yoshida et al. [145]. Thus, we sieved each material investigated with an electrically tilting siever. We proceded in a sequence from the largest to the smallest sieving diameter $(6.3,5.6,3.55,2,1.6,1.25,1,0.8,0.63,0.5,0.315,0.25,0.0001$ millimiters $)$. The results can be seen in Table 6.2. 


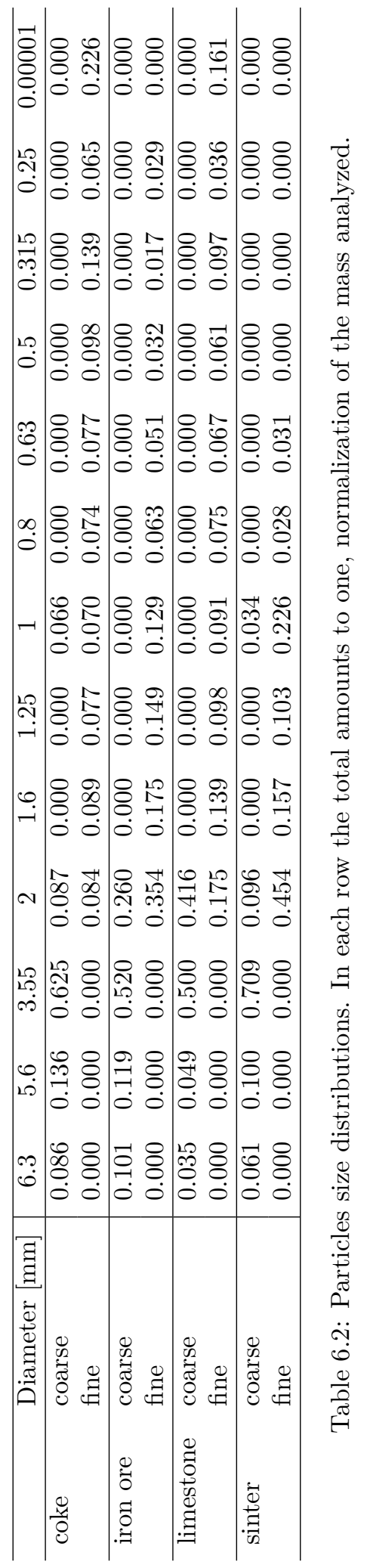




\subsection{Drained angle of repose (p-p) - small scale}

Later, we performed $p-p$ drained angle of repose (AoR tests Grasselli and Herrmann [52, Grasselli et al. [53, Zhou et al. [146]. Drained, because each sample was completely dry and moistless. Schulze [120] defines angle of repose the angle of the slope of a bulk material sample when it reaches steady-state after deposition.

In our test, a sample was deposited on a 20 centimeters diameter plate (hence, small scale) with liftable boundary called static AoR tester, see Fig. 6.1. Once the particles were in position, the boundary was lifted, allowing some particles to drop. Once stabilized, the AoR was measured eight times using a digital protractor at different positions of the heap. The average of the measurements gave the fourth bulk value (see Table 6.1). Note that, since the experiments were performed only for relatively large size bulk solids, the compaction condition in the initial state was not critical to the final result of this test.

On the left column of Table 6.3 the results of the tests performed on the various materials can be seen.

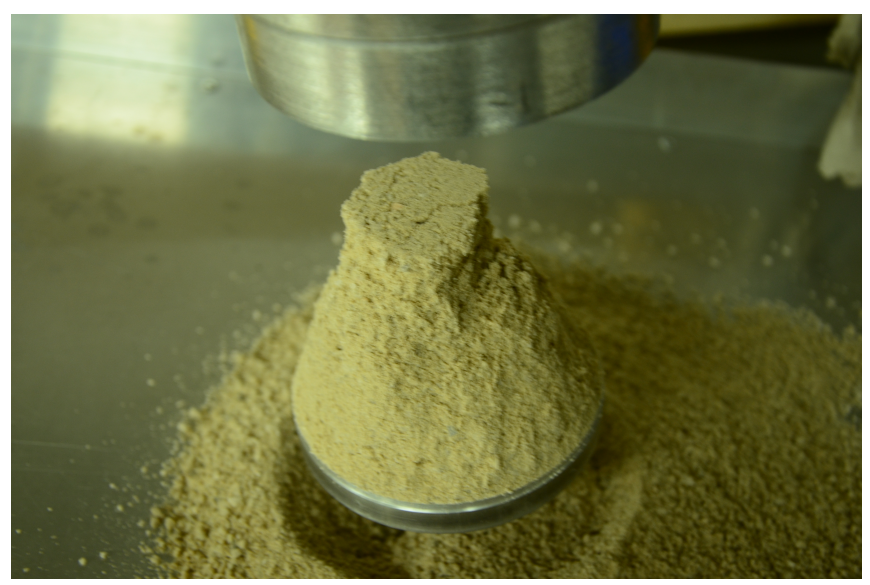

(a) Laboratory setup of the drained angle of repose.

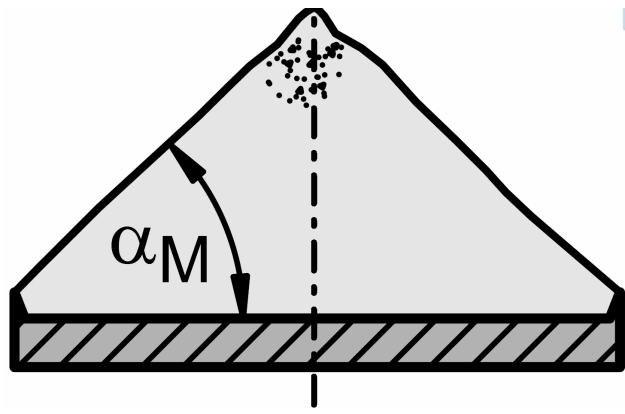

(b) Drained angle of repose tester [120].

Figure 6.1: AoR experimental tester.

\begin{tabular}{ll|ll}
\hline & & \multicolumn{2}{|c}{ AOR [ $\left.{ }^{\circ}\right]$} \\
& scale & small & large \\
\hline coke & coarse & 40.90 & \\
& fine & 42.60 & \\
\hline iron ore & coarse & 44.15 & 34.96 \\
ore & fine & 43.00 & \\
\hline limestone & coarse & 37.75 & \\
& fine & 44.50 & \\
\hline sinter & coarse & 39.05 & 35.81 \\
& fine & 38.85 & \\
\hline
\end{tabular}

Table 6.3: AOR experimental values.

\subsection{Drained angle of repose (p-p) - large scale}

At the Leoben VAS facility a new rotating double chute was used: two large scale dynamic angle of repose tests were performed. In this case coarse iron ore and sinter were poured from a chute, like in Fig. 6.2a Once removed the safety cylindrical container, useless for this test (Fig. 6.2b), a laserscan apparatus 
proceeded to determine the large scale AoR The results can be gound in the right column of Table 6.3 We can see how the values are inferior to their static counterparts. The two main reasons for this behaviour are, of course, the dinamicity of the test and the large scale of the setup.

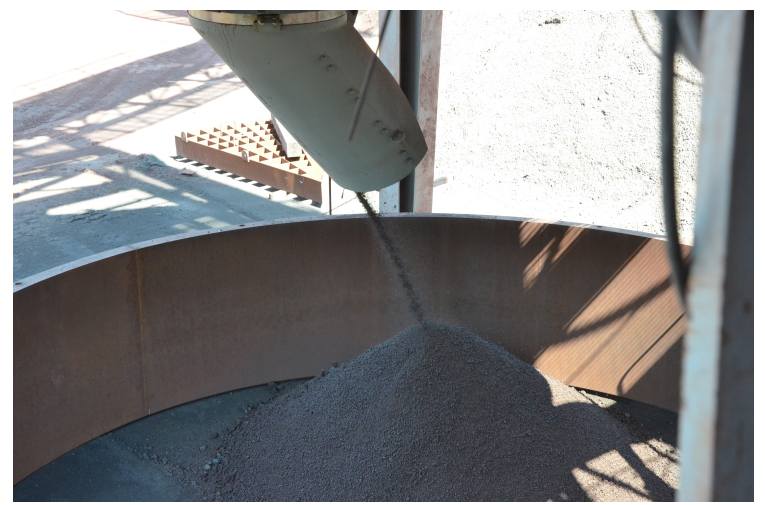

(a) Material pouring from an above chute.

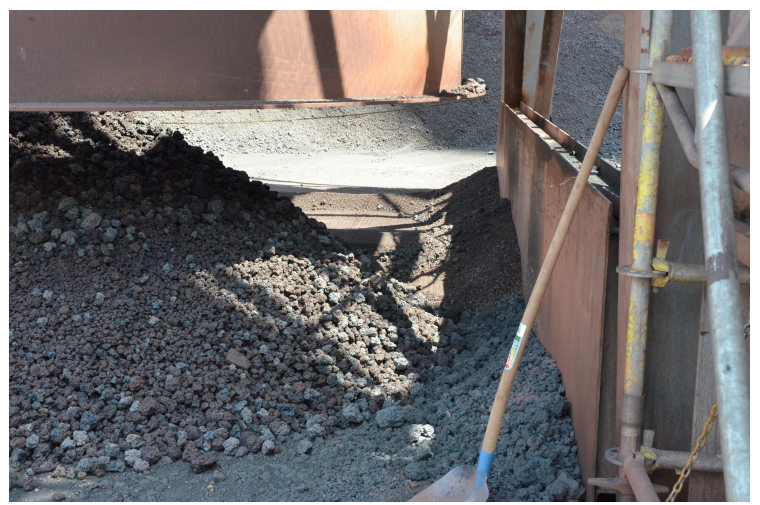

(b) Scale of the resulting pile.

Figure 6.2: AoR large scale test. Images of the tests performed at the Voestalpine Leoben plant.

\subsection{Jenike Shear Cell tester}

The third step of the procedure was using shear testers - a simplified Jenike shear cell tester (JSCT, Fig. 6.3c see ASTM [7]) and a Schulze ring shear cell tester (SCT] see ASTM [8]) to characterize particle flow properties, especially the complete yield locus. Compared to the original [JSCT in Fig. 6.3a, we defined our tester in Fig. 6.3b simplified because it is a prototype designed for fast characterization (see Aigner et al. [2]). Each experiment was performed on a fresh material sample.

In the tests with the simplified JSCT a representative sample of bulk solid was placed in a shear cell of 104 millimiters diameter. This specimen was pre-consolidated by twisting the shear cell cover while applying a compressive load (from 0.488 to $1.379 \mathrm{~kg}$ ) normal to it.

Since this was a simplified tester, the specimen was then sheared with the same normal load at a constant velocity. In fact, the shear to failure phase was missing in these simplified tests, and the consolidation phase was not completely serialized. The steady-state flow horizontal stress (Fig. 6.3d) is called pre-shear stress $\left(\tau_{p s h}\right)$. Knowing the normal stress, it gives (Eq. 6.1 the angle of internal friction of the pre-shear phase $\left(\phi_{e}-p s h\right)$, our first flowability value [119]:

$$
\begin{aligned}
\phi_{e-p s h} & =\arctan \left(\frac{\tau_{p s h}}{\sigma_{n, p s h}}\right), \\
\mu_{p s h} & =\tan \left(\phi_{e-p s h}\right) .
\end{aligned}
$$

The values obtained in the tests are summarized in Table 6.4.

\subsection{Schulze Ring Shear Cell tester (p-p)}

A representative sample of bulk solid was placed in a Schulze ring shear cell tester (SCT) of specified dimensions (external radius $=100 \mathrm{~mm}$, internal radius $=50 \mathrm{~mm})$, see Fig. 6.4a $([68,69$, 117, 118, 121, 122, 124]). 


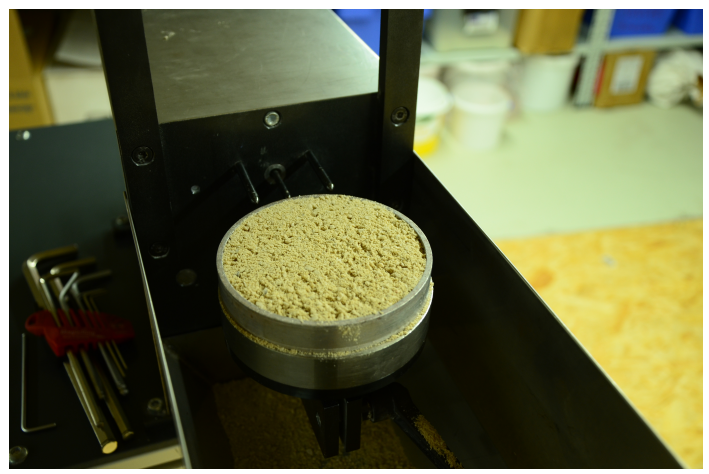

(a) Laboratory setup of the Jenike shear cell tester.

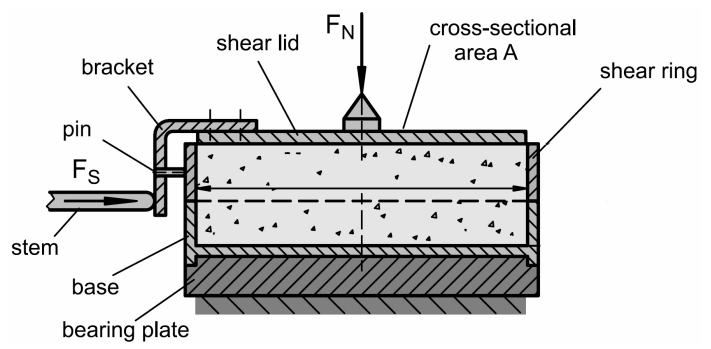

(c) Jenike shear cell tester layout 120.

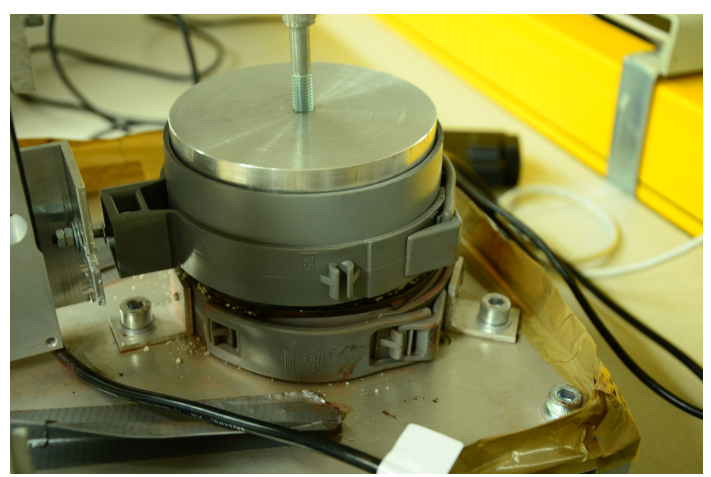

(b) Laboratory setup of the simplified Jenike shear cell tester.

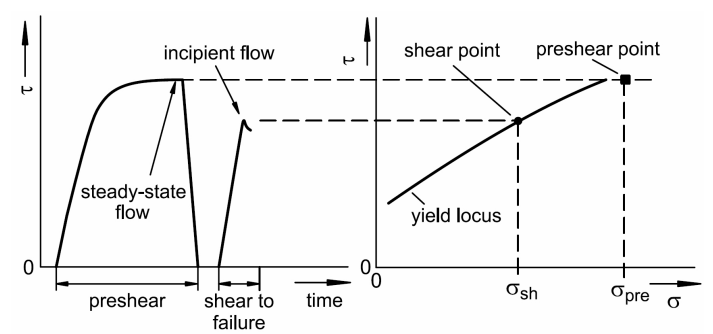

(d) Jenike shear cell tester diagram of the shear stress, on the $\mathrm{y}$ axis, over time, on the $\mathrm{x}$ axis 120 .

Figure 6.3: JSCT.

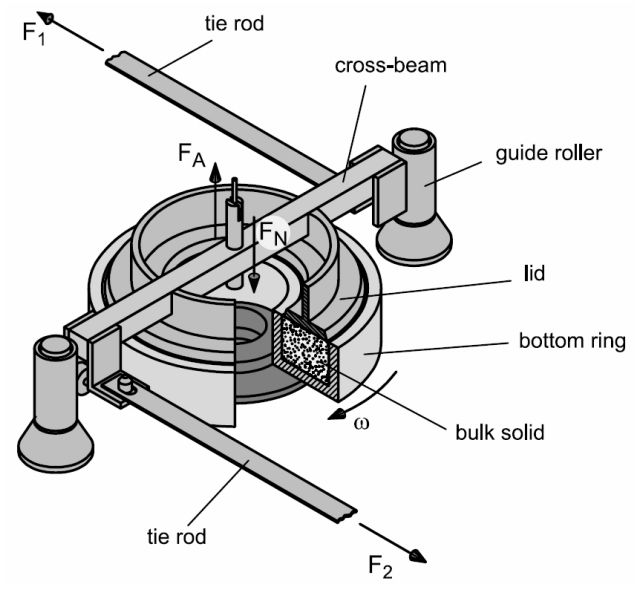

(a) Ring shear cell tester layout.

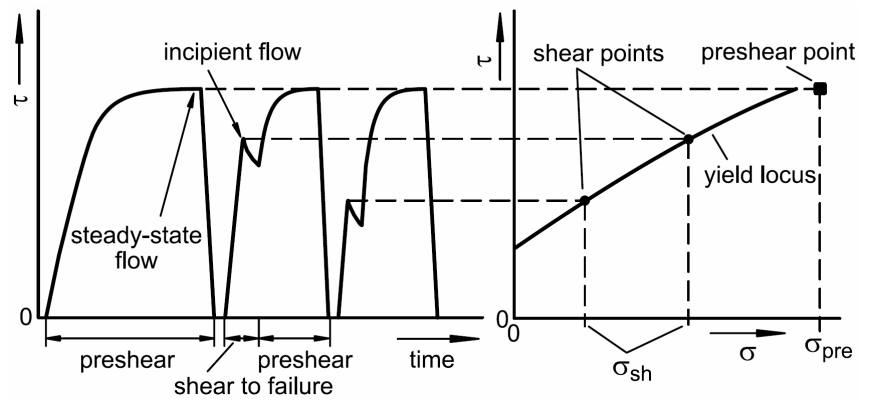

(b) Ring shear cell diagram of the shear stress, on the y axis, over time, on the $\mathrm{x}$ axis.

Figure 6.4: SCT experimental tester [120]. 


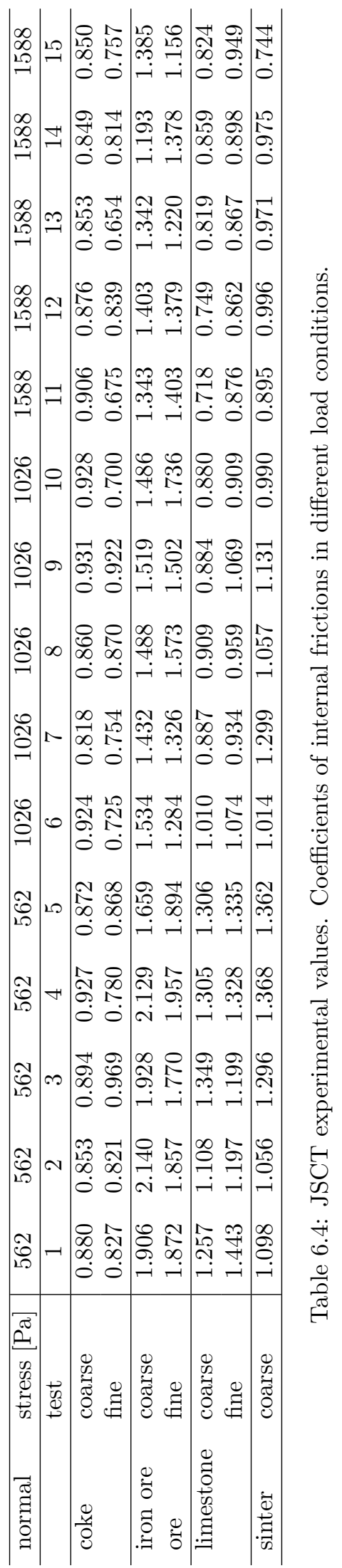




\subsubsection{SRSCT procedure}

A normal load was applied to the cover. As soon as the lid touched the sample, its position was calculated. Together with the area of the ring, the total volume can be calculated, and subsequently the bulk density $\rho_{b}$

$$
\rho_{b}=\frac{\text { mass }}{\text { volume }}
$$

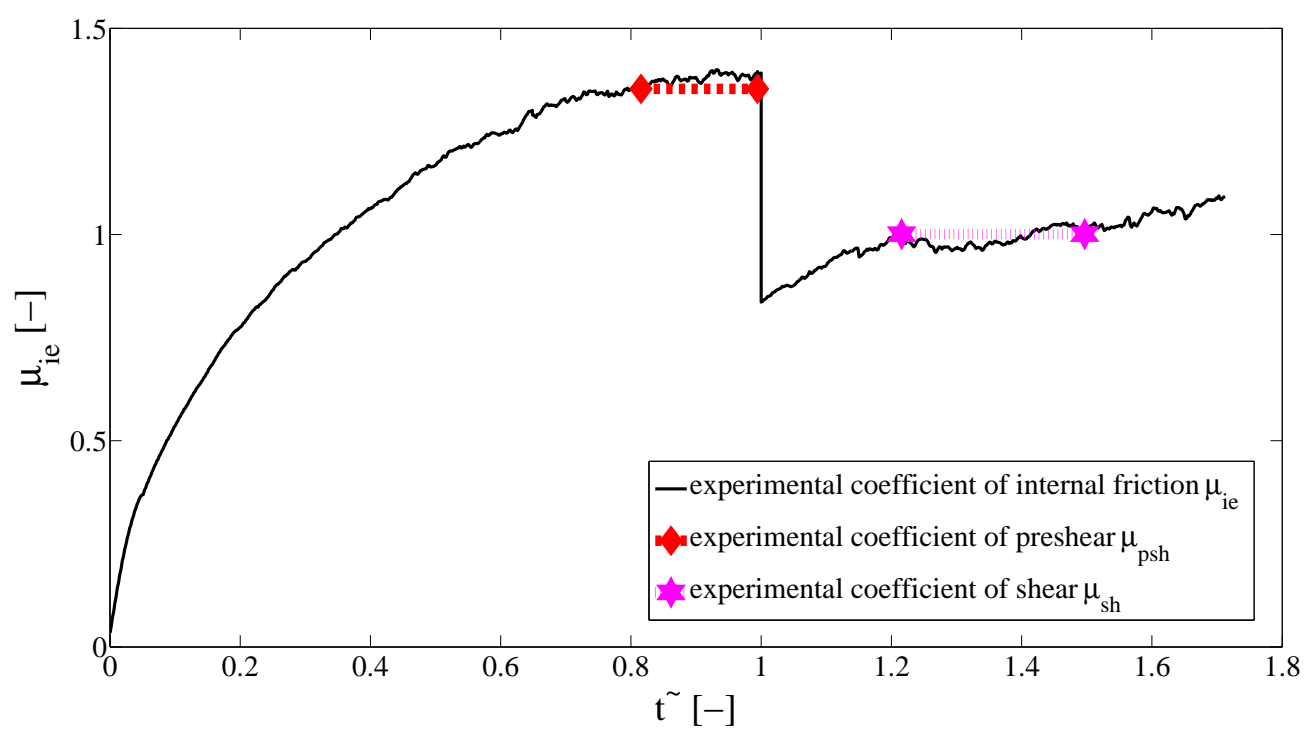

Figure 6.5: Experimental shear-cell tester stress path $-\sigma_{n}=10000 \mathrm{~Pa}$.

the first bulk value of the sample, was obtained. Later, we tried to obtained the bulk values indicated in Table 6.1. slightly different from those indicated in Fig. 6.4b

A representative stress path can be seen in Fig. 6.5. Time was normalized: $\tilde{t}=t / t_{\text {change }}$, where $t_{\text {change }}$ is the point in time at which the normal stress $\left(\overline{\sigma_{n}}\right)$ was modified during the tests. After the $\left.\rho_{b}\right]$ identification, the $\sigma_{n}$ was kept constant at e.g. $10,000 \mathrm{~Pa}$ until $\tilde{t}=1$. The specimen was in this way pre-sheared until a steady-state shear value was reached from $\tilde{t}=0.91$ to $\tilde{t}=1$. The steady-state flow horizontal stress is called steady-state flow/pre-shear stress. If the normal stress is known, it provides (Eq. 6.1) the angle of internal friction of the pre-shear phase $\left(\phi_{e-p s h}\right)$, and consequently the pre-shear coefficient of internal friction $\left(\mu_{p s h}\right)$, the second bulk value, see Eq. 6.1] [119].

At $\tilde{t}=1$ the normal stress and the angular velocity were then immediately reduced to zero. Subsequently, the specimen was sheared under a fraction $\left(\tau_{\%}\right)($ e.g. $80 \%)$ of the first normal load until the shear force reached a a second plateau. Both the pre-shear and shear phases were executed at constant velocity. We define the horizontal stress at the shear force peak as the average shear stress in this second plateau, thus obtaining the incipient flow/shear coefficient of internal friction $\mu_{s h}$, third bulk value (Eq. 6.3) [119]:

$$
\begin{aligned}
\phi_{e-s h} & =\arctan \left(\frac{\tau_{s h}}{\sigma_{n, s h}}\right), \\
\mu_{s h} & =\tan \left(\phi_{e-s h}\right) .
\end{aligned}
$$

Between two and five different pre-shear normal loads were applied in each experiment $(1,000,2,000$, $5,000,10,000$, and $15,000 \mathrm{~Pa})$. For each we used a normal load proportional to the initial one $\left(\overline{\tau_{\%}}\right)$, increasing from stage one $(40 \%)$ to stage four $(100 \%)$ with two escalating intermediate stages $(60 \%$ and $80 \%)$. Each experiment was performed on a fresh material sample. 


\subsubsection{SRSCT results}

The tipical stress path, normalized into coefficient of internal friction on the y-axis and normalized time on the x-axis, can be seen in Fig. 6.5

We then collected the results of the 26 tests performed according to the load conditions of Table 6.5 in Tables 6.6 and 6.7. Each of the four column represents one load stage. Further, $\rho_{b-100}$ has been used as bulk value $\rho_{b}$. Intuitevely, $\phi_{e-100}$ was the $\phi_{e-p s h}$, while $\phi_{e-40}, \phi_{e-60}$, and $\phi_{e-80}$ represented the $\phi_{e-s h}$ in the different stages. The experimental values collected were used for the identification of the microscopic numerical parameters (further details in Chapter 8).

\begin{tabular}{|c|c|c|c|c|c|c|c|c|c|c|}
\hline & & test & $\sigma_{A b 40}$ & $\sigma_{A b 60}$ & $\sigma_{A b 80}$ & $\sigma_{A b 100}$ & $\tau_{A b 40}$ & $\tau_{A b 60}$ & $\tau_{A b 80}$ & $\tau_{A b 100}$ \\
\hline \multirow[t]{2}{*}{ coke } & coarse & 1 & 819 & 1221 & 1621 & 2020 & 1246 & 1376 & 1551 & 1841 \\
\hline & & 2 & 2020 & 3021 & 4020 & 5020 & 2296 & 3243 & 3853 & 4486 \\
\hline \multirow[t]{3}{*}{ coke } & fine & 1 & 828 & 1230 & 1629 & 2029 & 1154 & 1418 & 1726 & 1819 \\
\hline & & 2 & 2030 & 3031 & 4029 & 5030 & 2347 & 3079 & 3778 & 4064 \\
\hline & & 3 & 4030 & 6032 & 8031 & 10031 & 4261 & 6028 & 7367 & 7939 \\
\hline \multirow[t]{3}{*}{ iron ore } & coarse & 1 & 884 & 1286 & 1686 & 2085 & 1954 & 1687 & 2092 & 2173 \\
\hline & & 2 & 4086 & 6088 & 8088 & 10087 & 4507 & 6083 & 6990 & 8375 \\
\hline & & 3 & 2087 & 3088 & 4087 & 5087 & 2524 & 5098 & 4273 & 4606 \\
\hline \multirow[t]{5}{*}{ iron ore } & fine & 1 & 488 & 689 & 888 & 1090 & 594 & 755 & 939 & 1027 \\
\hline & & 2 & 887 & 1288 & 1687 & 2087 & 1248 & 1449 & 1800 & 1948 \\
\hline & & 3 & 2092 & 3093 & 4092 & 5092 & 2285 & 3084 & 3956 & 4343 \\
\hline & & 4 & 4094 & 6096 & 8096 & 10095 & 4830 & 6442 & 7820 & 8805 \\
\hline & & 5 & 6093 & 9091 & 12091 & 15092 & 6705 & 9640 & 11797 & 12885 \\
\hline \multirow[t]{2}{*}{ limestone } & coarse & 1 & 849 & 1251 & 1650 & 2050 & 1525 & 1946 & 1679 & 1718 \\
\hline & & 2 & 2049 & 3050 & 4049 & 5050 & 2180 & 2952 & 3779 & 4193 \\
\hline \multirow[t]{4}{*}{ limestone } & fine & 1 & 462 & 661 & 861 & 1063 & 673 & 853 & 957 & 1036 \\
\hline & & 2 & 862 & 1264 & 1663 & 2063 & 1000 & 1326 & 1694 & 1841 \\
\hline & & 3 & 2063 & 3064 & 4062 & 5063 & 2504 & 3225 & 4049 & 4449 \\
\hline & & 4 & 4062 & 6064 & 8063 & 10063 & 4691 & 5977 & 7639 & 8609 \\
\hline \multirow[t]{3}{*}{ sinter } & coarse & 1 & 455 & 655 & 855 & 1056 & 591 & 722 & 734 & 751 \\
\hline & & 2 & 857 & 1258 & 1657 & 2057 & 1153 & 1448 & 1554 & 1679 \\
\hline & & 3 & 2058 & 3060 & 4058 & 5059 & 2479 & 2853 & 3419 & 4244 \\
\hline \multirow[t]{4}{*}{ sinter } & fine & 1 & 467 & 667 & 866 & 1068 & 703 & 823 & 989 & 1059 \\
\hline & & 2 & 868 & 1270 & 1669 & 2069 & 928 & 1371 & 1673 & 1818 \\
\hline & & 3 & 2070 & 3070 & 4069 & 5070 & 2696 & 4322 & 3758 & 4352 \\
\hline & & 4 & 4070 & 6071 & 8071 & 10070 & 4145 & 6729 & 9423 & 8232 \\
\hline
\end{tabular}

Table 6.5: [SCT experimental values 1. Normal and tangential stresses in different load conditions. 


\begin{tabular}{|c|c|c|c|c|c|c|c|c|c|c|}
\hline & & test & $\Delta h_{40}$ & $\Delta h_{60}$ & $\Delta h_{80}$ & $\Delta h_{100}$ & $\rho_{b-40}$ & $\rho_{b-60}$ & $\rho_{b-80}$ & $\rho_{b-100}$ \\
\hline \multirow{2}{*}{ coke } & \multirow[t]{2}{*}{ coarse } & 1 & -0.542 & -0.302 & -0.09 & 0 & 516 & 519 & 522 & 519 \\
\hline & & 2 & -1.329 & -0.941 & -0.838 & 0 & 518 & 523 & 524 & 521 \\
\hline \multirow[t]{3}{*}{ coke } & \multirow[t]{3}{*}{ fine } & 1 & 4.136 & 4.166 & 4.089 & 0 & 738 & 739 & 737 & 738 \\
\hline & & 2 & 5.465 & 5.361 & 5.253 & 0 & 771 & 769 & 766 & 769 \\
\hline & & 3 & 5.284 & 5.214 & 5.067 & 0 & 787 & 786 & 782 & 785 \\
\hline \multirow[t]{3}{*}{ iron ore } & \multirow[t]{3}{*}{ coarse } & 1 & -2.207 & -1.979 & -1.767 & 0 & 2176 & 2188 & 2200 & 2188 \\
\hline & & 2 & -1.548 & -1.629 & -1.433 & 0 & 2225 & 2221 & 2231 & 2226 \\
\hline & & 3 & -1.491 & -1.526 & -1.318 & 0 & 2227 & 2225 & 2236 & 2229 \\
\hline \multirow[t]{5}{*}{ iron ore } & \multirow[t]{5}{*}{ fine } & 1 & -0.893 & -0.74 & -0.784 & 0 & 2267 & 2276 & 2273 & 2272 \\
\hline & & 2 & -0.696 & -0.742 & -0.86 & 0 & 2235 & 2232 & 2225 & 2231 \\
\hline & & 3 & -0.042 & -0.059 & -0.051 & 0 & 2359 & 2358 & 2358 & 2358 \\
\hline & & 4 & 0.318 & 0.317 & 0.344 & 0 & 2426 & 2426 & 2427 & 2426 \\
\hline & & 5 & 0.829 & 0.753 & 0.805 & 0 & 2349 & 2344 & 2347 & 2347 \\
\hline \multirow[t]{2}{*}{ limestone } & \multirow[t]{2}{*}{ coarse } & 1 & -2.693 & -2.746 & -2.669 & 0 & 1276 & 1275 & 1277 & 1276 \\
\hline & & 2 & -2.183 & -2.032 & -1.95 & 0 & 1267 & 1272 & 1274 & 1271 \\
\hline \multirow[t]{4}{*}{ limestone } & \multirow[t]{4}{*}{ fine } & 1 & 0.059 & 0.045 & -0.069 & 0 & 1586 & 1586 & 1581 & 1584 \\
\hline & & 2 & 0.599 & 0.518 & 0.442 & 0 & 1608 & 1605 & 1602 & 1605 \\
\hline & & 3 & 1.424 & 1.412 & 1.308 & 0 & 1617 & 1617 & 1612 & 1615 \\
\hline & & 4 & 1.359 & 1.318 & 1.162 & 0 & 1603 & 1602 & 1595 & 1600 \\
\hline \multirow[t]{3}{*}{ sinter } & \multirow[t]{3}{*}{ coarse } & 1 & -2.637 & -2.661 & -2.708 & 0 & 1419 & 1418 & 1417 & 1418 \\
\hline & & 2 & -2.081 & -2.145 & -2.249 & 0 & 1466 & 1464 & 1460 & 1463 \\
\hline & & 3 & -3.268 & -3.076 & -3.236 & 0 & 1501 & 1508 & 1503 & 1504 \\
\hline \multirow[t]{4}{*}{ sinter } & \multirow[t]{4}{*}{ fine } & 1 & -2.002 & -1.909 & -1.958 & 0 & 1716 & 1720 & 1718 & 1718 \\
\hline & & 2 & -1.001 & -1.014 & -1.082 & 0 & 1760 & 1760 & 1757 & 1759 \\
\hline & & 3 & -0.568 & -0.617 & -0.656 & 0 & 1785 & 1783 & 1781 & 1783 \\
\hline & & 4 & -0.025 & -0.038 & -0.115 & 0 & 1803 & 1803 & 1799 & 1802 \\
\hline
\end{tabular}

Table 6.6: SCT experimental values 2. Vertical displacements and the bulk densities in the load conditions of Table 6.5 


\begin{tabular}{|c|c|c|c|c|c|c|}
\hline & & test & $\phi_{e-100}$ & $\phi_{e-40}$ & $\phi_{e-60}$ & $\phi_{e-80}$ \\
\hline \multirow[t]{2}{*}{ coke } & \multirow[t]{2}{*}{ coarse } & 1 & 0.9114 & 1.4756 & 1.0724 & 1.0322 \\
\hline & & 2 & 0.8936 & 1.1426 & 1.0568 & 0.9686 \\
\hline \multirow[t]{3}{*}{ coke } & \multirow[t]{3}{*}{ fine } & 1 & 0.8965 & 1.3119 & 1.1963 & 1.0810 \\
\hline & & 2 & 0.8080 & 1.1592 & 1.0200 & 0.9315 \\
\hline & & 3 & 0.7914 & 1.0743 & 1.0127 & 0.8903 \\
\hline \multirow[t]{3}{*}{ iron ore } & \multirow[t]{3}{*}{ coarse } & 1 & 1.0422 & 2.0971 & 1.3672 & 1.2524 \\
\hline & & 2 & 0.8303 & 1.0771 & 0.9502 & 0.9270 \\
\hline & & 3 & 0.9054 & 1.2179 & 1.6178 & 1.0593 \\
\hline \multirow[t]{5}{*}{ iron ore } & \multirow[t]{5}{*}{ fine } & 1 & 0.9422 & 1.1702 & 1.1140 & 1.0798 \\
\hline & & 2 & 0.9334 & 1.4433 & 1.1321 & 1.0328 \\
\hline & & 3 & 0.8529 & 1.0779 & 1.0013 & 0.9756 \\
\hline & & 4 & 0.8722 & 1.1340 & 1.0805 & 0.9816 \\
\hline & & 5 & 0.8538 & 1.1094 & 1.0640 & 0.9643 \\
\hline \multirow[t]{2}{*}{ limestone } & \multirow[t]{2}{*}{ coarse } & 1 & 0.8380 & 1.8020 & 1.3885 & 1.1236 \\
\hline & & 2 & 0.8303 & 1.0500 & 0.9866 & 0.9275 \\
\hline \multirow[t]{4}{*}{ limestone } & \multirow[t]{4}{*}{ fine } & 1 & 0.9746 & 1.4717 & 1.3099 & 1.0828 \\
\hline & & 2 & 0.8924 & 1.2048 & 1.0380 & 0.9902 \\
\hline & & 3 & 0.8787 & 1.2630 & 1.0419 & 0.9664 \\
\hline & & 4 & 0.8555 & 1.1796 & 0.9890 & 0.9240 \\
\hline \multirow[t]{3}{*}{ sinter } & \multirow[t]{3}{*}{ coarse } & 1 & 0.7112 & 1.2959 & 1.0868 & 0.8729 \\
\hline & & 2 & 0.8162 & 1.3667 & 1.1486 & 0.9249 \\
\hline & & 3 & 0.8389 & 1.1331 & 0.8827 & 0.9373 \\
\hline \multirow[t]{4}{*}{ sinter } & \multirow[t]{4}{*}{ fine } & 1 & 0.9916 & 1.4048 & 1.2185 & 1.2329 \\
\hline & & 2 & 0.8787 & 1.0434 & 1.1084 & 0.9994 \\
\hline & & 3 & 0.8584 & 1.2805 & 1.4067 & 0.9399 \\
\hline & & 4 & 0.8175 & 1.0565 & 1.0635 & 1.1712 \\
\hline
\end{tabular}

Table 6.7: SCT experimental values 3. Coefficients of internal frictions in the load conditions of Table 6.5 


\section{Chapter 7}

\section{Numerical Simulation}

Cundall and Strack [42] define a DEM] numerical simulation as a computational approximation of a real particulate material, represented as an ensemble of discrete, or distinct, elements, which interact among each other according to defined contact laws. Thus, under given hypotheses, we could use this simulation method to investigate the nature and the behaviour of any granular medium.

\subsection{Software}

We used the public open source DEM]software[LIGGGHTS as simulation toolbox. Berger et al. [20, Kloss et al. [83] demonstrated its effectiveness. Especially, it is an highly parallelized software, which allowed us to perform our simulations on large scale clusters.

Performing a simulation with LIGGGHTS consists of two steps:

1. write a script, stating the involved domain, the chosen contact laws, and the microscopic parameters of these laws;

2. running the script in bash with single or multiple cores.

\subsection{Microscopic parameters}

In our work, we decided to utilize a single contact law for all the simulations performed (see Benvenuti et al. [17]).

Following the indications summarized in Section 4.4 we chose time steps between $1.29 \%$ and $1.53 \%$ of the Rayleigh time, depending on the particle density $\left(\overrightarrow{\rho_{p}}\right)$ and on the particle size. Furthermore, we kept constant the size distribution, which was obtained by experimental sieving, see Table 6.2

The DEM values for the Young's modulus (E) and the Poisson's coefficient ( $(\boldsymbol{D})$ were taken from the literature and kept identical for each simulation, see [133] and [85]. However, we reduced the former to increase the time step $(\underline{\Delta t})$, following the recommendations of Ai et al. 1]. The values can be found in Table 7.1

\begin{tabular}{ccc}
\hline $\begin{array}{c}\text { Young's } \\
\text { modulus } \\
{[M P a]}\end{array}$ & $\begin{array}{c}\text { Poisson's } \\
\text { ratio } \\
{[-]}\end{array}$ & $\begin{array}{c}\Delta t \\
{[\mathrm{~s}]}\end{array}$ \\
\hline 10 & 0.40 & $10^{-6}$ \\
\hline
\end{tabular}

Table 7.1: DEM fixed input values 


\subsection{Macroscopic parameters}

A Matlab script allowed us to extract from the simulation output the numerical values representative of bulk behaviour (hereafter called bulk values), see Table 6.1. for each DEM simulation parameter combination.

\subsection{Drained Angle of Repose Simulation}

In AoR simulations we sought to replicate meticulously the experimental setup explained in Section 6.2 considering both the plate and the liftable boundary, with the same domain size consideration as before see Fig. 7.1 The particles had the same properties as in the shear-cell simulation. The first phase was identical to that of the shear-cell simulation. After lifting the boundary, the particles formed a heap. An image post-processing software was used to obtain the average slope. In the AoR tests the average of the repose angles provided us with the fourth bulk value of Table 6.1

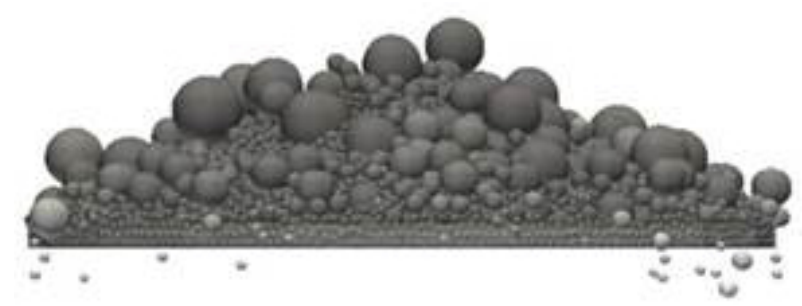

Figure 7.1: Angle of repose simulation. The experimental device was meticolously reproduced.

\subsection{Shear Cell Simulation}

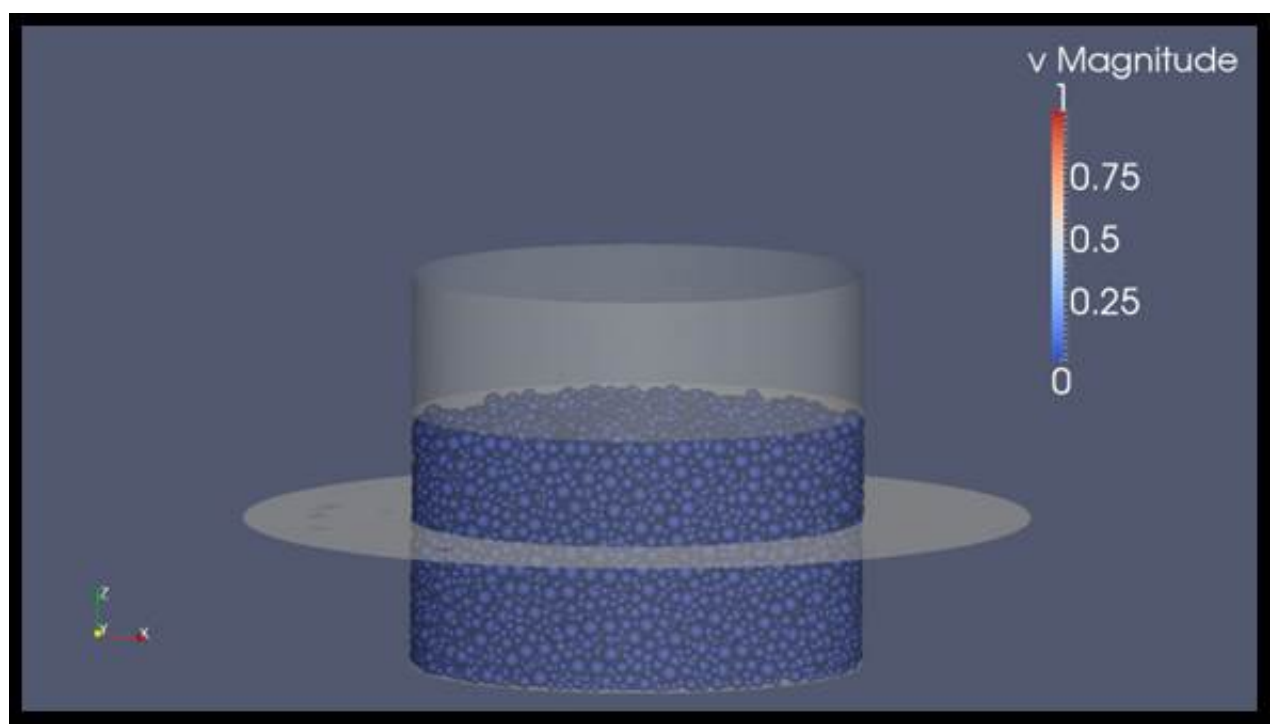

Figure 7.2: Shear cell simulation. The experimental device was meticolously reproduced.

LIGGGHTS the simulation toolbox we used, meets all the requirements of modelling the shear tester described in Section 6.4 First, it is capable of importing triangulated meshes of the two rings and a top 
lid. Since the real setup had a wall thickness, contact forces acting on a mesh are summed and can be saved, and thus shear force calculation is available out of the box. Second, the code can move a mesh with constant velocity as required for the measurement. To determine the shear stresses, the bulk solid had to be stressed with user-defined normal stresses. Therefore, a stress-controlled wall (servo-wall in LIGGGHTS) was applied to the lid.

Although the geometry differs, the $[\mathrm{SCT}$ was designed to obtain the same values for the shear stresses as the Jenike shear cell tester (JSCT), but with improved automation and reliability, see Schulze [119]. For this reason, the simulation setup was based on the JSCT see Fig. 7.2 As suggested by Aigner et al. [3] and Benvenuti et al. [18, the diameter and the height of the rings operated in the simulations had to be sufficiently large to avoid relevant wall effects. However, a larger domain increases the number of particles and thus simulation time. For this reason, we considered the cylinder dimension d dCylDp, which is proportional to the mean particle diameter, as an additional DEM parameter to be investigated.

\subsubsection{SCT simulation procedure}

A simulation run comprised four phases. First, the shear cell was filled with the granulate material, and it was allowed to settle. Then, the top lid was lowered and the first normal stress applied to the bulk solid. As in the experiment, the servo-wall allows calculating the position of the lid while the first particle is being touched. The distance between the lid and the bottom of the domain multiplied by the simulation area gave the total volume. Since the software already provided the total mass, we were able to calculate the bulk density $\left(\overline{\rho \rho_{b}}\right)$.

Next, the ring moved by a distance $l=0.1875 \cdot$ radius of the ring, and the required pre-shear force was measured. Finally, the normal load was reduced to a fraction of the initial load, the ring was moved again by a distance $l$, and the shear force was recorded. Unlike in the original experiment, the bottom ring was moved to facilitate the numerical simulation. The velocity of the ring displacement, and consequently the total simulation time, was determined by a trade-off between minimizing the normal load oscillation and optimizing computation time. The former requires (relatively) low velocity, and the latter requires high speed. We chose a constant velocity of $3 *$ (mean particle radius)/seconds, as the best trade-off. The normal stresses (pre-shear and shear phases) applied in each simulation were the same as in the experiments. The corresponding $\tau_{p s h}$ and $\tau_{s h}$ were calculated - as in the experiments - from the mean of the plateau.

\subsubsection{SCT Simulation Input Parameters}

The input DEM coefficients, $C o R, \mu_{s}, \mu_{r}, \rho_{p}$ and $\mathrm{dCylDp}$ (the cylinder dimension, proportional to the mean particle diameter), as indicated in Table 7.2 were constant in each simulation, but their combination differed between simulations. Further, dCylDp was used to evaluate the wall effect, but only $10 \%$ of the simulations had a dCylDp larger than 20. The normal stress $\sigma_{n}$ and its percentage during the incipient flow condition $\overline{\tau_{\%}}$ varied to replicate twelve shear-cell load conditions.

\subsubsection{SCT Simulation Output Parameters}

Each SCT simulation gave three of the bulk values indicated in Table 6.1 The first bulk value $(\sqrt[\rho b]{)})$ was provided directly. For correctly performed simulations, we observed a stress path as the example in Fig. 7.3 Time was normalized: $\tilde{t}=t / t_{\text {change }}$, where $t_{\text {change }}$ is the point in time at which the normal stress $\left.\sqrt{\sigma \sigma_{n}}\right)$ was modified during the tests. Until $\tilde{t}=1$, the $\sigma_{n}$ was kept constant at $10,000 \mathrm{~Pa}$. In Fig. 6.5. a plateau was reached at $\tilde{t}=0.91$. The coefficient of pre-shear $\left(\mu_{p s h}\right)$ was calculated as the average of the coefficient of internal friction $\left(\mu_{i e}\right)$ in this first plateau. At $\tilde{t}=1$, the $\sigma_{n}$ was reduced to $80 \%$ of its initial value, and soon after a second plateau developed. We obtained the coefficient of shear $\left(\overline{\mu_{s h}}\right)$ as the average of $\mu_{i e}$ in this second plateau. The stress paths between Fig. 7.2 and Fig. 6.5 agree well, especially 


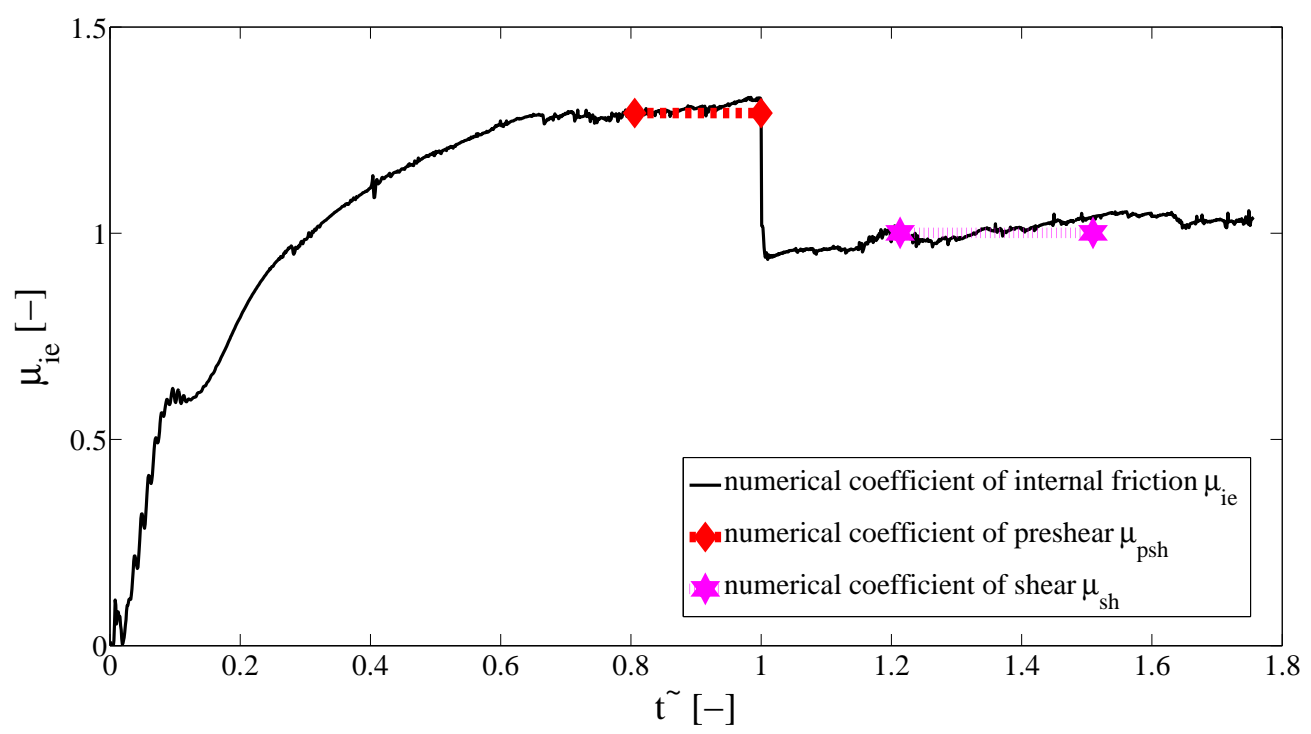

Figure 7.3: Numerical shear-cell tester stress path $-\sigma_{n}=10000 \mathrm{~Pa}$.

the plateaux. They were clearly relevant because the values representative of the bulk behaviours were collected there.

Together with the value from the AoR simulation, we were able to define the numerical bulk behaviour. All the results in this chapter are pure DEM, so they shoould be materials agnostic, given the same particle distribution, see Section 8.5.4.

\subsection{Influence of variations of input parameters}

In total, 2,400 shear cell and 400 static AoR simulations were run with the parameter combinations described in Table 7.2 with different particle size distributions for the various materials analyzed. The computational time amounted to 1 hour with 32 AMD cores for a benchmark shear-cell simulation and to 9 hours for a benchmark AoR simulation, both with 50,000 particles. Simulations with larger dCylDp required more time (e.g., about 12 hours for the shear cell with 400,000 particles ).

To better understand the effect of the variation of the input parameters, nine SCT simulations have been selected, and their stress paths shown in Fig. 7.4

\begin{tabular}{ccccc}
\hline$\mu_{s}$ & $\mu_{r}$ & {$[C o R$} & {$\left[\rho_{p}\right.$} & dCylDp \\
{$[-]$} & {$[-]$} & {$[-]$} & {$[k g / m 3]$} & {$[-]$} \\
\hline $0.4 / 0.6 / 0.8$ & $0.4 / 0.6 / 0.8$ & $0.5 / 0.7 / 0.9$ & $2500 / 3000 / 3500$ & $20 / 36 / 38 / 40$ \\
\hline
\end{tabular}

Table 7.2: DEM variable input values for Neural Networks training. 


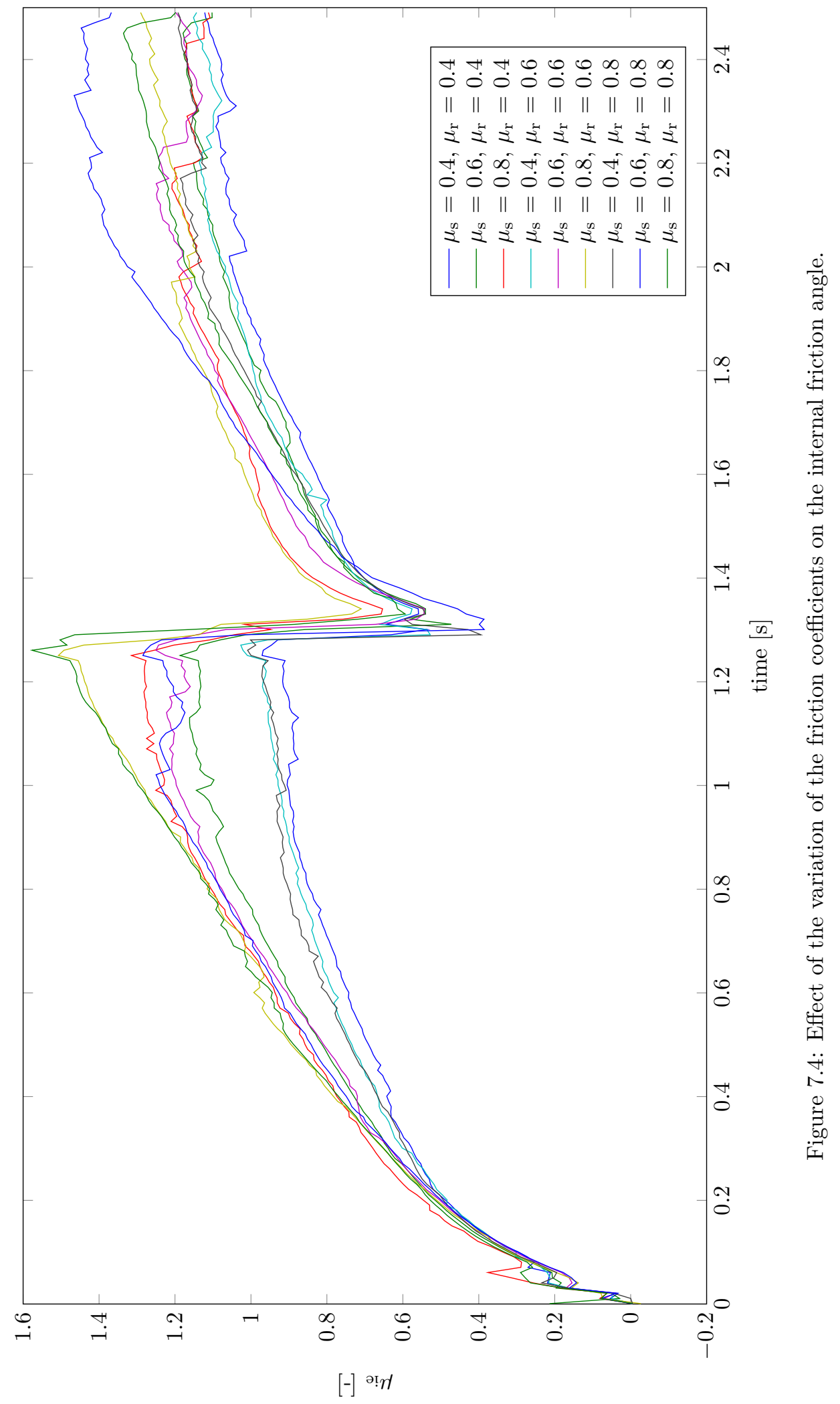




\section{Chapter 8}

\section{Artificial Neural Network Generalization}

\subsection{Principal Component Analysis (PCA) Results}

We evaluated the linear relationship between the microscopic and the macroscopic parameters in the training simulations with Matlab Principal Component Analysis. The results can be seen in Table 8.1 Sliding friction $\left(\overline{\mu_{s}}\right)$, rolling friction $\left(\overline{\mu_{r}}\right)$ and particle density $\left(\overline{\rho_{p}}\right)$ had the greatest influence on, respectively, the coefficient of pre-shear $\left(\mu_{p s h}\right)$, the angle of repose (AoR $)$ and the bulk density $\left(\underline{\rho_{b}}\right)$. Notably, $\rho_{p}$ was not used as a training parameter for $\mathrm{AoR}$ bulk behaviour.

In fact, we can see how each relationship is below the $100 \%$ necessary to claim a direct linear correlation. Thus, we demonstrated that we need more precise statistical tools to investigate the relationships and generalize the results.

\begin{tabular}{lcccccccc}
\hline & $\mu_{s}$ & $\mu_{r}$ & $\underline{C o R}$ & $\rho_{p}$ & $\mu_{s h}$ & $\mu_{p s h}$ & $\rho_{b}$ & AoR \\
\hline$\mu_{s}$ & 100.00 & 0.55 & 0.04 & 0.00 & 3.84 & 87.26 & 8.39 & 49.48 \\
$\mu_{r}$ & 0.55 & 100.00 & 0.15 & 0.00 & 58.92 & 33.70 & 3.10 & 60.20 \\
\hline \hline CoR & 0.04 & 0.15 & 100.00 & 0.00 & 15.52 & 0.57 & 1.71 & 21.35 \\
$\rho_{p}$ & 0.00 & 0.00 & 0.00 & 100.00 & 4.98 & 5.71 & 99.00 & 0.00 \\
\hline$\mu_{s h}$ & 3.84 & 58.92 & 15.52 & 4.98 & 100.00 & 26.03 & 9.52 & 0.00 \\
\hline$\mu_{p s h}$ & $\mathbf{8 7 . 2 6}$ & 33.70 & 0.57 & 5.71 & 26.03 & 100.00 & 4.33 & 0.00 \\
$\rho_{b}$ & 8.39 & 3.10 & 1.71 & $\mathbf{9 9 . 0 0}$ & 9.52 & 4.33 & 100.00 & 0.00 \\
AoR & 49.48 & $\mathbf{6 0 . 2 0}$ & 21.35 & 0.00 & 0.00 & 0.00 & 0.00 & 100.00 \\
\hline
\end{tabular}

Table 8.1: Values of linear relationship between variables considered multiplied by 100 .

\subsection{Regression statistic training concept}

Initially, as shown in Fig. 8.6 in the training phase (dashed lines) DEM simulations are performed with random initial input parameters. The behaviours obtained are used to train the following regression models:

- Bayesian linear,

- Gaussian non linear,

- Artificial Neural Network (ANN. 


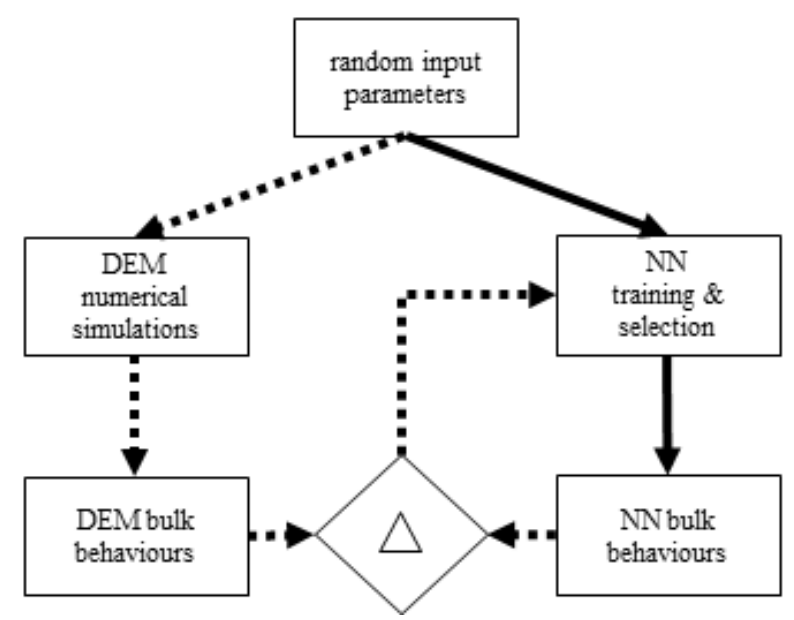

Figure 8.1: ANN training. Numerical simulations inputs and outputs are used to train the networks.

For all the three models training consists in a loop that continues until the difference between the outputs of each model and its samples is below the limit $(\Delta)$ (see Chapter 5 for more details).

Thus, we were able to use the DEM parameter combinations and their corresponding bulk values to train the models. Especially, we divided the samples in three pools: the first, with $70 \%$ of the samples, as training set, the second, with $15 \%$ of the samples, as generalization set, for early stopping, and the third, as test set, as suggested by Haykin (2009). The assignment of each sample to each pool was random.

\subsubsection{ANN training concept specifications}

The training of an ANN consists in defining the weights and the biases for each of its neuron. We first defined the typology of Artificial Neural Networks ANNs we used and the input we fed them, see Benvenuti et al. [17. Our ANNs have three different layers: the input layer has a number of neurons equal to the number of different inputs of the network, see Fig. 8.2 with the scheme of how the Multilayer Perceptron ANN $(M L P N N)$ derives one bulk-behaviour-dependent variable from the mutually independent simulation variables. The hidden (or central) layer's number of neurons was to be investigated. The output layer contains one neuron for the output. The transfer function for the neurons of the central layer is the tangential sigmoid, while the neuron of the output layer use the linear transfer function.

We started with all the DEM parameter combinations and their corresponding numerical $\mu_{\text {psh }}$ from the training set to create 36 ANNS that differed in their numbers of neurons in the hidden layer (between five to forty neurons). The generalization set was used to speed the training. We then determined the coefficient of determination $\underline{R}^{2}$,

$$
R^{2}=\frac{S S R}{S S T}=1-\frac{S S E}{S S T},
$$

between the bulk - macro behaviours in the output of the ANN and the test set simulations, which were not correlated with the remaining $70 \%$ used for the training. Thus, we could select for $\mu_{p s h}$ the ANN with the maximum $R^{2}$ again as suggested by Vaferi et al. [135], and we noted its number of neurons. We repeated the same $\mathrm{ANN}$ creation steps for $\mu_{s h}, \rho_{b}$ and AoR, obtaining one trained ANN for each bulk value.

\subsubsection{Sinter fine ANN training}

As said, we started with the sinter fine. The first bulk value ANN trained was the $\mu_{p s h}$, where we achieved a $R^{2}=0.96$ for an $\mathrm{ANN}$ with twenty neurons, a consistent agreement between the DEM and 

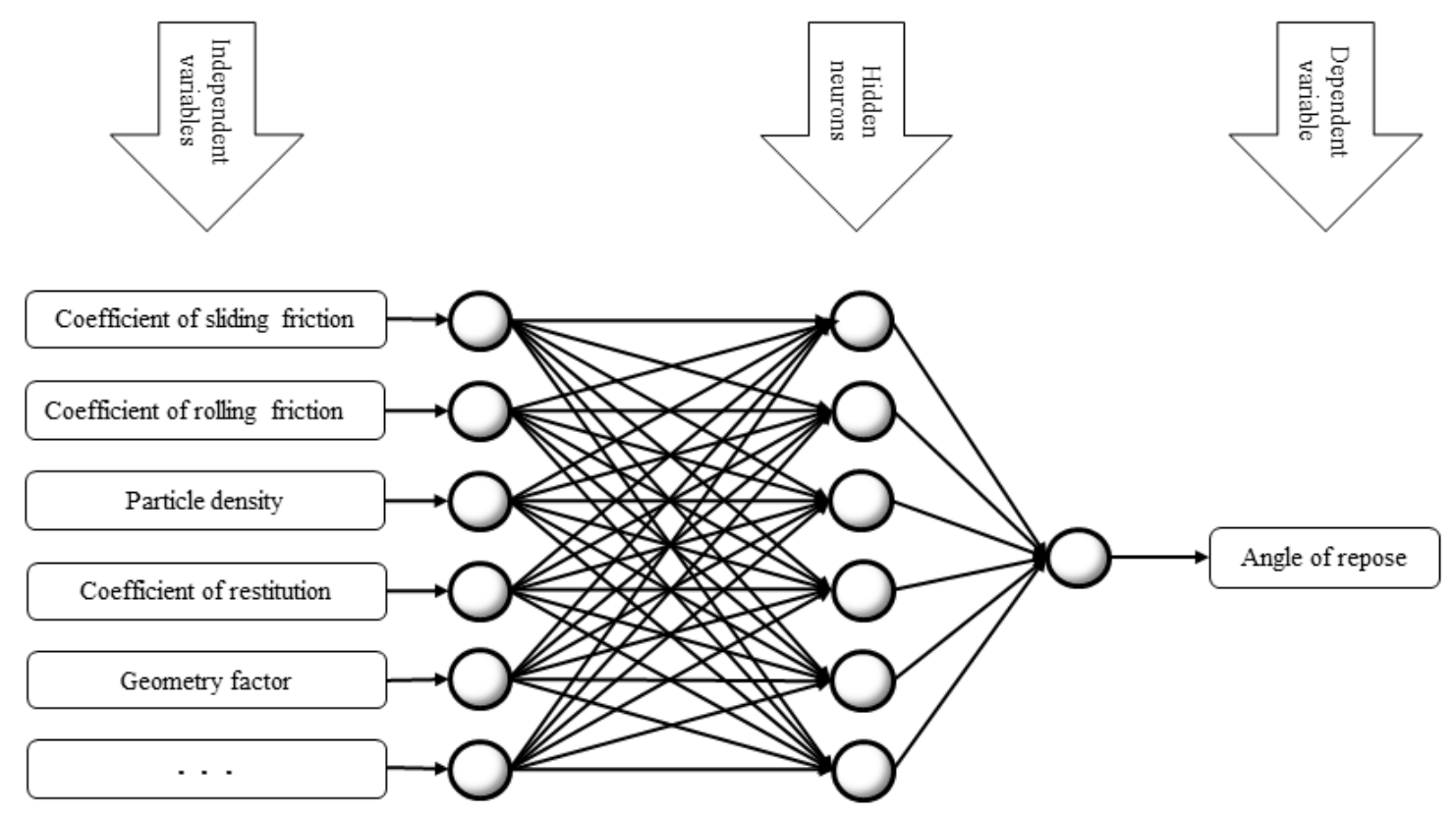

Figure 8.2: Artificial Neural Network (ANN) Scheme. Regressors are show in the left-hand side of the image. An example of a response is shown in the right-hand side of the image.

the ANN values, which demonstrates the accurate predictive power of the ANN compared to the other two methods. Increasing the number of neurons did not improve the $R^{2}$, it even started to oscillate with higher numbers of neurons. The weights and biases, which represent the trained network, can be be found in Table 8.2 In Fig. 8.3 the corresponding plot for the ANN with the maximum $R^{2}$ is shown. Each circle represents one of the 546 simulations.

We subsequently obtained the optimal number of neurons for all ANNs Table 8.3 shows the weights for the AoR.

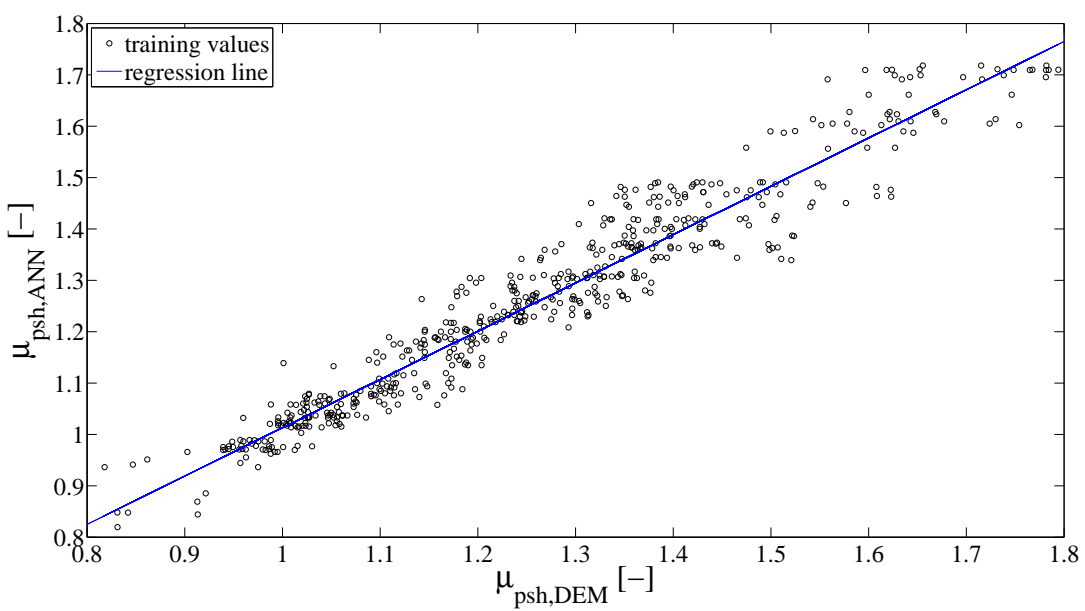

Figure 8.3: Comparison between prediction of the trained Artificial Neural Network (ANN) and 546 sinter fine full DEM simulations of the coefficient of pre-shear $\left(\overline{\mu_{p s h}}\right)$. 


\begin{tabular}{|c|c|c|c|c|c|c|c|c|c|c|}
\hline \multirow[b]{2}{*}{ Neurons } & \multicolumn{10}{|c|}{ Weights of connection between input and hidden layer } \\
\hline & 1 & 2 & 3 & 4 & 5 & 6 & 7 & 8 & 9 & 10 \\
\hline & 1.583 & -0.543 & -0.135 & -0.462 & 1.370 & 1.048 & 1.025 & -0.542 & -0.162 & -0.499 \\
\hline & -0.062 & -0.576 & -0.107 & -1.246 & -0.362 & -0.239 & 1.078 & 1.156 & 0.161 & 0.919 \\
\hline & -0.057 & 0.265 & 0.552 & -0.660 & -0.006 & 1.021 & 0.139 & -0.415 & 1.115 & 0.920 \\
\hline & 1.618 & 1.564 & 0.398 & 0.693 & 0.865 & -0.047 & 0.535 & -0.450 & -0.558 & -1.034 \\
\hline & 0.341 & -0.028 & -2.021 & -1.331 & 0.899 & -0.052 & 1.293 & 0.258 & -1.550 & 0.786 \\
\hline & 0.309 & 0.682 & 0.277 & -0.250 & 0.885 & 1.141 & 0.587 & -0.825 & -0.497 & -0.054 \\
\hline & -0.186 & 0.950 & -0.373 & -0.218 & 0.355 & -0.815 & 0.072 & 1.283 & 0.576 & -0.740 \\
\hline & \multicolumn{10}{|c|}{ Weights of connection between hidden and output layer } \\
\hline & -0.200 & -0.496 & 0.260 & -0.210 & 0.061 & 0.023 & 0.202 & 0.033 & 0.079 & 0.167 \\
\hline & \multicolumn{10}{|c|}{ Biases of hidden layer } \\
\hline & -1.906 & 2.073 & 1.657 & 1.479 & -1.280 & -1.119 & -0.770 & 0.569 & 0.345 & -0.075 \\
\hline & \multicolumn{10}{|c|}{ Weights of connection between input and hidden layer } \\
\hline \multirow[t]{14}{*}{ Neurons } & 11 & 12 & 13 & 14 & 15 & 16 & 17 & 18 & 19 & 20 \\
\hline & -0.154 & -0.126 & -0.303 & 0.798 & -1.191 & -0.704 & -0.254 & -0.988 & 0.788 & -0.612 \\
\hline & -0.292 & 0.715 & 1.266 & -0.506 & 0.074 & -1.095 & -1.565 & -1.085 & -0.968 & -0.073 \\
\hline & -0.443 & 1.142 & 0.342 & -0.198 & 0.545 & -0.202 & 0.722 & -0.577 & -1.573 & -0.440 \\
\hline & 0.918 & 0.458 & -1.031 & -0.916 & -0.092 & 0.312 & 0.935 & -0.390 & -0.489 & -1.510 \\
\hline & -0.612 & 0.922 & 1.412 & -0.305 & -0.120 & -0.696 & -0.396 & 0.834 & 0.908 & -0.022 \\
\hline & -1.047 & -1.113 & 0.228 & 1.605 & -0.899 & -1.168 & -0.017 & -0.081 & 0.341 & 0.876 \\
\hline & -1.320 & 0.114 & -0.233 & -0.013 & 1.191 & 0.087 & -0.527 & 1.106 & 0.266 & 0.877 \\
\hline & \multicolumn{10}{|c|}{ Weights of connection between hidden and output layer } \\
\hline & -0.082 & 0.173 & -0.419 & 0.054 & 0.023 & -0.056 & -0.181 & -0.150 & 0.167 & -0.580 \\
\hline & \multicolumn{10}{|c|}{ Biases of hidden layer } \\
\hline & -0.231 & -0.508 & 0.435 & 0.813 & -1.221 & -1.386 & -1.356 & -1.671 & 1.700 & -2.221 \\
\hline & \multicolumn{10}{|c|}{ Biases of output layer } \\
\hline & \multicolumn{10}{|c|}{-0.543} \\
\hline
\end{tabular}

Table 8.2: Weights and biases table for the coefficient of internal friction for sinter fine.

\subsection{Statistical tools comparison}

We checked $R^{2}$, mean absolute error,

$$
M A E=\frac{\sum_{i=1}^{n}\left(x_{i}-\widehat{x}_{i}\right)}{n},
$$

mean squared error,

$$
M S E=\frac{\sum_{i=1}^{n}\left(x_{i}-\widehat{x}_{i}\right)^{2}}{n},
$$

and root mean squared error,

$$
R M S E=\sqrt{\frac{\sum_{i=1}^{n}\left(x_{i}-\widehat{x}_{i}\right)^{2}}{n}}
$$

for the Bayesian linear regression, the Gaussian nonlinear regression, and the $A N N$ regression to establish the most effective method. All were trained with the same training set. For instance, a comparison of the $R^{2}$ for the $\mu_{p s h}$ can be see in Fig. 8.4 In Fig. 8.5 a similar comparison for the AoR can be found. In fact, the check was performed for each method by comparing the DEM bulk values of the test set against the bulk values predicted by each method from the corresponding DEM input values of the test set.

Table 8.4 shows a quantitative comparison between the three methods for the $\mu_{p s h}$ 


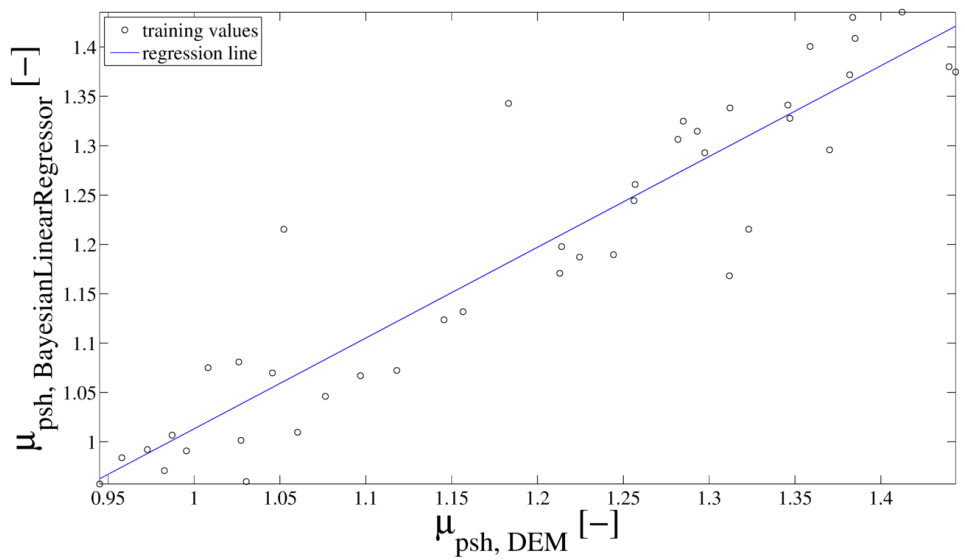

(a) SCT Bayesian linear regressor.

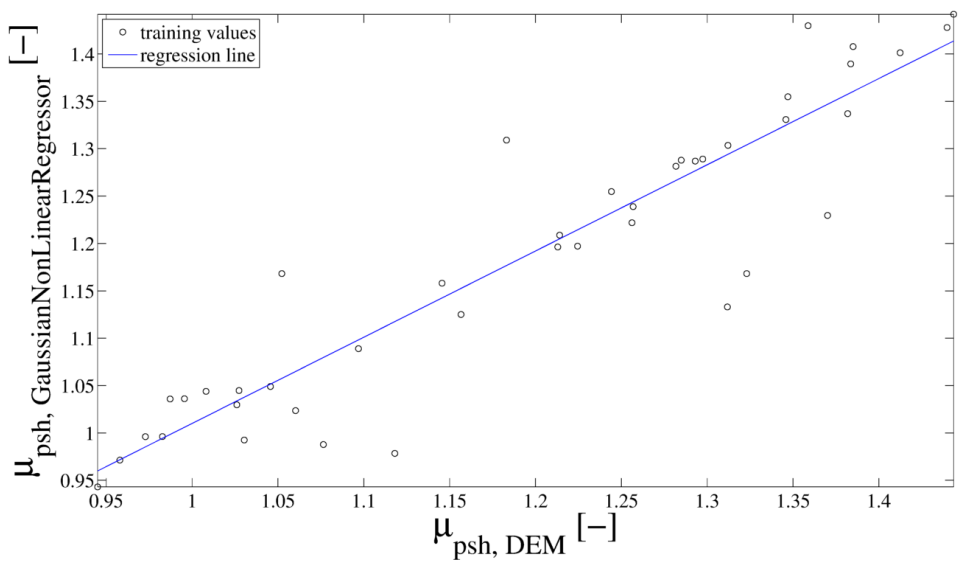

(b) SCT Gaussian non linear regressor.

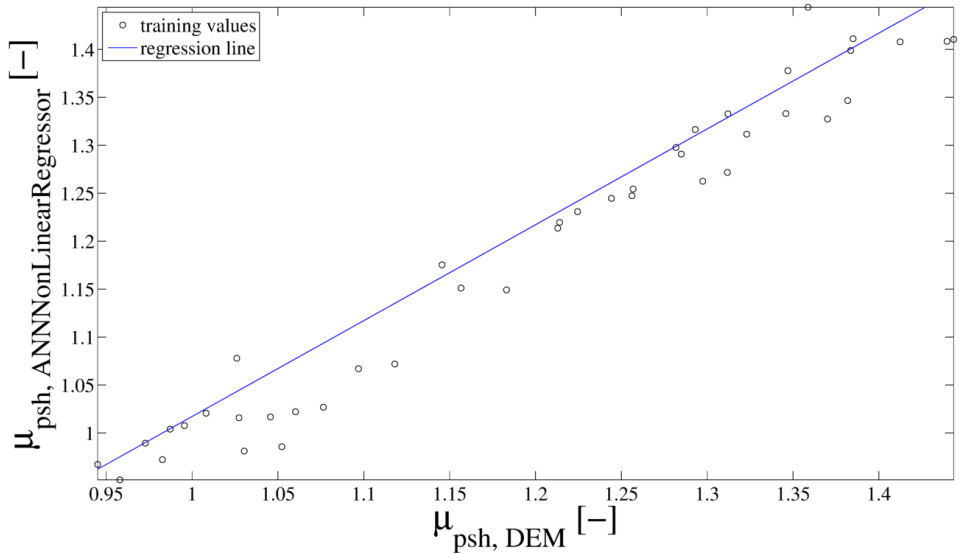

(c) SCT ANN non linear regression.

Figure 8.4: Regressions comparison for the $\mu_{p s h}$ Each point represents a simulation. In blue the regression, or interpolation, line. 


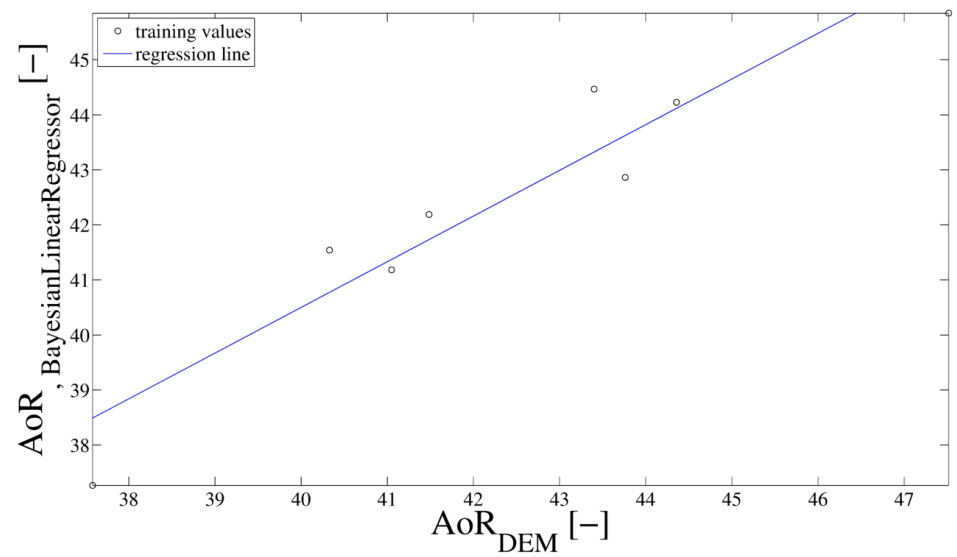

(a) AoR Bayesian linear regressor.

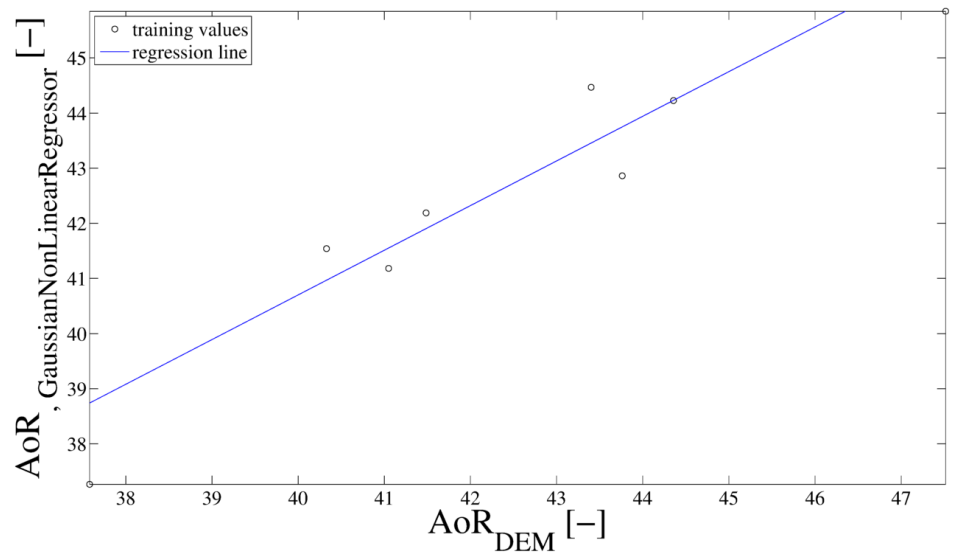

(b) AoR Gaussian non linear regressor.

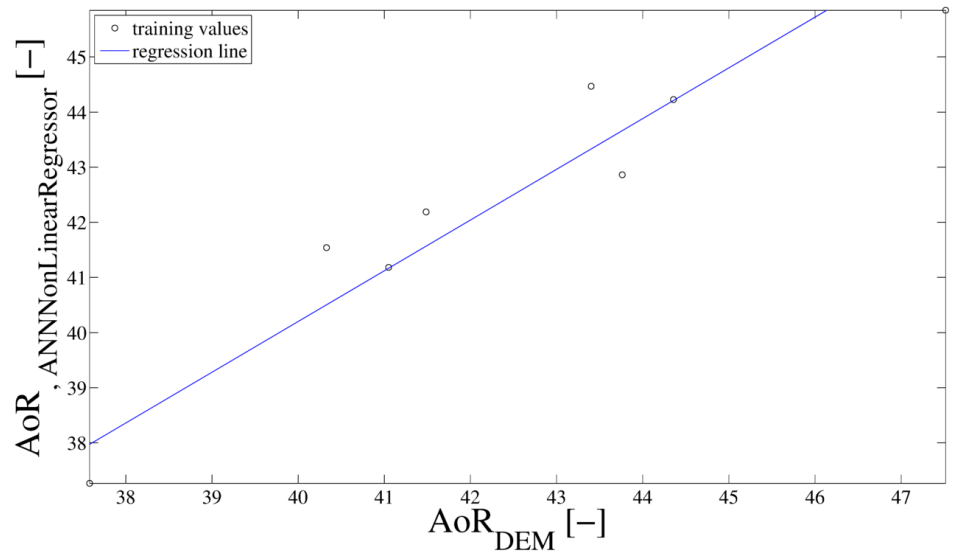

(c) AoR ANN non linear regression.

Figure 8.5: Regressions comparison for the AoR, Each point represents a simulation. In blue the regression, or interpolation, line. 


\begin{tabular}{|c|c|c|c|c|c|c|c|c|c|}
\hline \multirow[b]{2}{*}{ Neurons } & \multicolumn{9}{|c|}{ Weights of connection between input and hidden layer } \\
\hline & 1 & 2 & 3 & 4 & 5 & 6 & 7 & 8 & 9 \\
\hline & 0.148 & -0.064 & -0.585 & -1.247 & 1.264 & 0.108 & -0.335 & -0.950 & 0.722 \\
\hline & 1.889 & 1.334 & -0.105 & 1.016 & 1.781 & -0.652 & -1.664 & 1.497 & -0.578 \\
\hline & -0.271 & 1.221 & 1.086 & 0.777 & 0.298 & 0.984 & 0.101 & -1.466 & -0.230 \\
\hline & -1.316 & 0.385 & -1.997 & -1.531 & -1.159 & 1.877 & 1.227 & 0.948 & 1.643 \\
\hline & \multicolumn{9}{|c|}{ Weights of connection between hidden and output layer } \\
\hline & 0.137 & 0.437 & -0.575 & -0.025 & 0.109 & -0.667 & -0.184 & 0.119 & 0.806 \\
\hline & \multicolumn{9}{|c|}{ Biases of hidden layer } \\
\hline & -2.453 & 2.311 & 1.399 & 0.679 & 0.108 & 0.844 & -1.614 & -1.674 & 2.654 \\
\hline & \multicolumn{9}{|c|}{ Biases of output layer } \\
\hline
\end{tabular}

Table 8.3: Weights and biases table for sinter fine AoR.

\begin{tabular}{lccc}
\hline & Bayesian & Gaussian & ANN \\
\hline Coefficient of determination $\left(\underline{R^{2}}\right)$ & 0.860 & 0.843 & 0.959 \\
Mean absolute error & 0.042 & 0.038 & 0.025 \\
Mean squared error & 0.003 & 0.004 & 0.001 \\
Root mean squared error & 0.057 & 0.061 & 0.031 \\
\hline
\end{tabular}

Table 8.4: Regression methods quantitative comparison.

\subsection{Parameter Identification}

Since $\mu_{p s h} \mu_{s h}$ and $\rho_{b}$ belonged to the shear-cell simulations, their ANNs were handled together: we had one cluster with three ANNS for the shear cell and one with only one ANN for the AoR We could then proceed in identifying valid input parameters.

\subsubsection{Computational Condiderations}

Grossman and Vecchio [57], Oberkampf and Roy [106] suggested using a Design of Experiments (DoE) method to determine the parameter combinations to be simulated. They stated that this approach allows optimization of computation time with an acceptable loss of precision. The speed of the trained ANNS enabled us to follow a different approach to maximizing the precision of the characterization.

\begin{tabular}{lcccc}
\hline & $\mu_{s}$ & $\mu_{r}$ & $C o R$ & {$\left[\rho_{p}\right.$} \\
& {$[-]$} & {$[-]$} & {$[-]$} & {$[k g / m 3]$} \\
\hline range & {$[0.1 \ldots 1.0]$} & {$[0.1 \ldots 1.0]$} & {$[0.5 \ldots 0.9]$} & {$[2000 \ldots 3500]$} \\
$\#$ rnd & 100 & 100 & 25 & 25 \\
\hline
\end{tabular}

Table 8.5: DEM random input values. Within each range \# random values are chosen.

\subsubsection{Decisional Limits}

We created random values in the range and numbers defined in Table 8.5 according to a standard uniform distribution. These combinations were then fed to and processed by the selected ANNs and thus three bulk values for the shear cell and one for the AoR were obtained. The ANN evaluation was significantly faster than the DEM simulations. The individuation of the numerical bulk behaviours for all the DEM combinations did not take more than a few seconds on a single core. 
As can be seen in Fig. 8.6 in the parameters identification phase (solid lines) we identify valid input

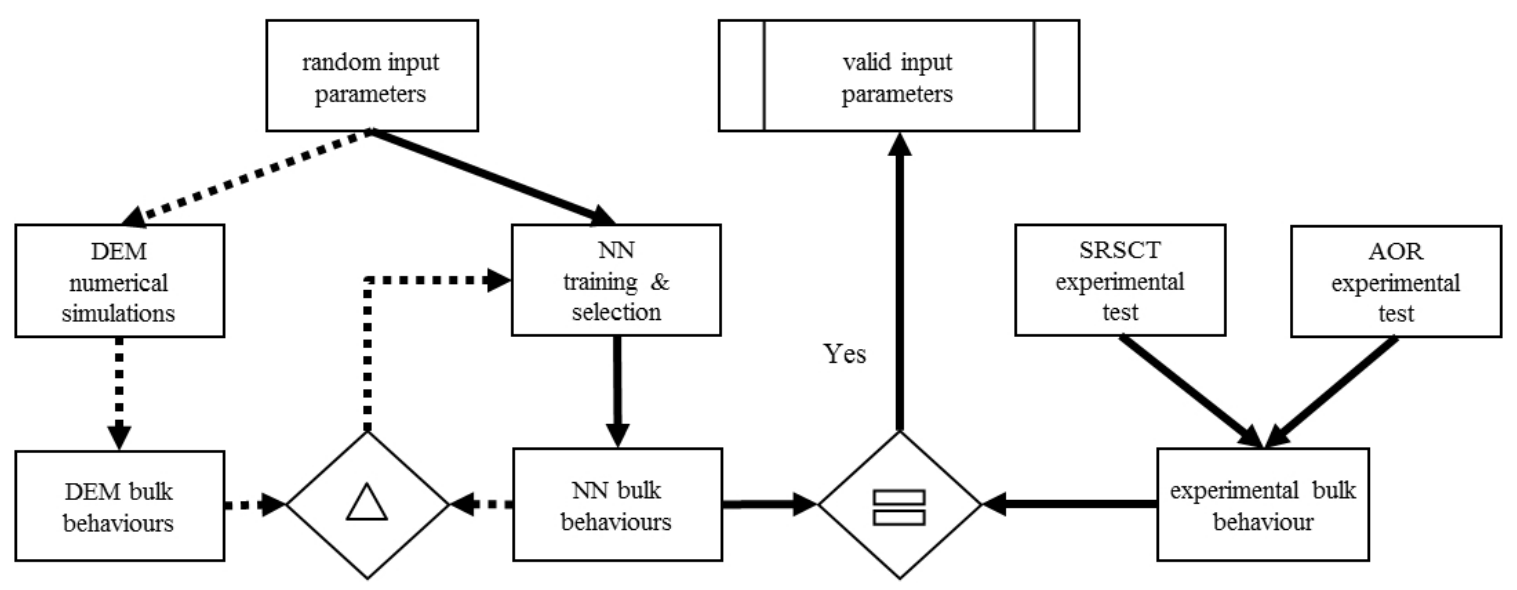

Figure 8.6: Method. In the training phase (dashed lines) DEM simulations are performed with random initial input parameters. The behaviours obtained are used to train the Artificial Neural Networks $(A N N s)$ in a loop that continues until the difference between the outputs of each $A N N$ and its simulations is below the limit $(\Delta)$. In the parameters identification phase (solid lines) we identify valid input parameters by comparing $(=)$ ANNs and experimental behaviours.

parameters by comparing $(=)$ ANNS and experimental behaviours.

We obtained for each of the twelve load conditions of the SCT three bulk values $\left(\mu_{p s h} \mu_{s h}\right.$ and $\left.\rho_{b}\right)$. The fourth bulk value was the result of two angle of repose (AoR tests that recreated the repose angle observed in a pile of the real material. Subsequently, we compared the ANN and experimental bulk behaviours for the twelve shear-cell load conditions. If in a DEM-parameter combination all the three bulk values differed by less than $5 \%$ from those of the corresponding experiments, i.e.:

$$
\begin{cases}\text { if } & \left|1-\frac{\mu_{p s h, \text { num }}}{\mu_{\text {psh,exp }}}\right|<5 \%, \\ \text { and if } & \left|1-\frac{\mu_{\text {sh,num }}}{\mu_{\text {sh,exp }}}\right|<5 \%, \\ \text { and if } & \left|1-\frac{\rho_{p, \text { num }}}{\rho_{p, \text { exp }}}\right|<5 \%,\end{cases}
$$

the combination was marked. The marked combinations were processed by the AoR ANN, and then compared with the experiment. Were considered valid those that differed by less than $5 \%$ also in this comparison (Eq. 8.6):

$$
\text { if } \quad\left|1-\frac{A o R_{\text {num }}}{A o R_{\text {exp }}}\right|<5 \% \text {. }
$$

\subsection{Sinter fine Characterization}

We realized 6,250,000 parameter of combinations random values for sinter fine. Amongst them we searched for values complying with the experimental results. Further, we investigated the reliability of the procedure, see Section 8.5.4

The comparison between numerical and experimental behaviours led to a first series of marked combinations $(M C 1)$ for the load conditions of the shear cell $\sigma_{n}=10,070 \mathrm{~Pa}, \mathrm{P}=1.0$, as plotted in Fig. 8.7b We decided to show the valid values achieved through the procedure for every material analyzed with three compact graphical schemes:

- parameter space plot,

- box plot, 
- density plot.

In the following sections, the plots regarding the sinter fine with $\sigma_{n}=10,070 \mathrm{~Pa}$ are discussed and interpreted as pattern for each material in each load condition.

\subsubsection{Parameter space plot}

An example of a parameter space plot can be seen in Fig. 8.7b. On the axes we can see:

- the coefficient of restitution (-) (CoR),

- the coefficient of sliding friction $(-)\left(\underline{\mu_{s}}\right)$,

- the coefficient of rolling friction (-) $\left(\underline{\mu_{r}}\right)$,

- the bulk density $\left(\mathrm{kg} / \mathrm{m}^{3}\right)(\underline{\rho b})$.

Further, are shown:

- the minimum input values amongst the millions possible combinations, with a blue straight line;

- the minimum values amongst the valid, or marked combinations, with a green straight line;

- the mean values amongst the valid, or marked combinations, with an orange dotted line;

- the negative standard deviations from the mean values amongst the valid, or marked combinations, with a red straight line;

- the positive standard deviations from the mean values amongst the valid, or marked combinations, with a red straight line;

- the maximum values amongst the valid, or marked combinations, with a green straight line;

- the maximum input values amongst the millions possible combinations, with a blue straight line.

The shaded area indicates valid parameter combinations, and dark shaded values indicate the confidence range. As expected, the two shaded areas cover the majority of the plot, proving at the same time 1) the non-unicity of the solution for the characterization problem and 2) the possible solutions may largerly vary over the domain.

However, the confidence range gives more punctual information. Note that the confidence interval is large, especially for the $C o R$ which highlights its insignificant influence on the characterization. Both the $\rho_{p}$ and the $\mu_{s}$, however, show a narrow confidence interval, which demonstrates their influence and the ability of this procedure to find valid DEM parameters. Further, the mean values for the $\mu_{s}$ and the $\mu_{r}$ are comparable to the highly friction resistant materials described in the literature [133. These results agree with our examination of the ratio of the standard deviation to the range, see Table 8.6 Finally, this plot is especially interesting for reliability consideration, see Section 8.5.4

\subsubsection{Box plot}

An example of a box plot can be seen in Fig. 8.8b Benjamini [16] defines a box plot a graphical representation of collections of numerical data by means of their quartiles. These are three points, which divide the data in four groups that are equals and contain a quarter of the data each.

In a box plot the values between the first quartile (25\%) and the third quartile (75\%) are included in a blue box. Further, so called baffles or whishers are lines, which are extended as far as the minimum and maximum values not considered outliers. The data which exceed the whishers are represented with red crosses. Finally, the median is represented as a red straight line. Values are normalized, meaning that for each input value the maximum extracted is one. 


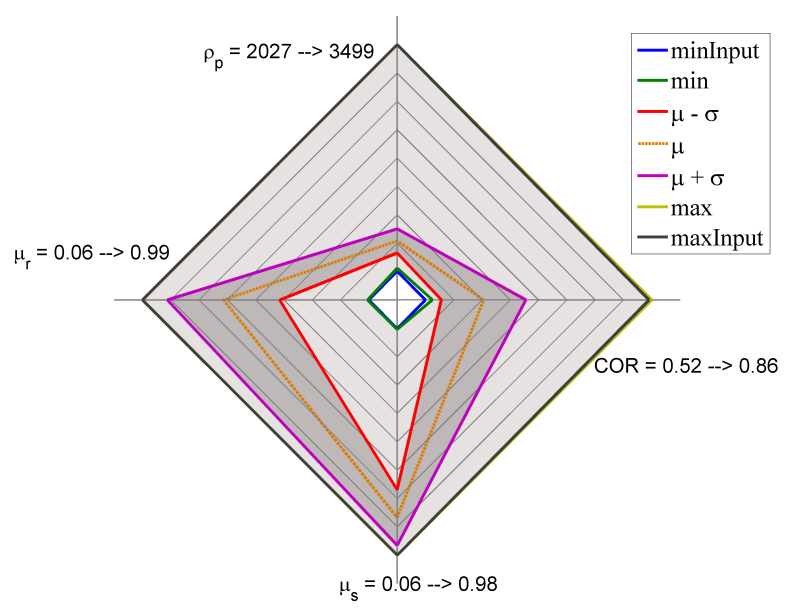

(a) Parameter space plot, $\mathrm{SCT} \sigma_{n}=10070 \mathrm{~Pa}, \mathrm{P}=0.8$.

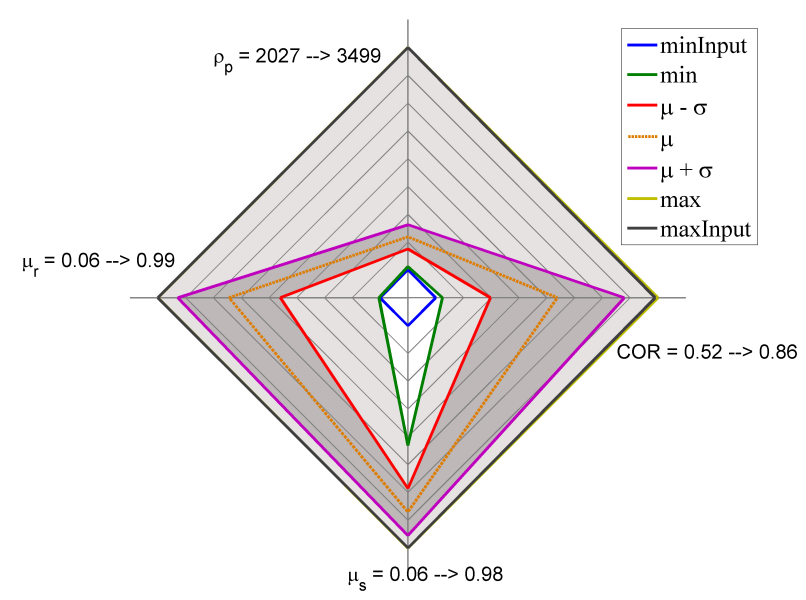

(b) Parameter space plot, SCT $\sigma_{n}=10070 \mathrm{~Pa}, \mathrm{P}=1.0$.

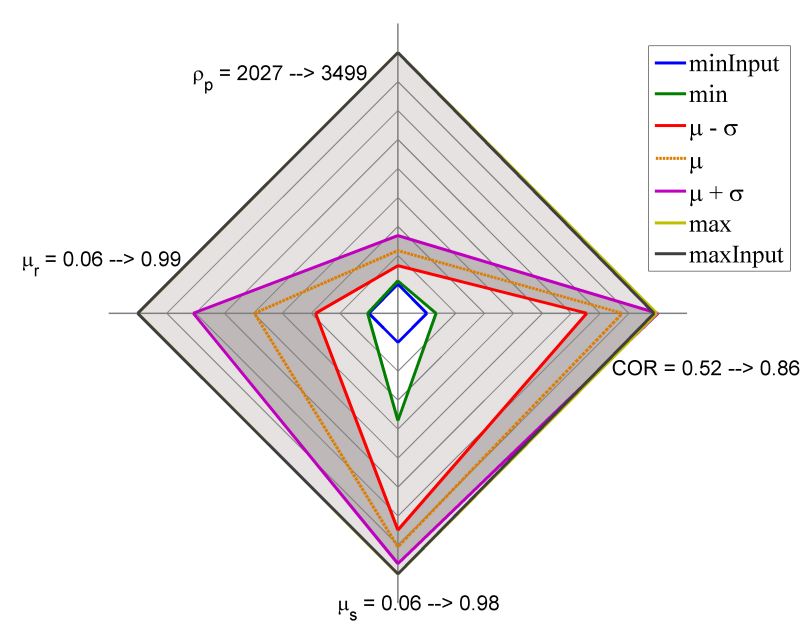

(c) Parameter space plot, $\mathrm{SCT} \sigma_{n}=10070 \mathrm{~Pa}, \mathrm{P}=1.2$.

Figure 8.7: SCT parameter space plots, $\sigma_{n}=10070 \mathrm{~Pa}$. The valid parameters are plotted over four axes, accounting for average and standard deviation. 
The representation through box plots in Fig. $8.8 \mathrm{~b}$ is consistent for the the $\rho_{p}$ and the $\mu_{s}$ with those shown in the parameter space plot in Fig. 8.7b However, the box plot representation helps to underline the much higher level of variability for the $\mu_{r}$ compared to the $C o R$. This can be explained if we consider again the non-unicity of the solution and how the $\mu_{r}$ has a reduced influence on the shear cell results compared to the $\mu_{s}$. This aspect is even clear in a density plot.

\subsubsection{Density plot}

Further, we observed that various DEM parameter combinations could reproduce the experimental behaviour, and thus evaluated their mutual dependencies. This is shown more clearly in a density plot (e.g., Fig. 8.9a of the particles' coefficient of restitution (CoR) in relation to the coefficients of sliding friction $\left(\underline{\mu_{s}}\right)$ and rolling friction $\left(\underline{\mu_{r}}\right)$. The $\rho_{p}$ was not considered, since every valid combination has the same value for it (or a close number).

In the white area, no valid sets of simulation parameters could be found. In each cell the valid sets are grouped according to the 4 different COR ranges. Each cell is coloured according to the group with the most members.

It is clear their correlation, as already stated by Wensrich and Katterfeld [139. For high values of the $C o R$ small values for $\mu_{s}$ and $\mu_{r}$ are sufficient to reach the desired frictional bulk values. On the other hand, with low $C o R$ values, high numbers for the friction micro coefficients are necessary to achieve the same results. This effect agrees with the contact law used, described in Chapter 4

For the $\sigma_{n}=10,070 \mathrm{~Pa}, \mathrm{P}=1.0$ case, Fig. $8.9 \mathrm{~b}$. multiple combinations $\left(250,407\right.$ or $4 \%$ of the total) of $\mu_{s}$ and $\underline{\mu_{r}}$ reproduced the experimental behaviour with varying $C_{0} R$

\subsubsection{Reliability Considerations}

We tested the marked combinations by modifying the experimental bulk values of the shear cell to further prove the validity of the system. We artificially decreased or increased the shear force, and thus $\mu_{p s h}$ and $\mu_{s h}$ by a product coefficient $(P)$, e.g. Eq. 8.7 .

$$
\mu_{p s h, \text { new }}=\mu_{\text {psh,old }} \cdot P .
$$

First, we set it to $P=0.8$, and we obtained another series of marked combinations $(M C 2)$. It could be seen in the parameter space plot in Fig. 8.7a that the confidence range is narrower than for $P=1.0$, while in the density plot in Fig. $8.9 \mathrm{a}$ the area appears larger, although slightly less densely populated. Finally, for $P=1.2$ and its marked combinations $(M C 3)$ the parameter space plot in Fig. $8.7 \mathrm{C}$ shows a largely different confidence range, while the density plot in Fig. $8.9 \mathrm{c}$ shows a smaller area. As expected, the procedure was highly sensitive to variations in the experimental data. Our approach could therefore be used for a wide range of bulk materials.

\subsubsection{AoR results}

We then processed the random combinations with the AoR ANN In Fig. 8.10a the parameter space plot for the same criteria as before could be seen. In accordance with theory (Wensrich and Katterfeld [139]), in a simulation dominated by rolling particles, the coefficient of rolling friction has the maximum influence.

\subsubsection{Merge results}

Finally, we extracted from the $M C 1$ values the AoR ANN behaviour and compared it with the experimental one. As could be seen in the parameter space plot in Fig. 8.11. the confidence interval is very small, indicating that all the parameters but the $C o R$ played an important role, and demonstrating the 


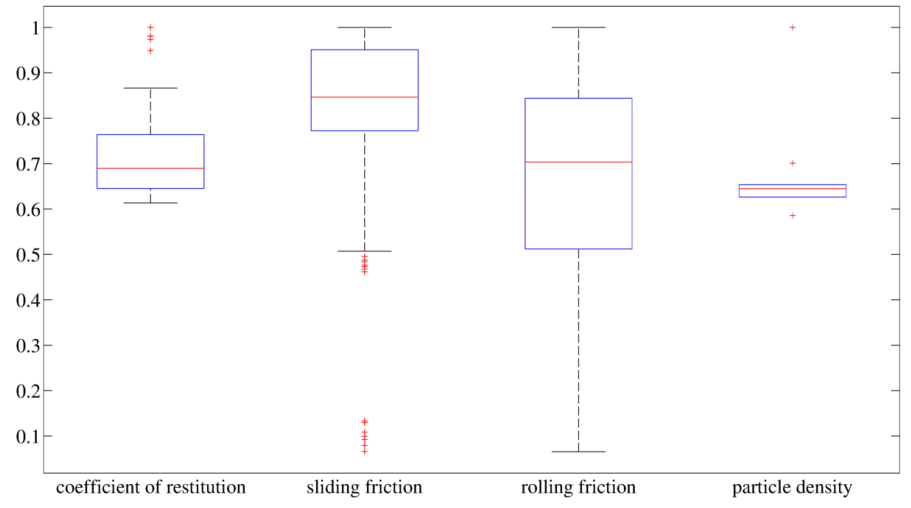

(a) Box plot, $\underline{\mathrm{SCT}} \sigma_{n}=10070 \mathrm{~Pa}, \mathrm{P}=0.8$

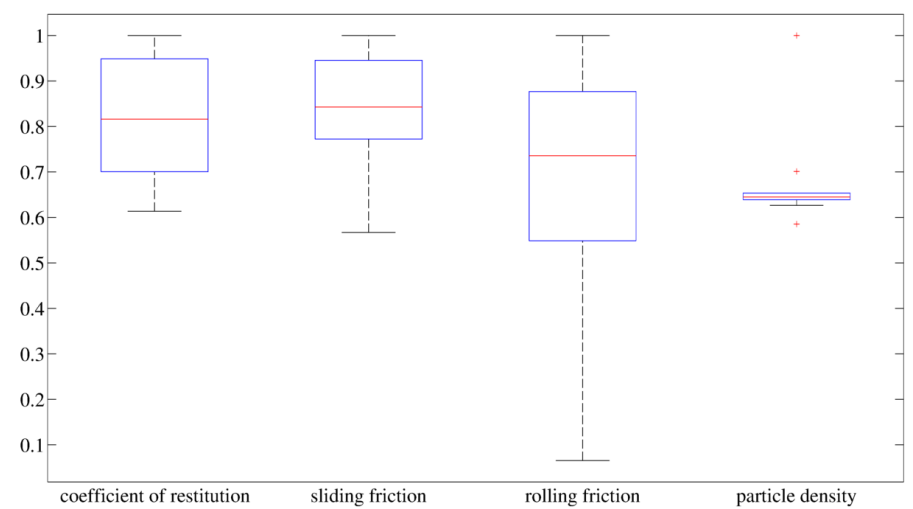

(b) Box plot, $\mathrm{SCT} \sigma_{n}=10070 \mathrm{~Pa}, \mathrm{P}=1.0$.

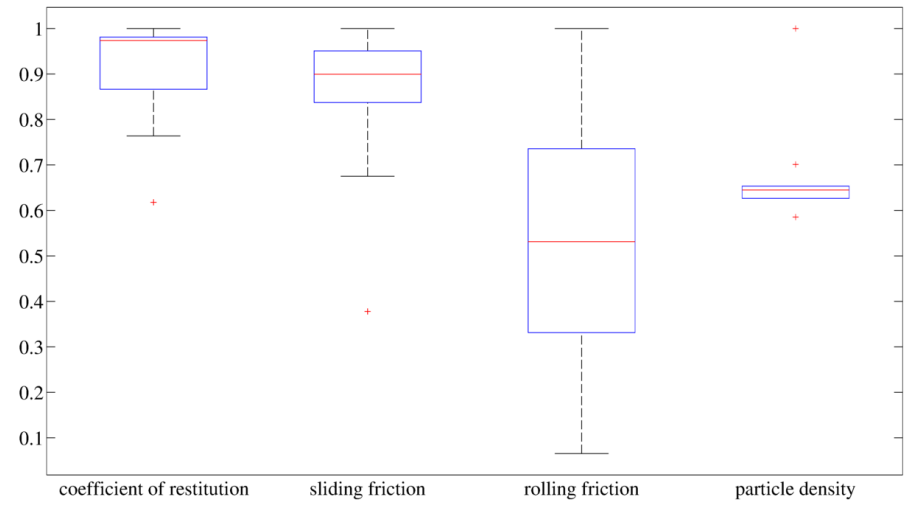

(c) Box plot, $\mathrm{SCT} \sigma_{n}=10070 \mathrm{~Pa}, \mathrm{P}=1.2$.

Figure 8.8: SCT box plots for sinter fine, $\sigma_{n}=10070 \mathrm{~Pa}$. The range of valid parameters is shown, together with the average and the 25 and 75 percentile. 


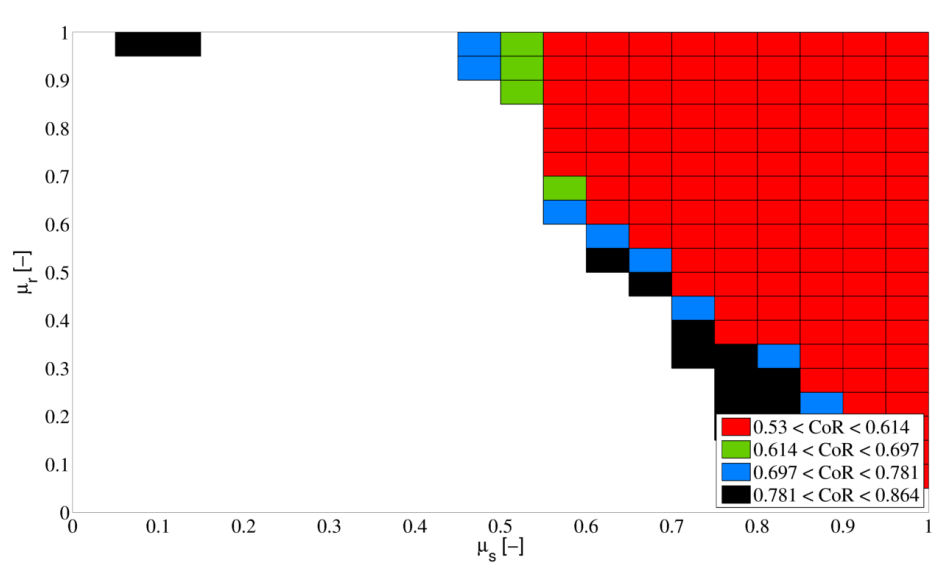

(a) Density plot, $\underline{\mathrm{SCT}} \sigma_{n}=10070 \mathrm{~Pa}, \mathrm{P}=0.8$.

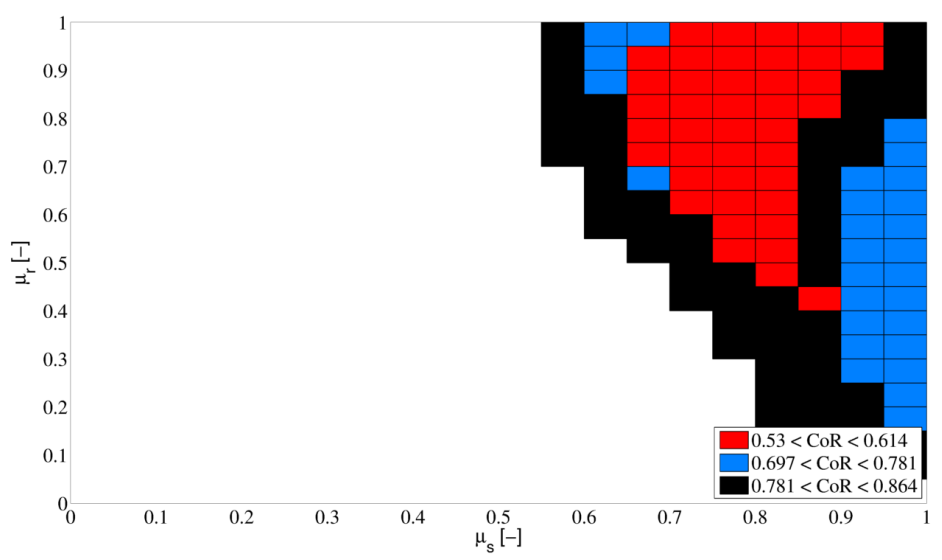

(b) Density plot, SCT $\sigma_{n}=10070 \mathrm{~Pa}, \mathrm{P}=1.0$.

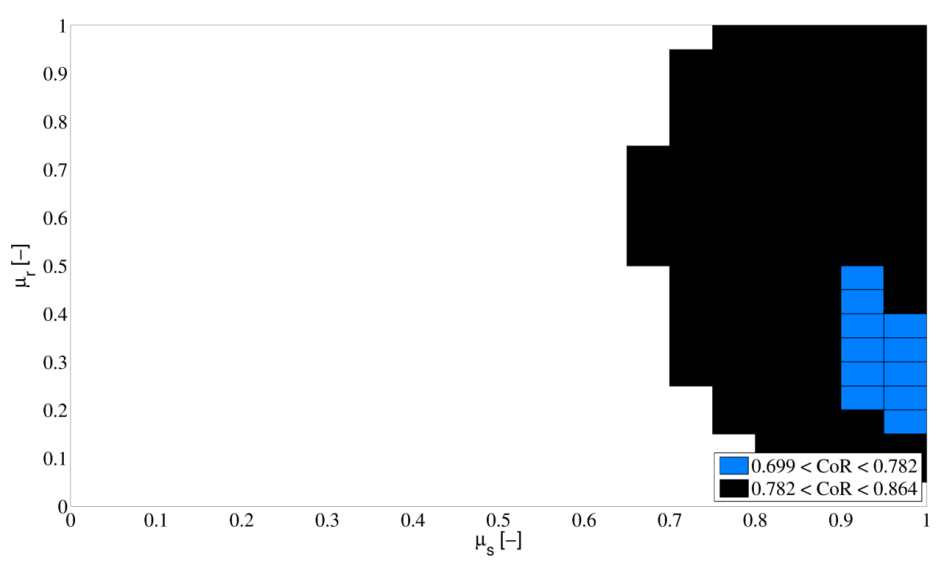

(c) Density plot, $\underline{\mathrm{SCT}} \sigma_{n}=10070 \mathrm{~Pa}, \mathrm{P}=1.2$.

Figure 8.9: Density plot comparison of SCT results. The $\mu_{s}$ and $\mu_{r}$ valid parameters are plotted over two axes, and coloured according to the $C o R$ 


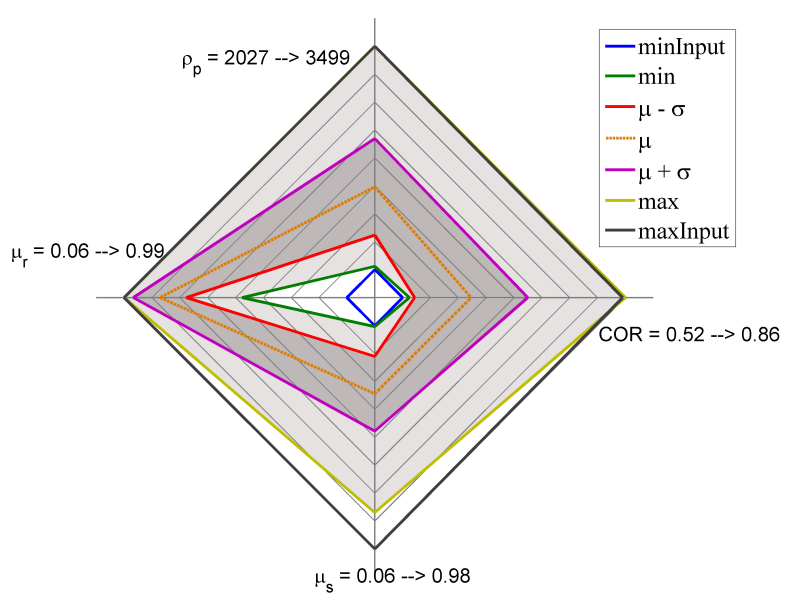

(a) Parameter space plot, $A o R_{\exp }=38.85^{\circ}$.

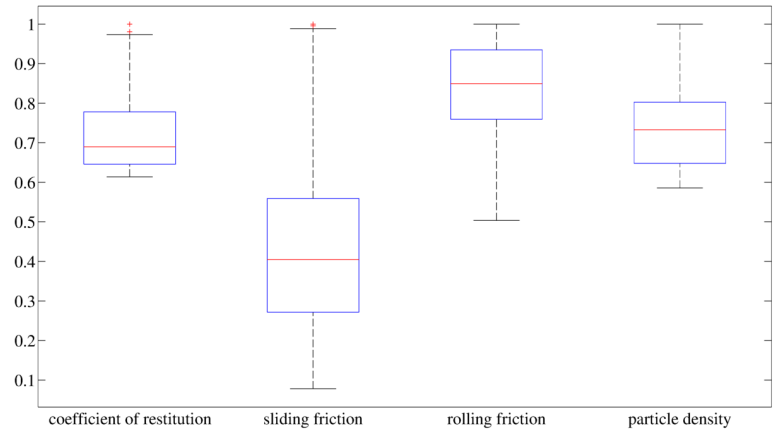

(b) Box plot, $A o R_{\exp }=38.85^{\circ}$.

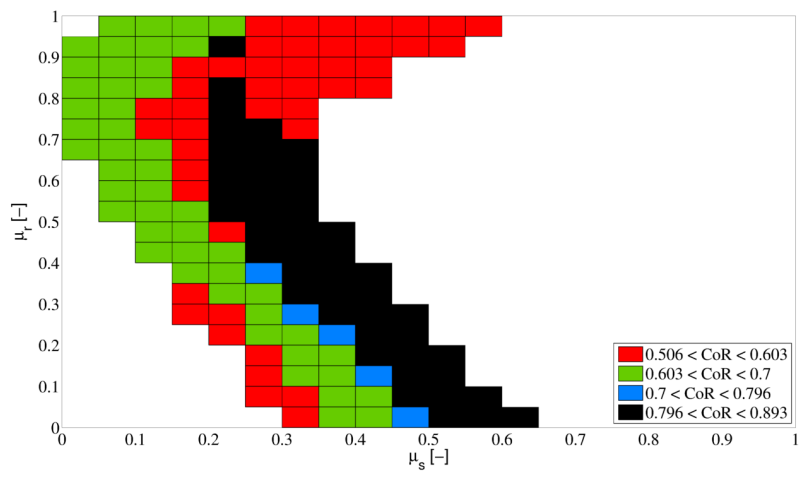

(c) Density plot plot, $A o R_{\exp }=38.85^{\circ}$.

Figure 8.10: $\mathrm{AoR}$ valid values plots. The valid values for the $\mathrm{AoR}$ sinter fine test are shown in three different plots. The results are clearly different from the $S \mathrm{SCT}$ test and the $\mu_{r}$ is the most relevant parameter. 


\begin{tabular}{ll|ccc}
\hline & & SSC & AoR & SSC \& AoR \\
& $\sigma_{n}[\mathrm{~Pa}]$ & 10000 & & \\
\hline$\mu_{s}$ & mean & 0.831 & 0.177 & 0.664 \\
& std. dev. (SD) & 0.097 & 0.095 & 0.029 \\
& range $\left(R_{i}\right)$ & 0.9 & 0.9 & 0.9 \\
& SD / R & 0.108 & 0.106 & 0.032 \\
\hline$\mu_{r}$ & mean & 0.692 & 0.830 & 0.916 \\
$(-)$ & std. dev. (SD) & 0.215 & 0.193 & 0.042 \\
& range $\left(R_{i}\right)$ & 0.9 & 0.9 & 0.9 \\
& SD / R & 0.239 & 0.214 & 0.046 \\
\hline COR & mean & 0.708 & 0.590 & 0.590 \\
$(-)$ & std. dev. (SD) & 0.104 & 0.073 & 0.065 \\
& range $\left(R_{i}\right)$ & 0.4 & 0.4 & 0.4 \\
& SD / R & 0.259 & 0.183 & 0.161 \\
\hline$\rho_{p}$ & mean & 2245.7 & 3192.8 & 2283.9 \\
$\left(k g / m^{3}\right)$ & std. dev. (SD) & 80.5 & 277.4 & 67.1 \\
& range $\left(R_{i}\right)$ & 1500 & 1500 & 1500 \\
& SD / R & 0.054 & 0.185 & 0.045 \\
\hline valid & number & 290203 & 816552 & 3884 \\
combinations & $(\%)$ & 4.64 & 13.06 & 0.06 \\
\hline
\end{tabular}

Table 8.6: Valid DEM values for sinter fine. For each parameter we show the valid parameter statistics in the two tests and in their intersection. Finally, we show the number of valid parameter combinations over the total (6250000).

reliability of these parameter combinations in representing the bulk behaviour. From the initial 6,250,000 combinations, only 3,884 were valid $(0.0621 \%)$, see Table 8.6

\subsection{Remaining Materials Characterization}

We later proceeded in further expanding the characterization by investigating the values for the remaining materials. Thus, we realized a limited number of simulations (620 SCT simulations and 180 AoR simulations) with the particles distributions in Table 6.2 and the stress conditions in Table 6.5

Together with the simulations already performed for sinter fine, we trained additional ANNs for the four bulk values involved. Again, we extracted those with the maximum $R^{2}$ The weights and biases for the $\mu_{i e}$ can be found in Table 8.7. Similarly, weights and biases for the AoR are in Table 8.8

Given the results in Section 8.5.4 we did not deem necessary to repeat the reliability procedure for every material analyzed.

\subsubsection{Coke coarse Characterization}

The valid values identified for the coke coarse can be found in Table 8.9.

However, while the plots referring to the single test show reasonably narrow confidence ranges, Fig. $8.12 \mathrm{e}$ shows unreasonably large valid ranges. For comparison, in Fig. 8.12f only a tiny portion of the density plot is colored. In fact, this means that the choice of valid values is limited, as Table 8.9 shows and as we expected. The use of two different plots is once more justified.

\subsubsection{Coke fine Characterization}

The range of valid values for coke is even narrower than that for the coke coarse, see Fig. 8.13a. The final results can be read in Table 8.10 . 


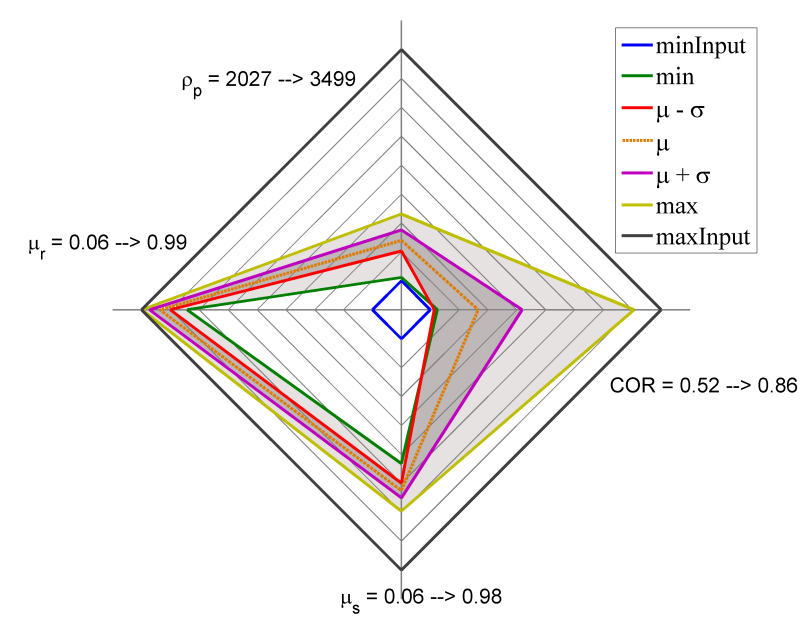

(a) Parameter space plot, $A o R_{\exp }=38.85^{\circ} \&\left[\mathrm{SCT} \sigma_{n}=10070\right.$ $\mathrm{Pa}$.

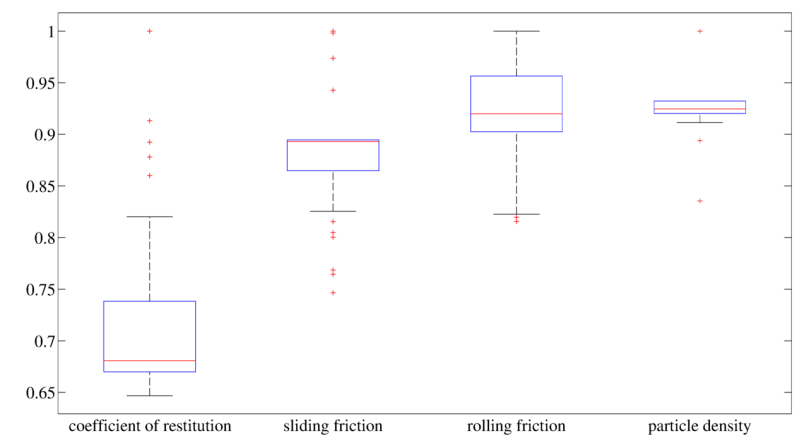

(b) Box plot, $A o R_{\text {exp }}=38.85^{\circ} \&$ SCT $\sigma_{n}=10070 \mathrm{~Pa}$.

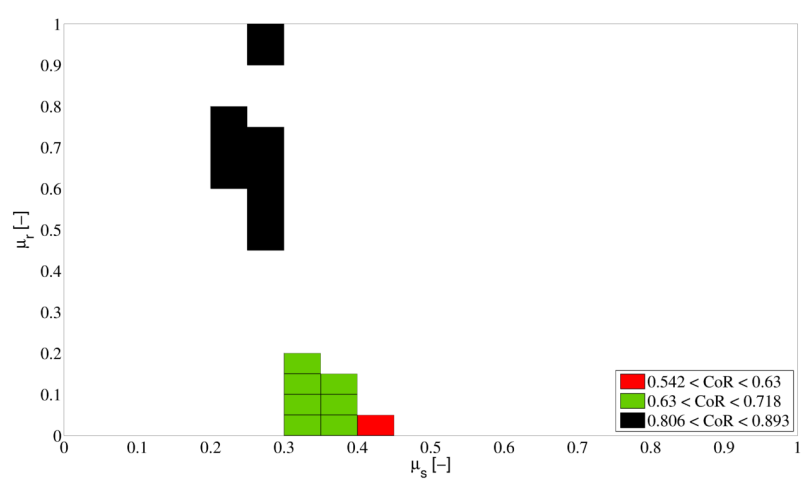

(c) Density plot plot, $A \circ R_{e x p}=38.85^{\circ} \&$ SCT $\sigma_{n}=10070 \mathrm{~Pa}$.

Figure 8.11: Merge parameter space plots of the sinter fine. A limited number of parameters are valid for both tests. It is clear that the friction coefficients are the most relevant micro parameters and only a small range of their values can be used to successfully model the bulk behaviour of the sinter fine. 


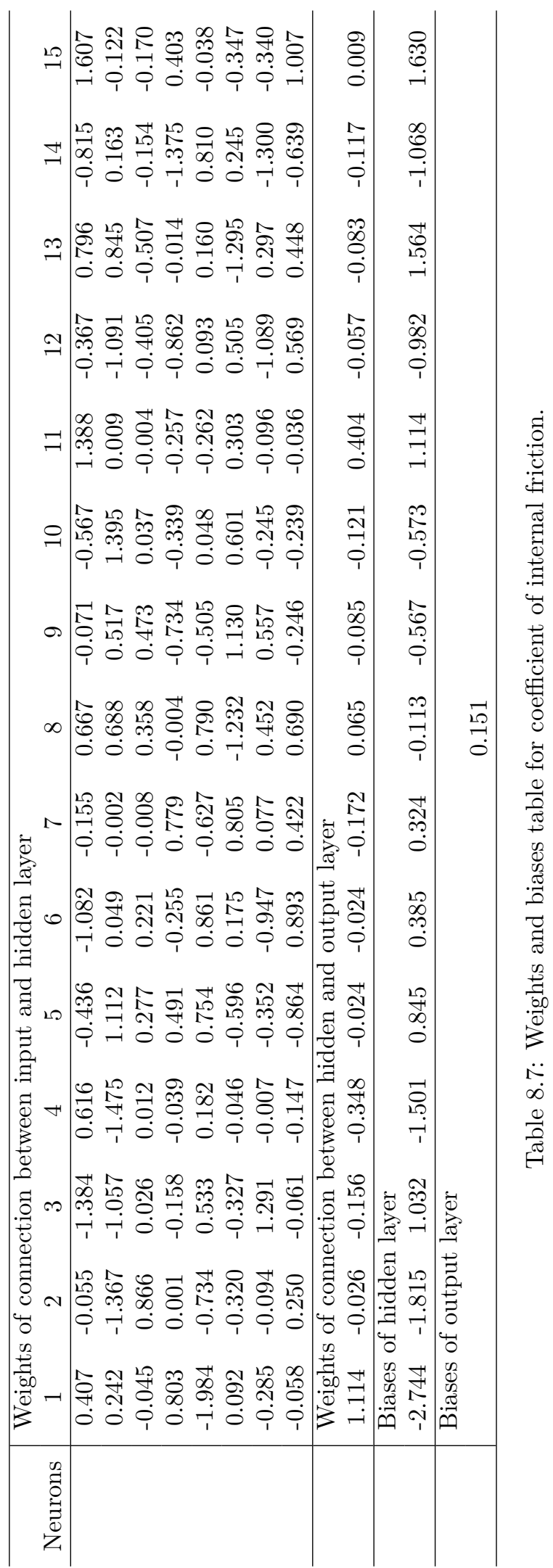




\begin{tabular}{|c|c|c|c|c|c|c|c|c|c|}
\hline \multirow[b]{2}{*}{ Neurons } & \multicolumn{9}{|c|}{ Weights of connection between input and hidden layer } \\
\hline & 1 & 2 & 3 & 4 & 5 & 6 & 7 & 8 & 9 \\
\hline & 1.312 & -1.389 & -1.470 & 0.368 & -0.259 & -0.014 & 3.356 & -0.692 & 0.446 \\
\hline & -1.729 & 0.699 & -0.660 & 0.622 & 0.769 & 1.156 & 0.488 & -0.656 & 0.601 \\
\hline & -0.539 & -1.052 & 0.228 & -0.535 & -0.180 & -0.870 & -0.149 & 0.672 & -0.721 \\
\hline & 0.029 & -0.100 & 0.357 & -1.181 & 1.490 & -0.777 & 0.016 & -0.824 & -0.620 \\
\hline & -0.022 & -0.173 & 0.597 & 1.444 & 0.736 & 1.141 & -0.029 & -1.045 & -0.859 \\
\hline & 0.192 & -0.239 & 0.320 & -0.151 & 0.357 & -0.464 & -0.042 & -0.759 & 0.735 \\
\hline & \multicolumn{9}{|c|}{ Weights of connection between hidden and output layer } \\
\hline & -0.512 & 0.242 & -0.098 & 0.030 & -0.068 & -0.003 & 0.750 & -0.013 & -0.010 \\
\hline & \multicolumn{9}{|c|}{ Biases of hidden layer } \\
\hline & -2.204 & 1.678 & 0.999 & -0.526 & -0.294 & -0.298 & 0.683 & -1.631 & 2.343 \\
\hline & \multicolumn{9}{|c|}{ Biases of output layer } \\
\hline
\end{tabular}

Table 8.8: Weights and biases table for AOR.

\begin{tabular}{ll|cccc}
\hline & & SSC & SSC & AoR & SSC \& AoR \\
& $\sigma_{n}[\mathrm{~Pa}]$ & 1000 & 2000 & & \\
\hline$\mu_{s}$ & mean & 0.611 & 0.582 & 0.541 & 0.456 \\
$(-)$ & std. dev. (SD) & 0.118 & 0.118 & 0.138 & 0.037 \\
& range $\left(R_{i}\right)$ & 0.959 & 0.959 & 0.959 & 0.959 \\
& SD / R & 0.123 & 0.123 & 0.144 & 0.038 \\
\hline$\mu_{r}$ & mean & 0.616 & 0.637 & 0.612 & 0.811 \\
$(-)$ & std. dev. (SD) & 0.231 & 0.194 & 0.263 & 0.081 \\
& range $\left(R_{i}\right)$ & 0.950 & 0.950 & 0.950 & 0.950 \\
& SD / R & 0.243 & 0.205 & 0.277 & 0.085 \\
\hline$C o R$ & mean & 0.648 & 0.553 & 0.675 & 0.647 \\
$(-)$ & std. dev. (SD) & 0.057 & 0.048 & 0.123 & 0.023 \\
& range $\left(R_{i}\right)$ & 0.387 & 0.387 & 0.387 & 0.387 \\
& SD / R & 0.146 & 0.124 & 0.317 & 0.060 \\
\hline$\rho_{p}$ & mean & 2748.1 & 2697.6 & 2747.7 & 2927.3 \\
$(-)$ & std. dev. (SD) & 413.4 & 410.0 & 409.3 & 388.4 \\
& range $\left(R_{i}\right)$ & 1394.6 & 1394.6 & 1394.6 & 1394.6 \\
& SD / R & 0.3 & 0.3 & 0.3 & 0.3 \\
\hline valid & number & 193070 & 137121 & 654785 & 5481 \\
combinations & $(\%)$ & 0.06 & 0.04 & 0.21 & 0.00 \\
\hline
\end{tabular}

Table 8.9: Valid DEM values for coke coarse. For each parameter we show the valid parameter statistics in the the tests and in their intersection. Finally, we show the number of valid parameter combinations over the total (3062500). 


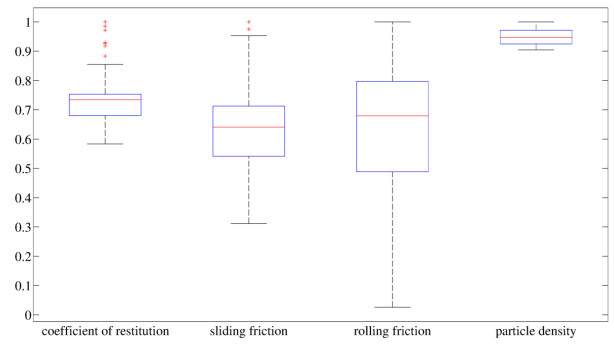

(a) Box plot SCT

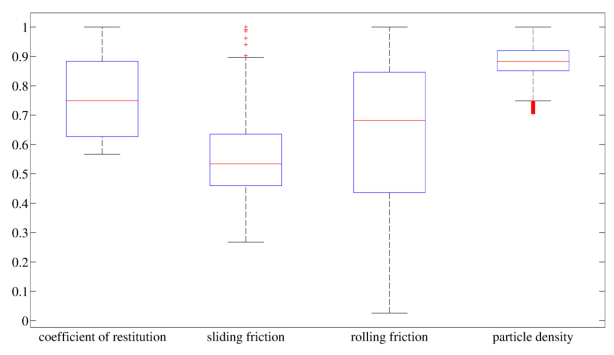

(c) Box plot AoR

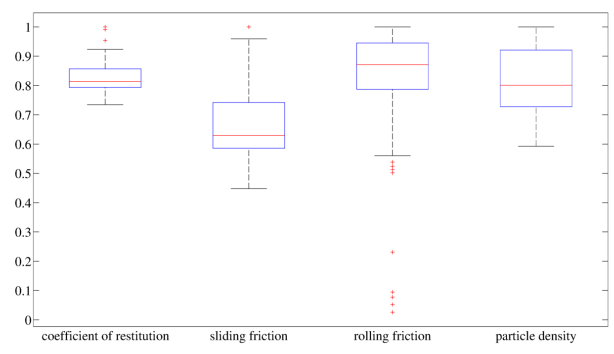

(e) Box plot intersection: $\mathrm{AoR} \& \mathrm{SCT}$

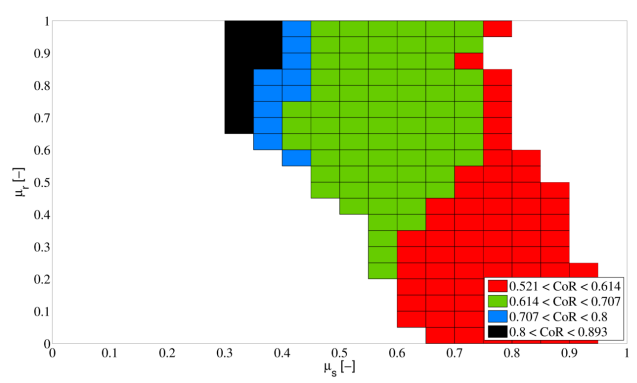

(b) Density plot $\mathrm{SCT}$

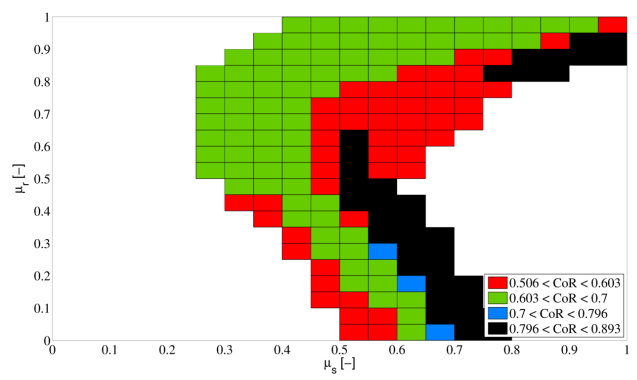

(d) Density plot AoR

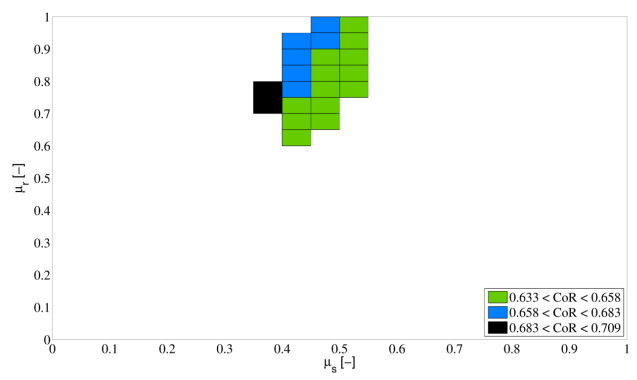

(f) Density plot intersection: AoR \& SCT

Figure 8.12: Coke coarse. The valid values for the $\mathrm{SCT}$ and $\mathrm{AoR}$ tests are shown, together with the merge values, valid for both. The plots referring to the single test show reasonably narrow confidence ranges, while Fig. $8.12 \mathrm{e}$ shows unreasonably large valid ranges. See Section 8.6 for the interpretation. 
Regrettably, considerations similar to those in Section 8.6.1 for coke coarse can be formulated for the intersation box plot. Fig. 8.13f shows the valid values.

\begin{tabular}{ll|ccccc}
\hline & & SSC & SSC & SSC & AoR & SSC \& AoR \\
& $\sigma_{n}[\mathrm{~Pa}]$ & 1000 & 2000 & 5000 & & \\
\hline$\mu_{s}$ & mean & 0.216 & 0.151 & 0.173 & 0.541 & 0.256 \\
& std. dev. (SD) & 0.028 & 0.026 & 0.023 & 0.138 & 0.017 \\
& range $\left[R_{i}\right]$ & 0.959 & 0.959 & 0.959 & 0.959 & 0.959 \\
& SD / R & 0.029 & 0.027 & 0.024 & 0.144 & 0.018 \\
\hline$\mu_{r}$ & mean & 0.310 & 0.330 & 0.229 & 0.612 & 0.535 \\
$(-)$ & std. dev. (SD) & 0.139 & 0.139 & 0.215 & 0.263 & 0.053 \\
& range $\left[R_{i}\right)$ & 0.950 & 0.950 & 0.950 & 0.950 & 0.950 \\
& SD / R & 0.146 & 0.146 & 0.226 & 0.277 & 0.056 \\
\hline$C$ CoR & mean & 0.687 & 0.689 & 0.808 & 0.675 & 0.586 \\
$(-)$ & std. dev. (SD) & 0.125 & 0.122 & 0.090 & 0.123 & 0.057 \\
& range $\left[R_{i}\right)$ & 0.387 & 0.387 & 0.387 & 0.387 & 0.387 \\
& SD / R & 0.322 & 0.314 & 0.234 & 0.317 & 0.148 \\
\hline$\rho_{p}$ & mean & 2770.6 & 2758.0 & 2358.5 & 2747.7 & 2544.5 \\
$(-)$ & std. dev. (SD) & 406.2 & 404.3 & 289.2 & 409.3 & 373.5 \\
& range $\left(R_{i}\right)$ & 1394.6 & 1394.6 & 1394.6 & 1394.6 & 1394.6 \\
& SD / R & 0.3 & 0.3 & 0.2 & 0.3 & 0.3 \\
\hline valid & number & 122110 & 136095 & 8767 & 654785 & 2718 \\
combinations & $(\%)$ & 0.04 & 0.04 & 0.00 & 0.21 & 0.00 \\
\hline
\end{tabular}

Table 8.10: Valid DEM values for coke fine. For each parameter we show the valid parameter statistics in the the tests and in their intersection. Finally, we show the number of valid parameter combinations over the total (3062500).

\subsubsection{Iron ore coarse Characterization}

The iron ore coarse is subject to the identical considerations of coke coarse, shown in Section 8.6.1 Fig. 8.14, together with Table 8.11, show the final values.

\subsubsection{Iron ore fine Characterization}

Even the iron ore fine is subject to the identical considerations of coke fine, shown in Section 8.6.2 Fig. 8.15, together with Table 8.12 show the final values.

\subsubsection{Limestone coarse Characterization}

The same effects of coke coarse and iron ore coarse are visible when investigating the limestone coarse. Fig. 8.16, together with Table 8.13, show the final values.

\subsubsection{Limestone fine Characterization}

For the limestone fine we obtained reasonable box plots, but also comsistent density plots, see Fig. 8.17 The final values can be found in Table 8.14.

\subsubsection{Sinter coarse Characterization}

The same effects of coke coarse, iron ore coarse, and limestone coarse are visible when investigating the sinter coarse. Fig. 8.18 together with Table 8.15, show the final values. 


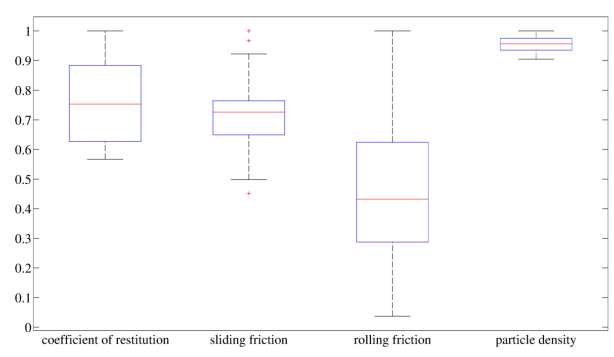

(a) Box plot SCT

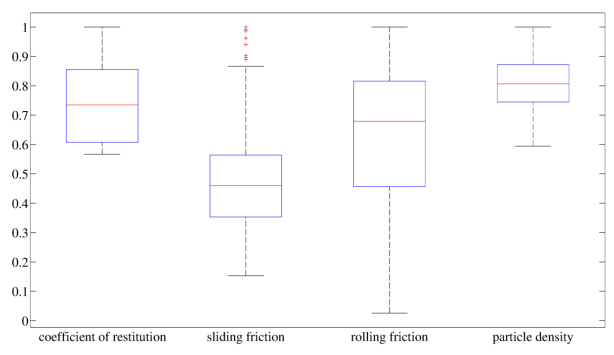

(c) Box plot AoR

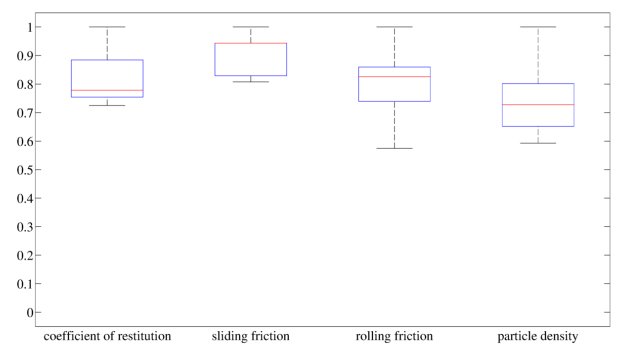

(e) Box plot intersection: $\mathrm{AoR} \& \mathrm{SCT}$

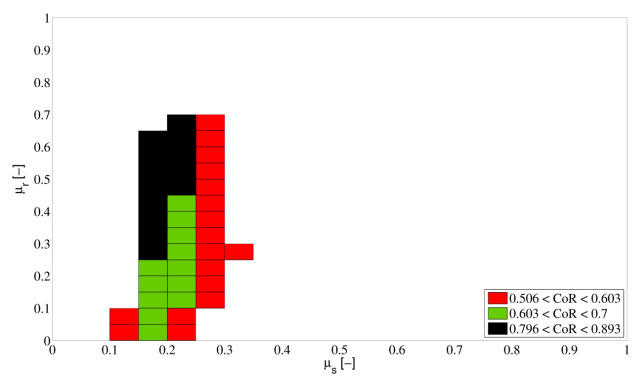

(b) Density plot $\mathrm{SCT}$

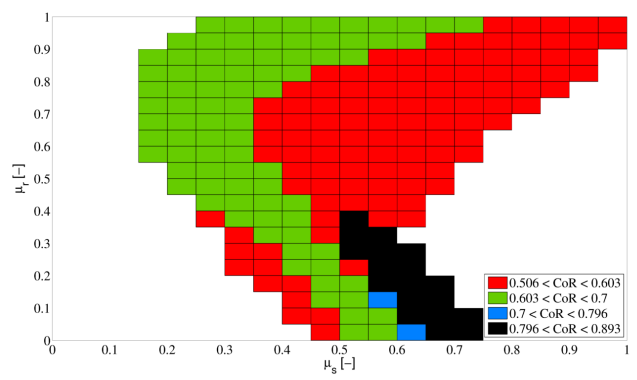

(d) Density plot AoR

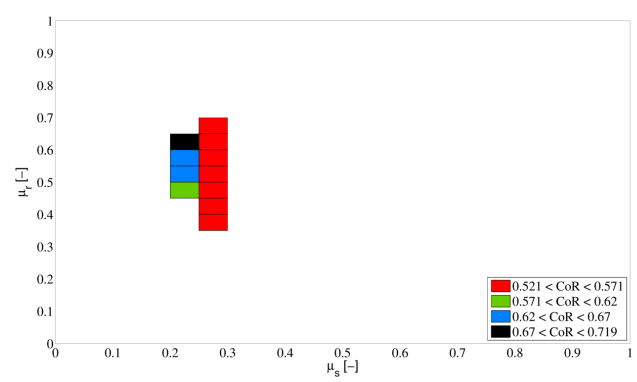

(f) Density plot intersection: AoR \& SCT

Figure 8.13: Coke fine valid values. The valid values for the $\mathrm{SCT}$ and $\mathrm{AoR}$ tests are shown, together with the merge values, valid for both. The plots referring to the single test show reasonably narrow confidence ranges, while Fig. $8.13 \mathrm{e}$ shows unreasonably large valid ranges. See Section 8.6 for the interpretation. 


\begin{tabular}{ll|ccccc}
\hline & & SSC & SSC & SSC & AoR & SSC \& AoR \\
& $\sigma_{n}[\mathrm{~Pa}]$ & 1000 & 2000 & 5000 & & \\
\hline$\mu_{s}$ & mean & 0.852 & 0.439 & 0.557 & 0.459 & 0.461 \\
$(-)$ & std. dev. (SD) & 0.106 & 0.057 & 0.102 & 0.149 & 0.029 \\
& range $\left[R_{i}\right]$ & 0.959 & 0.959 & 0.959 & 0.959 & 0.959 \\
& SD / R & 0.111 & 0.059 & 0.106 & 0.155 & 0.031 \\
\hline$\mu_{r}$ & mean & 0.605 & 0.577 & 0.675 & 0.612 & 0.642 \\
$(-)$ & std. dev. (SD) & 0.242 & 0.237 & 0.176 & 0.253 & 0.064 \\
& range $\left[R_{i}\right]$ & 0.950 & 0.950 & 0.950 & 0.950 & 0.950 \\
& SD / R & 0.255 & 0.249 & 0.185 & 0.266 & 0.067 \\
\hline$C o R$ & mean & 0.697 & 0.559 & 0.541 & 0.662 & 0.577 \\
$(-)$ & std. dev. (SD) & 0.041 & 0.068 & 0.036 & 0.123 & 0.031 \\
& range $\left[R_{i}\right]$ & 0.387 & 0.387 & 0.387 & 0.387 & 0.387 \\
& SD / R & 0.107 & 0.176 & 0.092 & 0.317 & 0.079 \\
\hline$\rho_{p}$ & mean & 2823.6 & 2692.3 & 2691.0 & 2755.0 & 2612.3 \\
$(-)$ & std. dev. (SD) & 408.9 & 410.9 & 410.0 & 408.4 & 405.9 \\
& range $\left(R_{i}\right]$ & 1394.6 & 1394.6 & 1394.6 & 1394.6 & 1394.6 \\
& SD / R & 0.3 & 0.3 & 0.3 & 0.3 & 0.3 \\
\hline valid & number & 156816 & 119311 & 119330 & 784045 & 10006 \\
combinations & $(\%)$ & 0.05 & 0.04 & 0.04 & 0.26 & 0.00 \\
\hline
\end{tabular}

Table 8.11: Valid DEM values for iron ore coarse. For each parameter we show the valid parameter statistics in the the tests and in their intersection. Finally, we show the number of valid parameter combinations over the total (3062500).

\begin{tabular}{ll|ccccccc}
\hline & & SSC & SSC & SSC & SSC & SSC & AoR & SSC \& AoR \\
& $\sigma_{n}[\mathrm{~Pa}]$ & 1000 & 2000 & 5000 & 10000 & 15000 & & \\
\hline$\mu_{s}$ & mean & 0.352 & 0.356 & 0.314 & 0.373 & 0.462 & 0.459 & 0.379 \\
$(-)$ & std. dev. (SD) & 0.029 & 0.031 & 0.027 & 0.025 & 0.055 & 0.149 & 0.020 \\
& range $\left(R_{i}\right)$ & 0.959 & 0.959 & 0.959 & 0.959 & 0.959 & 0.959 & 0.959 \\
& SD / R & 0.030 & 0.033 & 0.028 & 0.026 & 0.057 & 0.155 & 0.021 \\
\hline$\mu_{r}$ & mean & 0.192 & 0.218 & 0.264 & 0.527 & 0.481 & 0.612 & 0.313 \\
$(-)$ & std. dev. (SD) & 0.122 & 0.139 & 0.156 & 0.239 & 0.258 & 0.253 & 0.093 \\
& range (Ri) & 0.950 & 0.950 & 0.950 & 0.950 & 0.950 & 0.950 & 0.950 \\
& SD / R & 0.128 & 0.146 & 0.164 & 0.251 & 0.271 & 0.266 & 0.098 \\
\hline$C o R$ & mean & 0.818 & 0.815 & 0.806 & 0.869 & 0.702 & 0.662 & 0.736 \\
$(-)$ & std. dev. (SD) & 0.068 & 0.069 & 0.070 & 0.025 & 0.085 & 0.123 & 0.045 \\
& range $\left(R_{i}\right)$ & 0.387 & 0.387 & 0.387 & 0.387 & 0.387 & 0.387 & 0.387 \\
& SD / R & 0.176 & 0.177 & 0.181 & 0.065 & 0.221 & 0.317 & 0.116 \\
\hline$\rho_{p}$ (-) & mean & 2817.5 & 2820.0 & 2838.5 & 2792.8 & 2765.0 & 2755.0 & 2980.5 \\
& std. dev. (SD) & 403.2 & 399.2 & 394.7 & 419.4 & 411.9 & 408.4 & 396.3 \\
& range $\left(R_{i}\right)$ & 1394.6 & 1394.6 & 1394.6 & 1394.6 & 1394.6 & 1394.6 & 1394.6 \\
\hline valid & SD / R & 0.3 & 0.3 & 0.3 & 0.3 & 0.3 & 0.3 & 0.3 \\
combinations & number & 35334 & 41172 & 57777 & 31413 & 271301 & 784045 & 170 \\
\hline
\end{tabular}

Table 8.12: Valid DEM values for iron ore fine. For each parameter we show the valid parameter statistics in the the tests and in their intersection. Finally, we show the number of valid parameter combinations over the total (3062500). 


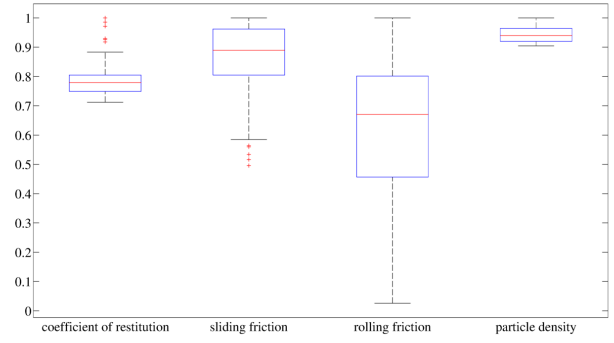

(a) Box plot $\mathrm{SCT}$

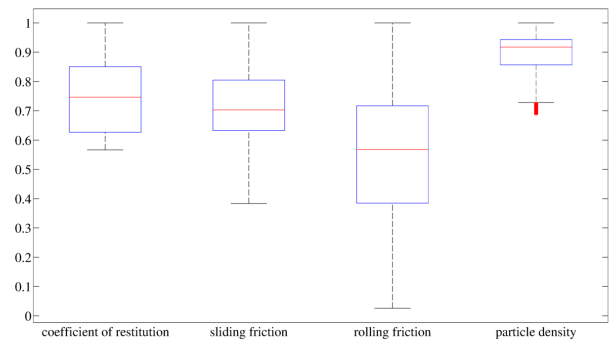

(c) Box plot AoR

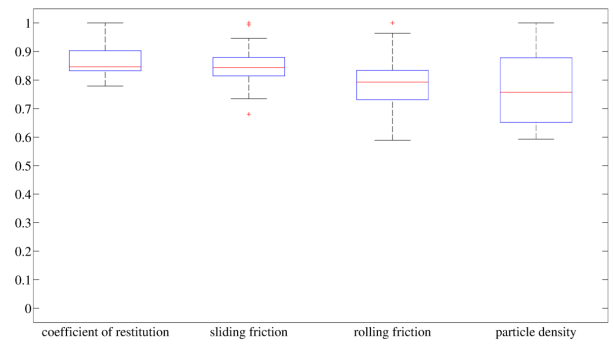

(e) Box plot intersection: AoR \& SCT

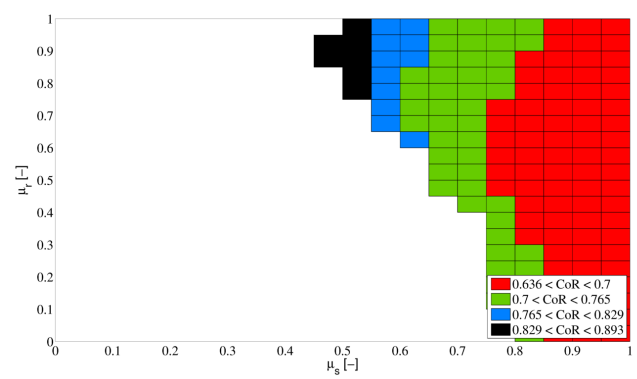

(b) Density plot $\mathrm{SCT}$

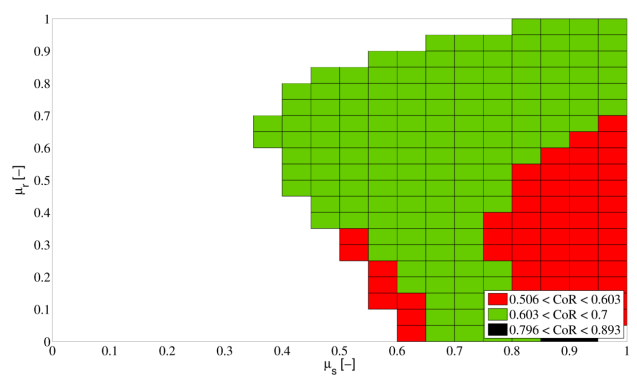

(d) Density plot AoR

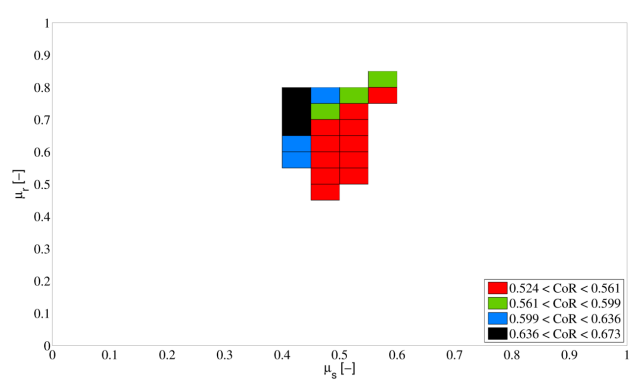

(f) Density plot intersection: AoR \& $\mathrm{SCT}$

Figure 8.14: Iron ore coarse valid values. The valid values for the $\mathrm{SCT}$ and $\mathrm{AoR}$ tests are shown, together with the merge values, valid for both. The plots referring to the single test show reasonably narrow confidence ranges, while Fig. 8.14e shows unreasonably large valid ranges. See Section 8.6 for the interpretation. 


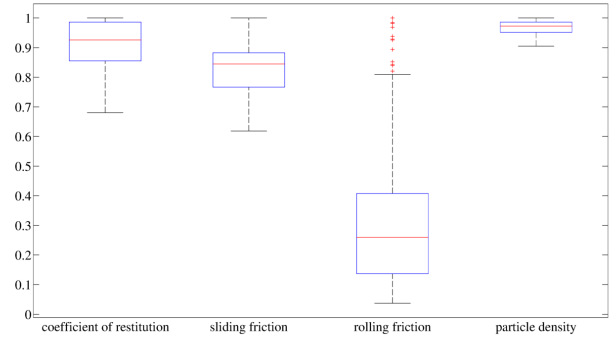

(a) Box plot $\mathrm{SCT}$

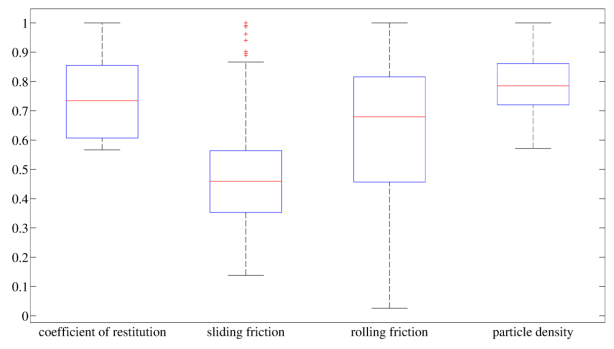

(c) Box plot AoR

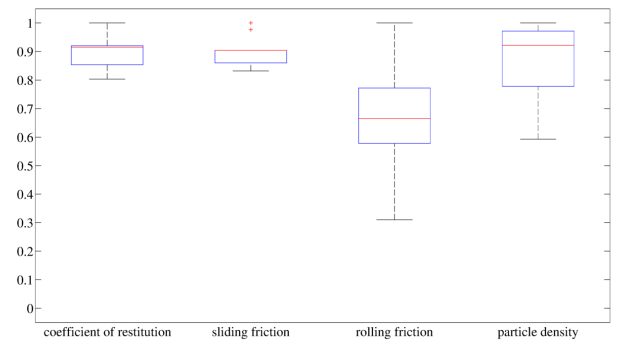

(e) Box plot intersection: AoR \& SCT

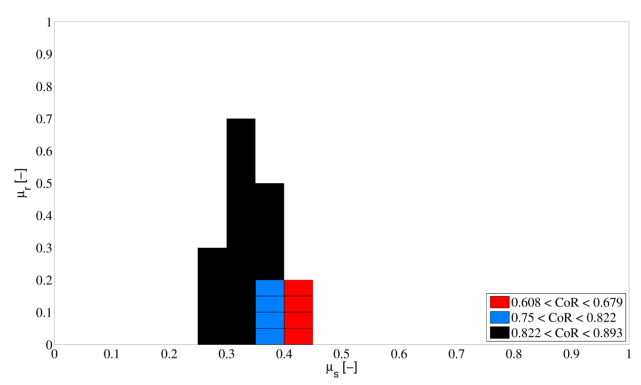

(b) Density plot $\mathrm{SCT}$

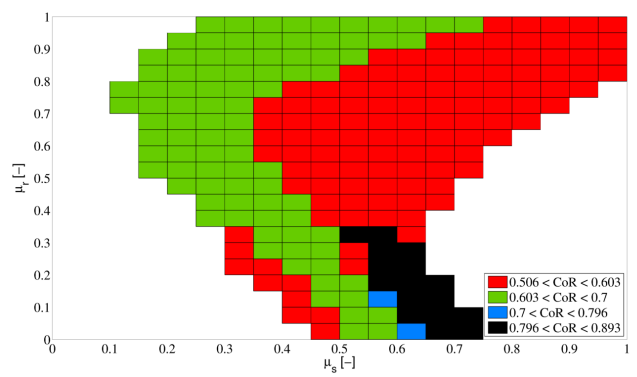

(d) Density plot AoR

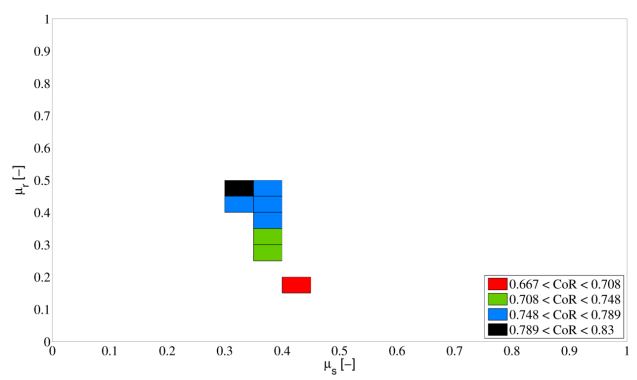

(f) Density plot intersection: AoR \& SCT

Figure 8.15: Iron ore fine valid values. The valid values for the $\mathrm{SCT}$ and $\mathrm{AoR}$ tests are shown, together with the merge values, valid for both. The plots referring to the single test show reasonably narrow confidence ranges, while Fig. $8.15 \mathrm{e}$ shows unreasonably large valid ranges. See Section 8.6 for the interpretation. 


\begin{tabular}{ll|cccc}
\hline & & SSC & SSC & AoR & SSC 2000 \\
& $\sigma_{n}[\mathrm{~Pa}]$ & 1000 & 2000 & & \& AoR \\
\hline$\mu_{s}$ & mean & 0.278 & 0.224 & 0.459 & 0.275 \\
$(-)$ & std. dev. (SD) & 0.015 & 0.005 & 0.149 & 0.011 \\
& range $\left[R_{i}\right]$ & 0.959 & 0.959 & 0.959 & 0.959 \\
& SD / R & 0.016 & 0.005 & 0.155 & 0.012 \\
\hline$\mu_{r}$ & mean & 0.748 & 0.563 & 0.612 & 0.731 \\
$(-)$ & std. dev. (SD) & 0.085 & 0.054 & 0.253 & 0.069 \\
& range $\left[R_{i}\right)$ & 0.950 & 0.950 & 0.950 & 0.950 \\
& SD / R & 0.090 & 0.057 & 0.266 & 0.072 \\
\hline$C o R$ & mean & 0.518 & 0.518 & 0.662 & 0.519 \\
$(-)$ & std. dev. (SD) & 0.011 & 0.010 & 0.123 & 0.011 \\
& range $\left(R_{i}\right)$ & 0.387 & 0.387 & 0.387 & 0.387 \\
& SD / R & 0.028 & 0.026 & 0.317 & 0.028 \\
\hline$\rho_{p}$ & mean & 2201.6 & 2029.4 & 2755.0 & 2197.7 \\
$(-)$ & std. dev. (SD) & 120.1 & 0.0 & 408.4 & 118.6 \\
& range $\left(R_{i}\right)$ & 1394.6 & 1394.6 & 1394.6 & 1394.6 \\
& SD / R & 0.1 & 0.0 & 0.3 & 0.1 \\
\hline valid & number & 1733 & 111 & 784045 & 1561 \\
combinations & $(\%)$ & 0.00 & 0.00 & 0.26 & 0.00 \\
\hline
\end{tabular}

Table 8.13: Valid DEM values for limestone coarse. For each parameter we show the valid parameter statistics in the the tests and in their intersection. Finally, we show the number of valid parameter combinations over the total (3062500).

\begin{tabular}{ll|cccccc}
\hline & & SSC & SSC & SSC & SSC & AoR & SSC \& AoR \\
& $\sigma_{n}[\mathrm{~Pa}]$ & 1000 & 2000 & 5000 & 10000 & & \\
\hline$\mu_{s}$ & mean & 0.300 & 0.213 & 0.288 & 0.331 & 0.708 & 0.308 \\
$(-)$ & std. dev. (SD) & 0.053 & 0.044 & 0.040 & 0.040 & 0.129 & 0.032 \\
& range $\left[R_{i}\right]$ & 0.959 & 0.959 & 0.959 & 0.959 & 0.959 & 0.959 \\
& SD / R & 0.055 & 0.046 & 0.042 & 0.042 & 0.134 & 0.033 \\
\hline$\mu_{r}$ & mean & 0.576 & 0.512 & 0.224 & 0.577 & 0.526 & 0.645 \\
$(-)$ & std. dev. (SD) & 0.222 & 0.191 & 0.114 & 0.257 & 0.220 & 0.106 \\
& range $\left[R_{i}\right)$ & 0.950 & 0.950 & 0.950 & 0.950 & 0.950 & 0.950 \\
& SD / R & 0.234 & 0.202 & 0.120 & 0.271 & 0.231 & 0.112 \\
\hline$C o R$ & mean & 0.649 & 0.703 & 0.698 & 0.778 & 0.667 & 0.634 \\
$(-)$ & std. dev. (SD) & 0.110 & 0.118 & 0.124 & 0.079 & 0.114 & 0.051 \\
& range $\left[R_{i}\right)$ & 0.387 & 0.387 & 0.387 & 0.387 & 0.387 & 0.387 \\
& SD / R & 0.285 & 0.305 & 0.321 & 0.203 & 0.296 & 0.133 \\
\hline$\rho_{p}$ & mean & 2454.6 & 2473.6 & 2453.1 & 2170.5 & 2739.4 & 2404.2 \\
$(-)$ & std. dev. (SD) & 228.0 & 235.4 & 226.4 & 105.9 & 410.1 & 231.2 \\
& range $\left[R_{i}\right]$ & 1394.6 & 1394.6 & 1394.6 & 1394.6 & 1394.6 & 1394.6 \\
& SD / R & 0.2 & 0.2 & 0.2 & 0.1 & 0.3 & 0.2 \\
\hline valid & number & 106966 & 88907 & 53567 & 15514 & 1089496 & 25714 \\
combinations & $(\%)$ & 0.03 & 0.03 & 0.02 & 0.01 & 0.36 & 0.01 \\
\hline
\end{tabular}

Table 8.14: Valid DEM values for limestone fine. For each parameter we show the valid parameter statistics in the the tests and in their intersection. Finally, we show the number of valid parameter combinations over the total (3062500). 


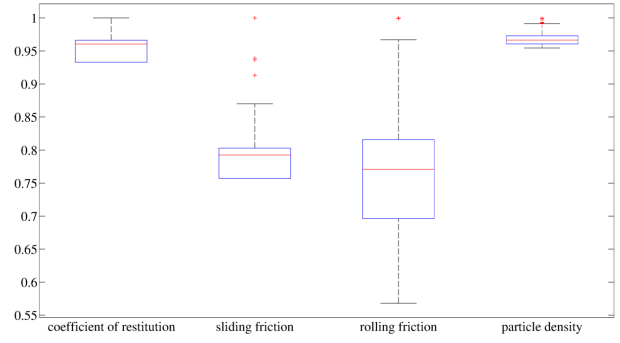

(a) Box plot $\mathrm{SCT}$

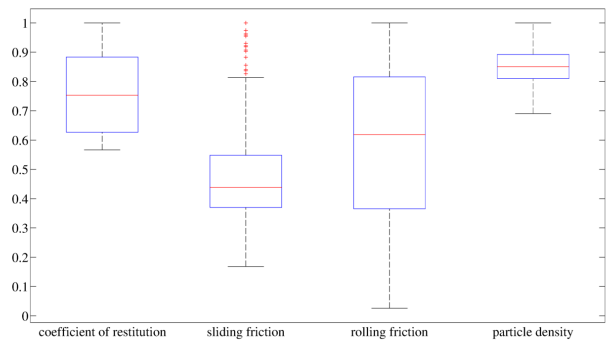

(c) Box plot AoR

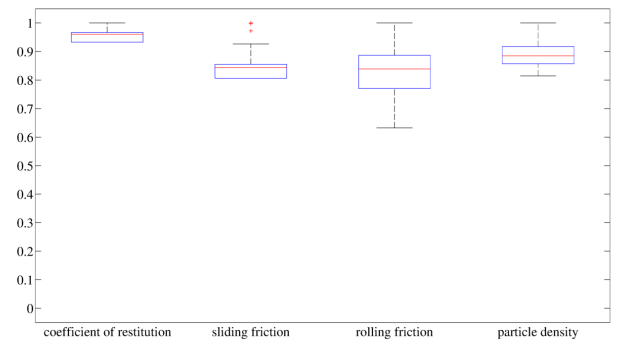

(e) Box plot intersection: AoR \& SCT

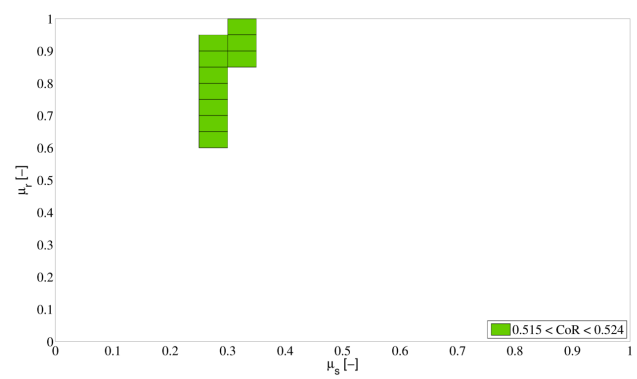

(b) Density plot $\mathrm{SCT}$

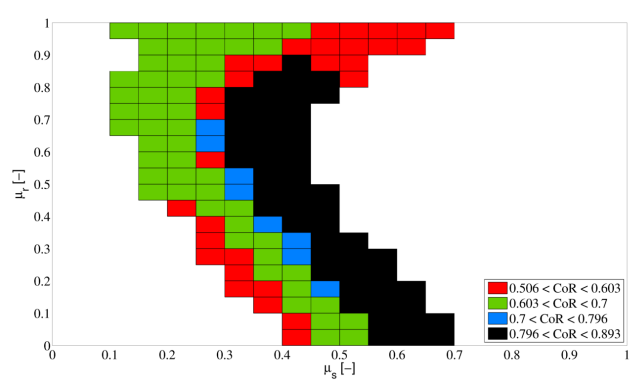

(d) Density plot AoR

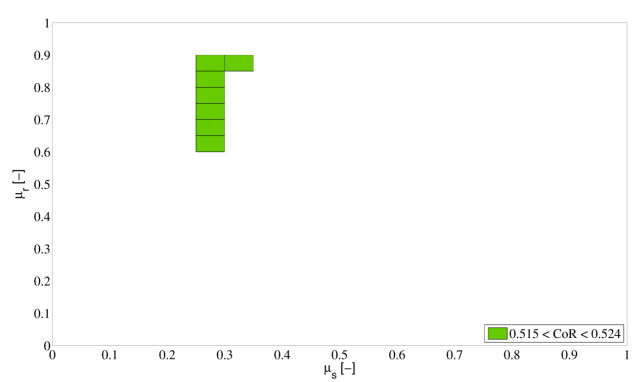

(f) Density plot intersection: AoR \& $\mathrm{SCT}$

Figure 8.16: Limestone coarse valid values. The valid values for the SCT and AoR tests are shown, together with the merge values, valid for both. The plots referring to the single test show reasonably narrow confidence ranges, while Fig. 8.16e shows unreasonably large valid ranges. See Section 8.6 for the interpretation. 


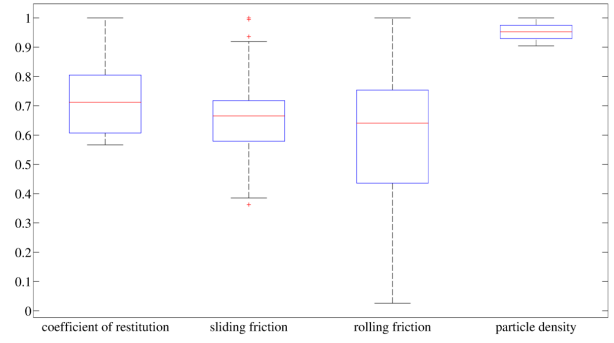

(a) Box plot $\mathrm{SCT}$

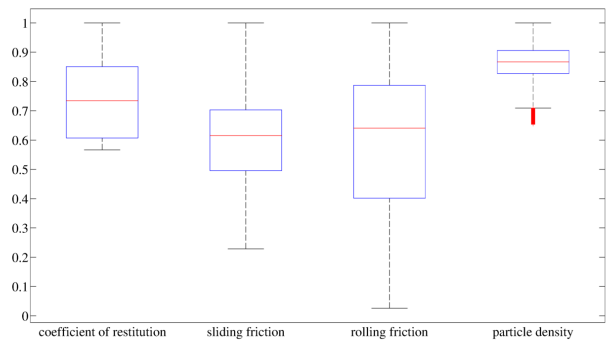

(c) Box plot AoR

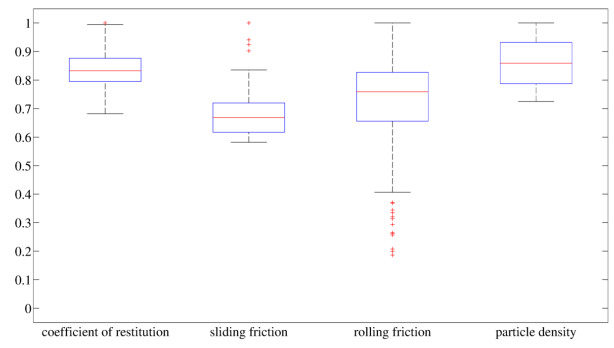

(e) Box plot intersection: AoR \& SCT

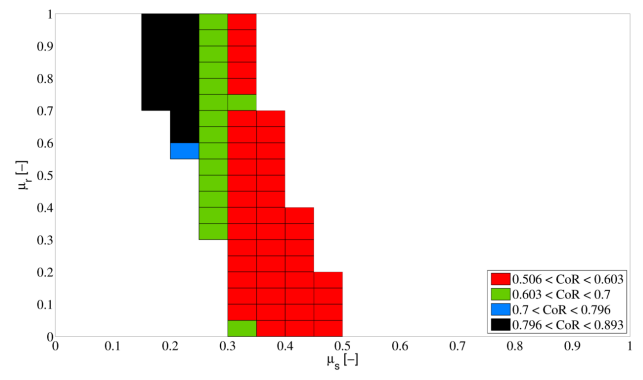

(b) Density plot $\mathrm{SCT}$

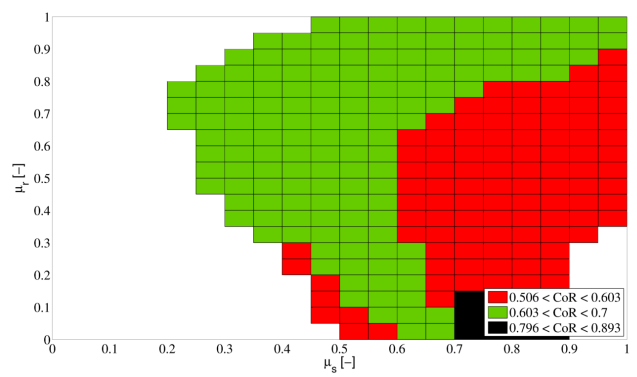

(d) Density plot AoR

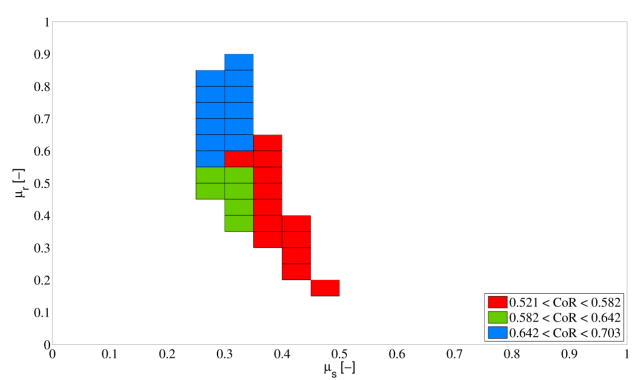

(f) Density plot intersection: AoR \& SCT

Figure 8.17: Limestone fine valid values. The valid values for the $\$ \mathrm{ST}$ and $\mathrm{AoR}$ tests are shown, together with the merge values, valid for both. The plots referring to the single test show reasonably narrow confidence ranges, while Fig. $8.17 \mathrm{e}$ shows unreasonably large valid ranges. See Section 8.6 for the interpretation. 


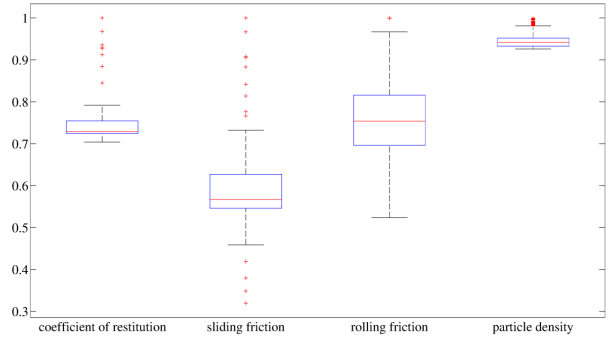

(a) Box plot $\mathrm{SCT}$

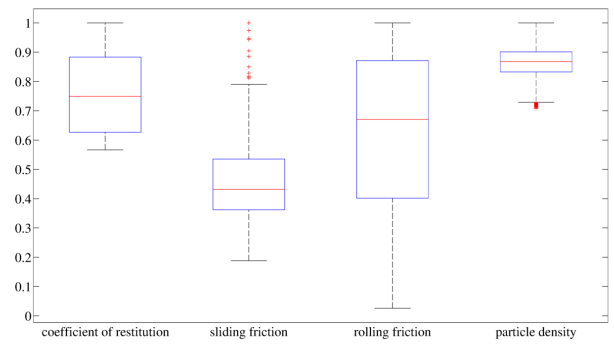

(c) Box plot AoR

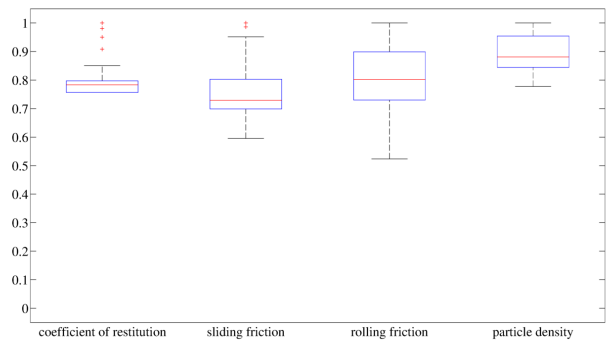

(e) Box plot intersection: AoR \& SCT

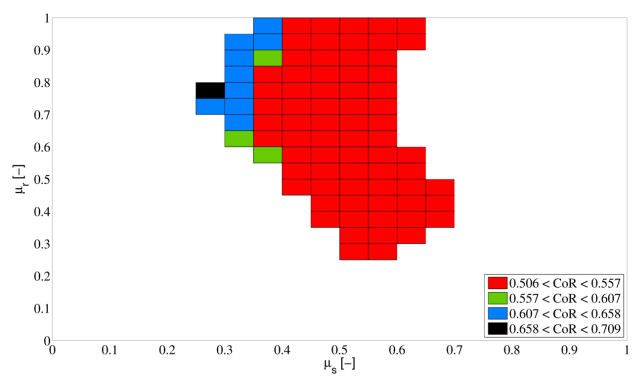

(b) Density plot $\mathrm{SCT}$

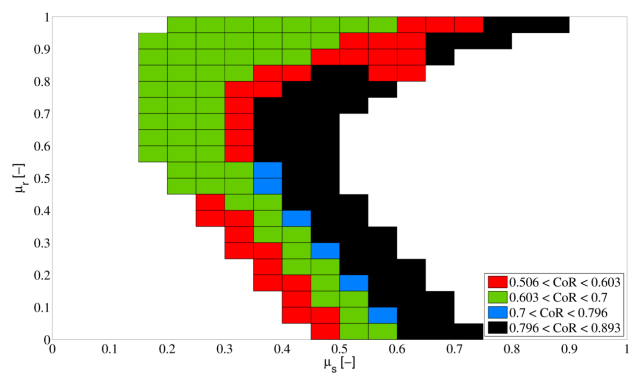

(d) Density plot AoR

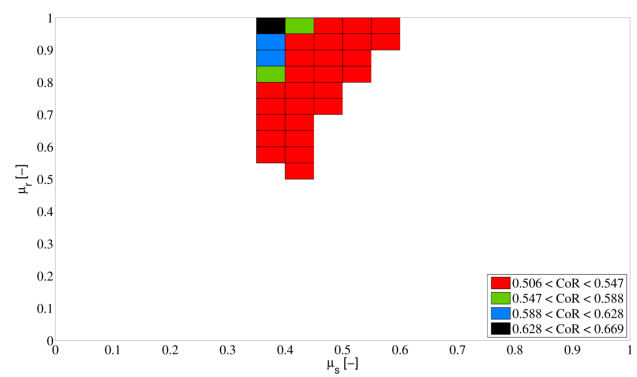

(f) Density plot intersection: AoR \& $\mathrm{SCT}$

Figure 8.18: Sinter coarse valid values. The valid values for the SCT and AoR tests are shown, together with the merge values, valid for both. The plots referring to the single test show reasonably narrow confidence ranges, while Fig. $8.18 \mathrm{e}$ shows unreasonably large valid ranges. See Section 8.6 for the interpretation. 


\begin{tabular}{ll|ccccc}
\hline & & SSC & SSC & SSC & AoR & SSC \& AoR \\
& $\sigma_{n}[\mathrm{~Pa}]$ & 1000 & 2000 & 5000 & & \\
\hline$\mu_{s}$ & mean & 0.211 & 0.463 & 0.427 & 0.708 & 0.433 \\
& std. dev. (SD) & 0.031 & 0.077 & 0.043 & 0.129 & 0.045 \\
& range $\left.R_{i}\right]$ & 0.959 & 0.959 & 0.959 & 0.959 & 0.959 \\
& SD / R & 0.033 & 0.080 & 0.045 & 0.134 & 0.047 \\
\hline$\mu_{r}$ & mean & 0.752 & 0.696 & 0.726 & 0.526 & 0.794 \\
$(-)$ & std. dev. (SD) & 0.096 & 0.164 & 0.131 & 0.220 & 0.109 \\
& range $\left(R_{i}\right)$ & 0.950 & 0.950 & 0.950 & 0.950 & 0.950 \\
& SD / R & 0.101 & 0.172 & 0.138 & 0.231 & 0.115 \\
\hline$C$ CoR & mean & 0.536 & 0.535 & 0.532 & 0.667 & 0.526 \\
$(-)$ & std. dev. (SD) & 0.033 & 0.029 & 0.023 & 0.114 & 0.018 \\
& range $\left[R_{i}\right)$ & 0.387 & 0.387 & 0.387 & 0.387 & 0.387 \\
& SD / R & 0.086 & 0.076 & 0.059 & 0.296 & 0.047 \\
\hline$\rho_{p}$ & mean & 2398.2 & 2409.5 & 2303.6 & 2739.4 & 2327.7 \\
$(-)$ & std. dev. (SD) & 237.7 & 231.7 & 178.0 & 410.1 & 175.4 \\
& range $\left[R_{i}\right)$ & 1394.6 & 1394.6 & 1394.6 & 1394.6 & 1394.6 \\
& SD / R & 0.2 & 0.2 & 0.1 & 0.3 & 0.1 \\
\hline valid & number & 9413 & 54607 & 20063 & 1089496 & 9332 \\
combinations & $(\%)$ & 0.00 & 0.02 & 0.01 & 0.36 & 0.00 \\
\hline
\end{tabular}

Table 8.15: Valid DEM values for sinter coarse. For each parameter we show the valid parameter statistics in the the tests and in their intersection. Finally, we show the number of valid parameter combinations over the total (3062500).

\subsection{Discussion of Model Limitations}

In our approach we established a relationship between particle scale contact law parameters and macroscopic bulk behaviour by means of ANN In case the chosen contact law is physically correct by its functional dependencies, this micro to macro relationship can be used in a reversed way to identify valid sets of contact law parameters.

However, in case the chosen contact law is not applicable to the granular material under consideration, our procedure might lead to a wrong set of parameters, which might lead to the correct bulk behaviour. In this case a first error (incorrect contact law) might be annulled by another one (wrong parameters). To avoid such misleading results, the functional dependency of the particle based contact law should be chosen with care, taking into account the contact physics in the granular flow regime under investigation. Further, our ANN based parameter identification should be applied to different macroscopic bulk behaviours. If those parameter identifications do not lead to a common set of valid parameters, most probably the chosen contact law was not applicable. 


\section{Part IV}

\section{Applications}




\section{Chapter 9}

\section{Sinter Segregator}

\subsection{Material properties for sinter chute simulation}

The method previously explained allowed to collect data regarding the contact law. We took the average value for each of the DEM parameter from Table 8.6 and we used them in an industrial scale DEM] simulation, with the same software, of an iron ore sintering process, see Fig. 9.1. Especially, we examined the behaviour of these particles in a sinter chute cooler.

\subsection{Sinter chute design}

The particles moved from the top of a specifically designed chute and were collected by moving boxes at the bottom. These boxes were holed at the base, allowing cool air to lower the temperature of the sinter. It was critical to ascertain if the larger particles segregated, by moving to the bottom of the boxes (see Fig. 9.2), while the smaller to the top, allowing a more effective distribution of the cool air. Especially, the regions highlighted in Fig. 9.3 and Fig. 9.4a for the simulation were investigated.

\subsection{Numerical considerations for sinter chute simulation}

The simulation was performed with a maximum of 500,000 particles. For a DEM simulation is a huge number, obtained with a coarse graining factor of five, meaning that all the particles have radii five time larger than reality. Avoiding coarse graining would have meant more than 10,000,000 particles, infeasible for us.

\subsection{Sinter chute simulation phases}

\begin{tabular}{c|cccc}
\hline$\#$ & diameter $[\mathrm{mm}]$ & radius $[\mathrm{m}]$ & coarse grained radius $[\mathrm{m}]$ & $\%$ of the volume \\
\hline 1 & 5 & 0.0025 & 0.0125 & 30 \\
2 & 10 & 0.0050 & 0.0250 & 25 \\
3 & 20 & 0.0100 & 0.0500 & 25 \\
4 & 50 & 0.0250 & 0.1250 & 20 \\
\hline
\end{tabular}

Table 9.1: Particles distribution at the inlet.

Particles are inserted continuously from the top according to the particle distribution in Table 9.1 in two main phases: 


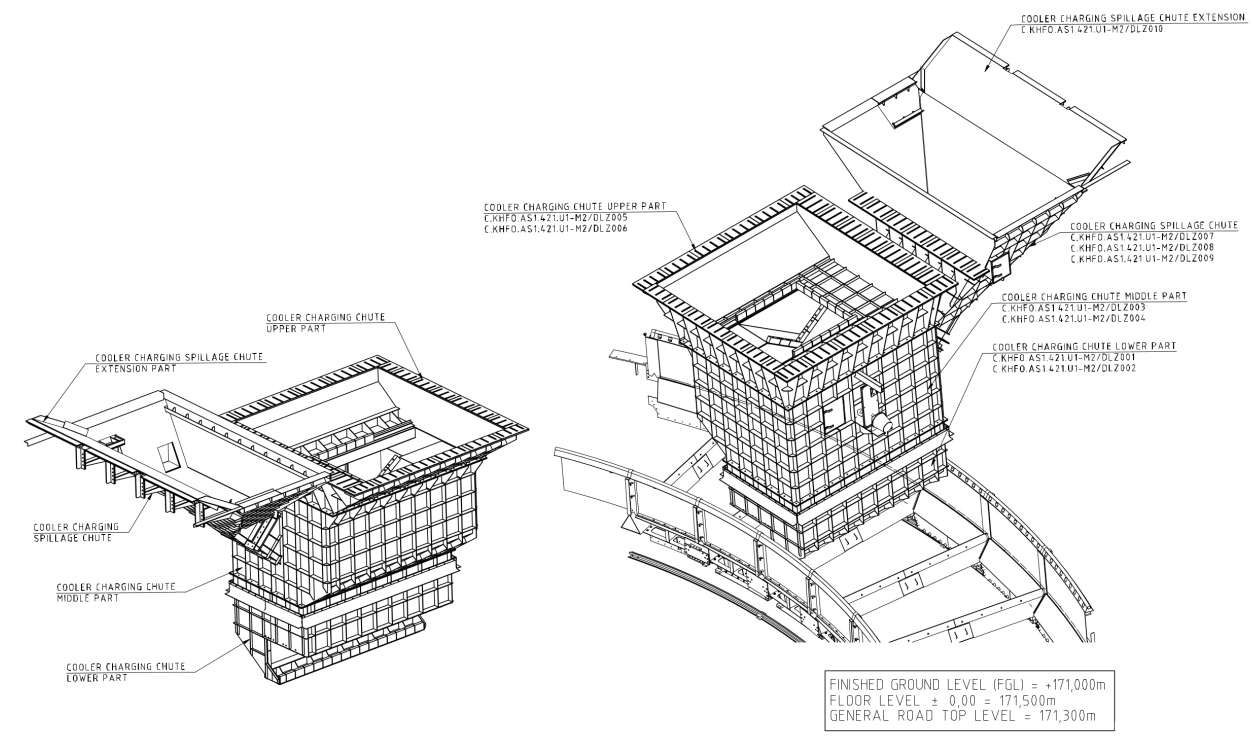

Figure 9.1: Sinter chute orto layout (Primetals GmbH).

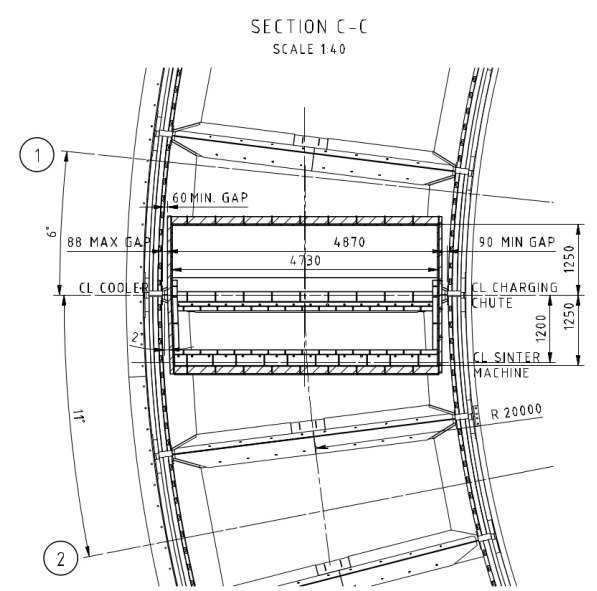

Figure 9.2: Sinter chute box (Primetals GmbH).

\subsubsection{Initial filling}

During the initial phase many of the small particles deposited inside the chute, while the large majority of the large ones reached the bottom box.

\subsubsection{Moving bottom box}

Similarly, also in this phase particles were continuously inserted from the top. After the filling was completed, the scraper was moved and all the particles beyond a defined surface were deleted. Inside the sinter, all the places that small particles could occupy already were. At this point the bottom box was filled again, but with a more realistic size distribution, and it reached steady state. Fig. 9.6 show the progressive variation of the position of the particles inside the numerical setup. 


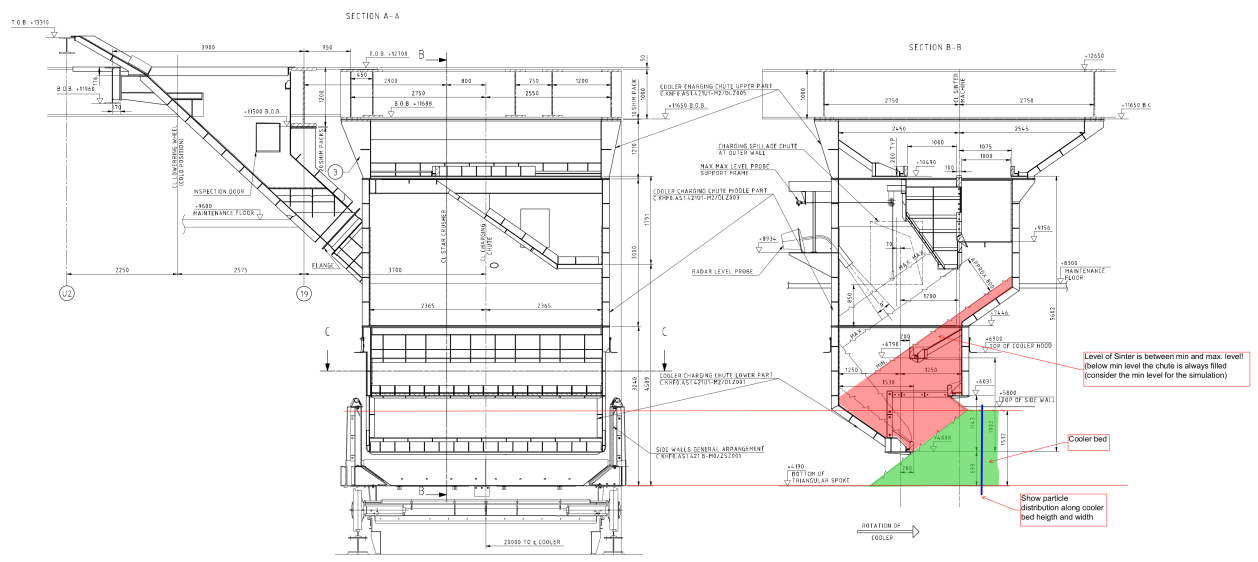

Figure 9.3: Sinter chute vertical layout (Primetals GmbH).

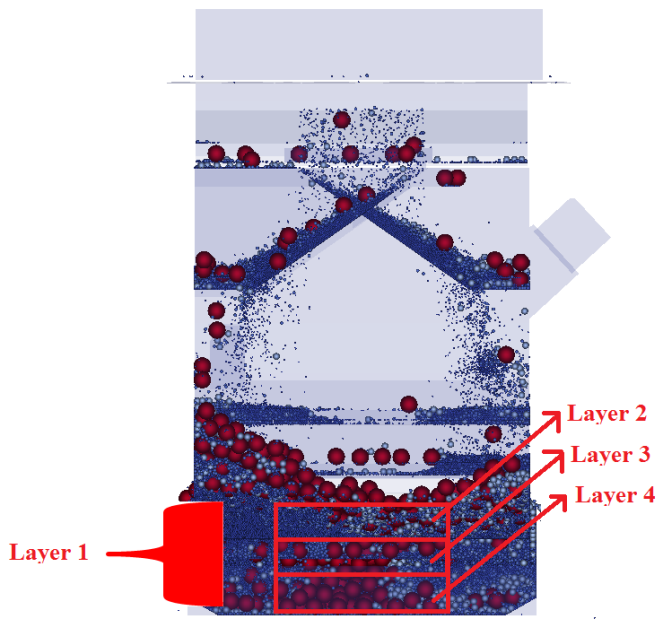

(a) Side view.

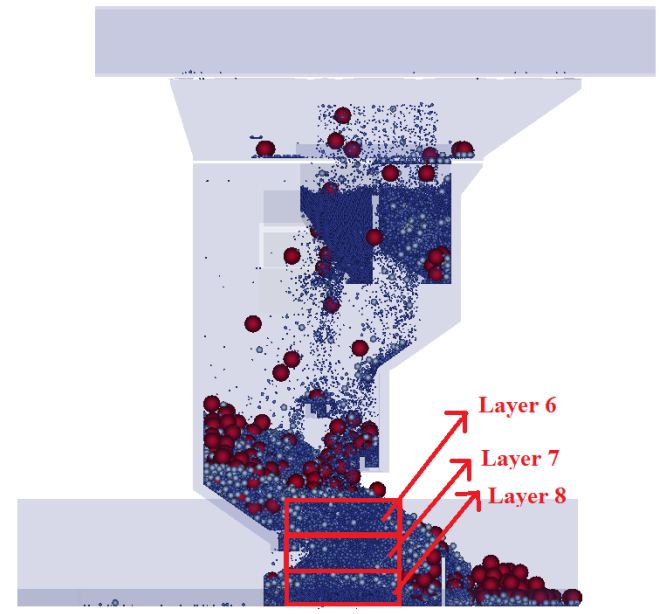

(b) Frontal view.

Figure 9.4: Sinter chute simulation layers. Data for segregation estimate are extracted in these volumes.

\subsection{Sinter chute results}

As expected, when the steady state was reached, the particles actually segregated, as it was intended to demonstrate. We divided the first of the boxes filled by the chute in three (equally high) layers, from 6 on top to 8 on bottom, see Fig. 9.2. The volume over these layers was not considered, because it was continuously supplied of particles from the chute. In fact, there were more particles with larger radii, from 25 to $125 \mathrm{~mm}$, in the bottom layers. Instead, the smallest particles, radius $=12.5 \mathrm{~mm}$, were rather on the top layer. In Fig. 9.5 the percentage of the total volume of the particles available in each layer at steady-state, grouped by radius, is shown. We could clearly see how the larger particles disposed mostly in the bottom layer, validating the realized design.

The DEM parameters used in the simulation can be found in Table 8.6. The total volume of the particles with different radii in the three considered layers, variation with time, can be seen in Fig. 9.7 and 9.8 


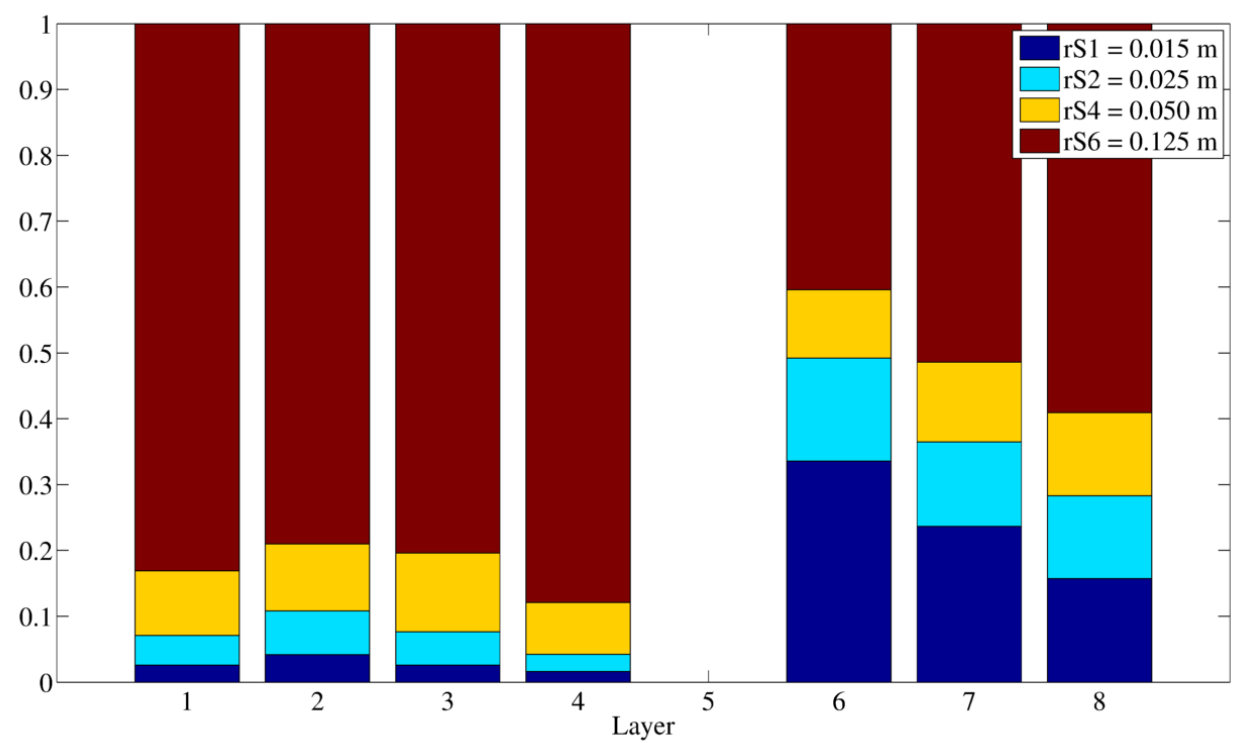

(a) Ratio over the volume occupied by the particles of each radius.

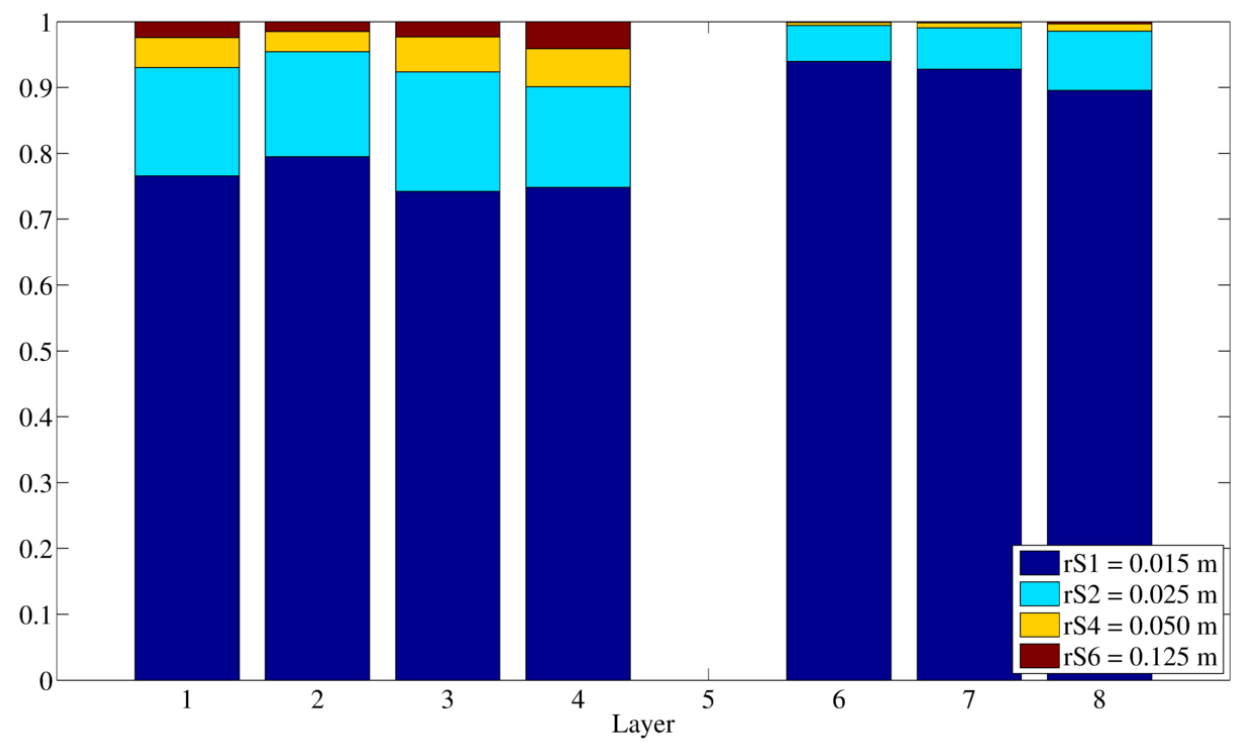

(b) Ratio over the number of the particles of each radius.

Figure 9.5: Sinter bar plot. In the two plots we observe the higher likelihood of finding large particles in the bottom layers and small particles in the top layers. 


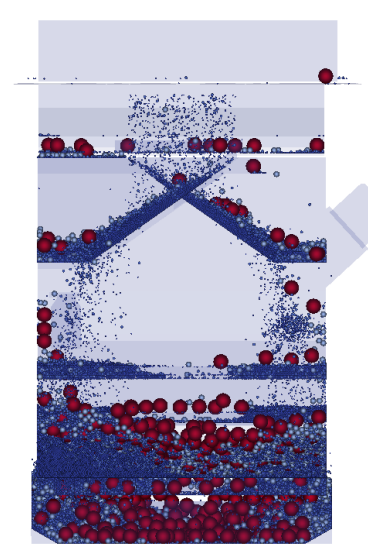

(a) Frontal view. $\mathrm{t}=150 \mathrm{~s}$.

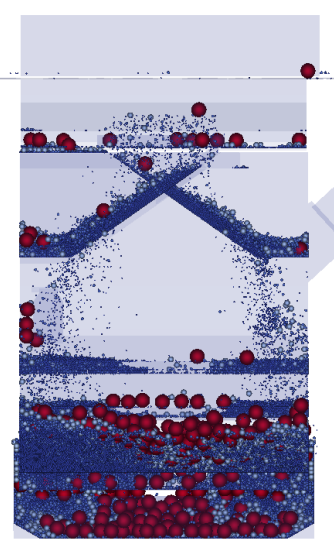

(c) Frontal view. $\mathrm{t}=200 \mathrm{~s}$.

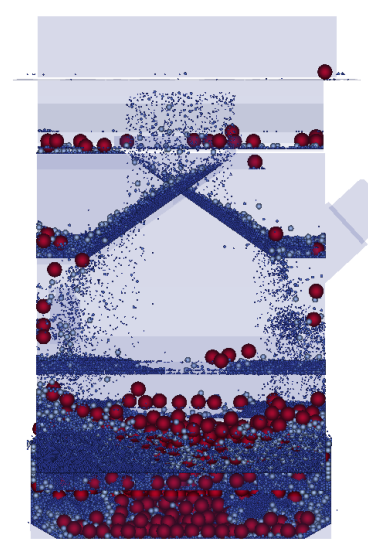

(e) Frontal view. $\mathrm{t}=250 \mathrm{~s}$.

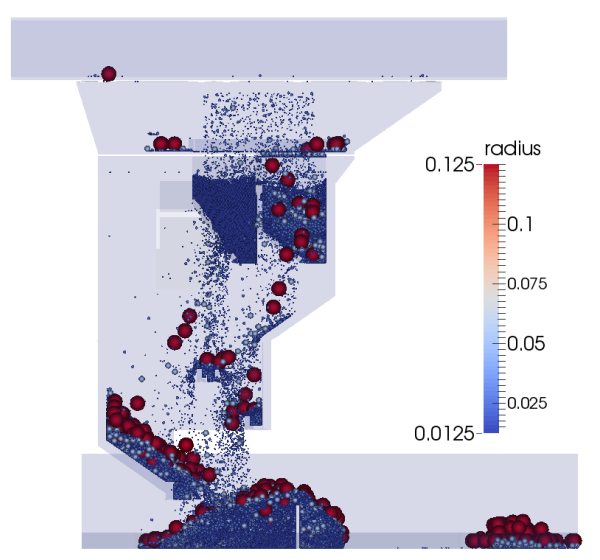

(b) Lateral view. $\mathrm{t}=150 \mathrm{~s}$.

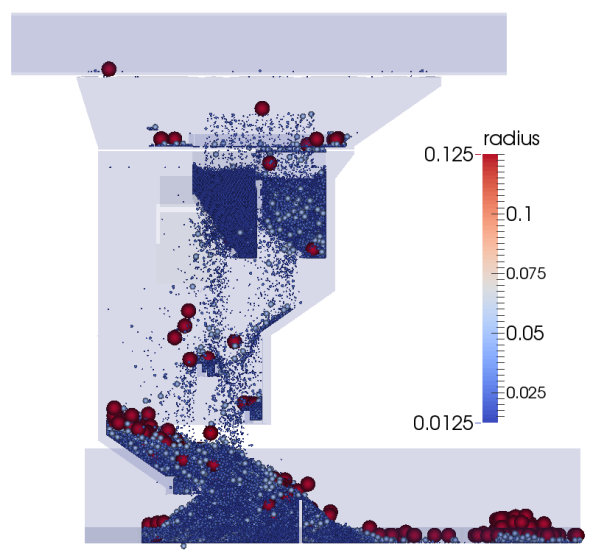

(d) Lateral view. $\mathrm{t}=200 \mathrm{~s}$.

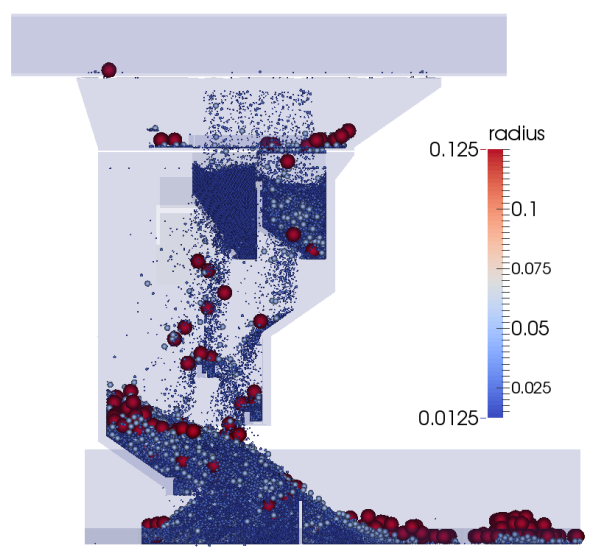

(f) Lateral view. $\mathrm{t}=250 \mathrm{~s}$.

Figure 9.6: Sinter chute simulation screenshots. Particles are tracked to ascertain the segregation behaviour. 

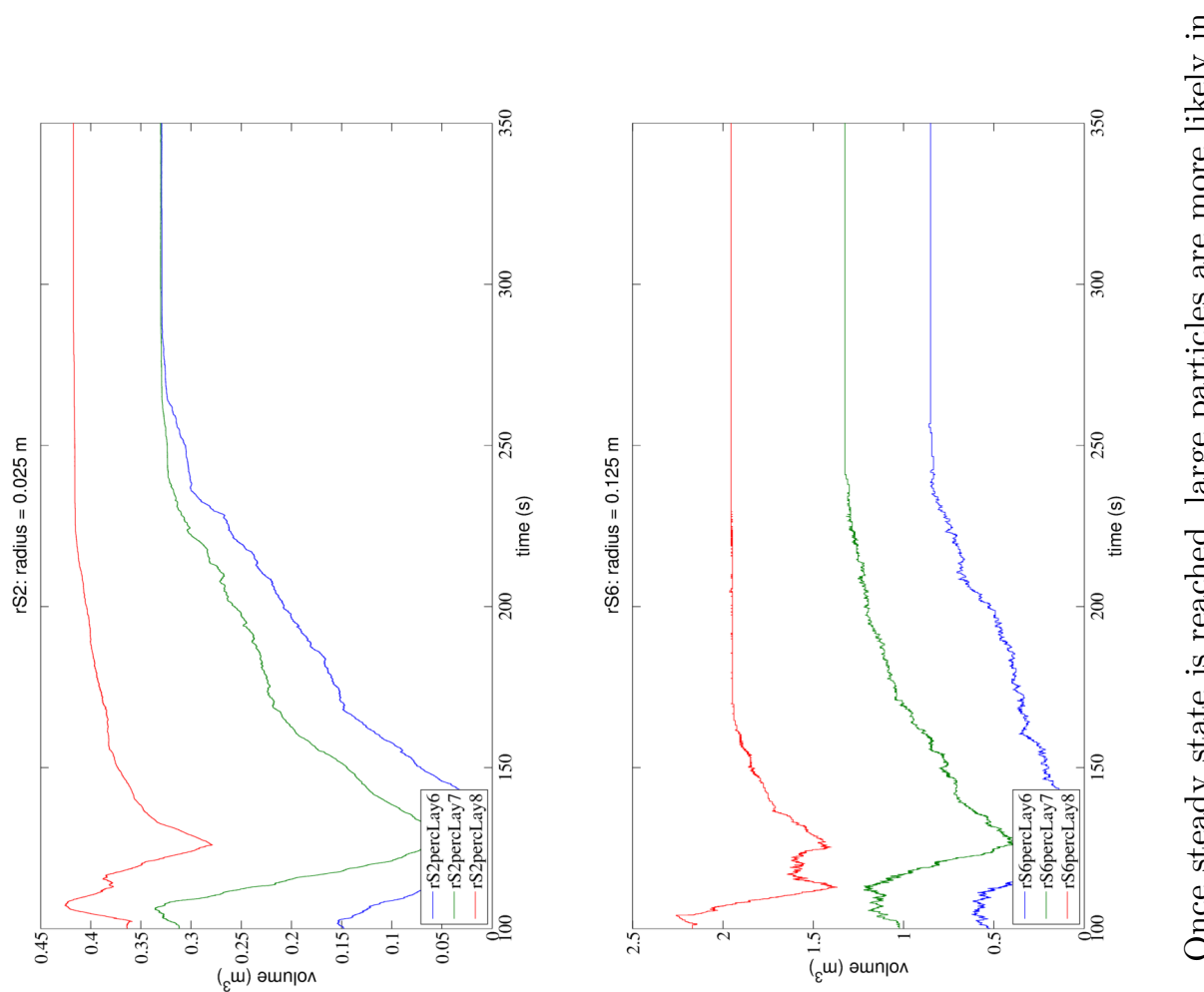

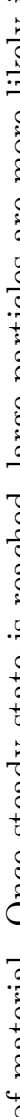
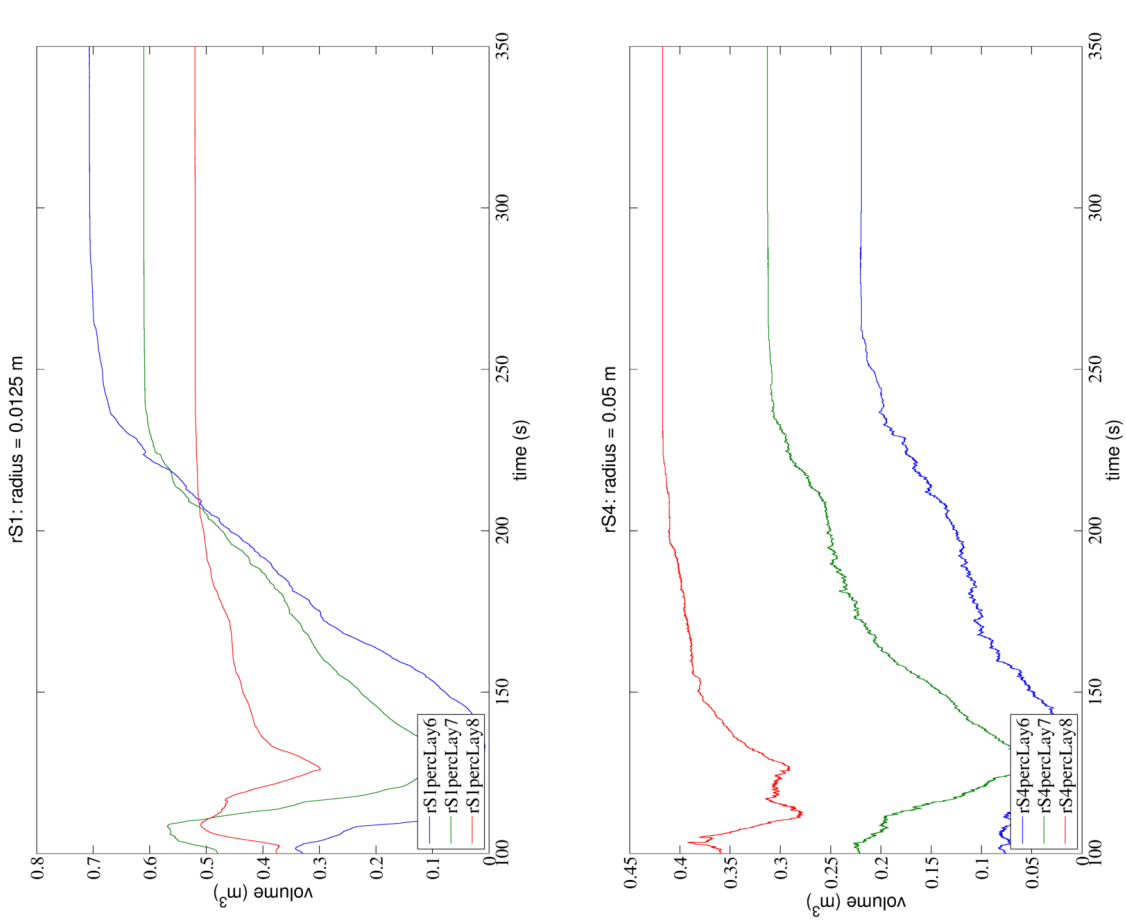

$\varepsilon$

.

$\dot{\mathscr{g}}$

o

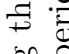

.0

छ

‡.

承

?.

苛

.

$\stackrel{0}{0}$ की

葛

$\ddot{\leftarrow} . \bar{\sigma}$

क

$\stackrel{\Xi}{\exists}$

点 

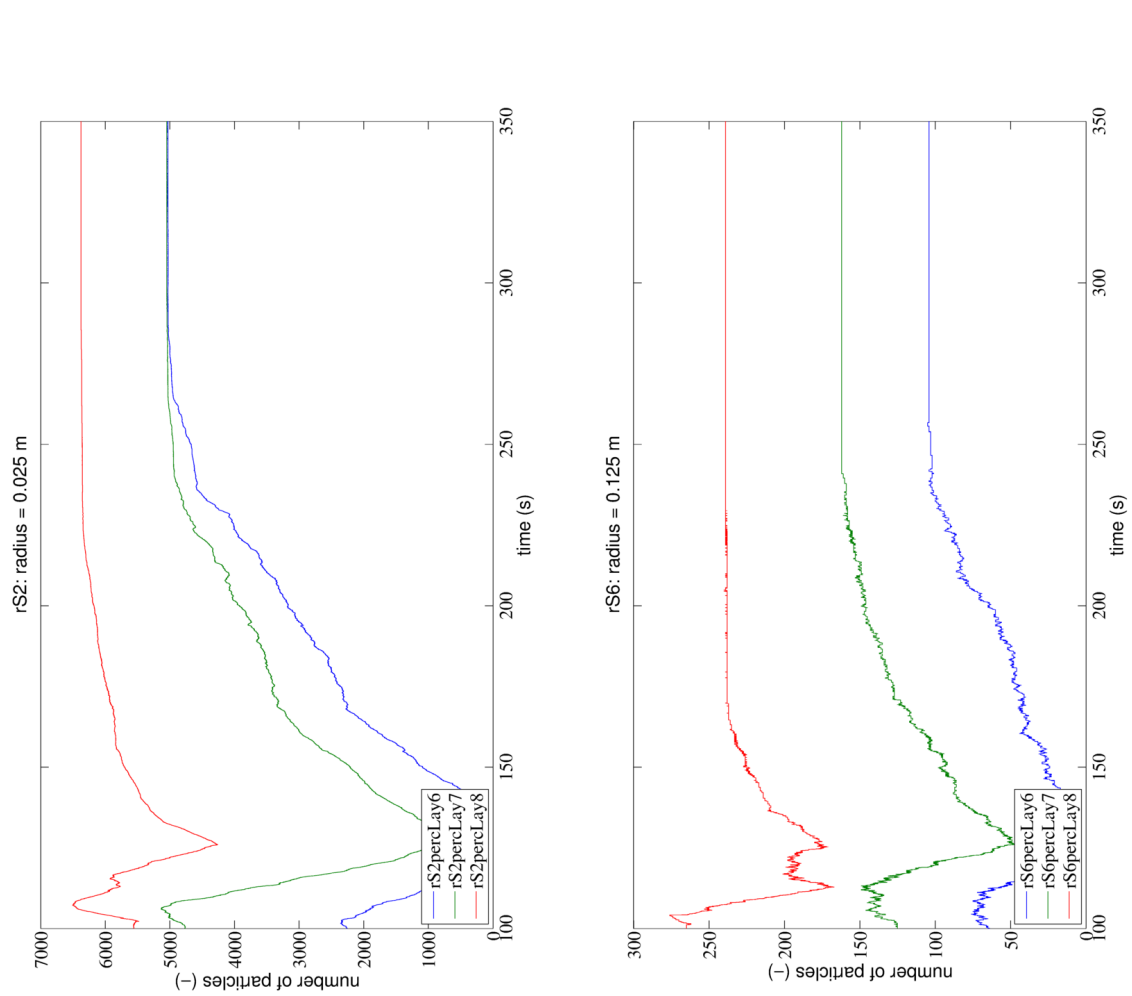

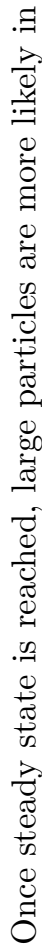
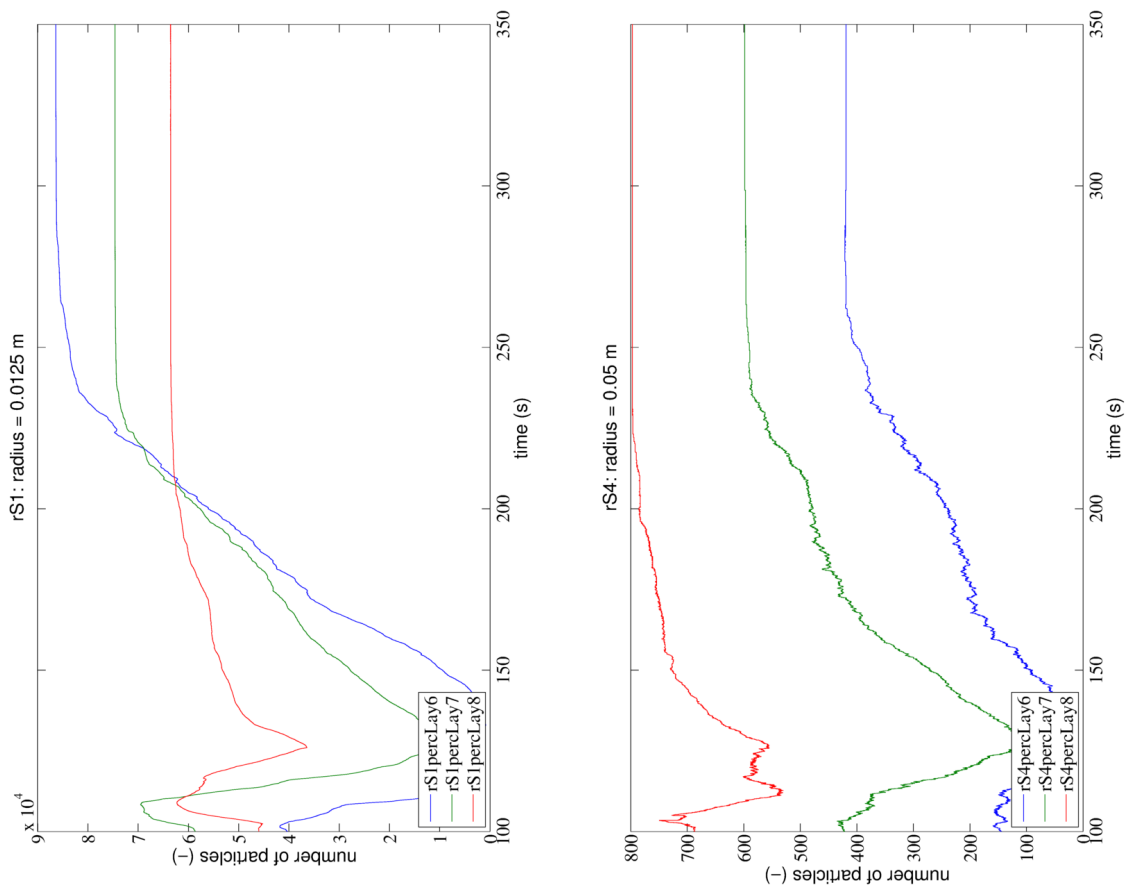

政

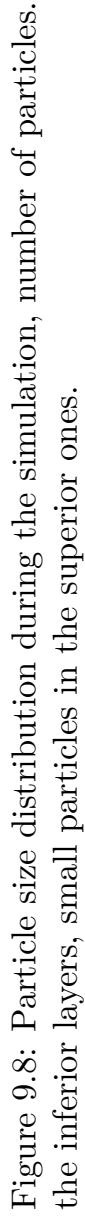




\section{Chapter 10}

\section{Blast Furnace}

\subsection{Blast furnace design}

Well explained in the vast literature ([26]), iron making consists in separating the iron from its chemical combination with oxygen. As of now, the blast furnace (Fig. 10.1a) is considered the most efficient process.

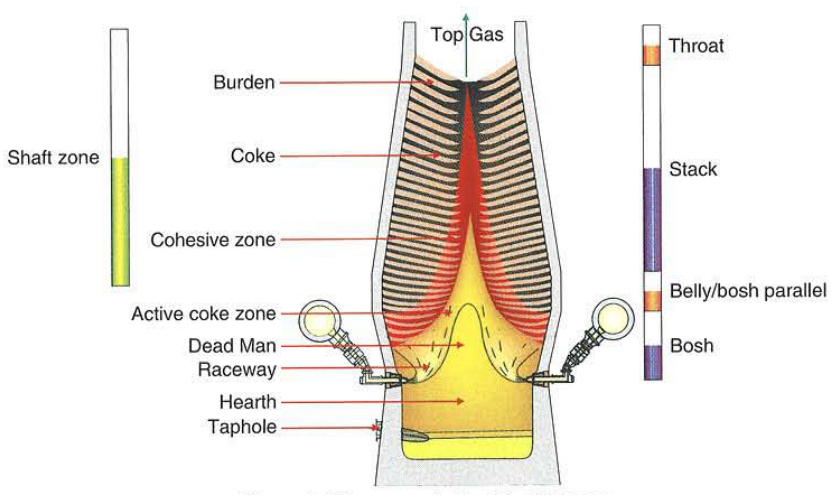

(a) Blast furnace scheme 48 .

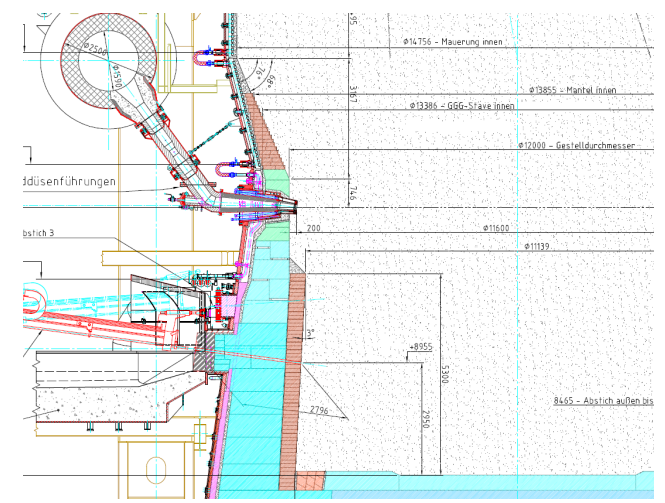

(b) Blast furnace section layout (Voestalpine Stahl $\mathrm{GmbH}$ ).

Figure 10.1: Schematics of the blast furnace investigation.

\subsubsection{Structure}

The blast furnace is a massive vertical apparatus, built with consistent amounts of refractory materials and a robust steel shell. The areas in front of each tuyere, which can be seen in Fig. 10.1a, is called raceway and is the most active. We dedicated this chapter to the investigation of its behaviour.

\subsubsection{Simulation prerequisites}

Given its axial-symmetric geometry and according to Hager 62, we considered only a wedge and applied periodic boundary conditions. Three tuyeres, instead of a single one, and a sufficiently high space over and under them were used to grant uniform conditions. As in Hager [62], 15,000 cells were created for the mesh, shown in Fig. 10.2 The tuyeres were considered as inlets. Air is inserted with a velocity of $100 \mathrm{~m} / \mathrm{s}$, a density of $1.1885 \mathrm{~kg} / \mathrm{m}^{3}$ and a kinematic viscosity of $1.5 \cdot 10^{-5} \mathrm{~m}^{2} / \mathrm{s}$. 
We performed two of simulations of this volume to investigate the effect of the variation of the coefficient of sliding friction $(-)\left(\overline{\mu_{s}}\right)$ :

1. $\mu_{s}=0.1$, or reduced,

2. $\mu_{s}=0.9$, or real, obtained from the parameter identification.

The remaining DEM parameters can be found in Table 10.1 derived from the parameters identified in the previous Chapter 8. The procedure to obtain the equivalent DEM parameters from those of the materials involved can be found in Hager [62, together with the particle distribution, which drove us into using approximately 160,000 particles for each simulation. This amount allowed us to completely filled the setup. On top, a servo-wall, similar to the SCT simulation, consented to apply the correct load conditions inside the system. Each simulation lasted 195 hours on a 32 cores machine.

\begin{tabular}{cccc}
\hline $\begin{array}{c}\text { Young's } \\
\text { modulus }\end{array}$ & $\begin{array}{c}\text { Poisson's } \\
\text { ratio }\end{array}$ & $C_{O} R$ & $\mu_{r}$ \\
{$[M P a]$} & {$[-]$} & {$[-]$} & {$[-]$} \\
\hline 10 & 0.40 & 0.5 & 0.4 \\
\hline
\end{tabular}

Table 10.1: DEM fixed input values for the blast furnace simulation.

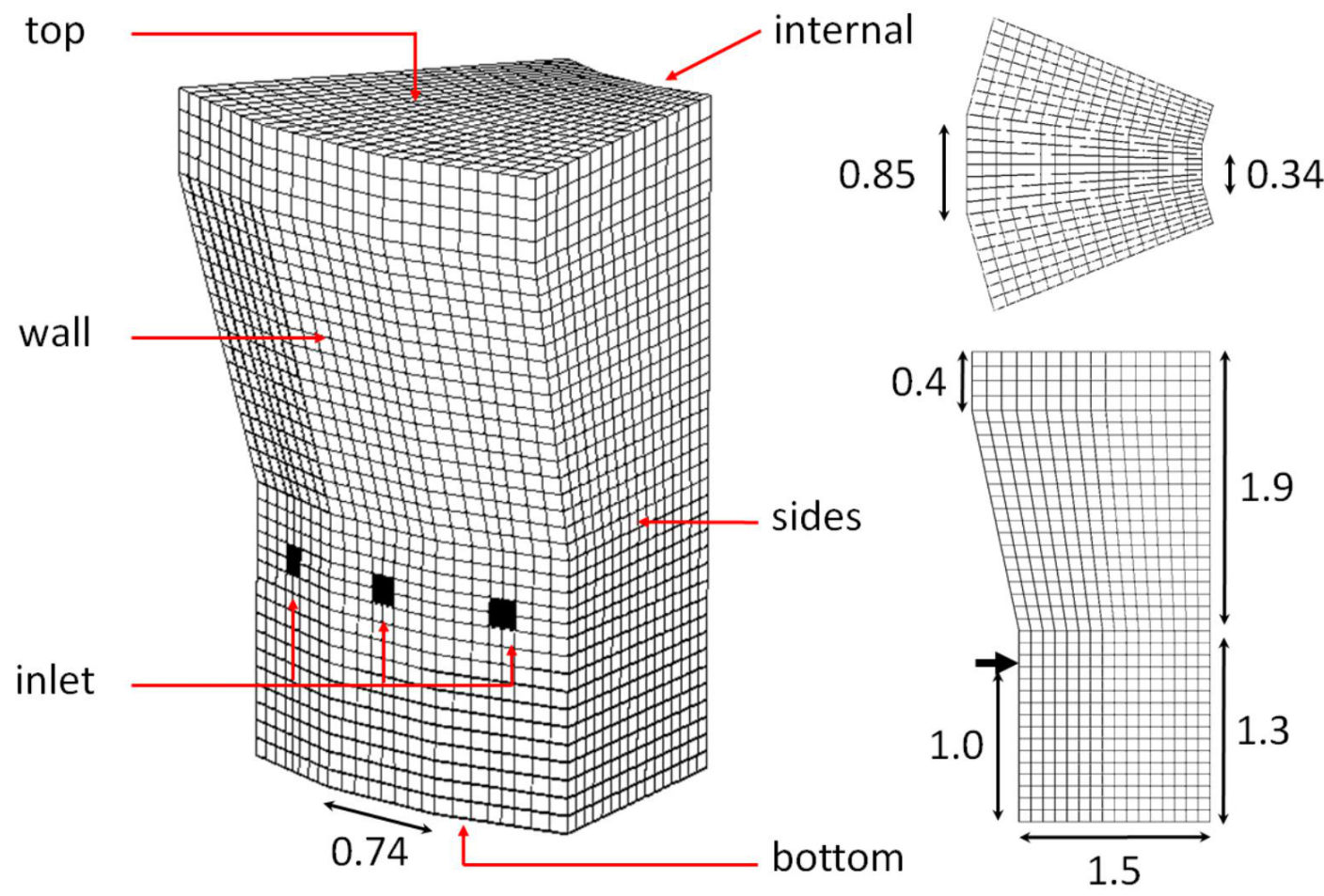

Figure 10.2: Blast furnace simulation layout [62. The dimensions of the domain and the mesh are shown.

\subsection{Results}

In accordance to the literature $([26,62])$ we focused on the gases, or fluid, velocity and the particles velocity in a vertical slice of the simulation domain of the two simulations, in correspondance of the most 
inner inlet. Further, we investigated the penetration depth of the raceway in an horizontal slice of the system, following the indications of Hager [62].

\subsubsection{Fluid velocity}

As thoroughly explained in the literature ([100, 105]), the change of sliding friction coefficients bear limited effect on the velocity of the gases. In Fig. 10.3 we plotted the spatially interpolated values for the fluid velocity, together with the velocity vector arrows and the stream lines, for the two simulations investigated.

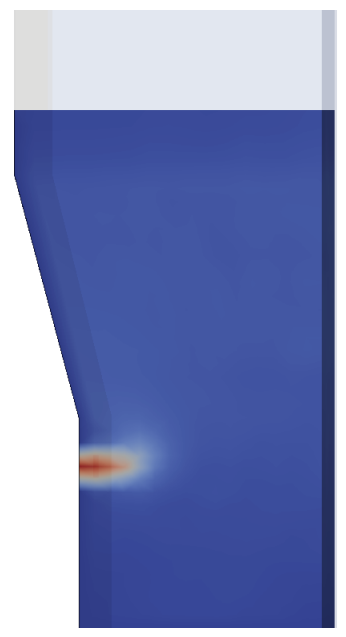

(a) $\mu_{s}=0.1$, spatial average.

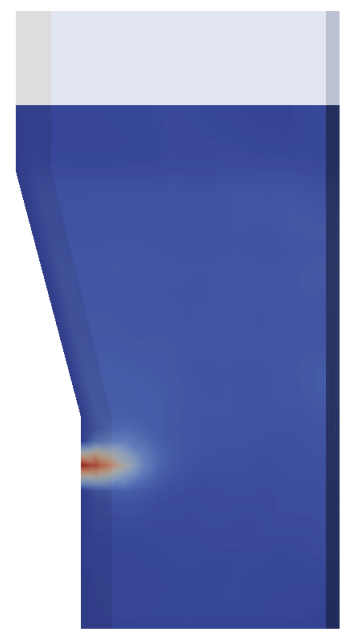

(e) $\mu_{s}=0.9$, spatial average.

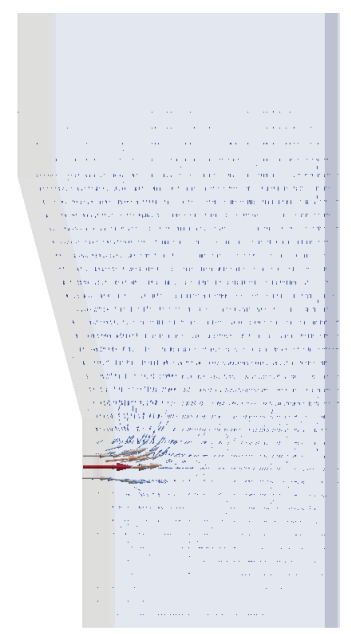

(b) $\mu_{s}=0.1$, velocity vectors.

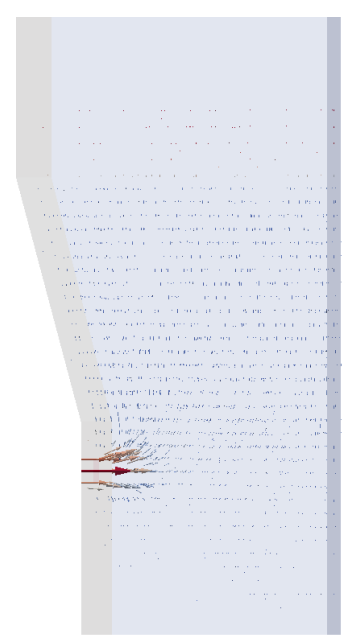

(f) $\mu_{s}=0.9$, velocity vectors.

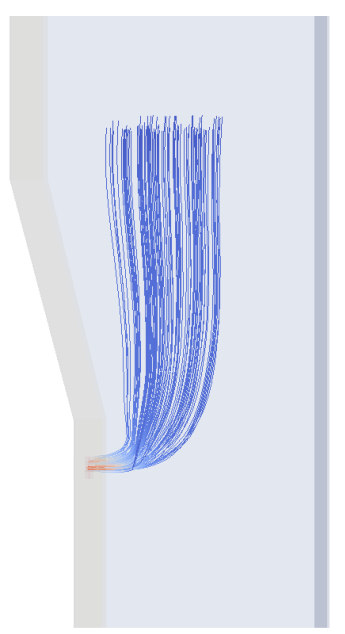

(c) $\mu_{s}=0.1$, stream lines.

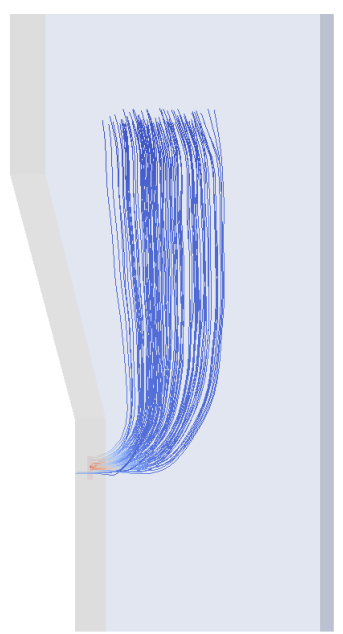

(g) $\mu_{s}=0.9$, stream lines.

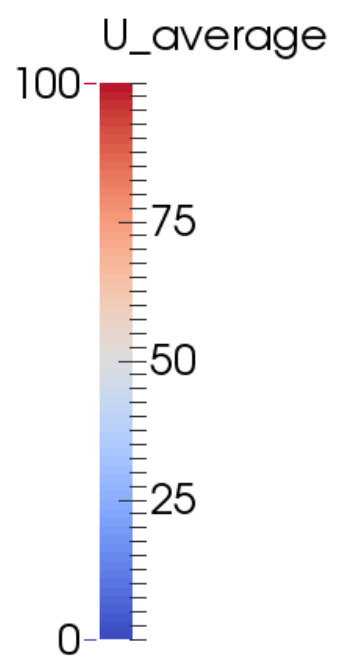

(d) Legend.

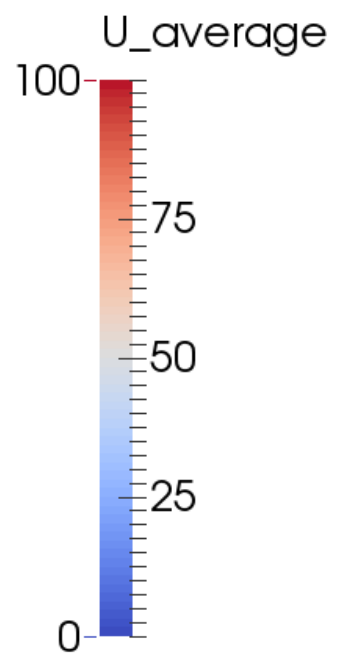

(h) Legend.

Figure 10.3: Vertical slice of fluid velocity. The effect of sliding friction over the fluid velocity is limited. 


\subsubsection{Particle velocity}

On the other hand, and again according to the literature ([100, 105]), the particles velocity is clearly, and logically, higher for low friction particles. They could be moved by the flow more easily compared to high friction particles, see Fig. 10.4. Again, the spatially interpolated values for the particles velocity, together with the velocity vector arrows and the stream lines, for the two simulations investigated, can be seen. In this plot the particles are represented as a fluid occupying the particles space. Thus, a certain degree of smoothing must be taken into account. However, the raceway area is clearly larger in the reduced $\mu_{s}$ simulation, testifying the consistent effect of this parameter in the overall results.

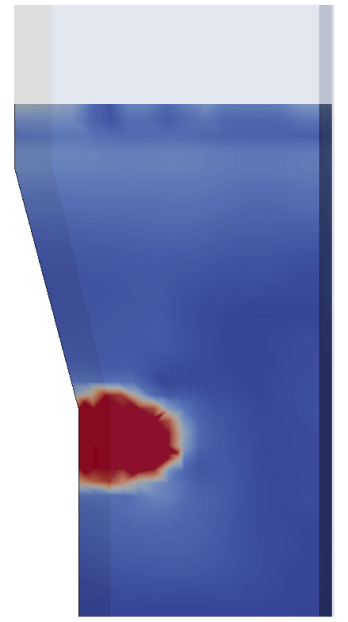

(a) $\mu_{s}=0.1$, spatial average.

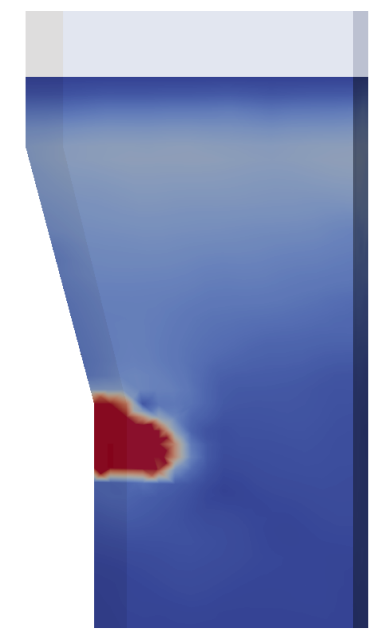

(e) $\mu_{s}=0.9$, spatial average.

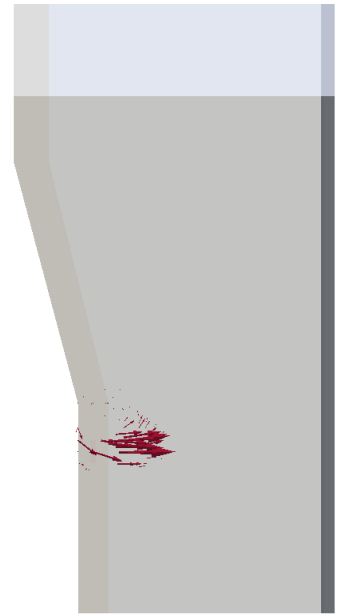

(b) $\mu_{s}=0.1$, velocity vectors.

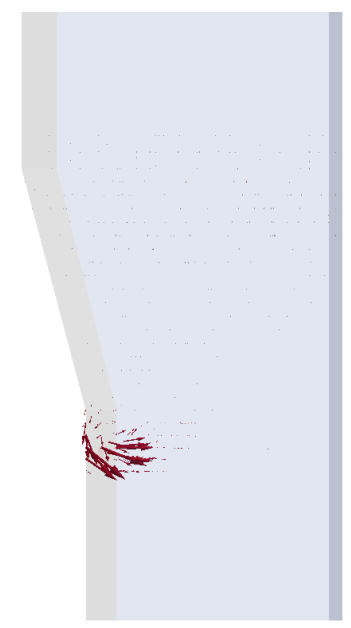

(f) $\mu_{s}=0.9$, velocity vectors.

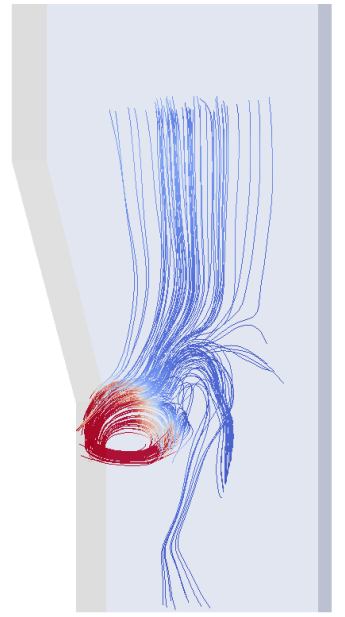

(c) $\mu_{s}=0.1$, stream lines.

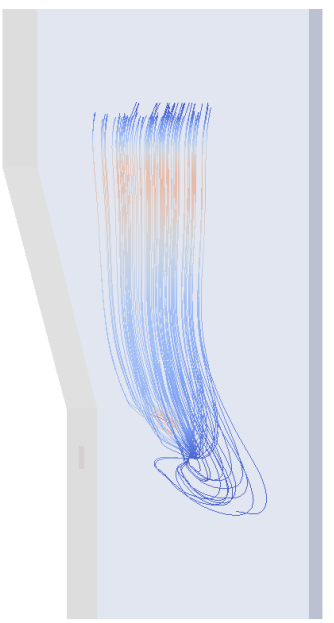

(g) $\mu_{s}=0.9$, stream lines

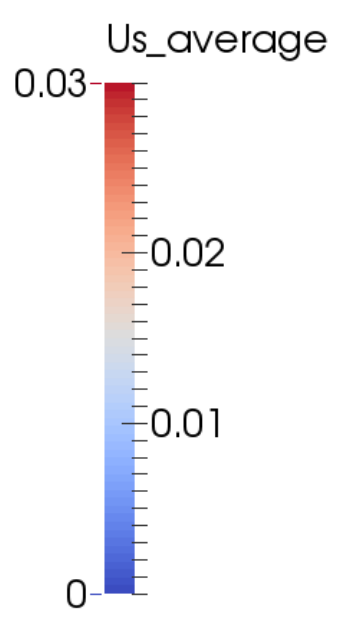

(d) Legend.

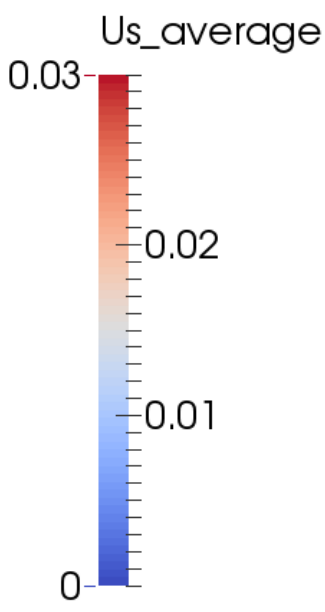

(h) Legend.

Figure 10.4: Vertical slice of particle velocity 1. The effect of sliding friction over the particle velocity is consistent. The area with fast particles is almost $50 \%$ larger in the low friction case.

We decided for this reason to represent each particle present in the domain slice considered in Fig. 10.5. As suggested by Hager [62], we colored differently the particles faster or slower than a series of fixed 
velocity thresholds. So, we were able to investigate to which extent from the inlet particles were set into motion.

It is clearly visible that the red, and faster, particles are in superior numbers for the low friction simulations. Further, in the high friction case, we can see in Fig. 10.5g that there are low velocities area even close to the raceway, condition not present in the low friction case.

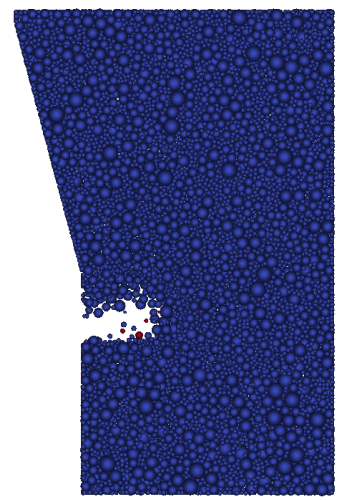

(a) $\mu_{s}=0.1, u_{l i m}=4 \mathrm{~m} / \mathrm{s}$

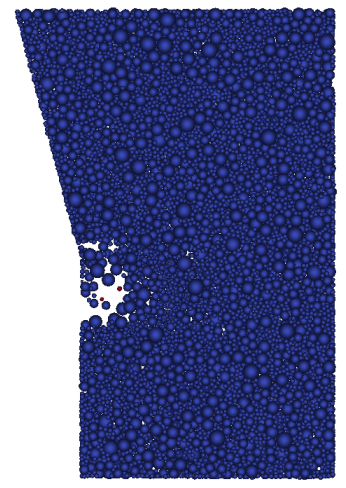

(e) $\mu_{s}=0.9, u_{\text {lim }}=4 \mathrm{~m} / \mathrm{s}$

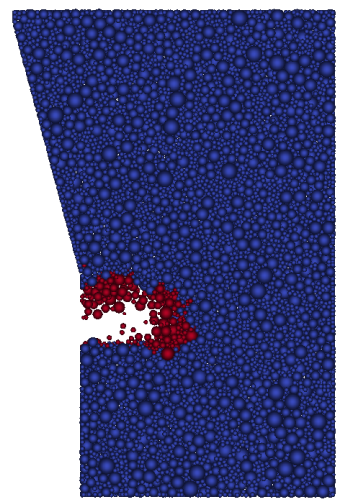

(b) $\mu_{s}=0.1, u_{\text {lim }}=0.1$ $\mathrm{m} / \mathrm{s}$

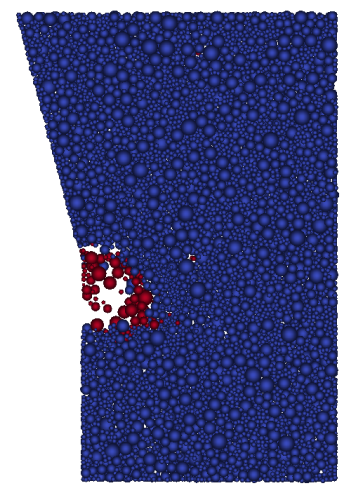

(f) $\mu_{s}=0.9, u_{l i m}=0.1 \mathrm{~m} / \mathrm{s}$

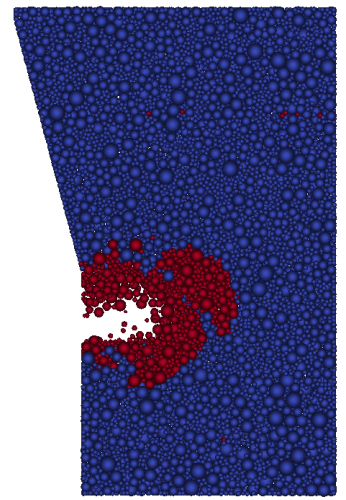

(c) $\mu_{s}=0.1, u_{\text {lim }}=0.01$ $\mathrm{m} / \mathrm{s}$

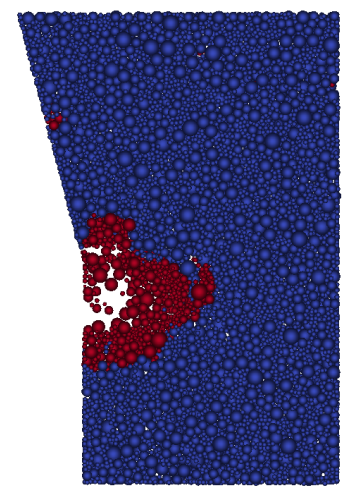

(g) $\mu_{s}=0.9, u_{l i m}=0.01$

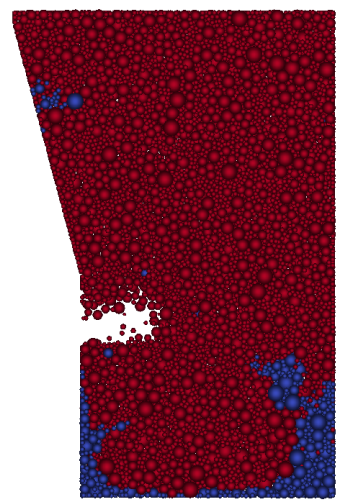

(d) $\mu_{s}=0.1, u_{l i m}=0.003$ $\mathrm{m} / \mathrm{s}$

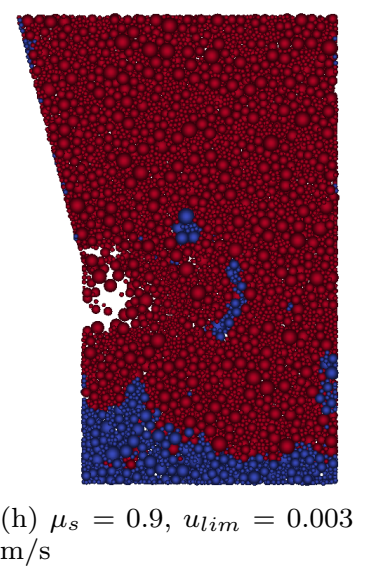

Figure 10.5: Vertical slice of particle velocity 2. Red particles are faster than the $u_{\text {lim }}$. The effect of sliding friction over the particle velocity is consistent. Many more particles show faster velocity than the threshold in every representation.

\subsubsection{Penetration depth}

Hager 62] calculates the raceway penetration depth with Merry's model:

$$
L=5.2 \cdot\left(\frac{\rho_{f} d_{T}}{\rho_{s} d_{p}}\right) \cdot 0.3 \cdot d_{T} \cdot\left(1.3 \cdot\left(\frac{u^{2}}{g d_{T}}\right)^{0.2}-1\right),
$$

with $d_{T}$ as the tuyere diameter, $d_{p}$ is the particle diameter, averaged in our case, $\rho_{f}$ and $\rho_{p}$ are the fluid and particle density, respectively. Of course, $u$ is the inlet velocity and $g$ represents the gravitational constant. Her results estimate a penetration depth of 0.8 meters.

Our results, shown in Fig. 10.6 were inferior to this estimation. Hager [62] suggested two main reasons for the discrepancy between CFD, DEM numerical results and theoretical analysis: a) only the particles 
with size over a defined diameter were modelled, and b) the void fraction inside the experimental setup is unknown and, thus, impossible to model reliably.

Especially, a) was due to computational considerations. Including in the particle distribution even the small particles led to an increase of the amount of particles to more than half a million. In addition, this brought the computational time for a single simulation with 32 cores to exceed a month duration. We attempted to reduce the simulation domain to keep the particle distribution and to consider at the same time a reduced amount of particles. Due to the unsufficient volume, the raceway could not properly develop, the results were inconclusive, and even channeling was observed. A complete discussion of this attempt is out of the scope of this thesis.

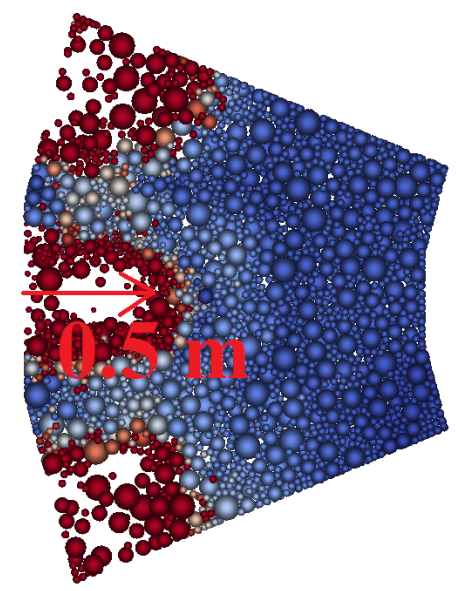

(a) $\mu_{s}=0.1$

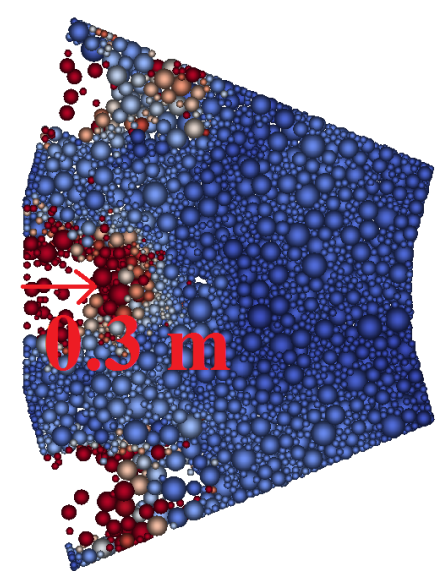

(b) $\mu_{s}=0.9$.

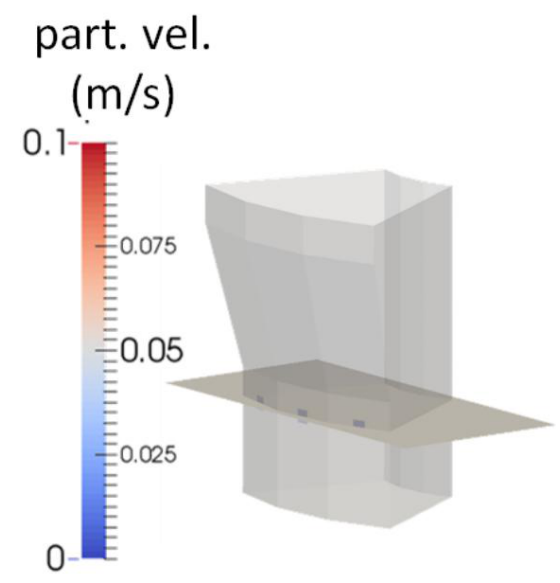

(c) Legend and slice position.

Figure 10.6: Raceway penetration depth on the horizontal. In the low friction case (Fig. 10.6a) the penetration depth is $65 \%$ larger than in the high friction simulation (Fig. 10.6b), showing the deep influence of this parameter even in large scale simulations. 
Part V

Conclusion 
We have presented a two-step method for DEM simulation parameter identification, and used the identified parameters for large scale numerical simulations. In the first step, an artificial neural network is trained using dedicated DEM simulations in order to predict bulk behaviours as function of a set of DEM]simulation parameters. In the second step, this artificial neural network is then used to predict the bulk behaviour of a huge number of additional DEM]parameter sets. The main findings of this study can be summarized as follows:

- An artificial neural network can be trained by a limited number of dedicated DEM simulations. The trained artificial neural network is then able to predict granular bulk behaviour.

- This prediction of granular bulk behaviour is much more efficient than computationally expensive DEM simulations. Thus, the macroscopic output associated with a huge number of parameter sets can be studied.

- If the predictions of the artificial neural network are compared to a bulk experiment, valid sets of DEM simulation parameters can be readily deduced for a specific granular material.

- This DEM parameter identification method can be applied to arbitrary bulk experiments. Combining two artificial neural networks which predict two different bulk behaviours leads to winnowing the set of valid DEM simulation parameters.

- In turn, an empty intersection between the networks would be a clear indication that the chosen contact law is not appropriate.

- Artificial neural networks can establish the complicated relationship between microscopic DEM parameters of a given contact law and macroscopic properties of granular materials with less DEM simulations than direct procedures.

- Additional numerical simulations for additional materials with different size distributions and stress conditions can be used to train additional ANNS These predict the numerical bulk for the investigated material. Again, confrontation with experimental measumerements provides valid microscopic values.

- The valid values obtained are used for a large scale simulation. It represents the movements of sinter particles along a chute. The simulation, tuned with the given parameters, successfully demonstrated the effectiveness of the chute as a segregation system.

- Similarly, the valid values for all the investigated materials were used in a blast furnace simulation. Especially, the raceway area was investigated. We evaluated the effect of the variation of the friction coefficient for the particle. The numerical results gave useful insights over the process. 
Appendix A

Powder Technology journal paper 


\title{
Identification of DEM simulation parameters by Artificial Neural Networks and bulk experiments
}

\author{
L. Benvenuti ${ }^{\mathrm{a}, *}, \mathrm{C}$ Kloss $^{\mathrm{b}}$, S. Pirker ${ }^{\mathrm{a}}$ \\ a Johannes Kepler University Linz, Department on Particulate Flow Modelling, Altenbergerstrasse 69, 4040 Linz, Austria \\ b DCS Computing GmbH, Altenbergerstr. 66a - Science Park, 4040 Linz, Austria
}

\section{A R T I C L E I N F O}

\section{Article history:}

Received 12 February 2015

Received in revised form 4 November 2015

Accepted 2 January 2016

Available online 11 January 2016

\section{Keywords:}

Discrete Element Method (DEM) simulations

Parameter identification

Artificial neural networks

\begin{abstract}
A B S T R A C T
In Discrete Element Method (DEM) simulations, particle-particle contact laws determine the macroscopic simulation results. Particle-based contact laws, in turn, commonly rely on semi-empirical parameters which are difficult to obtain by direct microscopic measurements. In this study, we present a method for the identification of DEM simulation parameters that uses artificial neural networks to link macroscopic experimental results to microscopic numerical parameters. In the first step, a series of DEM simulations with varying simulation parameters is used to train a feed-forward artificial neural network by backward-propagation reinforcement. In the second step, this artificial neural network is used to predict the macroscopic ensemble behaviour in relation to additional sets of particlebased simulation parameters. Thus, a comprehensive database is obtained which links particle-based simulation parameters to specific macroscopic bulk behaviours of the ensemble. The trained artificial neural network is able to predict the behaviours of additional sets of input parameters accurately and highly efficiently. Furthermore, this method can be used generically to identify DEM material parameters. For each set of calibration experiments, the neural network needs to be trained only once. After the training, the neural network provides a generic link between the macroscopic experimental results and the microscopic DEM simulation parameters. Based on these experiments, the DEM simulation parameters of any given non-cohesive granular material can be identified.
\end{abstract}

(c) 2016 Elsevier B.V. All rights reserved.

\section{Introduction}

Particles in various forms - ranging from raw materials to food grains and pharmaceutical powders - play a major role in a variety of industries. Discrete Element Methods (DEMs) are widely used to simulate particle behaviour in these granular processes [1].

In their original formulation of DEM, Cundall and Strack [2] allowed two particles to slightly overlap upon contact, and consequently they proposed repulsive forces in relation to this overlap distance. Their fundamental modelling concept has since been widely accepted in the literature and their soft-sphere contact law has been developed further by numerous researchers [3,4]. With increasing computational resources, $D E M$ simulations have become very popular giving rise to the development of commercial (e.g., PFC3D, used by Wensrich and Katterfeld [5]) and open-source software (e.g., LIGGGHTS, Kloss et al. [6], Aigner et al. [7]). Soft-sphere DEM simulations of thousands of particles have been proven to faithfully model particle bulk behaviour ([8]).

In these macroscopic DEM simulations, the contact law kernel between a pair of particles determines the global bulk behaviour of the granular material ([9]). As a consequence, defining a correct contact law is of crucial importance for the predictive capability of DEM simulations.

\footnotetext{
* Corresponding author.

E-mail address: luca.benvenuti@jku.at (L. Benvenuti).
}

Since DEM contact laws are based on a set of semi-empirical parameters, correct contact law parameters must be defined for a given granular material or DEM simulations will fail [10].

Identifying DEM contact law parameters is not a trivial task. Due to the huge number of particles in a granular material, it may be impractical to identify valid parameter sets by performing bilateral particle collision experiments. Furthermore, some contact law parameters such as the coefficient of rolling friction are purely empirical and cannot be determined by direct particle-to-particle measurements [5]. Therefore, DEM contact law parameters (Table 1 ) are commonly determined by comparing the macroscopic outcome of large-scale DEM simulations with bulk experiments [11]. If DEM simulation results disagree with bulk measurements, the set of contact law parameters must be adjusted until reasonable agreement is achieved. However, this purely forward methodology of parameter identification is limited by the multi-dimensionality of the parameter space and the associated computational costs of the required DEM test simulations. Moreover, one parameter set which is valid for one bulk behaviour (e.g., angle of repose) might fail for another (e.g., shear tester).

There are yet ways to determine contact parameters directly by measuring material properties or by performing particle based experiments, see e.g. Combarros et al. [10], Paulick et al. [12], and Lommen et al. [13]. Luding and Vidyapati $[14,15]$ are two of the most prominent examples of parameters identification by theoretical analysis. However, they focused on glass spheres, while non-sphericity was one of the fields we 
Table 1

DEM parameters. The upper parameters were identical in all simulations. The lower parameters were constant in each simulation, but were varied between simulations.

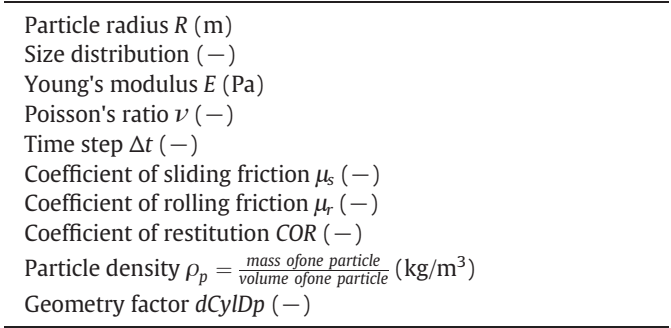

wanted to investigate. Further, it is related to all the microscopic parameters we analysed. Also the procedure of Combarros et al. [10], although on industrial samples (aluminium oxide), still had mostly spherical particles. In addition, these methodologies are laborious, since they have to be performed for every new granular material prior to a DEM simulations. Especially for the already cited rolling friction parameter, it is arduous to link the rolling friction parameter to the non-sphericity of the particle. Clearly, there is a need for an efficient method for identifying DEM contact law parameters, given a specific particle behaviour. In our study, we harnessed Artificial Neural Networks (ANNS) in order to reduce the number of $D E M$ test simulations required. ANNs have proven to be a versatile tool in analysing complex, non-linear systems of multi-dimensional input streams $[16,17,18]$. In our case, we fed an $A N N$ with DEM contact law parameters as input and compared the output with the bulk behaviour predicted by a corresponding DEM simulation. The difference between ANN prediction and DEM prediction is used to train our specific $A N N$ with a backward-propagation algorithm (described further below). After a training phase comprising a limited number of DEM test simulations, the ANN can then be used as a stand-alone prediction tool for the bulk behaviour of a granular material in relation to DEM contact law parameters. In this study, we applied this parameter identification method to two different granular bulk behaviours, namely the angle of repose $(A o R)$ test and the Schulze shear cell (SSC) test. In both cases, we first trained a specific ANN using a number of DEM test simulations before we identified valid sets of DEM contact law parameters by comparing the stand-alone ANN predictions with corresponding bulk experiments. For both cases we obtained valid sets of contact law parameters, which we then compared to formulate a reliable contact law for a given granular material. We further show that the same ANN can be used to characterize different granular materials, which have the same particle behaviour and can model with the same contact law.

In the next section we define some prerequisites including DEM contact law definitions, a general description of the ANN functionality, and the proposed method of DEM contact law parameter identification. We then describe how it is applied to characterize the DEM contact law parameters of sinter fines.

\section{DEM parameter identification}

Fig. 1 illustrates the methodology used.

\subsection{Applicability and scope}

The material used for this work was sinter fine, see Fig. 2. Its particles had a mean radius of $0.73 \mathrm{~mm}$ and were cohesionless, see Section 2.4. Their amounts of open and closed pores are not negligible (see [19]), and thus the use of an Archimedean procedure to determine the correct particle density by direct measurement was not applicable. Possibly, a tomography for each particle would lead to the particle density, but this analysis was infeasible: the necessary time for the amount of material analysed was unreasonable. Further, a three-dimensional scan of each particle would have surely physically identified our particles, and made us able to confront them with other studies. The high shape dispersity made also this analysis impossible. We followed a different procedure, see Section 2.4

To avoid the macroscopic plastic deformations analysed by Harthong et al. [20] we checked for each simulation for its total duration that the packing density was lower than a defined limit. This threshold (0.671) was given by the packing density of the close random packing with the same size distribution, as suggested and with the software provided by Baranau, Tallarek et al. [21,22]. The experimental characterization was performed at environmental temperature and thus temperature was not considered in the numerical simulations. Chemical reactions and aging were also not part of the scope of this work. Further, due to the limited number of experiments realized, a restricted number of flow regimes were tested, and only one size distribution was considered. The authors are confident that these two limitations have not compromised the validity of the method. Rather, further investigations on these two aspects could again demonstrate its effectiveness. Notably, we chose an elastic model to picture the particle behaviour. If we try to identify parameters for an elastic contact model in a system of nonelastic particles, this ANN approach will fail. So choosing appropriate contact models is an essential pre-requisite for contact parameter identification by means of ANN. Moreover, ANNs can give indications about the correctness of the chosen model, see Section 3.3.

\subsection{Discrete element method}

For each particle i inside the domain, a Discrete Element Method (DEM) code follows the trajectory and calculates the force that particle i exerts on particle $\mathrm{j}$, according to the imposed contact law. We decided to utilize a single contact law, equal for each particle, in all the simulations

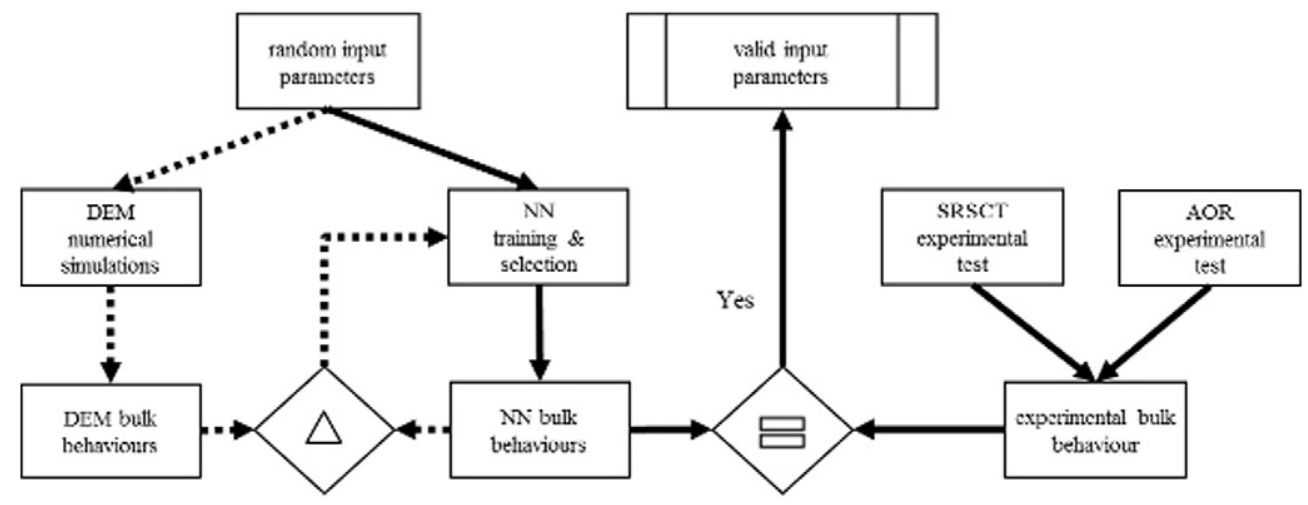

Fig. 1. Method. In the training phase (dashed lines) DEM simulations are performed with random initial input parameters. The behaviours obtained are used to train the Artificial Neural Networks (ANNs) in a loop that continues until the difference between the outputs of each ANN and its simulations is below the limit $(\Delta)$ (see Section 2.3 ). In the parameters identification phase (solid lines) we identify valid input parameters by comparing $(=)$ ANNs and experimental behaviours. Further explanations can be found in Section 2. 


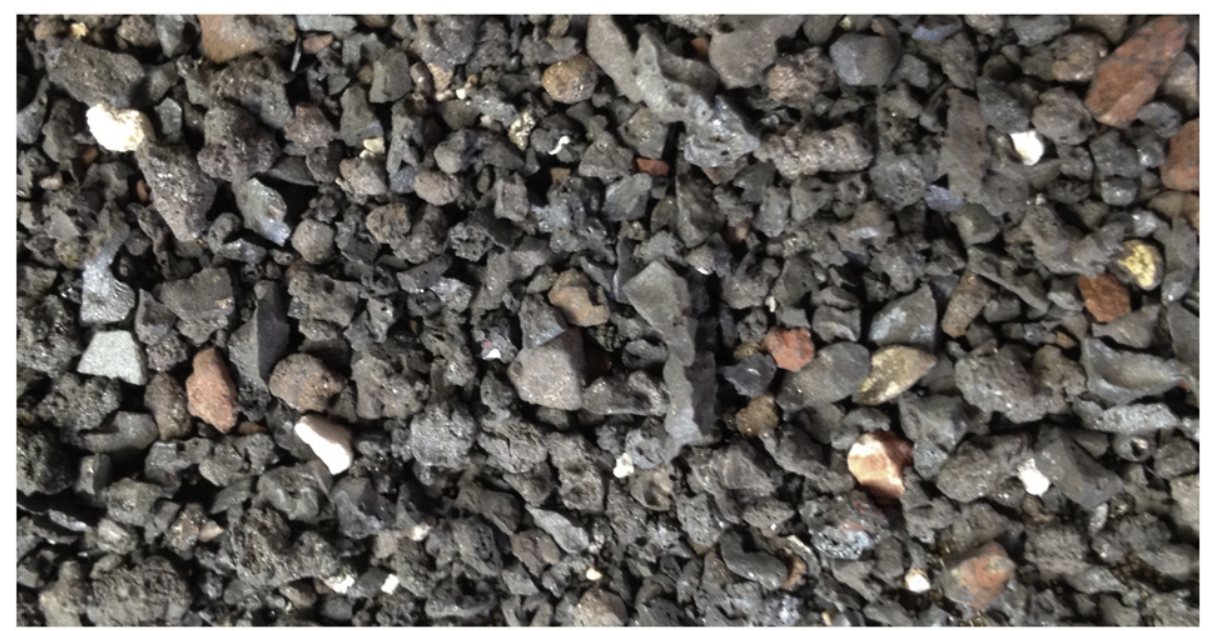

Fig. 2. Sinter ore fine analysed.

performed. Di Renzo and Di Maio [4] suggested using the elastic nonlinear Hertzian model without cohesion for the particle-particle and particle-wall contacts. LIGGGHTS, the simulation toolbox we used, meets all the requirements of modelling a shear tester, see Kloss et al. [6], Aigner et al. [7], and Benvenuti et al. [23]. The DEM parameters for the Young's modulus $(E)$ and the Poisson's coefficient $(\nu)$ were taken from the literature, see $[24,25]$; however we reduced the former to increase the time step $(\Delta t)$, following the recommendations of Ai et al. [9]. The time step was between $1.29 \%$ and $1.53 \%$ of the Rayleigh time, which also depends on the particle density $\left(\rho_{p}\right)$. Furthermore, we locked the size distribution, which was obtained by experimental sieving, see Table 2. In the contact law we used, the tangential component of the contact force between two generic particles $\left(F_{t}\right)$ is truncated to fulfil:

$$
F_{t} \leq \mu_{s} F_{n},
$$

where $F_{n}$ is the normal component and $\mu_{s}$ is the coefficient of sliding friction, one of the particle-based DEM parameter we investigated, another being the coefficient of rolling friction $\left(\mu_{r}\right)$. For coarse non-spherical particles, this is a critical parameter and describes inter-particle friction in medium to dense granular flow simulations. It is proportional to the torque counteracting the rotation of the particle. The $\mu_{r}$ parameter enters the equations according to the elasto-rolling resistance model presented by Wensrich and Katterfeld [5] and Ai et al. [9] based on the work of Jiang et al. [26]. The model is called EPSD2 in LIGGGHTS and is appropriate for both one-way and cyclical rolling cases. The maximum magnitude of rolling resistance torque is (Eq. (2)):

$T_{r \max }=\mu_{r} R_{r}\left|\widetilde{F}_{n}\right|$,

where $R_{r}$ is the equivalent radius and $F_{n}$ is the normal force. The last two particle-based DEM parameters we investigated were $\rho_{p}$ (see Section 2.1) and the coefficient of restitution (COR) as defined by Ai. et al. [9].

Although the geometry differs, the SSC was designed to obtain the same values for the shear stresses as the Jenike shear cell tester (JSCT), but with improved automation and reliability, see Schulze [27]. For this reason, the simulation setup was based on the JSCT. As suggested by Aigner et al. [7] and Benvenuti et al. [23], the diameter and the height of the rings operated in the simulations had to be sufficiently large to avoid relevant wall effects. However, a larger domain increases the

Table 2

$D E M$ fixed input values.

\begin{tabular}{lllll}
\hline $\begin{array}{l}\text { Mean } R \\
(\mathrm{~mm})\end{array}$ & $\begin{array}{l}\text { Std.dev. } R \\
(\mathrm{~mm})\end{array}$ & $\begin{array}{l}\text { Young's modulus } \\
(\mathrm{MPa})\end{array}$ & $\begin{array}{l}\text { Poisson's ratio } \\
(-)\end{array}$ & $\begin{array}{l}\Delta t \\
(\mathrm{~s})\end{array}$ \\
\hline 0.732 & 0.41 & 10 & 0.40 & $10^{-6}$ \\
\hline
\end{tabular}

number of particles and thus simulation time. For this reason, we considered the cylinder dimension ( $d C y l D p)$, which is proportional to the mean particle diameter, as an additional DEM parameter to be investigated. A simulation run comprised four phases. First, the shear cell was filled with the granulate material, and it was allowed to settle. Then, the top lid was lowered and the first normal stress applied to the bulk solid. The distance between the lid and the bottom of the domain multiplied by the simulation area gave the total volume, while the software already provided the total mass. The velocity of the ring displacement, and consequently the total simulation time, was determined by a trade-off between minimizing the normal load oscillation and optimizing computation time. The former requires (relatively) low velocity, and the latter requires high speed.

The coefficients, COR, $\mu_{s}, \mu_{r}, \rho_{p}$ and $d C y l D p$ (the cylinder dimension, proportional to the mean particle diameter), as indicated in Table 4, were constant in each simulation, but their combination differed between simulations. Further, $d C y l D p$ was used to evaluate the wall effect, but only $10 \%$ of the simulations had a dCylDp larger than 20 . The normal stress $\sigma_{n}(1068,2069$, and $10,070 \mathrm{~Pa})$ and its percentage $(40,60,80$, and $100 \%$ ) during the incipient flow condition $\left(\tau_{\%}\right)$ varied to replicate twelve shear-cell load conditions. A Matlab script allowed us to extract from the simulation output the numerical values representative of bulk behaviour (hereafter called bulk values), see Table 3, for each DEM simulation parameter combination. For correctly performed simulations, we observed a stress path as in Fig. 3b. First, the $\sigma_{n}$ was kept constant while the coefficient of internal friction $\left(\mu_{i e}\right)$ initially increased and then reached a plateau. The second bulk value $\left(\mu_{p s h}\right)$ was calculated as the average of the $\mu_{i e}$ in this plateau. The $\sigma_{n}$ was then automatically reduced, in our example to $80 \%$ of its initial value. Subsequently, a second

Table 3

Values representative of bulk behaviour.

\begin{tabular}{lll}
\hline & Acronym & Formula \\
\hline Bulk density & $\rho_{b}$ & $\frac{\text { mass of the bulk }}{\text { volume of the bulk }}$ \\
$\begin{array}{l}\text { Steady-state flow/pre-shear coefficient of } \\
\text { internal friction }\end{array}$ & $\mu_{p s h}$ & $\frac{\tau_{p s h}}{\sigma_{n, p s h}}$ \\
$\begin{array}{l}\text { Incipient flow/shear coefficient of internal } \\
\text { friction }\end{array}$ & $\mu_{s h}$ & $\frac{\tau_{s h}}{\sigma_{n s h}}$ \\
Static angle of repose & AOR & From the slope \\
\hline
\end{tabular}

Table 4

DEM variable input values for training the Artificial Neural Networks.

\begin{tabular}{lllll}
\hline$\mu_{s}(-)$ & $\mu_{r}(-)$ & $\operatorname{COR}(-)$ & $\rho_{p}\left(\mathrm{~kg} / \mathrm{m}^{3}\right)$ & $\operatorname{dCylDp}(-)$ \\
\hline $0.4 / 0.6 / 0.8$ & $0.4 / 0.6 / 0.8$ & $0.5 / 0.7 / 0.9$ & $2500 / 3000 / 3500$ & $20 / 36 / 38 / 40$ \\
\hline
\end{tabular}




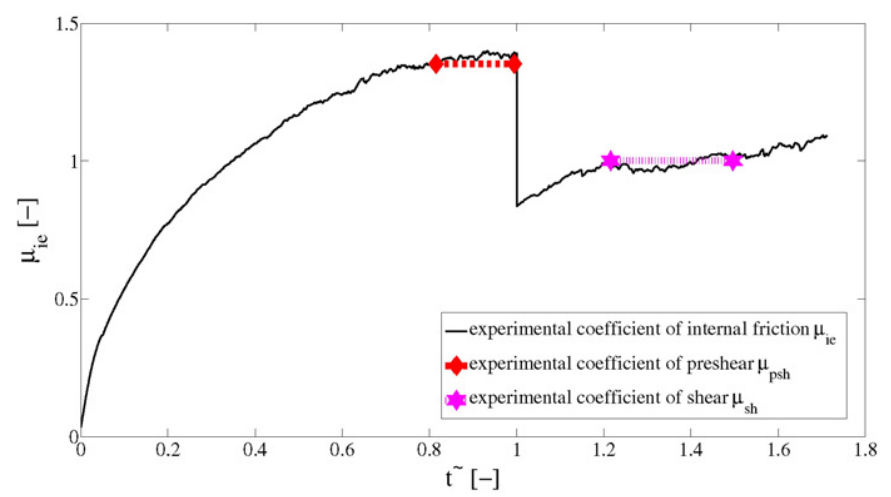

(a) Experimental shear-cell tester stress path $-\sigma_{n}=10000 \mathrm{~Pa}$

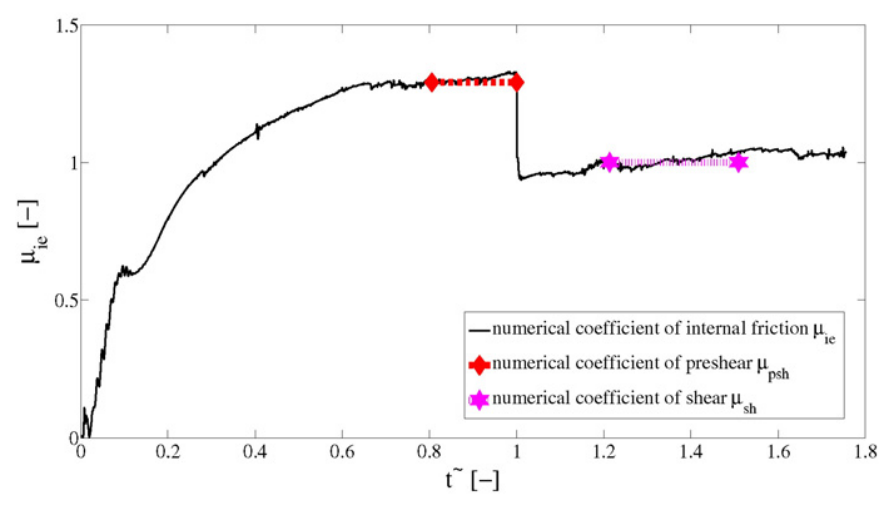

(b) Numerical shear-cell tester stress path $-\sigma_{n}=10000 \mathrm{~Pa}$

Fig. 3. Experimental and numerical samples of the stress path for the Schulze ring shear cell tester. Time was normalized: $t \sim=t / t_{\text {change, }}$, where $t_{\text {change }}$ is the point in time at which the normal stress $\left(\sigma_{n}\right)$ was modified during the tests. Until $t \sim=1$, the $\sigma_{n}$ was kept constant at 10,000 Pa. In Panel a, a plateau was reached at $t \sim=0.91$. The coefficient of pre-shear $\left(\mu_{p s h}\right)$ was calculated as the average of the coefficient of internal friction $\left(\mu_{i e}\right)$ in this first plateau. At $t \sim=1$, the $\sigma_{n}$ was reduced to $80 \%$ of its initial value, and soon after a second plateau developed. We obtained the coefficient of shear $\left(\mu_{\mathrm{sh}}\right)$ as the average of $\mu_{i e}$ in this second plateau. The stress paths agree well, especially the plateaux. They were clearly relevant because the values representative of the bulk behaviours were collected there.

plateau developed. We obtained the third value $\left(\mu_{\text {sh }}\right)$ as the average of $\mu_{i e}$ in this second plateau. The stress path accords with the experimental one, especially the plateaux.

In AoR simulations we sought to replicate meticulously the experimental setup, considering both the plate and the liftable boundary, with the same domain size consideration as before. The particles had the same properties as in the shear-cell simulation. The first phase was identical to that of the shear-cell simulation. After lifting the boundary, the particles formed a heap. An image post-processing software was used to obtain the average slope, the fourth bulk value, allowing us to define the numerical bulk behaviour.

\subsection{Artificial Neural Networks}

An Artificial Neural Network (ANN) is a powerful modelling technique, that is based on non-linear functions ([18]). In this work, we first used the ANN to fit the DEM numerical simulation data, and then to process a vast number of parameters combinations. ANNs map combinations of input data to convenient outputs (fitting). Especially, the validity of the Feedforward Multilayer Perceptron Neural Network (MLPNN) with a backpropagation reinforcement learning training algorithm (scaled conjugate gradient), has been demonstrated in the literature, see Haykin [18]. Several scientists [28-35] have employed ANNs to model the mechanical properties of materials. Following the best practice suggested by Vaferi et al. [16], we used MLPNNs.

Our ANNs have three different layers: the input layer has a number of neurons equal to the number of different inputs of the network, see Fig. 4. The hidden (or central) layer's number of neurons was to be investigated. The output layer contains one neuron for the output. The transfer functions between the first two layers are the tangential sigmoid, and those between the hidden and output layers are linear.

Thus, we were able to use the DEM parameter combinations and their corresponding bulk values to train the ANNs. Note that $15 \%$ of the simulations (test simulations) were randomly picked and excluded from the training processes. We started with all the DEM parameter combinations and their corresponding numerical $\mu_{p s h}$ to create 36 ANNs that differed in their numbers of neurons in the hidden layer (between five and forty neurons). We then determined the coefficient of determination $\left(R^{2}\right)$ between the bulk-macro behaviours in the output of the ANN and the 15\% test simulations, which were not correlated with the remaining $85 \%$ used for the training. Thus, we could select for $\mu_{p s h}$ the ANN with the maximum $R^{2}$, again as suggested by Vaferi et al. [16], and we noted its number of
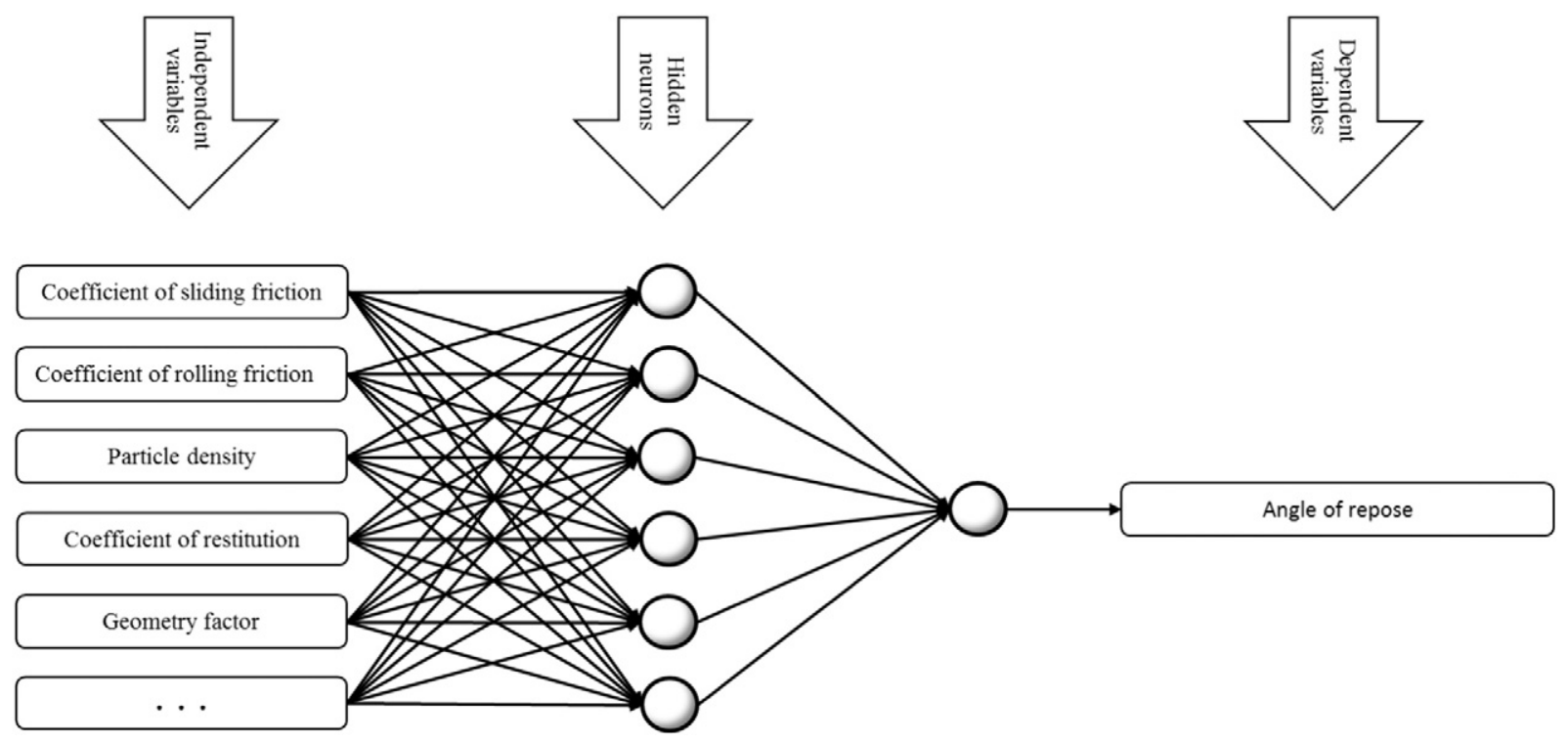

Fig. 4. Artificial Neural Network (ANN) Scheme of how the Multilayer Perceptron ANN (MLPNN) derives one bulk-behaviour-dependent variable from the mutually independent simulation variables. 
neurons. We repeated the same $A N N$ creation steps for $\mu_{s h}, \rho_{b}$ and $A o R$, obtaining one trained $A N N$ for each bulk value.

Since $\mu_{p s h}, \mu_{s h}$ and $\rho_{b}$ belonged to the shear-cell simulations, their ANNs were handled together: we had one cluster with three ANNs for the shear cell and one with only one ANN for the AoR. We could then proceed in identifying valid input parameters. Oberkampf et al. [36] suggested using a Design of Experiments (DoE) method to determine the parameter combinations to be simulated. They stated that this approach allows optimization of computation time with an acceptable loss of precision. Further, the quality of the ANN data had to be examined critically. Haykin [18] suggested considering the quality of (a) ANN training process and (b) the subsequent data generation based on the inputs provided. Task (a) is particularly important when dealing with experimental training data, and usually addressed by noise-corrupted pattern calibration. However, our training pool was numerical and extensive, and the particles in our simulations were inserted using a random seed value. For vast amounts of training data the effect of noise-corrupted patterns is negligible, see Haykin [18]. Thus, in our work task (b) was more challenging. Once trained, the ANNs were fed combinations of DEM parameters.

Instead of following Oberkampf's suggestion, the speed of the trained ANNs enabled us to follow a different approach in maximizing the precision of the characterization and in increasing the generalization. We created random values in the range and numbers defined in Table 5 according to a standard uniform distribution for each parameter investigated. The total number of combinations of these random values was $6,250,000$. These combinations were then fed to and processed by the selected ANNs, and thus three bulk values for the shear cell and one for the AoR were obtained. At this point we could use the ANNs as a tool to deliver parameters for the specific contact law, which was not questioned further. However, if the contact law was not appropriate this methodology might help in identifying the DEM deficiencies related to it.

\subsection{Macroscopic experiments and parameter identification}

A representative sample of bulk solid was placed in a Schulze ring shear cell (SSC) of specified dimensions (externalradius $=100 \mathrm{~mm}$, internalradius $=50 \mathrm{~mm}$ ), see Schulze [27]. Once checked that the material was cohesionless, we obtained for each of the twelve load conditions of the SSC three bulk values $\left(\mu_{p s h}, \mu_{s h}\right.$ and $\left.\rho_{b}\right)$.

Later, a sample was deposited on a $20 \mathrm{~cm}$ diameter plate with liftable boundary called static angle of repose $(A o R)$ tester. Once the particles were in position, the boundary was lifted, allowing some particles to

Table 5

DEM random input values. Within each range the indicated number of random values was chosen according to a standard uniform distribution.

\begin{tabular}{lllll}
\hline & $\mu_{s}(-)$ & $\mu_{r}(-)$ & $\operatorname{COR}(-)$ & $\rho_{p}\left(\mathrm{~kg} / \mathrm{m}^{3}\right)$ \\
\hline Range & {$[0.1 \ldots 1.0]$} & {$[0.1 \ldots 1.0]$} & {$[0.5 \ldots 0.9]$} & {$[2000 \ldots 3500]$} \\
Number of values & 100 & 100 & 25 & 25 \\
\hline
\end{tabular}

\section{Table 6}

Values of linear relationship between variables considered multiplied by 100 . Sliding friction $\left(\mu_{\mathrm{s}}\right)$, rolling friction $\left(\mu_{r}\right)$ and particle density $\left(\rho_{p}\right)$ had the greatest influence on, respectively, the coefficient of pre-shear $\left(\mu_{p s h}\right)$, the angle of repose $(A o R)$ and the bulk density $\left(\rho_{b}\right)$. Notably, $\rho_{p}$ was not used as a training parameter for AoR bulk behaviour.

\begin{tabular}{lrrrrrrrr}
\hline & \multicolumn{1}{l}{$\mu_{s}$} & \multicolumn{1}{c}{$\mu_{r}$} & \multicolumn{1}{c}{ COR } & \multicolumn{1}{l}{$\rho_{p}$} & \multicolumn{1}{l}{$\mu_{\text {sh }}$} & \multicolumn{1}{c}{$\mu_{p s h}$} & \multicolumn{1}{l}{$\rho_{b}$} & \multicolumn{1}{l}{$A o R$} \\
\hline$\mu_{s}$ & 100.00 & 0.55 & 0.04 & 0.00 & 3.84 & 87.26 & 8.39 & 49.48 \\
$\mu_{r}$ & 0.55 & 100.00 & 0.15 & 0.00 & 58.92 & 33.70 & 3.10 & 60.20 \\
COR & 0.04 & 0.15 & 100.00 & 0.00 & 15.52 & 0.57 & 1.71 & 21.35 \\
$\rho_{p}$ & 0.00 & 0.00 & 0.00 & 100.00 & 4.98 & 5.71 & 99.00 & 0.00 \\
$\mu_{s h}$ & 3.84 & 58.92 & 15.52 & 4.98 & 100.00 & 26.03 & 9.52 & 0.00 \\
$\mu_{p s h}$ & $\mathbf{8 7 . 2 6}$ & 33.70 & 0.57 & 5.71 & 26.03 & 100.00 & 4.33 & 0.00 \\
$\rho_{b}$ & 8.39 & 3.10 & 1.71 & $\mathbf{9 9 . 0 0}$ & 9.52 & 4.33 & 100.00 & 0.00 \\
AoR & 49.48 & $\mathbf{6 0 . 2 0}$ & 21.35 & 0.00 & 0.00 & 0.00 & 0.00 & 100.00 \\
\hline
\end{tabular}

Bold values represent the most relevant micro-macro correlations.
Table 7

Experimental results. Values for three load conditions.

\begin{tabular}{llllll}
\hline$\sigma_{n}(\mathrm{~Pa})$ & $\tau(\mathrm{Pa})$ & $\mu_{p s h}(-)$ & $\tau_{\%}(\%)$ & $\mu_{s h}(-)$ & $\rho_{b}\left(\mathrm{~kg} / \mathrm{m}^{3}\right)$ \\
\hline 1068 & 1059 & 0.9916 & 80 & 1.2333 & 1718 \\
2069 & 1818 & 0.8787 & 80 & 0.9994 & 1759 \\
10070 & 8232 & 0.8175 & 80 & 1.1712 & 1802 \\
\hline
\end{tabular}

drop. Once stabilized, the $A o R$ was measured eight times using a digital protractor at different positions of the heap. The average of the measurements gave the fourth bulk value. Note that, since the experiments were performed only for larger-size bulk solids, the compaction condition in the initial state was not critical to the final result.

Subsequently, we compared the ANN and experimental bulk behaviours for the twelve shear-cell load conditions. If in a DEM-parameter combination all the three bulk values differed by less than $5 \%$ from those of the corresponding experiments, i.e.:

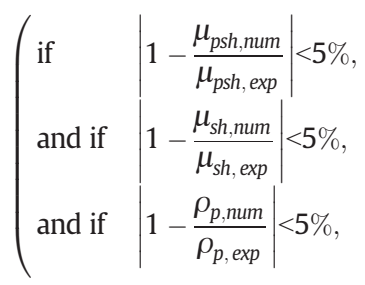

the combination was marked. The marked combinations were processed by the AoR ANN, and then compared with the experiment. Were considered valid those that differed by less than $5 \%$ also in this comparison (Eq. (4)):

if $\left|1-\frac{A o R_{\text {num }}}{A 0 R_{\exp }}\right|<5 \%$.

These parameters are to be considered valid for the defined contact law (and flow regime). Further, to prove the validity of the system, we tested the marked combinations by modifying the experimental bulk values of the shear cell. We artificially decreased or increased the shear force, and thus $\mu_{p s h}$ and $\mu_{s h}$, by a product coefficient $(P)$, e.g. Eq. (5):

$\mu_{\text {psh,new }}=\mu_{\text {psh,old }} \cdot P$.

Table 8

Valid DEM values. For each parameter we show the valid parameter statistics in the two tests and in their intersection. Finally, we show the number of valid parameter combinations over the total $(6,250,000)$.

\begin{tabular}{lllll}
\hline & Type & SSC & AoR & SSC \& AoR \\
\hline$\mu_{\mathrm{s}}(-)$ & Mean & 0.831 & 0.177 & 0.664 \\
& Std. dev. (SD) & 0.097 & 0.095 & 0.029 \\
& Range $(R)$ & 0.9 & 0.9 & 0.9 \\
$\mu_{r}(-)$ & SD/R & 0.108 & 0.106 & 0.032 \\
& Mean & 0.692 & 0.830 & 0.916 \\
& Std. dev. (SD) & 0.215 & 0.193 & 0.042 \\
& Range $(R)$ & 0.9 & 0.9 & 0.9 \\
COR $(-)$ & SD/R & 0.239 & 0.214 & 0.046 \\
& Mean & 0.708 & 0.590 & 0.590 \\
& Std. dev. (SD) & 0.104 & 0.073 & 0.065 \\
& Range $(R)$ & 0.4 & 0.4 & 0.4 \\
$\rho_{p}\left(\mathrm{~kg} / \mathrm{m}^{3}\right)$ & SD/R & 0.259 & 0.183 & 0.161 \\
& Mean & 2245.7 & 3192.8 & 2283.9 \\
& Std. dev. (SD) & 80.5 & 277.4 & 67.1 \\
& Range $(R)$ & 1500 & 1500 & 1500 \\
Valid combinations & SD/R & 0.054 & 0.185 & 0.045 \\
& Number & 290203 & 816552 & 3884 \\
& (\%) & 4.64 & 13.06 & 0.06 \\
\hline
\end{tabular}




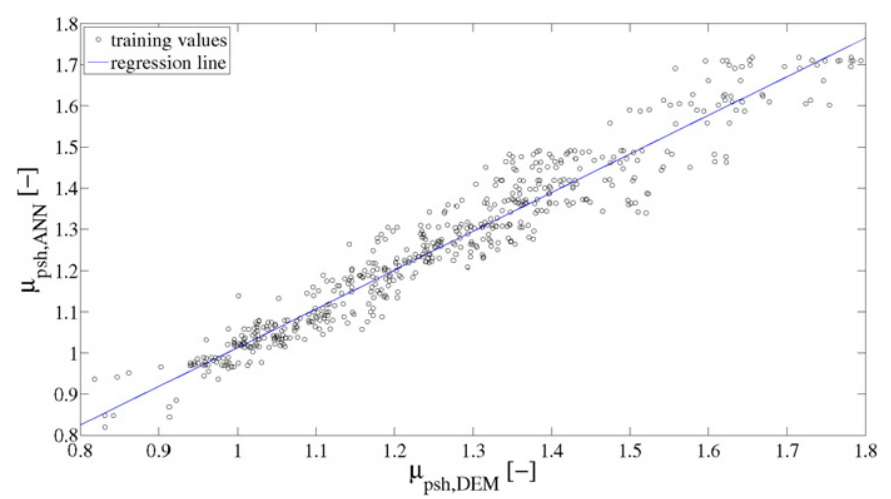

Fig. 5. Comparison between prediction of the trained Artificial Neural Network (ANN) and 546 full DEM simulations of the coefficient of pre-shear $\left(\mu_{p s h}\right)$. In this case the regression line is nearly linear (0.94), and demonstrates the accurate predictive power of the ANN.

\section{Results}

\subsection{DEM simulations}

For sinter fine, 546 shear cell and 81 static AoR simulations were run with the parameter combinations described in Table 4. The computational time amounted to $1 \mathrm{~h}$ with 32 AMD cores for a benchmark shear-cell simulation and to $9 \mathrm{~h}$ for a benchmark $A o R$ simulation, both with 50,000 particles. Simulations with larger $d C y l D p$ required more time (e.g., about $12 \mathrm{~h}$ for the shear cell with 400,000 particles).

\subsection{ANN model development}

First, we determined the regression of the bulk behaviour parameters, for instance the $\mu_{p s h}$; see Fig. 5 , where the corresponding plot for the $A N N$ with the maximum $R^{2}$ is shown. Each circle represents one of the 546 simulations. The plot shows a consistent agreement between the DEM and the $A N N$ values and an almost linear regression $\left(R^{2}=\right.$ $0.94)$. The linear relationship between the training values can be seen in Table 6. The clearest connections were between $\mu_{s}$ and $\mu_{p s h}$, and $\rho_{p}$ and $\rho_{b}$. In contrast, for $\mu_{s h}$ and $A o R$, the $\mu_{r}$ balanced the influence of the $\mu_{s}$.

We then investigated how the $R^{2}$ changed with the number of neurons for the $\mu_{p s h}$. In this case, we achieved a $R^{2}=0.96$ for an $A N N$ with fifteen neurons. Increasing the number of neurons did not improve the $R^{2}$; it even started to oscillate with higher numbers of neurons. We subsequently obtained the optimal number of neurons for all ANNs. Further, we processed the random combinations (Table 4) with the ANN. The ANN evaluation was significantly faster than the DEM simulations. The individuation of the numerical bulk behaviours for all the DEM combinations did not take more than a few seconds on a single core.

\subsection{Experiments and parameter identification}

Experimental values identifying the bulk behaviour, $\mu_{p s h}, \mu_{s h}$ and $\rho_{b}$, of sinter fine were acquired through SSC tests. Table 7 presents these values for three load conditions: clearly the $\mu_{p s h}$ decreases, and the $\mu_{s h}$ oscillates. The $\rho_{b}$ has a clear average of $1,760 \mathrm{~kg} / \mathrm{m}^{3}$ with a $42 \mathrm{~kg} / \mathrm{m}^{3} \mathrm{de}-$ viation. The stress path for the second load condition in Table 7 is shown in Fig. 3a. Two AoR tests were performed that gave an average angle of $38.85^{\circ}$. We obtained the radius $(R)$ mean and standard deviations, as shown in Table 2, from sieving experiments. Initially, we investigated the comparison between numerical and experimental behaviours for one load condition of the shear cell $\left(\sigma_{n}=1068 \mathrm{~Pa}, \mathrm{P}=1.0\right)$, as plotted in Fig. 6. Later, the comparison between numerical and experimental behaviours led to a first series of marked combinations (MC1) for one load condition of the shear cell $\left(\sigma_{n}=10,070 \mathrm{~Pa}, \mathrm{P}=1.0\right)$, as plotted in Fig. $7 \mathrm{~b}$, where the minimum and maximum values are shown, together with the mean. Note that the confidence interval is large, especially for the COR, which highlights its insignificant influence on the characterization. Compared to the smaller normal load, both the $\rho_{p}$ and the $\mu_{s}$, however, show a narrow confidence interval, which demonstrates their influence and the ability of this procedure to find valid DEM parameters. These results agree with our examination of the ratio of the standard deviation to the range, see Table 8 . Further, we observed that various DEM parameter combinations could reproduce the experimental behaviour, and thus we evaluated their mutual dependencies. This is shown more

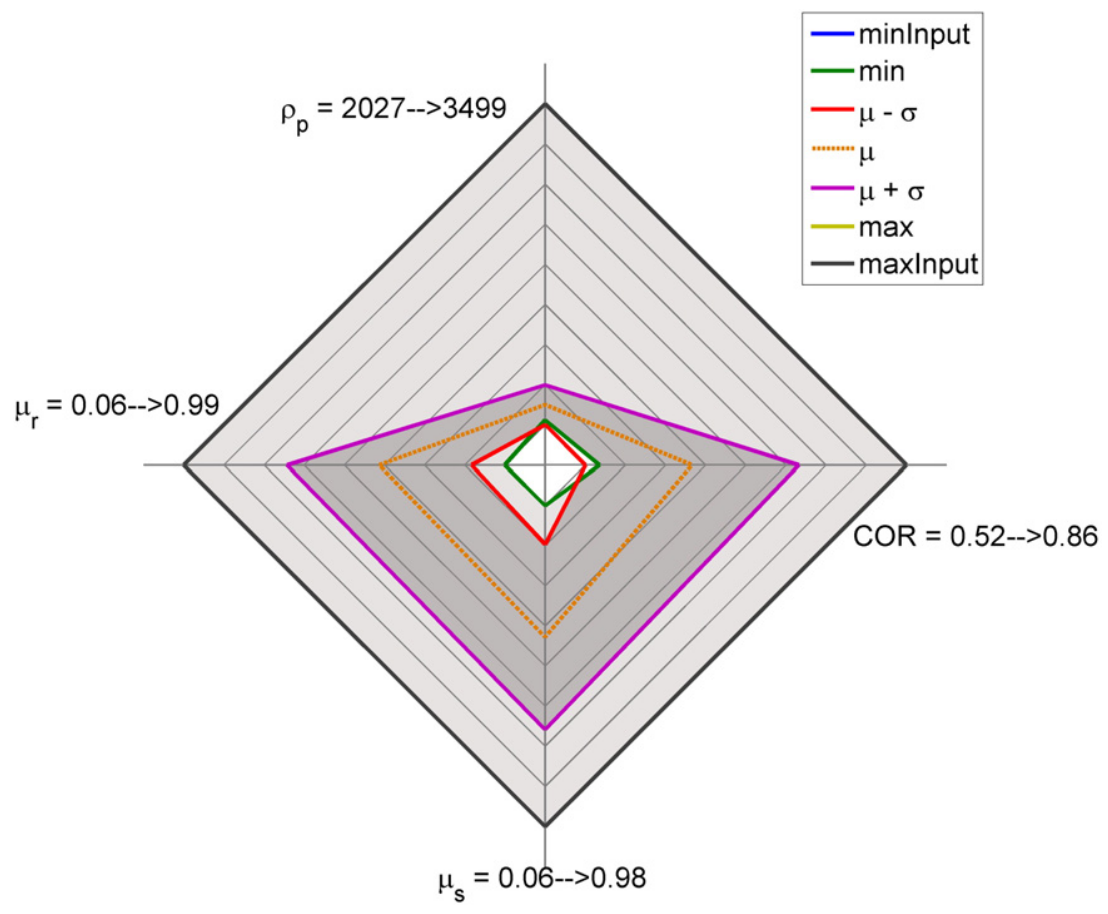

Fig. 6. Parameter space plot of valid simulation parameters for one bulk behaviour measured by a shear cell tester (SSC). Each axis of the parameter space plot represents one simulation parameter. The shaded area indicates valid parameter combinations, and dark shaded values indicate the confidence range. The marked combinations for $\sigma_{n}=1068$ Pa are presented. Further explanations can be found in Section 3.3. 


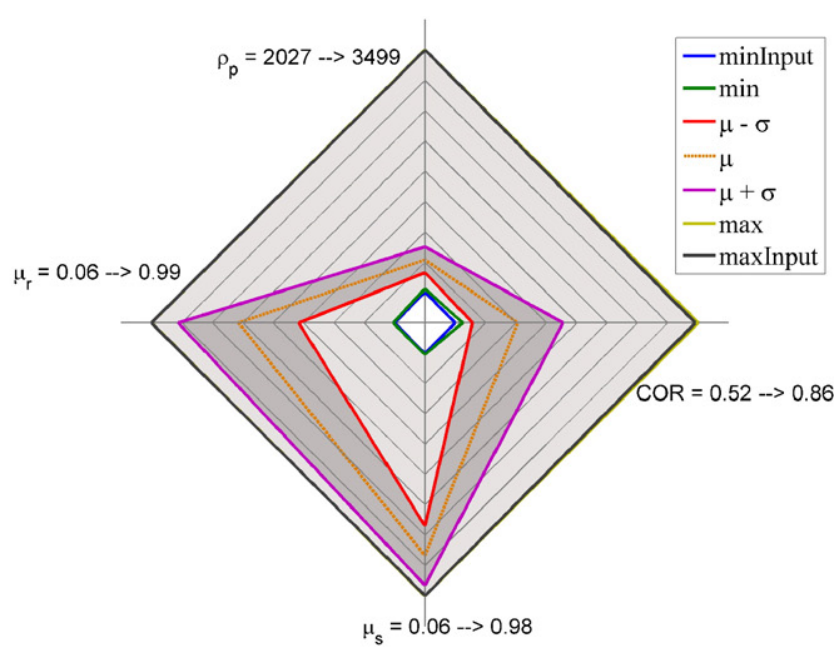

(a) Parameter space plot, $S S C, \sigma_{n}=$ 10, $070 \mathrm{~Pa}, \mathrm{P}=0.8$

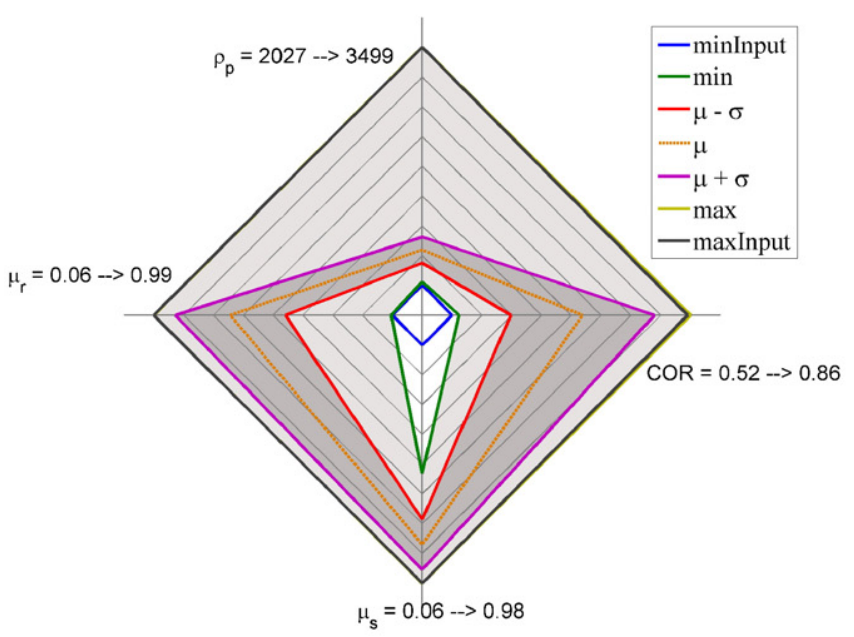

(b) Parameter space plot, $S S C, \sigma_{n}=$ 10, $070 \mathrm{~Pa}, \mathrm{P}=1.0$

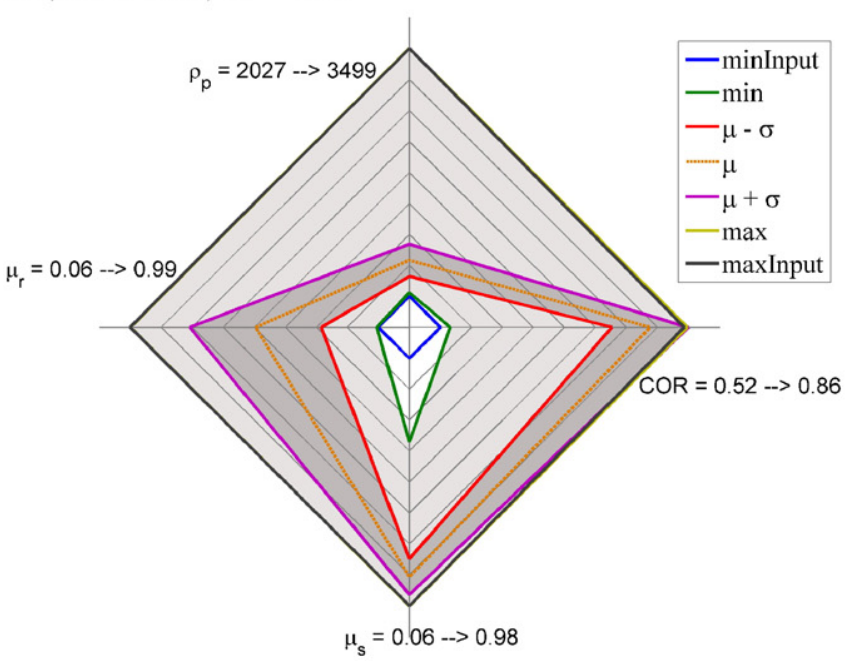

(c) Parameter space plot, $S S C, \sigma_{n}=$ 10, $070 \mathrm{~Pa}, \mathrm{P}=1.2$ clearly in a density plot (see Fig. 8b for MC1) of the particles' coefficient of restitution (COR) in relation to the coefficients of sliding friction $\left(\mu_{s}\right)$ and rolling friction $\left(\mu_{r}\right)$. Multiple combinations $(250,407$ or $4 \%$ of the total) of $\mu_{s}$ and $\mu_{r}$ reproduced the experimental behaviour with varying COR. This underlines once more their correlation, as already stated by Wensrich and Katterfeld [5]. Compared to the $\sigma_{n}=1068 \mathrm{~Pa}, \mathrm{P}=1.0$, we cannot claim that is in general possible to extrapolate information to other flow regimes: the parameters sets obtained are slightly different. Especially, the parameter combinations valid for both sets are unrealistically limited (less than one hundred). This, in turn, gives an indication that the functional particle behaviour, we have chosen for the DEM simulations is not able to correctly predict both macroscopic flow regimes. However, in this case we can argue that our ANN parameter identification approach can help in evaluating the predictive capability of a defined functional particle behaviour. To further demonstrate the validity of the procedure, we modified the product coefficient for $\sigma_{n}=10,070 \mathrm{~Pa}$. First, we set it to $P=0.8$, and we obtained another series of marked combinations (MC2). It can be seen in the parameter space plot in Fig. 7a that the confidence range is narrower than for $P=1.0$, while in the density plot in Fig. 8a the area appears larger, although slightly less densely populated. Finally, for $P=1.2$ and its marked combinations (MC3) the parameter space plot in Fig. 7c shows a largely different confidence range, while the density plot in Fig. 8c shows a smaller area. As expected, the procedure was highly sensitive to variations in the experimental data. Our approach could therefore be used in specific flow regimes for a range of bulk materials that can be represented with a defined particle bulk behaviour.

We then processed the random combinations with the AoR ANN. In Fig. 9a the parameter space plot for the same criteria as before can be seen. In accordance with theory ([5]), in a simulation dominated by rolling particles, the coefficient of rolling friction has the maximum influence. Finally, we extracted from the MC1 $\left(\sigma_{n}=10,070 \mathrm{~Pa}, \mathrm{P}=1.0\right)$ values the AoR ANN behaviour and compared it with the experimental one. As can be seen in the parameter space plot in Fig. 9b, the confidence interval is very small, indicating that all the parameters but the COR played an important role, and demonstrating the reliability of these parameter combinations in representing the bulk behaviour. From the initial 6,250,000 combinations, only 3884 were valid $(0.0621 \%)$, see Table 8.

\section{Discussion of model limitations}

In our approach we established a relationship between particle scale contact law parameters and macroscopic bulk behaviour by means of $A N N$. In case the chosen contact law is physically correct by its functional dependencies, this micro to macro relationship can be used in a reversed way to identify valid sets of contact law parameters.

However, in case the chosen contact law is not applicable to the granular material under consideration, our procedure might lead to a wrong set of parameters, which might lead to the correct bulk behaviour. In this case a first error (incorrect contact law) might be annulled by another one (wrong parameters).

To avoid such misleading results, the functional dependency of the particle based contact law should be chosen with care, taking into account the contact physics in the granular flow regime under investigation. Further, our ANN based parameter identification should be applied to different macroscopic bulk behaviours. If those parameter

Fig. 7. Parameter space plot of valid simulation parameters for three different bulk behaviours measured by a shear cell tester (SSC). Each axis of the parameter space plot represents one simulation parameter. The shaded area indicates valid parameter combinations, and dark shaded values indicate the confidence range. The marked combinations for $\sigma_{n}=10,070 \mathrm{~Pa}$ are presented. Further explanations can be found in Section 3.3. 

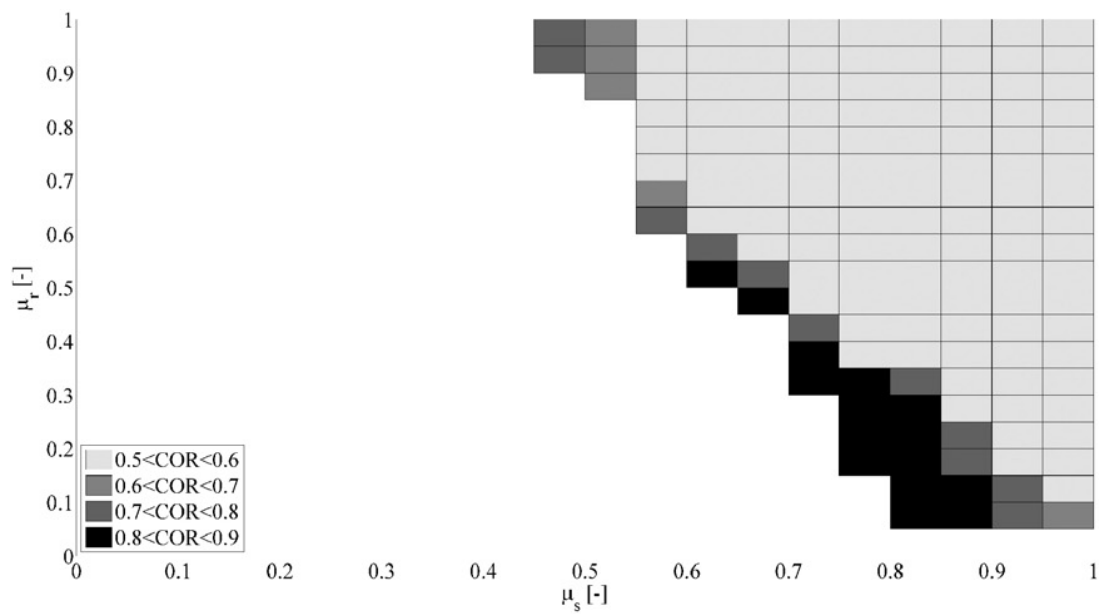

(a) Density plot, $S S C, \sigma_{n}=10,070 \mathrm{~Pa}, \mathrm{P}=0.8$

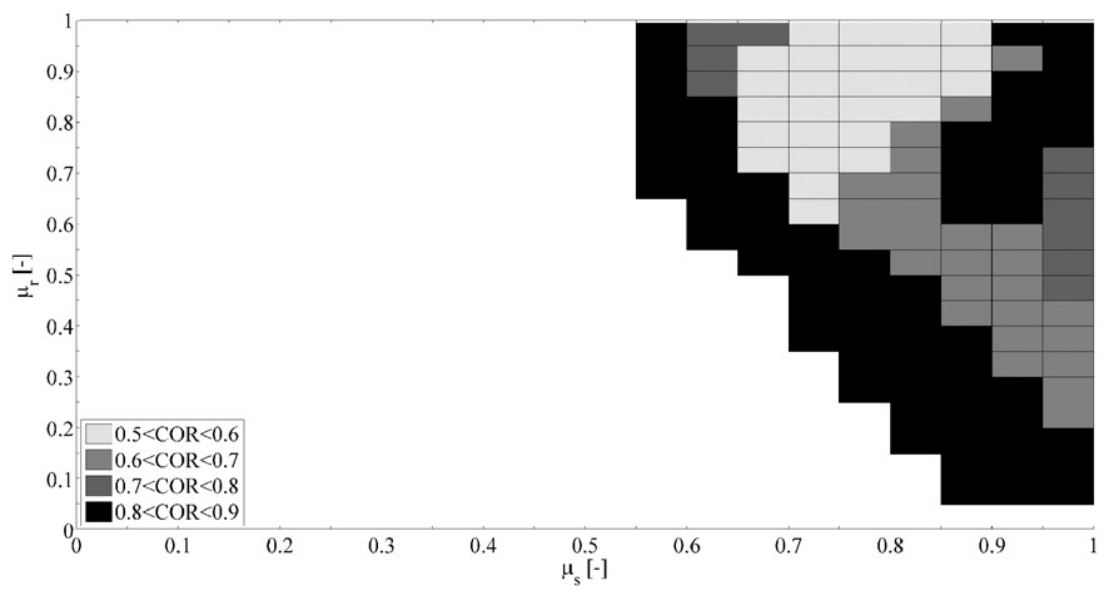

(b) Density plot, $S S C, \sigma_{n}=10,070 \mathrm{~Pa}, \mathrm{P}=1.0$
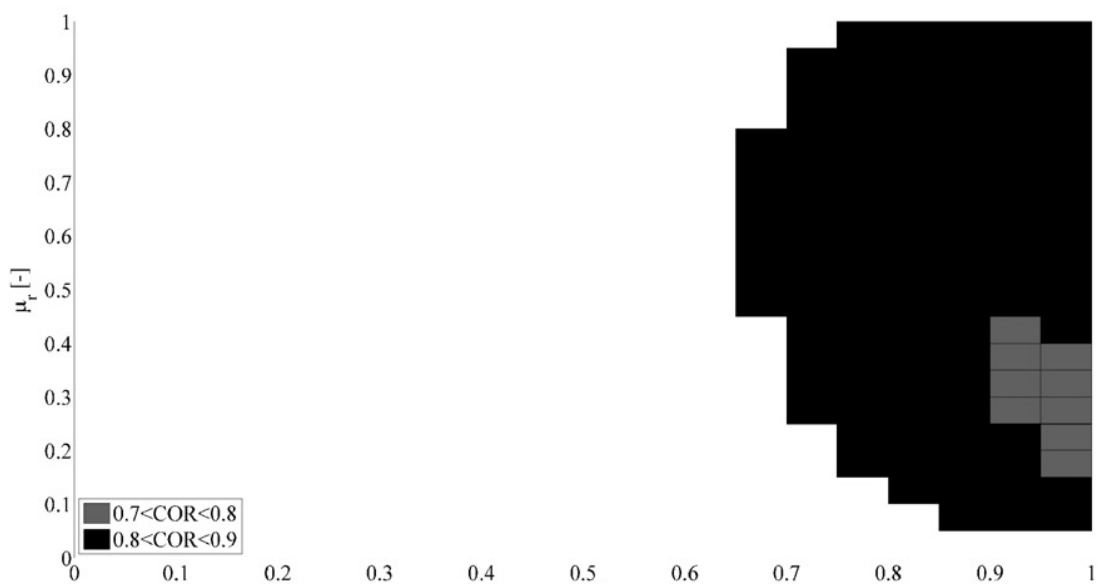

(c) Density plot, $S S C, \sigma_{n}=10,070 \mathrm{~Pa}, \mathrm{P}=1.2$

Fig. 8. Density plot comparison of shear cell tester (SSC) results. The marked combinations for $\sigma_{n}=10,070$ Pa are presented. Density plot of the particles' coefficient of restitution (COR) as a function of the coefficient of sliding friction $\left(\mu_{s}\right)$ and the coefficient of rolling friction $\left(\mu_{r}\right)$; in the white area, no valid sets of simulation parameters can be found. In each cell the valid sets are grouped according to the 4 different COR ranges. Each cell is coloured according to the group with the most members. The values plotted here were initially selected between the numerical values from the Artificial Neural Network with the original experimental results for the SSC, with a product coefficient $P=1.0$ (b). Subsequently, they were chosen with a lower virtual shear stress $(P=0.8)$ (a). The last image (c) represents the selection with a higher virtual shear stress $(P=1.2)$. 


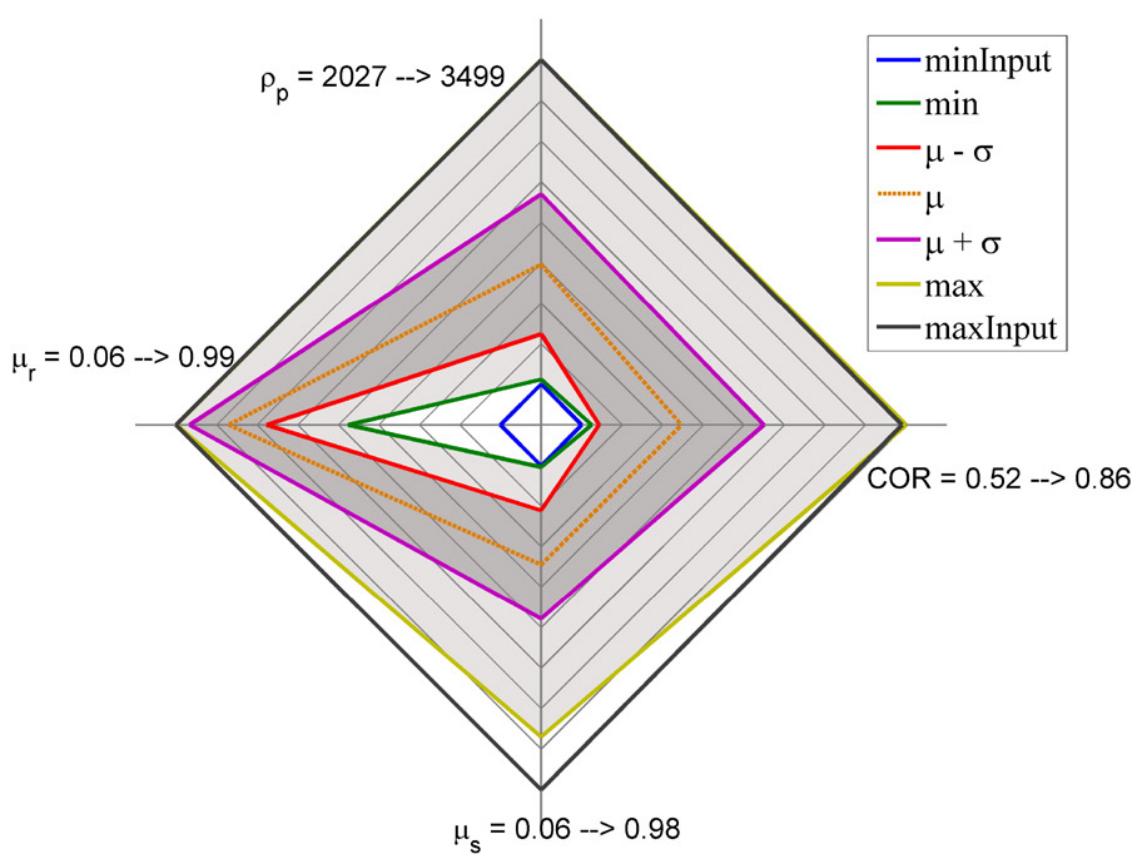

(a) Parameter space plot, $A o R_{\text {exp }}=38.85^{\circ}$

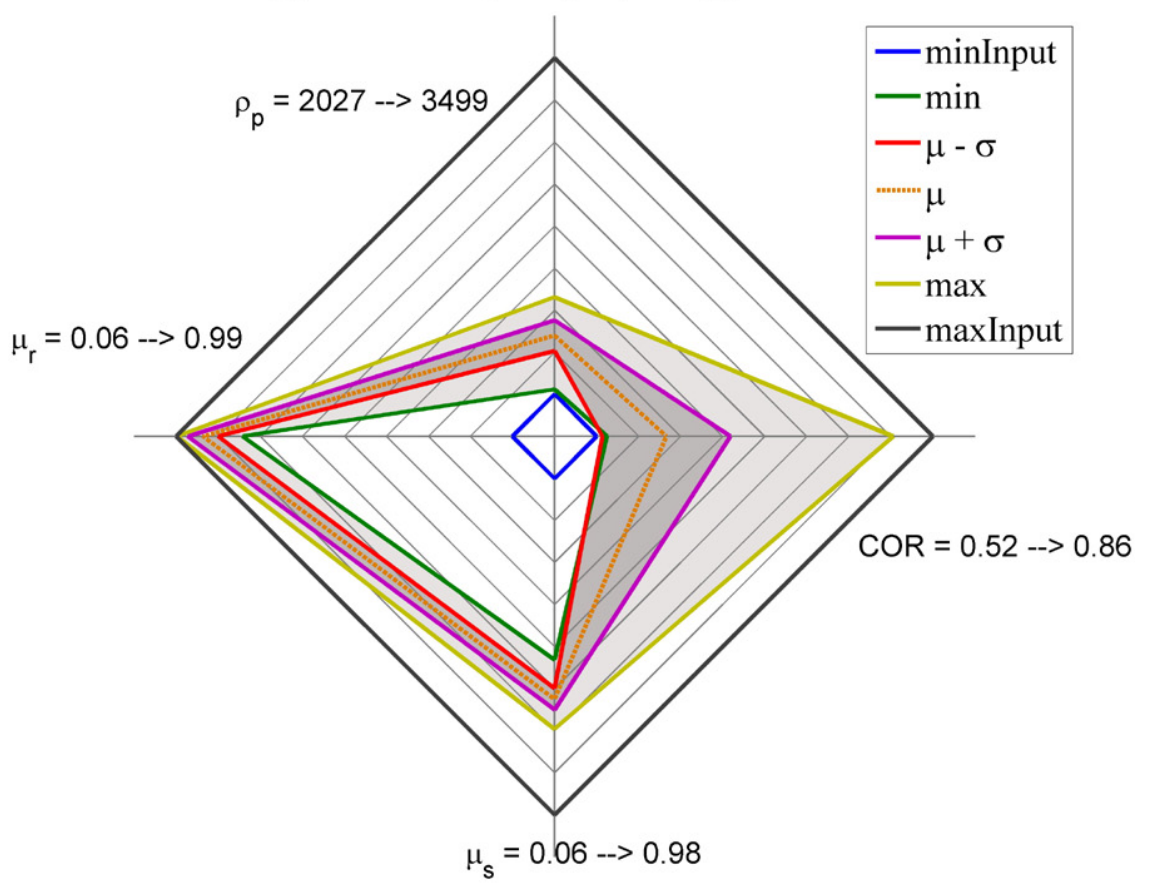

(b) Parameter space plot, $A o R_{\exp }=38.85^{\circ} \& S S C$ : $\sigma_{n}=10,070 \mathrm{~Pa}$

Fig. 9. Parameter space plots of valid simulation parameters for the angle of repose tester (AoR) and the combination of AoR and shear cell tester (SSC). Each axis of the parameter space plot represents one simulation parameter. The shaded area and dark shaded values indicate valid parameters combinations and the confidence interval, respectively. Further explanations are given in Section 3.3 .

identifications do not lead to a common set of valid parameters, most probably the chosen contact law was not applicable.

\section{Conclusions}

We have presented a two-step method for DEM simulation parameter identification. In the first step, an artificial neural network is trained using dedicated DEM simulations in order to predict bulk behaviours as function of a set of DEM simulation parameters of a given contact law. In the second step, this artificial neural network is then used to predict the bulk behaviour of a huge number of additional DEM parameter sets of the same contact law. The main findings of this study can be summarized as follows:

- An artificial neural network can be trained by a limited number of dedicated DEM simulations. The trained artificial neural network is then able to predict granular bulk behaviour.

- This prediction of granular bulk behaviour is much more efficient than computationally expensive DEM simulations. Thus, the macroscopic output associated with a huge number of parameter sets can be studied. 
- If the predictions of the artificial neural network are compared to a bulk experiment, valid sets of DEM simulation parameters can be readily deduced for a specific granular material.

- This DEM parameter identification method can be applied to arbitrary bulk experiments over the same material. Combining two artificial neural networks which predict two different bulk behaviours leads to winnowing the set of valid DEM simulation parameters.

- In turn, an empty intersection between the networks would be a clear indication that the chosen contact law is not appropriate.

- Artificial neural networks can establish the complicated relationship between microscopic DEM parameters of a given contact law and macroscopic properties of granular materials with less DEM simulations than direct procedures.

As part of future work, we will develop this method further by considering different fractions of granular materials, which will lead to sizedependent sets of DEM simulation parameters.

\section{Acknowledgements}

This study was funded by the K1-MET Metallurgical Competence Center in Linz and Leoben, Austria. The authors gratefully acknowledge its support.

\section{References}

[1] P.W. Cleary, M.L. Sawley, DEM modelling of industrial granular flows: 3d case studies and the effect of particle shape on hopper discharge, Appl. Math. Model. 26 (2) (2002) 89-111.

[2] P.A. Cundall, O.D.L. Strack, A discrete numerical model for granular assemblies, Geotechnique 29 (Issue 1) (1979) (47-65(18). URL http://www.icevirtuallibrary. com/content/article/10.1680/geot.1979.29.1.47).

[3] L. Vu-Quoc, X. Zhang, An accurate and efficient tangential force-displacement model for elastic frictional contact in particle-flow simulations, Mech. Mater. 31 (4) (1999) 235-269.

[4] A.D. Renzo, F.P.D. Maio, Comparison of contactforce models for the simulation of collisions in dembased granular flow codes, Chem. Eng. Sci. 59 (3) (2004) 525-541.

[5] C.M. Wensrich, A. Katterfeld, Rolling friction as a technique for modelling particle shape in dem, Powder Technol. 217 (0) (2012) 409-417.

[6] C. Kloss, C. Goniva, A. Hager, S. Amberger, S. Pirker, Models, algorithms and validation for opensource dem and cfddem, Prog. Comput. Fluid Dyn., Int. J. 12 (2) (2012) 140-152, http://dx.doi.org/10.1504/PCFD.2012.047457.

[7] A. Aigner, S. Schneiderbauer, C. Kloss, S. Pirker, Determining the coefficient of friction by shear tester simulation, 3rd International Conference on Particle-based Methods 2013, pp. 335-342.

[8] D. Hohner, S. Wirtz, V. Scherer, A numerical study on the influence of particle shape on hopper discharge within the polyhedral and multi-sphere discrete element method, Powder Technol. 226 (0) (2012) 16-28.

[9] J. Ai, J.-F. Chen, J.M. Rotter, J.Y. Ooi, Assessment of rolling resistance models in discrete element simulations, Powder Technol. 206 (3) (2011) 269-282.

[10] M. Combarros, H.J. Feise, H. Zetzener, A. Kwade, Segregation of particulate solids: experiments and dem simulations, Particuology 12 (0) (2014) 25-32.

[11] A. Alenzi, M. Marinack, C.F. Higgs, J.J. McCarthy, DEM validation using an annular shear cell, Powder Technol. 248 (0) (2013) 131-142

[12] M. Paulick, M. Morgeneyer, A. Kwade, Review on the influence of elastic particle properties on dem simulation results, Powder Technol. 283 (0) (2015) 66-76.
[13] S. Lommen, D. Schott, G. Lodewijks, DEM speedup: stiffness effects on behavior of bulk material, Particuology 12 (0) (2014) 107-112.

[14] S. Luding, Introduction to discrete element methods, basics of contact force models and how to perform the micromacro transition to continuum theory, EJECE 12 (2008) 785-826.

[15] V. Vidyapati, Constitutive modeling of dense granular flow based on discrete element method simulations. , Iowa State University, 2012 (Ph.D. thesis).

[16] B. Vaferi, F. Samimi, E. Pakgohar, D. Mowla, Artificial neural network approach for prediction of thermal behavior of nanofluids flowing through circular tubes, Powder Technol. 267 (0) (2014) 1-10.

[17] I. Witten, E. Frank, M. Hall, Data mining: practical machine learning tools and techniques, Morgan Kaufmann, 2005.

[18] S. Haykin, Neural networks and learning machines, no. v. 10, Prentice Hall, 2009 (2008034079. URL http://books.google.com.pk/books?id=K7P36lKzI_QC).

[19] Y.-H. Yang, Fundamental study of pore formation in iron ore sinter and pellets(Ph.D. thesis) University of Wollongong, 1990.

[20] B. Harthong, J.F. Jérier, P. Dorémus, D. Imbault, F.V. Donzé, Modeling of high-density compaction of granular materials by the discrete element method, Int. J. Solids Struct. 46 (18-19) (2009) 3357-3364.

[21] V. Baranau, D. Hlushkou, S. Khirevich, U. Tallarek, Pore-size entropy of random hardsphere packings, 2013.

[22] V. Baranau, U. Tallarek, Random-close packing limits for monodisperse and polydisperse hard spheres, 2014.

[23] L. Benvenuti, A. Aigner, D. Queteschiner, M. Combarros, S. Pirker, C. Kloss, Establishing the predictive capabilities of dem simulations: sliding and rolling friction coefficients of non-spherical particles, CFD 2014 Proceedings, 2014.

[24] N. Tsafnat, N. Amanat, A.S. Jones, Analysis of coke under compressive loading: a combined approach using microcomputed tomography, finite element analysis, and empirical models of porous structures, Fuel 90 (1) (2011) 384-388.

[25] J. Kovacik, Correlation between young modulus and porosity in porous materials, J. Mater. Sci. 18 (1999) 1007-1010.

[26] M.J. Jiang, H.S. Yu, D. Harris, A novel discrete model for granular material incorporating rolling resistance, Comput. Geotech. 32 (5) (2005) 340-357.

[27] D. Schulze, Powders and Bulk Solids: Behavior, Characterization, Storage and Flow, Springer, 2008.

[28] S.J. Antony, C.H. Zhou, X. Wang, An integrated mechanistic-neural network modelling for granular systems, Appl. Math. Model. 30 (1) (2006) 116-128.

[29] A.-K. Seghouane, Y. Moudden, G. Fleury, Regularizing the effect of input noise injection in feedforward neural networks training, Neural Comput. \& Applic. 13 (3) (2004) 248-254, http://dx.doi.org/10.1007/s00521-004-0411-6 (j2: Neural Comput and Applic.).

[30] D. Barrasso, A. Tamrakar, R. Ramachandran, A reduced order pbm-ann model of a multi-scale pbm-dem description of a wet granulation process, Chem. Eng. Sci 0 (2014).

[31] T. Varol, A. Canakci, S. Ozsahin, Artificial neural network modeling to effect of reinforcement properties on the physical and mechanical properties of al2024-b4c composites produced by powder metallurgy, Compos. Part B 54 (0) (2013) 224-233.

[32] A. Canakci, S. Ozsahin, T. Varol, Modeling the influence of a process control agent on the properties of metal matrix composite powders using artificial neural networks, Powder Technol. 228 (0) (2012) 26-35.

[33] M. Lashkarbolooki, B. Vaferi, M.R. Rahimpour, Comparison the capability of artificial neural network (ann) and eos for prediction of solid solubilities in supercritical carbon dioxide, Fluid Phase Equilib. 308 (1-2) (2011) 35-43.

[34] B. Das, U.P. Ganguly, N. Bar, S.K. Das, Holdup prediction in inverse fluidization using non newtonian pseudoplastic liquids: empirical correlation and ann modeling, Powder Technol. 273 (0) (2015) 83-90.

[35] S.M. Turp, B. Eren, A. Ates, Prediction of adsorption efficiency for the removal of nickel(ii) ions by zeolite using artificial neural network(ann) approach, Fresenius Environ. Bull. 20 (12) (2011) 3158-3165.

[36] W.L. Oberkampf, C.J. Roy, Verification and validation in scientific computing, Cambridge University Press, 2010 (URL http://books.google.com.pk/books?id= 7d26zLEJ1FUC). 


\section{Bibliography}

[1] Jun Ai, Jian-Fei Chen, J. Michael Rotter, and Jin Y. Ooi. Assessment of rolling resistance models in discrete element simulations. Powder Technology, 206(3):269-282, 1/30 2011.

[2] Andreas Aigner, Simon Schneiderbauer, Christoph Kloss, and Stefan Pirker. Comparison of simulation and experimental results of a simplified jenike shear tester. 6th International Conference on Discrete Element Methods, 20132013.

[3] Andreas Aigner, Simon Schneiderbauer, Christoph Kloss, and Stefan Pirker. Determining the coefficient of friction by shear tester simulation. 3rd International Conference on Particle-Based Methods, pages 335-342, 2013.

[4] A. Alenzi, M. Marinack, C. F. Higgs, and J. J. McCarthy. Dem validation using an annular shear cell. Powder Technology, 248(0):131-142, 112013.

[5] S. J. Antony, C. H. Zhou, and X. Wang. An integrated mechanistic-neural network modelling for granular systems. Applied Mathematical Modelling, 30(1):116-128, 12006.

[6] D. Aole, M. K. Jain, and M. Bruhis. New characterization methods for powder die fill process for producing powder metallurgical components. Powder Technology, 232(0):7-17, 122012.

[7] International ASTM. Astm d6128 - 06 - standard test method for shear testing of bulk solids using the jenike shear cell, 2013.

[8] International ASTM. Astm d6773 - 02 - standard shear test method for bulk solids using the schulze ring shear tester, 2013.

[9] Vasili Baranau and Ulrich Tallarek. Random-close packing limits for monodisperse and polydisperse hard spheres.

[10] Vasili Baranau, Dzmitry Hlushkou, Siarhei Khirevich, and Ulrich Tallarek. Pore-size entropy of random hard-sphere packings.

[11] Reza Barati, Seyed Ali Akbar Salehi Neyshabouri, and Goodarz Ahmadi. Development of empirical models with high accuracy for estimation of drag coefficient of flow around a smooth sphere: An evolutionary approach. Powder Technology, 257(0):11-19, 52014.

[12] Dana Barrasso, Ashutosh Tamrakar, and Rohit Ramachandran. A reduced order pbm-ann model of a multi-scale pbm-dem description of a wet granulation process. Chemical Engineering Science, $(0)$.

[13] Gabriel K. P. Barrios, Rodrigo M. de Carvalho, Arno Kwade, and Luis Marcelo Tavares. Contact parameter estimation for dem simulation of iron ore pellet handling. Powder Technology, 248(0): 84-93, 112013.

[14] Jacob Bear and Alexander Cheng. Modeling Groundwater Flow and Contaminant Transport. Springer, new york edition. 2010. 
[15] Niranjana Behera, Vijay K. Agarwal, Mark G. Jones, and Kenneth C. Williams. Modeling and analysis of dilute phase pneumatic conveying of fine particles. Powder Technology, 249(0):196-204, 112013.

[16] Yoav Benjamini. Opening the box of a boxplot. The American Statistician, 42(4):257-262, 1988. URL http://www.jstor.org/stable/2685133

[17] L. Benvenuti, C. Kloss, and S. Pirker. Identification of dem simulation parameters by artificial neural networks and bulk experiments. Powder Technology, 2016.

[18] Luca Benvenuti, Andreas Aigner, Daniel Queteschiner, M. Combarros, Stefan Pirker, and Christoph Kloss. Establishing the predictive capabilities of dem simulations: sliding and rolling friction coefficients of non-spherical particles. In CFD 2014 Proceedings, June 2014.

[19] Sofiane Benyahia and Janine E. Galvin. Estimation of numerical errors related to some basic assumptions in discrete particle methods. Industrial Engineering Chemistry Research, 49(21): 10588-10605, 11/03 2010. URL http://dx.doi.org/10.1021/ie100662z doi: 10.1021/ie100662z.

[20] R. Berger, C. Kloss, A. Kohlmeyer, and S. Pirker. Hybrid parallelization of the liggghts open-source dem code. Powder Technology, 278:234-247, 72015.

[21] R. J. Berry and M. S. A. Bradley. Investigation of the effect of test procedure factors on the failure loci and derived failure functions obtained from annular shear cells. Powder Technology, 174(1-2): 60-63, 5/16 2007.

[22] Tathagata Bhattacharya and J. J. McCarthy. Chute flow as a means of segregation characterization. Powder Technology, 256(0):126-139, 42014.

[23] Christopher M. Bishop. Pattern recognition and machine learning. springer, 2006.

[24] Anil Kumar Biswas. Principles of blast furnace ironmaking. Cootha Publishing House, xi 528, 23 $x 15 \mathrm{~cm}$, illustrated(, 1981, 1981 .

[25] Giuseppe Boccignone. Computazione per interazione naturale: Regressione lineare bayesiana.

[26] Howard E. Boyer and Timothy L. Gall. Metals handbook; desk edition. 1985.

[27] L. J. Briggs. Methods for measuring the coefficient of restitution and the spin of a ball. Journal of Research of the National Bureau of Standards, 34, 1945.

[28] Kay H. Brodersen, Christoph Mathys, Justin R. Chumbley, Jean Daunizeau, Cheng Soon Ong, Joachim M. Buhmann, and Klaas E. Stephan. Bayesian mixed-effects inference on classification performance in hierarchical data sets. The Journal of Machine Learning Research, 13(1):3133-3176, 2012.

[29] Chris Brown and Randal Nelson. Fitting experimental data, 2011.

[30] Petra Bubakova, Martin Pivokonsky, and Petr Filip. Effect of shear rate on aggregate size and structure in the process of aggregation and at steady state. Powder Technology, 235(0):540-549, 2 2013.

[31] Jeffrey W. Bullard and Edward J. Garboczi. Defining shape measures for 3d star-shaped particles: Sphericity, roundness, and dimensions. Powder Technology, 249(0):241-252, 112013.

[32] Aykut Canakci, Sukru Ozsahin, and Temel Varol. Modeling the influence of a process control agent on the properties of metal matrix composite powders using artificial neural networks. Powder Technology, 228(0):26-35, 92012. 
[33] John W. Carson and Harald Wilms. Development of an international standard for shear testing. Powder Technology, 167(1):1-9, 9/6 2006.

[34] A. Lumin Chen, B. Zhen Chen, and C. Ansheng Feng. Image analysis algorithm and verification for on-line molecular sieve size and shape inspection. Advanced Powder Technology, (0).

[35] Sebastian Chialvo, Jin Sun, and Sankaran Sundaresan. Bridging the rheology of granular flows in three regimes. Phys.Rev.E, 85(2):021305, Feb 2012. URL http://link.aps.org/doi/10.1103/ PhysRevE.85.021305

[36] Arthur Christopoulos and Michael J. Lew. Beyond eyeballing: fitting models to experimental data. Critical reviews in biochemistry and molecular biology, 35(5):359-391, 2000.

[37] Paul W. Cleary and Mark L. Sawley. Dem modelling of industrial granular flows: 3d case studies and the effect of particle shape on hopper discharge. Applied Mathematical Modelling, 26(2):89-111, 22002 .

[38] Paul W. Cleary, Rob Morrisson, and Steve Morrell. Comparison of dem and experiment for a scale model sag mill. International Journal of Mineral Processing, 68(1-4):129-165, 12003.

[39] C. J. Coetzee and R. G. Nel. Calibration of discrete element properties and the modelling of packed rock beds. Powder Technology, 264(0):332-342, 92014.

[40] M. Combarros, H. J. Feise, H. Zetzener, and A. Kwade. Segregation of particulate solids: Experiments and dem simulations. Particuology, 12(0):25-32, 22014.

[41] Kevin D. Costa, Steven Kleinstein, and Uri Hershberg. Model fitting and error estimation, 2010.

[42] P. A. Cundall and O. D. L. Strack. A discrete numerical model for granular assemblies. Geotechnique, 29(Volume 29, Issue 1):47-65(18), 1979. URL http://www.icevirtuallibrary.com/ content/article/10.1680/geot.1979.29.1.47

[43] Bimal Das, Uma Prasad Ganguly, Nirjhar Bar, and Sudip Kumar Das. Holdup prediction in inverse fluidization using non newtonian pseudoplastic liquids: Empirical correlation and ann modeling. Powder Technology, 273(0):83-90, 32015.

[44] Niklas Engblom, Henrik Saxen, Ron Zevenhoven, Henrik Nylander, and Gisle G. Enstad. Segregation of powder mixtures at filling and complete discharge of silos. Powder Technology, 215-216(0): 104-116, 12012.

[45] Zhi-Gang Feng and Samuel Gem Musong. Direct numerical simulation of heat and mass transfer of spheres in a fluidized bed. Powder Technology, 262(0):62-70, 82014.

[46] P. Frankowski, M. Paulick, M. Combarros, T. A. H. Simons, Arno Kwade, M. Schilling, H. J. Feise, and M. Morgeneyer. Material characterisation for discrete element modelling calibration. III International Conference on Particle-based Methods - Fundamentals and Applications PARTICLES 2013, 2013.

[47] V. Ganesan, K. A. Rosentrater, and K. Muthukumarappan. Flowability and handling characteristics of bulk solids and powders, a review with implications for ddgs. Biosystems Engineering, 101(4): 425-435, 122008.

[48] Maarten Geerdes, Renard Chaigneau, and Ivan Kurunov. Modern Blast Furnace Ironmaking: An Introduction (2015). Ios Press, 2015.

[49] D. Geldart, E. C. Abdullah, and A. Verlinden. Characterisation of dry powders. Powder Technology, 190(1-2):70-74, 3/5 2009. 
[50] LaTosha M. Gibson, Balaji Gopalan, Sarma V. Pisupati, and Lawrence J. Shadle. Image analysis measurements of particle coefficient of restitution for coal gasification applications. Powder Technology, 247(0):30-43, 102013.

[51] Christoph Goniva, Christoph Kloss, Niels G. Deen, Johannes A. M. Kuipers, and Stefan Pirker. Influence of rolling friction on single spout fluidized bed simulation. Particuology, 10(5):582-591, 102012 .

[52] Yan Grasselli and Hans J. Herrmann. On the angles of dry granular heaps. Physica A: Statistical Mechanics and its Applications, 246(3-4):301-312, 12/1 1997.

[53] Yan Grasselli, Hans J. Herrmann, Gadi Oron, and Stefano Zapperi. Effect of impact energy on the shape of granular heaps. 2(2):97-100, 2000. URL http://dx.doi.org/10.1007/s100350050039 journal: Granular Matter.

[54] Pierre A. Gremaud, John V. Matthews, and Meghan O'Malley. On the computation of steady hopper flows: Ii: von mises materials in various geometries. Journal of Computational Physics, 200 (2):639-653, 11/1 2004 .

[55] Pierre A. Gremaud, John V. Matthews, and David G. Schaeffer. On the computation of steady hopper flows iii: Model comparisons. Journal of Computational Physics, 219(1):443-454, 11/20 2006.

[56] A. P. Grima and P. W. Wypych. Investigation into calibration of discrete element model parameters for scale-up and validation of particle-structure interactions under impact conditions. Powder Technology, 212(1):198-209, 9/15 2011.

[57] Richard F. Grossman and R. J. Del Vecchio. Chapter 22 : Design of Experiments, page 515. Handbook of Vinyl Formulating. Wiley - Interscience, 2008.

[58] Jie Guo, Alan W. Roberts, and Jan-Dirk Prigge. Experimental investigation of wall pressure and arching behavior under surcharge pressure in mass-flow hoppers. Powder Technology, 258(0):272284, 52014.

[59] V. K. Gupta and Shivani Sharma. Analysis of ball mill grinding operation using mill power specific kinetic parameters. Advanced Powder Technology, (0).

[60] K. Gurney. An Introduction to Neural Networks. Taylor and Francis, 2003. ISBN 9780203451519. URL http://books .google.com.pk/books?id=sn6oBHq8qQQC

[61] G. Gustafsson, H. A Haggblad, P. Jonsen, and P. Marklund. Determination of bulk properties and fracture data for iron ore pellets using instrumented confined compression experiments. Powder Technology, 241(0):19-27, 62013.

[62] Alice Hager. Cfddem on multiple scales, an extensive investigation of particlefluid interactions, 2014.

[63] Alice Hager. CFDDEM on Multiple Scales, An Extensive Investigation of ParticleFluid Interactions. Christian Doppler Laboratory for Particulate Flow Modelling, 2014.

[64] Kevin J. Hanley, Catherine O’Sullivan, Jorge C. Oliveira, Kevin Cronin, and Edmond P. Byrne. Application of taguchi methods to dem calibration of bonded agglomerates. Powder Technology, 210(3):230-240, 7/10 2011.

[65] B. Harthong, J. F Jer, P. Dors, D. Imbault, and F. V DonzModeling of high-density compaction of granular materials by the discrete element method. International Journal of Solids and Structures, 46(1819):3357-3364, 92009. 
[66] Johannes Hartl and Jin Y. Ooi. Numerical investigation of particle shape and particle friction on limiting bulk friction in direct shear tests and comparison with experiments. Powder Technology, 212(1):231-239, 9/15 2011.

[67] S. Haykin. Neural Networks and Learning Machines. Number v. 10. Prentice Hall, 2009. ISBN 9780131471399. URL http://books.google.com.pk/books?id=K7P361KzI_QC. 2008034079.

[68] Jon Hilden, Keith Bowman, Ken Morris, Steve Wang, Omar Sprockel, and Bryan Ennis. Note on the interpretation of powder shear test data. Powder Technology, 182(3):486-492, 3/10 2008.

[69] M. Hirota, K. Takenaka, K. Iimura, and M. Suzuki. Proposal of an approximation equation for the yield locus to evaluate powder properties. Advanced Powder Technology, 18(3):287-302, 2007.

[70] D. Hohner, S. Wirtz, and V. Scherer. A numerical study on the influence of particle shape on hopper discharge within the polyhedral and multi-sphere discrete element method. Powder Technology, 226 (0):16-28, 82012.

[71] Richard G. Holdich. Fundamentals of Particle Technology. Midland Information and Technology and Publishing, 2002.

[72] Etienne Horn. The calibration of material properties for use in discrete element models, 2012.

[73] S. Humby, U. Tuzun, and A. B. Yu. Prediction of hopper discharge rates of binary granular mixtures. Chemical Engineering Science, 53(3):483-494, 21998.

[74] Kazuyoshi Iwashita and Masanobu Oda. Rolling resistance at contacts in simulation of shear band development by dem. Journal of Engineering Mechanics, 124(3):285-292, 1998.

[75] R. J. M. Janssen, M. J. Verwijs, and B. Scarlett. Measuring flow functions with the flexible wall biaxial tester. Powder Technology, 158(1-3):34-44, 10/27 2005.

[76] M. J. Jiang, H. S Yu, and D. Harris. A novel discrete model for granular material incorporating rolling resistance. Computers and Geotechnics, 32(5):340-357, 72005.

[77] Kerry Johanson. Effect of particle shape on unconfined yield strength. Powder Technology, 194(3): 246-251, 9/15 2009.

[78] Mohammad Khalilitehrani, Per J. Abrahamsson, and Anders Rasmuson. The rheology of dense granular flows in a disc impeller high shear granulator. Powder Technology, 249(0):309-315, 11 2013.

[79] A. H. Kharaz, D. A. Gorham, and A. D. Salman. Accurate measurement of particle impact parameters. Measurement Science and Technology, 10(1):31, 1999. URL http://stacks.iop.org/ $0957-0233 / 10 / i=1 / a=009$

[80] A. H. Kharaz, D. A. Gorham, and A. D. Salman. An experimental study of the elastic rebound of spheres. Powder Technology, 120(3):281-291, 10/22 2001.

[81] JuHyeon Kim and SangHwan Lee. Modeling drag force acting on the individual particles in low reynolds number flow. Powder Technology, 261(0):22-32, 72014.

[82] Christoph Kloss and Christoph Goniva. Liggghts manual. URL http://www.cfdem.com/

[83] Christoph Kloss, Christoph Goniva, Alice Hager, Stefan Amberger, and Stefan Pirker. Models, algorithms and validation for opensource dem and cfddem. Progress in Computational Fluid Dynamics, an International Journal, 12(2):140-152, 2012. URL http://dx.doi.org/10.1504/PCFD . 2012.047457 
[84] R. Kobylka and M. Molenda. Dem simulations of loads on obstruction attached to the wall of a model grain silo and of flow disturbance around the obstruction. Powder Technology, 256(0): 210-216, 42014.

[85] J. Kovacik. Correlation between young modulus and porosity in porous materials. Journal of Material Science, 18:1007-1010, 1999.

[86] Matthew Krantz, Hui Zhang, and Jesse Zhu. Characterization of powder flow: Static and dynamic testing. Powder Technology, 194(3):239-245, 9/15 2009.

[87] H. Kruggel-Emden, S. Wirtz, and V. Scherer. An analytical solution of different configurations of the linear viscoelastic normal and frictional-elastic tangential contact model. Chemical Engineering Science, 62(23):6914-6926, 122007.

[88] M. Kheiripour Langroudi, S. Turek, A. Ouazzi, and G. I. Tardos. An investigation of frictional and collisional powder flows using a unified constitutive equation. Powder Technology, 197(1-2):91-101, $1 / 102010$.

[89] M. Lashkarbolooki, B. Vaferi, and M. R. Rahimpour. Comparison the capability of artificial neural network (ann) and eos for prediction of solid solubilities in supercritical carbon dioxide. Fluid Phase Equilibria, 308(1-2):35-43, 9/25 2011.

[90] H. Li, G. R. McDowell, and I. S. Lowndes. A laboratory investigation and discrete element modeling of rock flow in a chute. Powder Technology, 229(0):199-205, 102012.

[91] Thomas Lichtenegger. Einfuhrung in dynamische partikelsimulationen. 2015.

[92] Stef Lommen, Dingena Schott, and Gabriel Lodewijks. Dem speedup: Stiffness effects on behavior of bulk material. Particuology, 12(0):107-112, 22014.

[93] Stefan Luding. Introduction to discrete element methods, basics of contact force models and how to perform the micromacro transition to continuum theory. EJECE, 12:785-826, 2008.

[94] Stefan Luding, Karsten Manetsberger, and Johannes Mllers. A discrete model for long time sintering. Journal of the Mechanics and Physics of Solids, 53(2):455-491, 2005.

[95] G. Lumay, F. Boschini, K. Traina, S. Bontempi, J. C. Remy, R. Cloots, and N. Vandewalle. Measuring the flowing properties of powders and grains. Powder Technology, 224(0):19-27, 72012.

[96] Tankeo M., Richard P., and Canot E. Analytical solution of the mu-i-rheology for fully developed granular flows in simple configurations. ArXiv e-prints, sep 2013. URL httpadsabs.harvard. eduabs2013arXiv1309.2267T. 1309.2267; Provided by the SAONASA Astrophysics Data System.

[97] CL Martin, LCR Schneider, L. Olmos, and D. Bouvard. Discrete element modeling of metallic powder sintering. Scripta Materialia, 55(5):425-428, 2006.

[98] Mohan Medhe, B. Pitchumani, and J. Tomas. Flow characterization of fine powders using material characteristic parameters. Advanced Powder Technology, 16(2):123-135, 2005.

[99] J. Mellmann, T. Hoffmann, and C. Furll. Flow properties of crushed grains as a function of the particle shape. Powder Technology, 249(0):269-273, 112013.

[100] Hiroshi Mio, Ko Yamamoto, Atsuko Shimosaka, Yoshiyuki Shirakawa, and Jusuke Hidaka. Modeling of solid particle flow in blast furnace considering actual operation by large-scale discrete element method. ISIJ International, 47(12):1745-1752, 2007. 
[101] Hiroshi Mio, Satoshi Komatsuki, Masatoshi Akashi, Atsuko Shimosaka, Yoshiyuki Shirakawa, Jusuke Hidaka, Masatomo Kadowaki, Shinroku Matsuzaki, and Kazuya Kunitomo. Validation of particle size segregation of sintered ore during flowing through laboratory-scale chute by discrete element method. ISIJ International, 48(12):1696-1703, 2008. journal: ISIJ International.

[102] B. K. Mishra and Raj K. Rajamani. The discrete element method for the simulation of ball mills. Applied Mathematical Modelling, 16(11):598-604, 111992.

[103] Paul A. Moysey, Nadella V. Rama Rao, and Malcolm H. I. Baird. Dynamic coefficient of friction and granular drag force in dense particle flows: Experiments and dem simulations. Powder Technology, 248(0):54-67, 112013.

[104] Patric Muller, Michael Heckel, Achim Sack, and Thorsten Poschel. Complex velocity dependence of the coefficient of restitution of a bouncing ball. Phys.Rev.Lett., 110(25):254301, Jun 2013. URL http://link.aps.org/doi/10.1103/PhysRevLett.110.254301.

[105] Shungo Natsui, Shigeru Ueda, Fan Zhengyun, Junya Kano, Ryo Inoue, and Tatsuro Ariyama. Sensitivity analysis of physical parameters in discrete element method compared with blast furnace cold model experiments. 2010.

[106] W. L. Oberkampf and C. J. Roy. Verification and Validation in Scientific Computing. Cambridge University Press, 2010. ISBN 9781139491761. URL http://books.google.com.pk/books?id= 7d26zLEJ1FUC

[107] M. Paulick, M. Morgeneyer, and A. Kwade. Review on the influence of elastic particle properties on dem simulation results. Powder Technology, 283(0):66-76, 102015.

[108] Gerald G. Pereira and Paul W. Cleary. Multi-component granular segregation in a rotary classifier. 2012.

[109] GG Pereira, S. Pucilowski, K. Liffman, PW Cleary, and Melbourne Vic. Streak patterns in binary granular media in a rotary classifier. 2009.

[110] GG Pereira, MD Sinnott, PW Cleary, Kurt Liffman, Guy Metcalfe, and Ilija D. utalo. Insights from simulations into mechanisms for density segregation of granular mixtures in rotating cylinders. Granular Matter, 13(1):53-74, 2011.

[111] GG Pereira, N. Tran, and PW Cleary. Segregation of combined size and density varying binary granular mixtures in a slowly rotating tumbler. Granular Matter, 16(5):711-732, 2014.

[112] S. J. Plimpton. Fast parallel algorithms for short-range molecular dynamics. J Comp Phys, 117: $1-19,1995$.

[113] Thorsten Poschel and Thomas Schwager. Computational Granular Dynamics. Springer, 2004.

[114] Carl Edward Rasmussen and Hannes Nickisch. Gaussian processes for machine learning (gpml) toolbox. The Journal of Machine Learning Research, 11:3011-3015, 2010.

[115] C J Reagle, J M Delimont, W F Ng, and V.P.Rajendran S V Ekkad and. Measuring the coefficient of restitution of high speed microparticle impacts using a ptv and cfd hybrid technique. Measurement Science and Technology, 24(10):105303, 2013. URL http://stacks.iop.org/0957-0233/24/i= $10 / \mathrm{a}=105303$

[116] Alberto Di Renzo and Francesco Paolo Di Maio. Comparison of contactforce models for the simulation of collisions in dembased granular flow codes. Chemical Engineering Science, 59(3):525-541, 22004. 
[117] Rudiger Schmitt and Hermann Feise. Influence of tester geometry, speed and procedure on the results from a ring shear tester. Particle and Particle Systems Characterization, 21(5):403-410, 2004.

[118] D. Schulze. Time- and velocity-dependent properties of powders effecting slip-stick oscillations. Chemical Engineering Technology, 26(10):1047-1051, 2003.

[119] Dietmar Schulze. Powders and bulk solids: behavior, characterization, storage and flow. Springer, 2008.

[120] Dietmar Schulze. Flow properties of powders and bulk solids, 2011.

[121] Dietmar Schulze, H. Heinrici, and H. Zetzener. The ring shear tester as a valuable tool for silo design and powder characterization. Powder Handling and Processing, 13(19-26), 2001.

[122] Jorg Schwedes. Measurement of flow properties of bulk solids. Powder Technology, 88(3):285-290, 91996.

[123] Jorg Schwedes. Review on testers for measuring flow properties of bulk solids. 5(1):1-43, 2003. URL http://dx.doi.org/10.1007/s10035-002-0124-4 journal: Granular Matter.

[124] A. Schweiger and I. Zimmermann. A new approach for the measurement of the tensile strength of powders. Powder Technology, 101(1):7-15, 1/4 1999.

[125] Abd-Krim Seghouane, Yassir Moudden, and Gilles Fleury. Regularizing the effect of input noise injection in feedforward neural networks training. Neural Computing and Applications, 13(3):248254, 09/01 2004. URL http://dx.doi.org/10.1007/s00521-004-0411-6. J2: Neural Comput and Applic.

[126] Erdem Simsek, Florian Sudbrock, Siegmar Wirtz, and Viktor Scherer. Influence of particle diameter and material properties on mixing of monodisperse spheres on a grate: Experiments and discrete element simulation. Powder Technology, 221(0):144-154, 52012.

[127] U. Sindel and I. Zimmermann. Measurement of interaction forces between individual powder particles using an atomic force microscope. Powder Technology, 117(3):247-254, 6/25 2001.

[128] Dale M. Snider. Three fundamental granular flow experiments and cpfd predictions. Powder Technology, 176(1):36-46, 7/10 2007.

[129] Jin Sun and Sankaran Sundaresan. Radial hopper flow prediction using a constitutive model with microstructure evolution. Powder Technology, 242(0):81-85, 72013.

[130] K. Takenaka, K. Iimura, M. Suzuki, and M. Hirotab. Shape effects of the yield locus on the rankine coefficient. Advanced Powder Technology, 19(1):25-37, 2008.

[131] Gabriel I. Tardos. A fluid mechanistic approach to slow, frictional flow of powders. Powder Technology, 92(1):61-74, 61997.

[132] Jose' M. Torralba, Luz Fuentes-Pacheco, Nerea Garcia-Rodriguez, and Monica Campos. Development of high performance powder metallurgy steels by high-energy milling. Advanced Powder Technology, 24(5):813-817, 92013.

[133] Naomi Tsafnat, Negin Amanat, and Allan S. Jones. Analysis of coke under compressive loading: A combined approach using microcomputed tomography, finite element analysis, and empirical models of porous structures. Fuel, 90(1):384-388, 12011.

[134] Sinan M. Turp, Beytullah Eren, and Asude Ates. Prediction of adsorption efficiency for the removal of nickel(ii) ions by zeolite using artificial neural network(ann) approach. Fresenius Environmental Bulletin, 20(12):3158-3165, 2011. 
[135] B. Vaferi, F. Samimi, E. Pakgohar, and D. Mowla. Artificial neural network approach for prediction of thermal behavior of nanofluids flowing through circular tubes. Powder Technology, 267(0):1-10, 112014.

[136] Temel Varol, Aykut Canakci, and Sukru Ozsahin. Artificial neural network modeling to effect of reinforcement properties on the physical and mechanical properties of al2024-b4c composites produced by powder metallurgy. Composites Part B: Engineering, 54(0):224-233, 112013.

[137] Vidyapati Vidyapati. Constitutive modeling of dense granular flow based on discrete element method simulations, 2012.

[138] Loc Vu-Quoc and Xiang Zhang. An accurate and efficient tangential force-displacement model for elastic frictional contact in particle-flow simulations. Mechanics of Materials, 31(4):235-269, 4 1999.

[139] C. M. Wensrich and A. Katterfeld. Rolling friction as a technique for modelling particle shape in dem. Powder Technology, 217(0):409-417, 22012.

[140] Wikipedia. Discrete element method. URL http://en.wikipedia.org/wiki/Discrete_element_ method.

[141] Ian Witten, Eibe Frank, and Mark Hall. Data Mining: Practical machine learning tools and techniques. Morgan Kaufmann, 2005.

[142] M. Wojcik, J. Tejchman, and G. G. Enstad. Confined granular flow in silos with inserts - full-scale experiments. Powder Technology, 222(0):15-36, 52012.

[143] Santiago Ramon y Cajal. Histologie du syst nerveux de l'homme des vert. Maloine, 1911. URL https://books.google.at/books?id=fww4rgEACAAJ

[144] Yan-Hui Yang. Fundamental study of pore formation in iron ore sinter and pellets, 1990.

[145] Hideto Yoshida, Tetsuya Yamamoto, Yasushige Mori, Hiroaki Masuda, Kenji Okazaki, and Hirosuke Sugasawa. Particle size measurement of reference particle candidates and uncertainty region of count and mass based cumulative distribution. Advanced Powder Technology, (0).

[146] Y. C. Zhou, B. H. Xu, A. B. Yu, and P. Zulli. An experimental and numerical study of the angle of repose of coarse spheres. Powder Technology, 125(1):45-54, 5/13 2002. 


\section{Curriculum Vitae}

\section{Personal Data}

Name Dipl.-Ing. Luca Benvenuti

Email lucabenvenuti@gmail.com

Date of Birth May $12^{\text {th }}, 1988$

Citizenship Italy

Parents Fabrizio Benvenuti, Daniela Zanoni

\section{Professional Experience}

2013 - 2016 Researcher at the Department of Particulate Flow Modelling at Johannes Kepler University in Linz, Austria

Site Engineer and Quantity Surveyor, Ferretti International, LISCO (Libya).

\section{Education}

$2013-2016$

2012

2011

$2010-2012$

$2007-2010$

2007

$2002-2007$
PhD-Student of Mechatronics (Doktoratstudium) at Johannes

Kepler University in Linz, Austria

Diploma in Civil Engineering (Dipl.-Ing.) at the Politecnico of Milano, Italy

Erasmus in Civil Engineering at the Ecole Special de Travaux Publics of Paris, France.

Student of Civil Engineering (Master) at the Politecnico of Milano, Italy

Student of Civil Engineering (Bachelor) at the Politecnico of Milano, Italy

Matura at the Liceo Bagatta in Desenzano del Garda, Italy

Schulverein at the Liceo Bagatta in Desenzano del Garda, Italy 


\section{Eidesstattliche Erklärung}

Ich erkläre an Eides statt, dass ich die vorliegende Dissertation selbstständig und ohne fremde Hilfe verfasst, andere als die angegebenen Quellen und Hilfsmittel nicht benutzt bzw. die wörtlich oder sinngemäß entnommenen Stellen als solche kenntlich gemacht habe. Die vorliegende Dissertation ist mit dem elektronisch übermittelten Textdokument identisch.

Linz, 24. März 2016 


\section{Sworn Declaration}

I hereby declare under oath that the submitted doctoral dissertation has been written solely by me without any outside assistance, information other than provided sources or aids have not been used and those used have been fully documented. The dissertation here present is identical to the electronically transmitted text document.

Linz, 24. März 2016 